


$$
y^{\ln }
$$

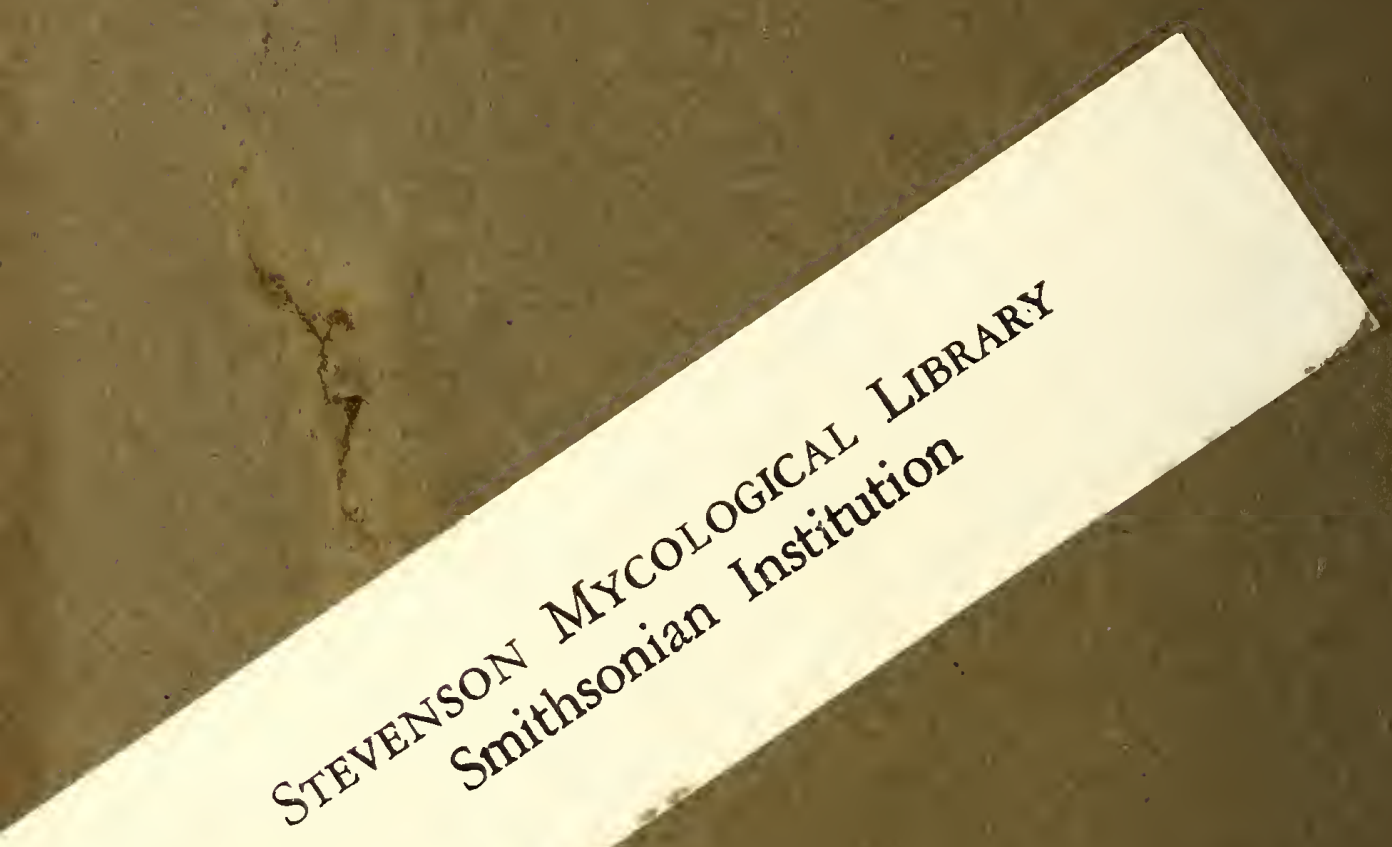








\section{LICHENOGR APHIAE}

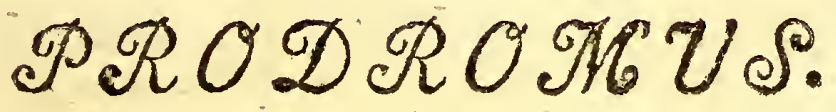

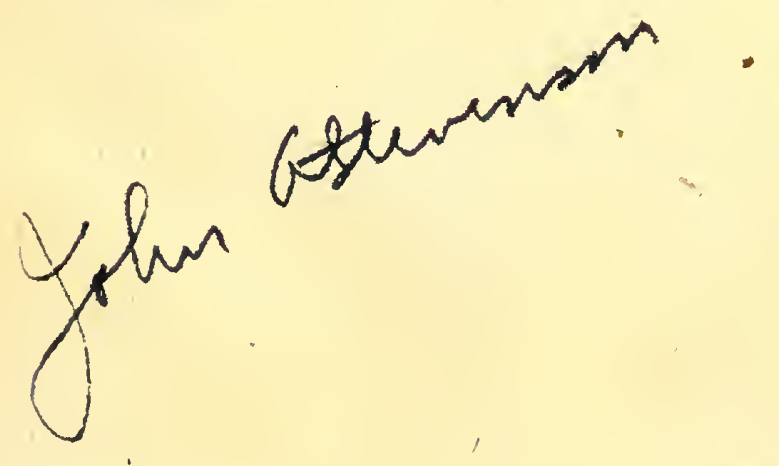


Wer hat je die Flechten, wer hat die Moose gezâhlet,

Deren Frühling beginnt, wen Fróste den Herbst entblattern,

Deren üppiger Wachs die Scheitel âtherischer Alpen

$D a$, wo sie Flora verlåst, mit tausend Farben bekleidet?

J. G. HERDER Briefe zu beförd. der Humanität. 
Lav: Dalton Mob: Pienson
Amicus amies.

\section{LICHENOGRAPHIAE,}

\section{SVECICAE}

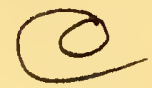

\section{PRODROMUS.}

\section{AUctor}

\section{Erik Acharius}

Med. Duct. Medicus Provinc. Ostro-Gothice ad Nosocom.Reg. Vadusten. Med. Ordin.

Reg.Acad. Scient. Svec. 是 Societ. Physiogr.Luind. Membr.
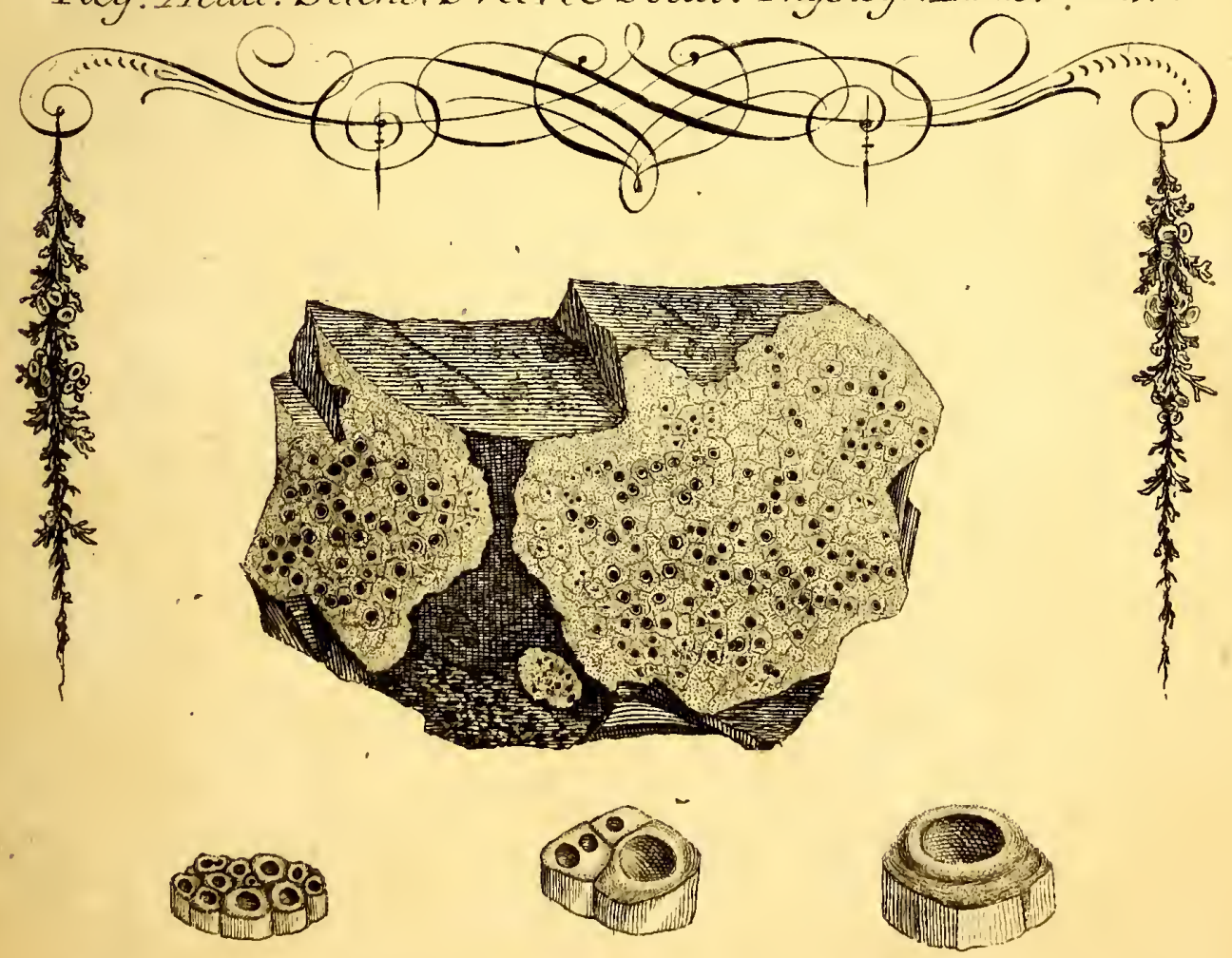

Lincopiae, D.G.Bjöm, $179^{8}$ — 

INCLIT 2

\section{QUAE LONDINI FLORET}

SOGJET ATJ LYFT T THAN AT

\section{SACRUM VOLUIT}

AUCTOR. 



\section{PROOEMIUM.}

pufculum pluribus numeris abfolutum, Lichenes complegtens' fvecanos hucusque detectos, prelo fubjicere in animum induxi. Verum enim vero tanta cum fic fpecierum copia, quas inter plures novæ, immo \& multæ minus recte antea cognitie mea cura delineari, arique incidi ac pingi debuisfent, defcriptionibus ac obfervationibus unicuique earum accurate fubjundis; hic certe conatus in multum temporis produceretur, præfertim quum delata mihi munera longe aliam expofcunt operam meque idemtidem ab hifce meis revocent deliciis; malui igitur, quam neetere moras, antecapere diem proindeque hoc Spicilegium meo qualicunque ftudio elucubratum, Prodromi inftar, præmittere.

Hanc vero meam operam Hiftoriæ naturalis cultoribus haud inutilem fore putavi, quippe illis probe notum eft, quamquam haud pauca hoc tempore de Lichenibus eo- 
rumque permultis nuvis tpeciebus defcripta habemus, defiderari tamen necesfariam de hus cretitudinem, utpote quæ obfervationibus exacte fapiusque inftitutis, minime vero ab unoquoque individuo in mufeis, five fub excurionibus, bis aut femel forte invento, obcineri potent.

In hac nimirum indagatione plurium annorum fpatio occupatus credo equidem me, præfifcine dixerim, in fpecierum determinatione aliquanto plus efrecisfe, quam quod hucusque in Lichenum cognitione facrum effe novi. Neque tamen reticendum eft, hoc in negotio auxiliares mihi benignasque manus porrexisfe aliquot amicos \& populares neos xutumatisfimos. Hos inter srata, ut decet, mente honorisque causfa nomino Celeberrimos Viros SWARTZ \& WESTRNG, quorum llle, ingenti, qua pollet, harum rerum peritia, labores meos fublevavit atque opufculum fuis detedis pluribus in locis ditatum confcriptumque benevole perluftravit; Hic vero in multis a fe inventis \& ingeniola follertia analytice examinatis fpeciebus differentiam plumimarum evidentiorem reddidit, \& hinc in characteribus harum fpecierum determinandis planiorem certioremque mihi apperuit viam. Varios etiam novos \& illuftratos Lichenes fagacisfmi obfervatoris LJUNGH debeo indu- 
induftriæ ac perveftigationi prorfus fingulari: Nec parvi momenti funt ii, quos familiarisfime mecum communicarunt Celeberr. acerrimique almæ naturæ inveitigatores, THUNBERGIUM loquor, RETZIUM, AFZELIUM, alios ut taceam.

Quas in fcenam produxi, novas fpecies veluti certas habendas effe, quemlibet æquum non diffiteri credo æutimatorem, paucas fi forte exceperim. Harum partem jam dudum in Novis Actis Reg. Acad. Scient. Svec. his proxime præteritis annis defcripfi \& delineavi. Reliquas tam novas, quam $\mathrm{ab}$ Auctoribus antea commemoratas fat 0 mnino a me jam determinatas effe, Ifpero atque confido.

Imperata mihi brevitas haud finit"rationes adjungere, quibus dueus in quarundam fpecierum doctrina ab aliorum defcedere aufim fententia. Hæc aliaque huc pertinentia in uberiorem, fi fata concesferint, de hifce naturæ fotibus disfertationem refervanda putavi. lccirco iniquiori me cenfura ad id usque tempus vexaturum credo neminem. Verum hoc fi inopinato evenerit, tunc demum occafio mihi fuppetit idonea diffentientes tanto majori nifu convincendi, quanto latior mihi patefcit exfpatiandi campus. Correctiones vero \& emendationes, genai- 
no fubfruetas experimentorum obfervatio. numque fundamento, ingenue venerabor $\&$ ambabus amplectar manibus. Inmo easdem in meos allorumque ufus vertere haud inglorium ducam; quippe dies diem docet.

Scientia Natura incremento augetur perpetuo, \& temporis progreffu majus celte \& fape infperatum obontur lumen. Procul dubio intra fines Svecia permulta nova \& huc usque non fatis examinatre Lichenum fpecies inveniuntur, prafertim quonian plura longe dislica \& nondum vifitata Inca refant, in hac fuo jure pre aliis nominanda Lichenum patria. Repetitis obfervationibus, a nofris pariter atque exteris Natura fitudiofis follicice inftitutis, eveniet profeero, ut major in dies fpecierum hoc in Prodromo allatarum adquiratur cognitio. Plures quoque apud varios AuEtores forte obvenient fpecies, a me ideo præterita, quod accurate minus defcriptæ haud in cenium deferri potuere. Fortasfis etiam aliquæ videbuntur fuo loco haud rite dispofitæ, aliæ autem ea propter penitus intermisfæ, quod nonnullorum hujus argumenti fcripta mihimet haud incotuere. Itaque conabor accuratius colligere \& hifce meis ftudiis amplius addere quidquid ad ulteriorem illufrationem, emendationem \& perfectionem conducere queat. Ifthoc nimirum,

tam- 
tamquam Supplementum Libelli, pofthac in lucem edere, \& fic quidem rependere, quod in præfentia fortasfis defideretur, mihi confitutum eft.

Sxpius exteri Scriptores minus rite cognoverunt fpecies a LINN $\mathbb{E}$ Noftro jam pridem commemoratas, ac proinde eis romina tribuerunt non numquam peregrina \& prorfus impropria. Confufionem inde exortam, certiori nixus, rei notitia, quantum in me fuit, tollere ftudui. Hinc equidem fpero me paullo accuratius explanasfe fpecies ab Illuftri Viro allatas.

His ceterisque fpeciebus fvecanis ejusmodi differentias fpecificas five characteres adfignavi, quibus ipfex \& his connexæ varietates luculentius \& tutius dignofcantur. Hæc vero tanto magis necesfaria fuit opera, quanto certius eft, nonnullos eamdem plus júfto neglexisfe. Mirum igitur non eft, glifcere fape errores in dignofcenda, quam defignatam voluere, fpecie, quum haud raro iidem, vagi \& incerti charaeteres, pluribusque communes, apud ipfos obveniant. Quin etiam hinc evenit, ut in familia, cui unaquæque fpecies adnumere. tur, adfignanda fæpenumero hallucinemur. Infuper cuivis fcripta eorum æqua penfitanti lance in confesfo eft, hos nimium quan. 
tum in id propenfos fuisfe, ut novas multas. que effingant fpecies, ubi vel minima harum differentia aut varietas fefe offert. Itaque operæ pretium judicavi ftudium qualecumque meum in id impendere, ut aquam reformationem, natura ipfa juftisque ac follicite inftitutis obfervationibus innixam, quo. ad ejus fieri posfit, hoc fufcipiam fpecimine.

Ob eamdem hanc causfam necesfarium omnino fuit, cuicumque antea cosnitæ fpeciei nomina ab Auctoribus ei impolita, ut fynonyma, addere, immo etiam omnes adjungere $a b$ eis nominatas \& quafi diftindas fpecies receptas, licet hæ veluti varietates tantummodo habendæ int. Hinc nimirum perturbatio fpecierum \& varietatum, ab accurata minus indagatione profecta, cohibeatur, \& in pofterum evanefcat oportet.

Optimas \& fere fingulas, quas habemus, Licherum icones fedulo adnotavi; præcipuas eorum varietates \& habitationis loca fimul exponens. Obfervatiunculas, brevitatis fudiofus, quancum fieri potuit, quibusdam addidi fpeciebus. Nomina etiam fvecana, licet pasfim a me ficta, haud excludenda putavi.

Quum præterea in genere adeo multiplici minus adivefacto, difficile in primis foret 
foret eas, quas quærit, reperire fpecies, nimirum fecundum fubdivifiones Methodo Linnæana receptas, haud abs re vifum eft iftam apponere Dianomen, quantumvis non nihil commutatam, quam jam antea in Nov. At. Reg. Acad. Sc. Svec. T. XV. p. 244. feqq. exhibui. Id vero eo magis mihi curæ fuit, quo certius, finem methodi artificialis, apprime utilis ac necesfariæ, fecundum fundamenta heic rite obfervanda, me adfecutum esfe crediderim. Scilicet Naturæ indicia Lichenumque habitum externum, quippe quo facilior perquirendi opera exiftit, obfervare \&t confulere omnino neceffum eft. Tantum vero abeft, ut aliorum hoc in negotio tentamina rejicienda putem, ut id potius, cuius demum Auctoris fit, in ufum convertere ftuduerim, quod intento fini convenientisfmum \& fcientiæ maxime profuturum cenfui.

Ad Lichenum Tribus hoc in cenfu no. minatas, quod adtinet, quivis, fi placet, \& validior fubeft ratio, totidem fingat genera. Plurimas tamen ob causfas, easque haud fpernendas 111. LINNEUS non nifi unicum ftatuit Genus a). Recentioris ævi percele-

a) Charabter hujus Generis in Gener. Plane. LINNEEl Edit. Schreb. datus, Veficslis, farine 
celebres Botanici eumdem hunc fecuti funt magni nominis antefignanum. Me certe hucusque nec propria indagatio, nec aliorum demonftrationes impulere, quin devenerandi olim PRECEPTORIS mei fententiam, absque omni partium fudio, velut recisfimam fequar $b$ ). Antequam hac in re aliquod cum fuccesfu negorium furcipiatur, me quidem judice, multo perfectior Liche-

num

initar tenuisfimæ, ad fuperficiem fparfis \& congeftis, (Pollinis 1. genitura mafculinæ ana. logis?) atque Receptacislo lubtrotundo, concavo, plano, convexo, lenina fic dicta in feries digefta continente, (Yericarpio plantarum, feu potius Recentaculo frućtíficationis vel fru. Ctus refpondente?) depromtus, inter reliquos forte eminet. Certa quiden ratione conjecturalem esfe, nullus facile negabit, fed exiam obfervationibus conlentaneum is judicabit, cui detecta $\mathrm{Cl}$. HEDWIGII nota funt.

4) "Attamen cum hæ partes, flores nempe \& fe"mina, extra vilum ut cum DILLENIO lo"quar, plerumque locentur, vel faltem fint "ejusmodi, ut nullæ inde vel tamen difficilio"res, ex quibus ob nimiam partium diftin"guendarum parvitatem multum levaminis in de. "terminandis generibus vel fpeciebus exfpecta"veris, notæ characterifticæ defumi queant, luf"ficit nobis crasior confideratio tiguræ, habi"tualis nempe, qua ab aliis Generibus \& inter "fe facile Lichenes diftinguuntur." Hoffm. Comment, de vario Lichensem UJu. p. 14. 
num fecundationis, organoramque prop?gationis ac fortasfis fruetificationis \& disfeminationis anteponenda eft cognitio. $Q$ a ר. to enim Lichenes a Plantis conformatione, c) indole atque natura fua fingulari receduns, tanto difficilior noftra exiftic opera, in in plura genera eos difpefceremus, nimingm quando horum Characteres ex iisdem Fruetificationis fundamentis defumere conare. mur. Quis vero organa Lichenum fexualia vidit \& cerce demonftravit? Quis my tiri. um fecundationis eorum detegere adhuc valuit

-) Radicatio Lichenum nulla, nec proprie fic diet $\mathrm{f}$ flia. tio, neque Plantarum confinilis organiatio. - Ve. cesfum erat, ut more Lithophytorum ac Znophyto um puncto quodam lapidi, terr $x$ vegeta. bilium partibus varis af ixi tenerentur Lichenes; fed neque hac via fucci eorum nutritii depro. muntur, neque ita funt comparata partes ille. quibus adhærelcunt, ut vices radicum fugentium implere queant. - In modum foliormin expli. cantur atque expanduntur quampluti ni Leh:nes, fed eodem ac in Planis officin folia ita fungi, nulla experientia probatun elf, nec fa. cile probari poteft.' Fabrica etiam diverisima. Quis Całum Tunam 1. O untian, ex f slio prolifero conftantem, autumet? Noine potius a phyllam praticaret Botanicus meliora edot licet folia fucculenta \& ejustem fere forme in aliis plantis ocurrere non ignoret. Ad esfenciarn folii figura nihil confert externa.

2. 
valuit? d) Sin exftierint hæ fruetificationis partes, haud tamen fufficientem earum ha. bemus notitiam, fine qua, fecundum jam jam rata atque confirmata fundamenta, diverfa ftatuere genera nec licet, nec oportet. e)

Quod

d) Quantuin ego quidem fcio nulla, vel plane decifa, gure lichenum anthelin explicarent atque proba. rent, adhuc cum fucceslu inftituta fuere experimen. ta.- Fulcherrime late funt, quas peritia fumma \& follertia, fibi omnino propria, inltituit obfervationes $\mathrm{Cl}$. HEDWIG, \& grave ingenique plenum elt Ejus de hilce Lichenum partibus judicium; dolendum tamen, quod paucas tantum lpecies examini fubjecerit, \& experimentorum necesfariorum luffragio deftituatur fagacislimi Viri Theoria. - Nique tamen MICHELII atque SCHM IDELII obfervationibus deroganda fides. Palam Hi teltantur fe pulverem iftud organicum (Semin MICHELII) in fuperficie Lichenum difperfum, germinantem vidisle fubo. lemque multiplicantem. LIN N $\mathbb{E} I$. olim fententia hoc asfertum etiain concordare, in aperto elt. Neque HOFFMANNUM de hac re difientientem reperio neque SCHREBERUM. (HOFF M. 1. c. p. I1 - 13.) Corpufcula illa Hedwigiana, didyma aut fphacica intra receptacula nidulantia, vel fi mavis lemina, quid fata ilthec produxerunt?

(5) Mex quidem fententize haud parum roboris conciliant noftri temporis inventa, ad Mufcos nempe quod attinet. Scilicet ante commixi \& vix quidem difcernendi, jam magno cum e- 


\section{$-\mathrm{XI}-$}

Quod fi ideo plura commutavi nomina trivialia, ut perturbatrix fpecierum confufio evitetur, hanc arbitror diligentiam eo minus vitio mihi vertendam, quo luculentius patet eam apprime necesfariam fuisfe. $f$ ). lllud parvi momenti incommodum, hinc initio forte oriundum, fubjunctis accuratis indicibus eorum facile omnium, quæ apud Auctores obveniunt, nominum, quæque in ipfa tractatione idemtidem occurrunt, omni ex parte levari, dicam an penitus tolli, in. ficias iturum credo neminem.

Quum in Editione LINN压 Syftematis Nature Gmeliniana magna admodum Lichenam cohors, partim earumdem fpecierum fub diverfis nominibus multoties fefe offe: rat, partim fub ineptis dianomis allata reperiatur, utile immo \& necesfarium exiftimavi, has ceterasque, a recentioribus no-

mina-

molumento, fecundum certisfima Natur $x$ indi. cia in plura \& plane diftincta genera dispefci posfunt \& debent.

f) "Nomina neminem terreant nova, fed fi ea "minus placuerint nova ipfe fingas, vel fyno"nyma allegata retineas, fi Tibi magis arride"ant." I.INN $\mathbb{E} U$ S. - Melius certe elt, fcriplit olim amicus b. m. EHRHART, in litteris ad me datis, quod plura unius 'peciei nomina fint, quam ut fpecies ditinct eodern nomine defignatie inveniantur. 
minatas, fpecies, in Svecia licet adhuc non bifervatas, in legitimum redigere ordinem. ccreo ad finem cujusque Tribus, poft illas fvecanas has etiam commemoratas invenies. Siguidem mihi confesfum non fuit has omres ac ingulas meis cernere oculis, pariser atque alias, $a b$ antiquis Scriptoribus, MORISONO, MICHELIO, DJLLENIO, VAILLANTIO, HALLERO, ceteris, delineatas ac defriptas, quas ideo in medium proferre non potui; eis adeo certos \& difinetos characeres adjungere non fuftinui, us isdem tuto dignofcantur \& ceteris omnino pares iudicentur. Novis autem, quas ipfenet vid: diferentiam addere fpecificam meoque judicio fat completam haud neglexi. Quod fi panllo major perfpicuitas atque accuratior or to fyltematicus in totius hujus seneris hucusque nom natis fpeciebus rite, ut decet, cognofcendis, hinc obtineri poffet, mihi omnino gratum foret perque jucundum, quatenus videlicet meam quoque huic negotio fymbolam addere licuerit. $O b$ rationes tamen allatas, non nifi illis fpeciebus certum tribuere locum audeo, quas oculorum obtutui fubjeci \& fedulo examinavi, quasque afterifco (*) etiam notatas volui; ceteras autem crucis figno $(t)$ diftinEtas obtuli. Has inter permultæ forfan reperiuntur, quas veluti aliarum fpecierum varietates fuo jure habeas oportet.

Liche- 
Lichenum partes, fruetificationi corumdem dicatæ, feu quæ, inftitutis non nullis obfervationibus, tales faltem dijudicentur, communi nomine Receptacula appellavi. Verum enimvero quoniam hæc, fitum atque figuram fi fpectes, permultum a fe invicem differunt, e re omnino eft Terminos explanare, quibus næc difcrepantia clarius patebit, quorumque ufum in hoc libello necesfarium pultavi. Verbi gracia:

Scutellce, frequentisfime tam in cruftaceis, quam in foliaceis atque caulefcentibus Lichenum fpeciebus obviæ. In ipfa infantia quafi abfconditæ, dein minutisfimæ \& fere fubglobofæ excrefcunt, impresfione notatæ punctiformi centrali, fenfim dilatanda in Difcum coloratum, vel conftanter concavum \& planiufculum, vel per æatatem convexum \& hemisphæricum; Margine, diverfo modo formato, \& crufta, folio vel caule, concolore, turgido vel tenui fubinflexo, cinctum; Vel per totam bafin fesfiles, vel atiam centro fubtus affixæ, marginibus omnino liberis obfervantur. Exceptio eft in Urceolariis ubi ab infantia \& fem. per concava, inque ipfa fubftantia crufta immerfæ inveniuntur.

Pelta, fpeciem quidem fcutellarum quodammodo eamdem præbent, fed neque eQ. 
eodem modo ab initio explicantur, neque poft lapfum membranulæ, qua in prima juventute teguntur, ejusdem prorfus funt indolis aut formæ. Infignem in Peltideis, quarum in lobis elongatis antice vel poftice affixæ vel potius fecundum totam fuam expanfionem quafi adglutinatæ funt, non raro acquirunt magnitudinem. Figura: fubrotundæ transverfim oblongæ \& reniformes. Superficie: planæ, glabræ, margine tenuis. fimo circumfcriptæ, folio affixæ \& macula quafi coloratæ fpeciem præ fe ferentes.

Tubercula a Scutellis in eo pracipue recedunt, quod non ejusdem originis, quod. que Bacillis fuffulta atque in his terminalia fint; femper fubglobofa \& quafi fungiformia, vix marginata vel margine reflexo; fxpe conglomerata. His inftructa fune: Boeomycides, Calicia, Ifidia, Scy. phophori, Helopodia, \& Cladoniæ.

Ciftell ce funt receptacula globofa terminalia tunicata, fubftantia pulverulenta omnino repleta \& in nucleum compacta, qua tegmine per ætatem rupto \& pulvere elapfo cava reperiuntur. In Sphærophoris tantum occurrunt.

Tha la mi audiunt receptacula fubrotun. da, intra cruftæ vel folii fubftantiam occul- 
ta, ad fuperficiem, quam elevant \& tumentem reddunt fatis confpicua; poro plerumque pertufa vel per xtatem hiantia, intus Thecas a Cl. HEDVIG \& SCHRADER defcriptas foventia. Verrucariæ \& Endo. carpa his funt prædita.

Livella dicuntur Opegrapharum receptacula, forma quam maxime variabiles, facile tamen dignofcendæx, rima longitudinali impresfa intra margines collaterales parallelos elevatos dehifcente. Sunt vero fimplices \& fubrotundæ, oblongæ , lineares, rectæ \&. flexuofæ, vel etiam ramofo compofitæ, ad cruftam femper fesfiles, plerumque tumidæ glabræ, nudæ, rarius membranula tectæ, pulverulentæ, aut depresfæ, conftanter tamen atræ.

Trica $g$ ) folis Umbilicariis pro. priæ, pro diverfa ætate figuram mire ludentes. Jam enim fcutelliformes funt aut turbinatæ, poro fimplici interdum pertufa, vel planæ, marginatæ; jam hemisphæricæ, fubglobofæ, triangulares \& polygonæ, plicis jam concentricis circinatæ, jam, quod frequentisfimum eft \& fingularem harum indolem probat, rugis vario modo plicatis \& gyrofis pulchre ornat $æ \&$ quafi compofi-

ta

8) Gyromaza PERSOONII; Sphara olism mihio 
tæ deprehenduntur. Color etiam in his femper aterrimus.

Glomeruli mihi funt hemisphæria illa pulvere conglomerato conflata \& hoc fuffufa, quæ ad cruftæ vel foliorum fuperficiem fesfilia animadvertuntur, horumque latera, margines \& apices occupantia; in Variolariis pracipue atque $\mathrm{Phy} f \mathrm{fi}$ is, nec non in Imbricariis, Lobariis, Setariis, Usneis \& aliis detegenda.

Cyphella, foveolas minutas margina. tas fcutelliformes etiam convexiufuias ad paginam averfam foliorum fesflles in $\mathrm{Sti}$ ctis repræfentant.

Bacilla denique ut fpeciem fulcri propofui, eodem fere fungentia uffico ut in plantis peduncuii, fcilicet guod liciorum in. ftar Lichenum quorumdam receptacula elevent \& fuftentent; funtque aut folida, \& fimplicia in Boeomycide atque Calicio, e quorum crufta exfurgunt; aut fubdivifa \& fuperne interdum pervia in Helopodiis; aut fiftulofa turfum plerumque dilatata \& fupra claufa in Scyphophoris, vel etiam acuminata \& ramofa quafi freciculofa in Cladoniis, e foliis ipfis apud hos omne Lichenes progenita \& excrefcentia. 
Quod amplius ad Lichenum pertinet naturam five reliquas proprietates certior utique etiamnum defideratur notitia, immo \& experientia fallere nefcia. Eos vero indolis Polyporum hand incerta prabere veftigia \& a Plantis cerre multismodis recedere, variis fuffultus obfervationibus palam fateri atque etiam profiteri haud equidem dubito $h]$. Nonnulla huc pectantia \& attentione fortasis diona, eciam quod ad partes eorum conftitutivas adtinet, atque colorem causfasque varietatun, quibus præ aliis obnoxii funt, ficut \& guidquid commodi illine oriatrr ad fpecies rite difcernendas, alio loco haud ita pridem expofui $i]$. Interea hoc, L. B. parvun fervabis, donec majora parabis.

Vadfeni ex Mureod. 20. Febr. I798.

h) Natura ipfa fociat of conjungit Lapides of Plantas, Piantas \& Animalia; hoc faciendo non connectie perfeltisfimas plantas cum animalibus maxime imper. fectis dittis, led imperfecta animalia or imperfectas plantas combinat. LINN无US.

i) Anmarkningar rórande Lafarterne. Kongl. Vet. Acad. Nya Handl, 1796. T. XVII. p. 206. feqq. 


\section{NOMINA AUCTORUM}

\section{E'I SCRIPTORUM CITATA.}

At. Ac. Sc. Sv. - Kongl. Svenfka VetenR. Academ. Nya Handlingar.

4.. Kied. Sn. - Afta Medicorum Svecia.

12. Nidros. - Det Kongl. Norfke Widenfk. Selfk. Skrif. ter.

At. Palat. - Hiftoria \& Comment. Acad. El. Scient. The. odor-Palat. Voll. I $\rightarrow$ III.

Ar. Soc. Linn. Lond. - Tractfactions of the Linnean So. ciety Vol. I. II.

AZt. Soc. Sc. UpS. - Nova ACta Societatis Scient. Upfal. Vol. IV.

$A f z$. A. Afzerius. Obfervat de Vegetab. Svec. \& in Novis Act. Ac. Sc. Svec.

Allinu. - C. AlLioni. Flora Pedemontana.

Fiarrel. - J. BarRELIER. Icones.

Batsch. - J. G. C. B A T s с H. Elench. Fungor. T. I. - III. Baung.- J. C. G. Baumgarten. Sertum Lipficum. Bellardi - L. Bella RD I. Appendix ad Flor. Pedem. Betfch. Berl. N. Fr. $\rightarrow$ Befchäft. der. Berl. Gefellf. Na. forfch. Freunde.

Blackw. - Herbarium Blackwellian.

Breyn. - J. BREYNIUs. Centur. Plantar.

Bull. - Buld a a D. Herbier de France.

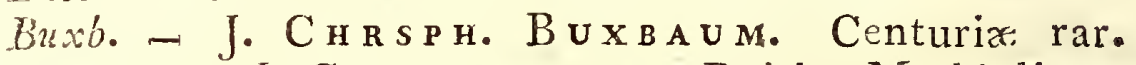

Camer. - J. Camerarius. Epith. Mathioli.

Clus.- C.Clusius. Plant. Hiltor. rariorum:

Colum. - F. C oluman. Ecphrafis. Phytobafanos.

Comm. Lips. $\rightarrow$ Comment. Lipfienfes.

Crantz. - H. J. N. C RANTz. Inflit. rei herbar?

Davies. - Hugr. Davies. In Act. Soc. Linn. Lond.

Deut. Mag. - Deutfche Magazin.

Dicks. - J. D I C k s o N. Plant. Crypt. Britan. Fafc. I. II. III. Dillen.- J. J. D I L e N I U s. Hiftoria Mufcorum.

Doerr. C. H. DoerRIEN. Verzeichnifs Oran-Nasfal gewächfen.

Ehrh. $\rightarrow$ Fr. E H R ART. Beytr. zur Naturkunde \&c.-, Plantæ Cryptog. exficcatæ. 
Fl. Dan.- Floræ Danica Icones.

Grr. - L. C. G r R A R D. Flora Provincialis.

Geuns. - S. f. van GEUN s. Plant. Belgic. Spicileg.

Gle $\rightarrow$ J. G. Greditsch. Syft. Plantar.

Gmel. J. F. GMELIN. Ed. I3. Linnæi Syft. Naturæ.

$\rightarrow$ Snumer. Stirp. Tubingens.

Gort.- D. Gorter. Flora Geldrica. - Ingrica.

Gronov. J. F. Gron ovius. Flora Virginica.

Guett.- J St. Guet t a R D. Obferv. in Mem.d. 1'Acad.

Gunn.- ソ. E. Gun n e RUs. Flora Norvegica.

Itäg. C. G. HA G N. Hiftor. Lichen. Prusficor.

Flul! - A. v. HALLER. Hiftor. Stirp. Helveticor.

Hedo. - J. HEDw I . Fundam. Hiftor. natur. Mufc. - Stirp. Cryptogam.

Hoffh. - C. HOFFBRG. Anvisn. til Växt-Rikets kännedom.

Hoffm. - G. F. Hof F M A . Enumer. Lichenum.- Plan. tæ Lichenofæ. - Deutfche Flora - Veget. Cryptog.

Houtt. - Hou T TUYN. Pflanzen Syftem.

Huds. - Gu I L. Hudson. Flora Anglica.

Humb. - F. A. $a b$ H и м в ог о т. Spicileg. Florx Friberg. Facqu. $\rightarrow$ N. J. J A cev I n. Collect. ad Hift. Natur. fpectant. - Mifcellan. Floræ Auftriacx.

Kniph. - J. HIERON. KN I PHOF. Botan. in Orig̈inali.

La Mark. J. B. DE LA MARK. Flore Françoife.

La Tourette- A. L. LA Tourette. Chloris Lugdunenfis.

Ieers. - J. D. LeERs. Flora Herbornenfis.

Leys. - J. W. LeYser. Flora Halenfis.

Lightf. - J. L I G н T F Oот. Flora Scotica.

Liljebl. - S. LrLJEBLA D. Utkaft til en Svenfk Flora. Ed. I.

Link. - H. F. L I N K. Annálen der Naturfchichte.

Linn.- C. à L inné. Syftema Naturæ- Flora Svec. \& Lappon.- Species \& Mantisfæ Plantar.

Loes. - LoEselius. Flora Prusfica.

Lumnitz. - St. Lumnitzer. Flora Pofonienfis.

Mattufc.- H. G. v. Maтт т sснка. Flora Silis. Stirpes Sil.

Michel. - P. A. M г с н El I. Nova Genera Plantar. Mohr. - N. Mон R. Forfög til en Islandfk Naturhiftorie. Moris - R. Mo R I son. Hint. Univ. Plant. Oxoniens. Mull. - O. F. Mul L E. Flora Fridrichsdal. \& in Deut. Magaz.

Murr. $\rightarrow$ J. A. MurRaY. Prodrom. Defignat. ftirpium Gottingens. 
Wunch. - C. Mö N Cr. Enumer. Plant. Hasfix.

Weck.- N. J. d. NEckero Methodus Mufcor, - Deli. cix Gallobelg.

Oed. - G. C. Oeder. Enumer. Plant. Norv. Dan. \&C. Olafs. - EGg. Otafsen. Islands Reife.

l'all. - P. S. Pallas. Reifen durch Rufsland.

Perfoon. - C. H. Persoon. Ap. Ufter. in Annal. der Botan.

Petiv. - J. Petruer. Gazophylacii Natura Decades.

Plam. $\rightarrow$ C. PeUMIER. Filices American.

Pnllich. - J. A. Pold I H. Hiftor. Plant. Palat. Elect. Paj. - J. Rásus. Synops. Method. Stirp.

Reich. - J. J. REICHARD. Flora Moenofrancofurtana. Rclian. - R. RelaAn. Flora Cantabr. c. Suppl.

Retz. - A. J. Retzius. Floræ. Scandin. Prodromus. Obf. Botan. Fafc.

Reyz. - G. ReYGer. Florz Gedan. Tentamen.

Rorh. - A. G. Rотн. Tentam. Floræ German. $\rightarrow$ Botan. Magaz.

Roy. A. van Royen. Flora Leydenfis.

Rutfr. - C. B. R uts tröm. Disfert. Novæ Crypt. Svec. Römer. - J. J. Römer. Neue Botan. Magaz.

Scholl. - F. A. Scholler. Flora Barbyenfis.

Sihrad. - H. A. Schrader. Spicil. Floræ German.

Schrank. - F. P. Sch RANK. Primit. Floræ Salisb. Baicrfche Flora.

Scracb. - J. C. I). S с н в в в R. Spicil. Floræ Lipfienf. Senp. - J. A. Scopoli. Flora Carniolica. Sibth. - J. S г в тнов P. Flora Oxonienfis. Sir.n.h. Selfk. - Skrivter af Natur. Hiftor. Selfk. Smith. - J. E. S M Iтн. in Act. Soc. Linn. Lond. Sowerby - J. S ow e r в Y. Englifh Botany. Sruartz. - O. Sivartz. Meth. Mufc. emend. - Nov. Act. Upf $\rightarrow$ Prodr. Plant. Ind. Occid.

Tabcrn. - J. Th. Ta bernemontanus. Kräuter Buck. Thunb. - C. P. T н U в в R G. Flora Japon \& Capenf. ined. Tode - H. J. TodE. Fungi Mechlenburg. fel. Tournef, - J. TOURNEFOKT. Inftitut. Rei Herbarix. U, - P. Usteri. Annal. der Botanik.

Vahl. - M. VAнL. in Flora Dan. \& Skr. n. h. Selfk. Vail!. - s. VAillant. Botanicon Parifienfe.

Velent. - Bas. VALENTIN. Mufeum.

Villars - L. M. De V I L a Rs. Hift. des Plants de Dau. phiné.

iicb. G. H. W e в ER. fpicil. Floræ Gottingenf. H'cig. - C. E. W é a e L. Flora Pomer. Rugica \& Suppl. $\rightarrow$ Obferv. Botan. 


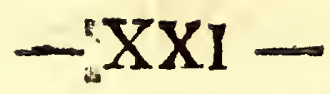

Weis - F. G. We r s. Plantx Cryptog. Flor Gottingenf. Weftr. - J. P. W Es Tr IN G. in Nov. Act. Ac. Sc. Svec. Wigg. - F. H. W I Ggers. Primit. Floræ Holfat.

Wild. $\rightarrow$ C. L. WILDENow. Floræ Berol. Prodr. Botan. Magaz.

Wither. - W. W I Thering. A Botan. Arrangem. of Britifh Plants.

Wulf. - F. X. a Wulfen. in Befch. Berl. N. Fr. \& apud Jacqu. in Collect. \& Mifcell.

Wulff. - J. WuLFF. Flora Borusfix. 


\section{- XXII - \\ EXPLICATIO FIGURARUM.}

\section{Tab. I.}

\section{Fig. I. Lichen Dillenianus.}

a. Crufta magnitudine naturali, rugofo plicata, crasfa, coacervatim \& inæcualiter in lapide proveniens, fubcarneo rofea; Scutellis pasfim congeftis cafio pruinofis nigro marginatis.

b. Pars cruftre feparata magnitudine aucta, quo plicx ejusdem evidentiores redderentur.

c. Acervulus crufte cum fcutellis quibusdam confertis, magnitudine adaucta.

d. Particula crufte cui fcutelix fparfim affixx fubangulof magnitudine naturalem multum fuperante.

Fig. 2. Lichen Swartzii.

a. Crufta naturali magnitudine lapidis frufulo orbiculatim \& arde affixa, lavis contigua vix rimofa alba; Scutellis confertis fubglobofis glaucis.

b. Marginis crufte fimbriati fragmen in .lapillo difcisfo, oculo armato vifum.

c. Pars crufte cum fcutellis per xtatem aggregatis \& inxqualibus cruftam fere obtegentibus, magnitudine naturali.

d. Cruftx vetuftx particula, fcutellis demum angulofis \& marginatis obfesfa, magnitudine aucta.

\section{Tab. II.}

Fig. 1. Lichen Ehrhartianus. 


\section{- XXII -}

a. Crufta magnitudine naturali ad fuperficiem corticis Quercus indeterminatim effufa albo vi. refcens, verrucis pundiformibus rugofis nigris adfperfa, fcutellisque flavefcentibus inæquali. bus fparfis \& conglomeratis.

b. Crufta junior tenuis inxqualis grifea, verrucu. lis nigris punctata nec fcutellifera, qualis in lignis vetuftis pracipue occurrit.

c. Pars crufte mature ad lentem vifa, plic's pápillofis compofita, in quibus pasfim verructs rugof $x$ atræe adpofitze obfervantur.

d. Corticis particula crufta Lichenis ex parte teAa, in qua Scutellæ, ope lentis, auctre, tam juniores minutæe rotundatæ \& marginatæ fparfx, quam vetuftiores congeft $x$ inæquales ac flexuo. fæ fere immarginatx affixæ funt.

\section{Fig. 2. Lichen Weftringii.}

a. Crufex pallide rimofe \& inacqualis lobulus irregularis in lapide expanfus, magnitudine naturali.

b. Pars crufte ramulofx per lentem adfpecta.

c. d. e. Ramuli fimplices \& ramulofi pallidiores \& fubalbiải apice concavi vel Tubercula fufca gerentes, a crufta feparati \& magnitudine multum aucti.

$f$. Acervulus fere hemisphæricus e ramulis erectis aggregatis confertisfimis faftigiatis compofitus, quaiis pasfim in crufta obfervatur, magnitudi. ne naturali vix aliquantum major.

g. Confimile tuberculum $\mathrm{l}$. hemisphærulum magnitudine pluries auctitatum, crufta ejusque ramulis folito more dispofitis, huc \& illuc verfis, cinctum.

Oblatam gaudeo mihi optatisfimam occafionem, meam Viris Clarisfimis debitam teftificandi \& venerationem \& gratiam, qui de Lichenum hiftoria optime meriti funt, Eo. 


\section{- XXIV}

rum, DILLENII puta, EHRHAR TI, SWART TII, WESTRINGII nominibus hifce nimirum \& fpeciebus imponendis, ut, quamdiu Lichenes umquam progerminant atque leguntur, tamdiu Horum non minus, quam Reliquorum, nomina, quorum fingulis fuis fpeciebus adfcripta in prafenti enumeratione funt, renovetur memoria.

Icon titulo hujus Prodromi adjecta $L i c h$ enem $A c h a$ $r i i$, quem ob benevolentiam \& amicitiam in me fingularem ita nuncupatum voluit $\mathrm{Cl}$. WESTR ING, magnitudine naturali, in granitide crefcentem fiftit, crufta orbiculatim expanfa duriufcula contigua levigata \& vix tenuisfime rimofa, pallide lateritia. - Figurze tres minores fub. junctx Scutellas hujus fpeciei fparfas rubellas excavatas cum crufte fruftulis $f$. areolis in quibus impresfer funt, formamque earumdem pro ratione ætatis tantifper diverfam offendentes, oculo armato, magnitudine plus minusque adauctas, commonfrant. 


\section{- I. - \\ DIANOME LICHENUM.}

Omnia qua a nobis vere dignofci. posfunt, dependent a slara methodo, qua diffinquimus finilia a disfimilibus. Hac juethodus "quo magis naturales comprehendit diffinetiones, co clarior remu mobis naficur idas. Qus circa plura verfatur nofter conceptus objecta, eo difficilior elaboratur sinethodus, at sangis necesfaria evadit.

\section{LINNRUS。}

\section{FAMIL1A. I.}

LICHENES CRUSTACET. Cruftacea qualicumque expanfione notabiles.

A. Crufta pulverea aut flocculofa irregularis.'

I. LEPRARIA, Receptacula vix ulla detecta.

B. Crufta folida planiufcula uniformis fuborbicularis.

a. Receptacula inclufa feu abfcondita, ex pares ad fuperficiem iubernfo-prominestia.

2. VERRUCARIA. Thalami crufte fubtantix innati lubd rotundi poro notati vel pertufi.

b. Receptacula ad crufe fuperficiem resfilia.

3. OPEGRAPHA. Lirelle crufte adnatx varix, rima longi. tudinali dehifcente.

4. VARIOlaRIA. Flomeruli crufte adnati fuperficiales pulverulenti.

5. URCEOLARIA, Scutella crufta areolis \& verrucis immerfax \& excavatz.

6. PATellaria. Scutelle crufte adnata fuperficiales, aue concavx \& planx marginatx, aut convex $x$ hemifphxricx vix marginatx.

c. Receptacula in bacillis terminalia.

7. BOEOMYCES,' Tubercula fubglobofa fungiformia xqnabilia: A 
8. CALICIUM. Tubercula fublentiformia fuberofa, difco pulverulento elevato.

C. Crufta ramulofa inæqualis, ramulis coralloideis confertisforis compolita.

9. ISIDIUM. Tabercula terminalia.

D. Crufta fubfoliacea.

a. Crufta irregularis fquamolo-imbricata.

10. PSOROMA. Siutelle laterales \& marginales.

b. Crufira adpresta plana orbicularis, margine lobaio perfiftente.

I. PLACODIUM, Sctitelice laterales.

\section{FAMILIA. II.}

\section{LICHENES FOLIACEI.}

Acaules, folium fesfile fimplex feu muliplicatum laciniatum fubmembranaceum exhibentes.

A. Receptacula in foliis fesfilia fparfa.

a. Folia membranacea plano-depresfa in orbem dispofita imbricata lubtus fibrillo/a.

12. IMBRICARIA. Siutelle \& Glomeruli laterales.

b. Folia gelarinofa lohata imbricata vel diffufa.

13. COLLEMA. Sicutclla laterales \& marginales.

c. Folium fubcartilagineum depresfum fubpelia. tums.

* Receptacula intra folii paginas abjcondita protuberantia.

IA. ENDOCARTON. Thalami fuỉtantize folii innaci, oftiolo denum hiantes.

* Receptacula fesfilia fuprafoliacea.

15. UMBILICARIA. Trica gyrófo-plicatx.

d. Folia fubcoriacea expanja vaga adfcendensia fubtus villofa. 
* Folia fubcoriacea expazara late lobata fape lacunofa fubrus hivia.

16. LOBAR!A, Scutella \& Glomeruli laterales.

* * Folia fubcoriacea, fubenes Cyphellis inua

$$
\text { lanteinem pariss. }
$$

17. STICTA. Siutella Iparfix vel Pelte marginales.

* * Folia coriacea, fabtus plerumque to. mentofo-venofa.

18. PELTIDEA. Pelta larerales \& fubmarginales, antica vel pofticx.

e. Folia membranacea adcendentia crifpa u. tringue nuda.

19. PLATISMA. Scutella pelriformes fparfe.

f. Folia fubcartilaginea glabra caspirofa canaliculata vel plana linearia of ramojo- 18. ciniata.

20. PHIYSCIA, Scurelle fparfe.

B. Receptacula a foliis remota in bacillis terminalia.

a. Folia membranacea rigida laciniata fcyphifera. 21. SCYPHOPHORUS. Tuhercula in bacillis fiftulofis fuperne dilatatis claufis terminalia.

b. Folia membranacea rigida minuta fubimbricota in bacillorum ramulis tuberculifera. * Bacilla folidinfrula Superaze vix dilataia fiasspliciter fuldivila.

22. HELOPODIUM. Tubercula fungoh terminalia.

* * Bacilla elonsata tubulofa fuperne arzensa. ta fruticulofo - ramofa.

23. CladONIA. Tubercula fubglobofa terminalia.

\section{FAMILIA III.}

\section{I,ICHENES CAULESCENTES.}

Caules tolidos ramofos teretes fruticulofos, vel LOO ra elongaca proferentes. 


\section{$-4 \cdot-$}

A. Fruticulof, caulibus folidis rigidis.

24. STEREOCAULON. Scutelle fpharoidex folidx fparfa. 25. SPHAROPHORUS Ciftella tandem dehifcentes terminates. 26. CORNICULARIA. Scutella radiata demum convexiufcula terminales.

B. Filamentofi, loris elongatis. a. Lora nuda glabra contigua.

2\%. SETARIA. Scutelle \& Glomeruiti laterales.

b. Lor a cortice cruflacea veftita fubariculara fibrillofa.

28. USNEA. Scutella planx radiatx vel convexa fubmarginawe \& Glomeruli iparfi. 


\section{$-5 \cdot-$}

-

Cognitione Specierun innititur omsis folida ereditio Phy.

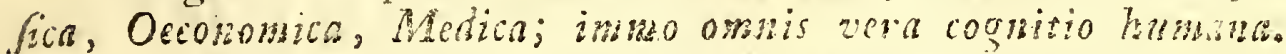

LINN. Prilof. Bot.

\section{I. \\ LICHENES CHUSTACHI}

\section{Tribus I.}

Lepraria. Svet. Pulverlafvar.

Crufta pulverea effura diformis; rarius etian focculorofubfibrillofa.

Receptacula nulla manifelta, vel adhuc non certo in. dagata.

1. LICHEN antigritatis - cruftaceus pulverulentus tenuis ater.

Lepraria antiquitatis. Svet. Oldlaf.

Lichen antiquitatis Screb. Spicil. Hagen, Reich. Fl. Roth. Tent. Gmel. Syft. Linn. Hoffm. Enum. Lich.

Lepra antiquitatis Wigger. Primit. Fl. Holf. Schrank. Prim. Sal.

Bysfus antiquitatis Linn. Retz. Prodr. 2. Liljcbl. Sv. Fl. Hoffb. W. R. Huds. Neck. Delic, Mattu/c. Leers. Lumnitz. Wither. Arr.

Icon. Hoffm. En. Lich. T. 3, f, 5. Dillen. Hift. Mufc. T. I. f. 18.

Habitat fupra rupes \& lapides, in muris \& te. gulis antiquis.

Obf. Nullus dubito quiso Auclores nonnulli jub no. 


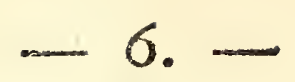

mine L. nigri hune esiam intellexerint. Hine fynowme fatis incerta Junt.

2. Licien fegefria - crufraceus pulverulentus temuis cinereus.

Lepraris Jegefiria, Svet. Stroolaf.

Lichen fegefiria Neck. Meth. Murc.

Lichen cinereus Bag. Roth. Tent. Gmel. Sylt. Linn. Lichen antiquitatis var. B. Hoffm. Fnum. Lich. Eysfus faxatilis Linn. Retz. Pr. 2. Hoffb. W. R. Huds. Lightf. Scop. Weis. Reich. Fl. Mattufc. Reyg. Gunn. Allion. Leers. Wirher.

Habitat in faxis \& rupibus.

3. LiCHEN flavis - cruftaceus pulverulentus tenuis flavisfimus.

Lepraria flava. Svet. Guliaf.

Lichen flavus Schreb. Spicil. Hag. Hoffm. En? Lumnizz. Gmel. Sylt. Linn.

Lichen candelaris Neck. Meth. Roth. Tent. Lepra candelaris Ehrh. Crypt. exf.

Iepra candelaria. Wigg. Prim. Schrank. Bav. prim. Sal.

Byclis candelaris, Linn, Retz. Pr. 2. Liljebl. Hoffh. Hudf. Lightf. Pollich. Weis. Mattuse. Scop. Neck. Delic, Reyg. Allion, Gunn. Scholl. Leers. Vither.

Icon. Hoffm. Enum. Lich. T. r. f. 4. Fl.' Dan.

T. 899. f. 2. Dillen. Hift. Mufc. T. I. f. 4. Habitat in lignis fubputridis \& ad corticem vevetufam arborum.

Obf. Conformatio crufte diversa a. L. candelarii varietatibus pulverulentis.

4. LrCHen chlorinus - cruftacens pulverulentus fub. lantuginofus pulvinato conglomeratus mollis fla. visinus. 
Lepraria chlorina. Svet. Flocklaf.

Lichen candelaris Wefring. Nov. Act. Ac. Sc. Svec. 1791. p. 136.

Habitat in fisfuris rupium antiquis \& umbrofis. Ohf. a. Lo aureo diftuctisfimus. Color perfaferas Crufta tumida pulvinata cras $a$.

5. LICHEN latebrarum - cruftaceus pulverulentus fub. lanuginofus pulvinato cong lomeratus mollis grifeus, Lepraria latebrarum. Svet. Kiyftlaf.

Habitat in cryptis umbrolis \& latebris petrarum. Obf, Cum Bysfo cryptarum Limonon confundendus.

6. LICHEN albus - cruftaceus pulverulentus albisimus. Lepraria alba. Svet. Pesderlaf.

Lichen albus, Gmel. Syft, Linn.

Lichen lacteus Schreb. Spicil. Hag. Neck. Koth. Tent. Hoffm. Enum. Lich, Lummitz. Lepra lactea Wigg.prim. Schrank Bav.prim. Sal. Bysfus lactea Linn. Retz. Pr. 2. Hoffl. Weis.

Scop. Pollich. Huds. Lighef. Reyg. Martuslc. Reach. Fl. Scholl. Leers. W'ulf. Schr. Nat. Fr. Vither.

Icon. Hoffm. En. Lich. T. 1. f. 3. Fl. Dan. T. 840. f. 4. Dillen, Hift, Mufc. T. I. f. 2. Habitat ad mufcos \& arborum corticem.

7. Lichen incanus - crufaceus pulverulentus glebulofus fubvillofus incanus.

Lepraria incana. Svet. Hvitlaf.

Lichen incanus. Schreb. Spicil. Neck. Meth. Hag. Hoffm. En. Gmel. Synt. Linn. Roth. Tent. Lumnitz.

Lepra incana. Wigg." Primit. Schrank Bav. Prim. Sal.

Bysfus incana Linn. Retz. Pr, 2, Hoffb. Huds. 
Lighte. Wcis. Neck. Delic. Scop. Reich. Fl. Gisn. Scholl. Wither.

Icon. Hoffm. Enm, Lich, T. T. f, 6. Dillen. Mif. Mufc T. T. f. 3 .

Habitat fupra mulcos ad terram hunidam umbrofam \& corticem arborum.

Obf. Cruftam L. mufcorum ex hoc conftruttam obfervationibus indwbitatis nordum fatis evilum. eq⿴囗十。

8. Lichen argenus - crulraceus contignus rquabilis fubrimofus pulverulentus candidus.

Lepraria argena. Sver. Kritlaf.

Lichen farinofus Hoffm. En. Lich. Gmel. Sylt. Linn.

Vermearia farinofa Hoffm. Deut. Fi.

Icon. Hoffm. Enum, Lich, T. I. f, I. Mich, Gen. T. 53 . . 4.

Habibat in cortice arbonm.

Obf. Scritellas pallidas hujus crufte ainatas fe vidisfe refert Hoffm. Desst. Fl. 2. p. 171 .

Hec Specierem Trigis (L. albus, incanus, ar: genus) arko nimis vinculo inter fe coharet of egre charactere diffinguitur. Lenti autem fubjedit hi Lichenes, guod ctiam ex Hoffmanni figuris fatis apparet, a le invicem, refpectu conformationis pariixms crufta, certe difcrepare videntur.

9. Lichen variegains - cruftaceus pulverulentus ruhro \& aibo variegatus.

Lepraria variggate. Svet. Skumlaf.

Lichen variegatus Hag. Relz. pr. 2. Hoffm. Enum. Lich. Gmel. Sylt. I Linn. Icon. Hagen. Hilt, Lich, T. I. f. I. Habitat in frepibus putridis \& Belula albæepidernide. 
Obf. In Jpeciminibus ad Betule albo epidermidem lectis per alignod tempes asfervatis color rubellus 1. carneus friation immixtus non dis perdurat. Madefactus odorcm Jpiras fatis ingratum fubnarcoticums.

10. Lichen rofens - cruftaceus pulverulentus conglomeratus roleus.

Lepraria rofea. Svet. Rorenlaf.

Lichen rofeus Schreb. Spicil. Hoffm. Enum. Gmel. Syft. Linn. Roth. Tent. Scholl Lumnitz. Lichen rofatus? Wulf. ap. Jacqu. Coll. 3. Bysfus rofea. Retz. Prodr. Fl. Scand. 2.

Habitat in arborum cortice antiqua, Salicis, Tilix.

II. Lichen lucefcens - craftaceus pulverulentus granulato verrucofus fulphureus.

Lepraria listefcens. Svet. Sroutsgullaf.

Lichen luteiceins Hoffm. Enum. Lich. Gmel. Syft. Linn, Lumanitz,

Lepra lutefcens Hoffm. Pl. Lich. Schrad. Spicil. Verrucaria lutefcens Hoffm. Deut. Fl. Icon. Hoffm. Plant. Lich. T. 23. f. I 2.

Habitat in cortice Fagi, Quercus, Betulx, Abietis. Obf. Scutellas in hac Jpecie deteltas affirmat Perfoon. Uft. Anmal. d. Bot. St. 14. p. 35., of alias marginatas, demum convexas, fluvo dilutis/ime rufe/centes, margine flexisolo obliterato, a fe inventas defcribit Hoffman Deut. Fl.2. p. 105. An $L$. orolthei noftri? vide infra. $A$ me faltem receptacula nunquam in $\mathrm{L}$. lutefcente ob/ervata. Crufta quafi ex glomerulis hemispharicis pul. verulentis Japisfime compofita, orbicularis, affinitatem quandam cum Variolari is prodit.

Hujus etiam loci forte eft: Verrucaria maculiformis, leprofo farinacea luA 5 
tefcens maculiformis, jcutellis margine conco. lorabus. Boffus. D. Fl.

12. Lreren cocodes crufaceus granulatus fubri. molus murio luscoque varius.

Lepraria coccodes.' Svet. Smalkiaf.

Lepra oblcura Ehrhart. Cr.

Habitat ad cortices \& lapimenta lignea vetulta. Ob́. Hebitus I. Intelcentis. V ariat colore grams. iorum fordide pallido, cintreo, murino, fulco. Cruffa irregulariter effufa.

13. I ICHEN boryoides - cruftaceus pulverúlentus granulatus viridis.

Lepraria fotryoides. Svet. Gronlaf.

Lichen botryoides Hagen. Hoffm. Enum. Roth. Tent. Gmel. Syft. Linn. Lumnitz.

Lichen bystinus Scopoli. Carn.

Lichen virilis Schreb. Spicil.

Bysfus botryoides Linn. Reiz. Pr. 2 Liljebl.

Hoffb. Huds. Lightf. Reyg. Weig. Neck. Delic.

Weis, Pollicin. Scholl. Wither.

Icon. Hoffm. Enum. Lich. T. I. f. 2. Fl. Dan.

T. 840 f. 3. Dullen. Hilt. Mufc. T. T. f. 5. Tabitat in muris, terra, locis udis \& ligno putrefcente.

Obl. Scmellas bervasfe fertur Perfoon. Uf. Anm. d. Bot. St. 14. p. 35. (cfr. L. Erylibe.)

Variat colore late of faturate viridi, flavejcen te, olvaceo \& demum fubpurpurafente, inprimis quando crufta per atatem gelatinola evadit.

Forte hujus tantum varietates funt:

Lichen aruginolus Roth. Tent. Lichen gramineus Gmel. Syf. Linn. Eysfus aruginofa Scholl. Barbiens. Vix nif fentem hujus Lichenis Ratum indigitat: Tremella botryoides Schreb. Spicil. 
14. LiCHeN? amens - lanuginofus fubpulverulentus pulvinatus croceo aureus, $x$ tate canelcens.

Lepraria? aurea. Svet. Guldiaf.

Byslus aurea Linnai. Lightf. Schrank Fl. Bav. \& Prim. Fl. Sal. (?Retz.Pr. 2. Weis. Polloch. Teps. Neck. Meth. Roin. Tent. Gleditfoh Fung. Gmel. Sy(t. Linn.)

Icon. Dillen. Hift, Mufe. T. T. f. 16. Fl. Dan. T. 718. 1. I. Nichel. Gen. T. 89. f. 2. Petiv Gazoph. 1. T. I5. fo 3o

Habitat in Taxis \& mulcis putrefcenibus.

Obl. Ob fimilem cum Lichene gosfypino eexturam, quafi bombycinam, inter Lichones jam exumeravi.

Quedam ex allatis fynonymis ad L. chlorinum, noftrum, forfara, releganda.

I 5; LICHEN jolithus - cruftaceus pulverulento - fubfibrillofus ruber, faxatilis,

Lepraria? jolithus. Svet. Viollaf.

Lichen jolithus Swartz, Ait. Upf. IV. Liljebl.

Hag. Gmel。 Syit. Linn。

Lepra jolithus Schrank. Prim. Sal. Bav.

Byslus jolithus Linn. Retz, Pr. 2. Haffb. Grans.

Matwicka. Wither.

Icon. Fl: Dan. T. 899, f, I, Mich。 Gen. T, 89. f. 3 .

Habitat ad lapides.

Obf. Occurrit mere pulverulentus \& quaf fibrillo. fus. An ille vetuftus, vel pecie ab altero difindius? - L. rubens Hoffra: vix differt.

16. LiCHeN arcumatus - cruftaceus pulverulentus croceus, arboreus.

Lempraria? arcumata. Svet, Luktlaf.

Jichen arcumatus Gmel. Sy?t, Linn.

Lichen croceus Schreb. Spicil. Roth. Tent. Habitat ad corticem Fagi, Qvercus \& 
Obf. An a L. cinnabarino Fag. val I. ruben. te Boffm, diverfus?

+ L, cobaliginess - cruftaceus, impalpabilis ro. feus latisfme fparfus. - Lepra cobaltignea Schrank. Prim. Fl. Salisb. - Bysfus cobal. tiginea Wulf. ap. Jarqu. Coll. 2. T. 12, fo Lepraria cobaltiginea. rup. calc.

NotA. Ab hac Tribu of guidem a numero Lichenum forjan segregande forcut quadam jam recenfita of á Autoribus huc relate Species; $v$. $g$. Lichen botryoides, L. aureus, L. jolithus, L. rubens, $\mathfrak{F}$ que funt relique harnm varietates; L. roleus, L. tuberculofus Hoffm. Enum. Lich. \& L. ferrugineus Ejusd. nec non: L. cinnabarinus Hag. Hift. Lich. - Fungora ut ita dicam quorundam natura ac. Selatinofa fubltantia quando exoleverint, pariteratque aliena textura of conformatio; pracipue vero paries illa, fructificationi corundem, ut Saliem videntur, dicate, forma ina'olis prorfus diveria, huic noftra opinoni rabur addunt haud minimum.-Leprarias eiram religuas, quafi plantas adhuc imperfectas, feus imwaturas, of veluti initia aliorum Lichenum, quadam jure confiderandas esfe, plus quam probabile videtur.

Lichen rubens Hoffm. En. Lich, T. I. f. s. Reich. Fl. Schrank. Veig. Gmel. Syft. Linn. - Lichen odoratus Roth, T'ent: Gmel. Syft. Linn. (L'e. pra odorata Wigg. Prim.) — Lichen putredinis Roh. Tent. Gnel. Syft. Linn. (Lepra putredinis Wyg5. Prim.) forte varielates ejusdem (peciei funt; colore rubro, miniate $\mathcal{F}$ purpurafcente, atque crufa plus minusgue fugace diverja. Humetiati foveolent. 
Per atatem color evanefcit uti in L. jolitho, of se vera inter $f_{e}$, vel ab eo, difincti fint hi omnss tuto affirmare non posfumus.

In crufia fubfloccubla L. jolithi ague ac L. ru. bentis conigit etiam mihi aliquando (cutellas pedicellatas turbinatas albas (Nov. Ait. Up\%.IV.) pulverulentas videre, fed nefaus an his propria fivot, net. se, Leprariis jam cunjunxi.

\section{Tribus 2.}

VERrucaria. Svet. VÅKtLAFVAR。

Crufta folida diffufa fuborbicularis uniformis plerumque tenuisfima \& fubnisida.

Receptacula: Thalami fubrotundi, minuti, in ipfa cruftx fubftantia frequenter immerfi, fupra eam parum elevati", ineus cavi $l_{\text {. }}$ pulverulenti \& fructificantes, poro fupra notati l, pertufe, rarius vel tantum in infanria claufi,

17. LICHEN Schraderi - crultaceus albefcens lævisvisfimus; thalamı fubimmerfis umbilicatis atris. Verrucaria Schraderi. Svet. Schraderslaf.

Lichen rupeltris, var. 2 Scop.; var. $\gamma$ Roth.Tent,; var, $\gamma V_{\text {eb.; }}$ var C. Hoffm. Enum. Lich. Lichen petrofus $G$ mel. Syft. Linn.

Lifchen fufcoater. var. B) Hagen Hift. Lich. Verucaria rupeftris. Schrad. Spicil. Vigg. Prim.

Verrucaria immería Perjaon Utt, Ann. d. Bot. St. 7.

Verrucaria Weberi? Humbolt. Spicil. Frib. Icon. Schrader Spicil. Fl. Germ. T. 2. f. 7. Habitat in laxis calcareis, paslim etiam in arenariis. 
Obf. Cum L. immerfo v. T. l. neútiquam confundenda Jpecies nti perbene demonftravit $\mathrm{Cl}$. Schra. der $l_{0} c_{0}$

18. Lichen grifeus - cruftaceus rimofus grifeus; thalamis immerfis nigris.

Verrucaria grifea Sver. Grâlaf.

Verrucaria grifea Perjoon Ult, Annal, d, Bot. St. II.

Habitat ad rupes.

19. LICHEN entypas - cruftaceus albocinerafcens lavisfimus; thalamis totis immerfis difco in. presfis nigris.

Verrucaria emtypa. Svet. Sänklaf.

Habitat fupra lignum in truncis Fraxini antiquis cortice denudatis.

Obf. Similitudo multa cum Mucilagine reticulata Hoffm. Spharia affinis.

20. LICHEN umbrinus - cruftaceus fufcoater lævisfimus, te uisime rimolus; thalamis conicis fubpapillofis concoloribus.

$V$ errucaria umbrina. Svet. Skugglaf.

Lichen fufcoater var. a Hag. Weis.

Verrucaria nigrefcens Pirfoon. Uti. Ann。 St. I 4. Habitat ad faxa \& lapides crufta parca arcte affixa.

Obf. Color umbrino fulcus per atatem omnino nigrefcie. Thalamos, qui conoidei of apice ra. rius poro notati, nunguain hiantes vidi. Verosimile fatis videtur, quamplurimos $A u$. Etorum hunc pro L. antiquitatis \& L. fufco. atro venditas $\int e_{\text {. }}$

2I. IIICHEN picinus - cruftaceus granulato fubimbricatus nigrofufcus; thalamis fubrotundis atris. 
Verrusiaria picina. Svet. Sotlaf.

Habitat in faxis fubftrata terra ex mufcis pu. trefactis.

Obf. Valde fingularis ob cruftam, que oculo armato ex granulis fublobutis imbricatis compofzta obfervatur. Thalami menuti Jupra cruftam Jpar/z Jubglobo/ puncto verminali exiguo impresso notati.

22. Lichen figmaiellus - cruftaceus cinerafcens'tenuis levislimus; thalamis convexis nigris. Verrucaria firgmatella. Svet. Gniflaf.

Verrucaria cinerea Perfoon. Schrid.

Icon. Perfoon. Uit. Ann. d. Bot. St. 7. T. 3.

f. 6. A. Schrad. Spicil. Fl. Germ, T.2.

f. 2. quoad thecas.

Habitat in cortice arborum femimortuorum, ramul. Populi,

23. LICHEN"análeptus - cruftaceus olivaceus tenuisfimus glaber; thalamis globofo fubconicis nigris.

Verrucaria analepta. Svet. Quistlaf.

Verrucaria olivacea Perfoon. Schrad.

Icon. Perfoon Uft. Ann. d. Bot. St. 7. T. 30

f. 6. B. Schrad. Spicil Fl, Germ. T.2. f. I. quoad thecas.

Habitat ad corticem lævem \& teneram in ar. borum ramulis,

24. Lichen melaleucus - cruftaceus albus lævis inæ. qualis; thalamis fubglobofis nigris. Verrucaria melalenca. Svet. Punktlaf.

Verrucaria alba Schrad. Spicil. Fl. Germ, Icon. Schrad. 1. c. T, 2, f, 3 quoad thecas. Habitat in cortice tenera arborum. 
25. LICHEN dryinus - cruftaceus albicans pulver!o lentus; thalamis convexiufculis rugofis nigris. Verrucaria? d'ryina. Svet. Eklaf.

Habitat in corticc Quercus antiqua, rimofa. Obf. Habitus Verrucarice, ob cruflam effufam micantem, quamvis Jubpulverulenta adpareat. Receptacisla quadam Julgglobofa apice impres $f_{a}$,re. liqua foutelliformia, propinquizatem cum $P$ atel. lari is indigizant. Craffa contrita $\dot{\sigma}$ humeitate crocea evadit.

26. Lichen ficticus - cruftaceus cinerens tenuisí. mus inæqualis; thalamis fubglobolis nigris. Verracaria fitetica. Svet. Smullaf. Habitat in Tilize, Salicis \& Fraxini cortice.

27. Lichen epidermidis - oruftaceus niveus tenuisfimus lævis; thalamis minutisfimis convexiufculis atris.

Derrucaria epidermidis. Svet. Nafverlaf.

Habitat in tenera epidernide Betulie albx. Obf. Cruftd tenuisfima fere nulla, interdum fubcinerafcens. Thalami minutisfimi fubrugofi.

28. LICHEN atomarius - cruftaceus glaucefcens tenuisfimus nitens; thalamis convexiufculis fufcoatris.

Verrucaria atomaria. Svet. Grandlaf. Habitat in cortice Fraxini.

29. Lichen hyloicus - cruftaceus albus tenuisimus; thalamis fubglobolis fufcis demum nigris. Verrucaria hyloica. Svet. Sidenlaf. Habitat in arborum ligno denudato fubputrefcente. Ob!. Crufta vix ulla. Inter fibrillas ligneas tenuisfimas holofericeas albas emergunt thalami minustisfimi 
tisfimi jam fufci, jam nigri, dilco convero in. negro vel pora impresfo, $A$ L. atomario jat difinctus videtur.

30. Lichen gemmatiss - crultaceus candidus tenuis inaqualis; thatamis hemispharicis atris nitidis. Verracaria gemmaia. Svet, Perllaf.

Habitat in cortice Populi tremult.

Obr. Thalami maltoties majores quam in L. mes laleuco: waturi fere globof or fapins inapertio Habitus L. corticole vel L. nothi.

31. Lichen popalness - cruftaceus cinereo pallidus lavis; thamis tumentibus cæitonigris. Verrucaria populnea. Svet, Afplaf.

Habitat in Populo tremula, Fago, Fraxino. Obf. Inter majores, fo non maximases, in hac Trio bu eflo Puichra é bene difindta pecies, etiam in Indo Occ. a $\mathrm{Cl}$. Swartz lecta.

32. Licuen perturus - crufaceus cinereo virelcens; thalamis contertis hemifphæricis levigatis concoloribus poro uno alterove pertufis. Verrucaria? perisfa. Svet. Porlaf. Lichen pertulus, Linn. Retz. Pr. 2. Liljebl. Lighef. Leers. Pollich. Reich. Fl. Hag. Hoffm. En. Schrank. Alioni. Roth. Tent. Gmal, Sylt. Linn. Lamnitz. Wither. Lichen verrucolus Eudf. El, angl. Gmel. Sylt. Linn.

Lichen carpineus Neck.Aet. Pal. 2 Meth. Mufc. Spharia pertufa Weig. Web. Wigg, Icon. Bloffm. Enum, Lich. T. 3. f, I, Fl, Dan。 T. 756. Dillen. Hit. Mufc. T. 18. f. 9. Michel. Gen. T. 32, f. 52. T. 56. f. 2. Habitat in cortice Fagi; etiam ad faxa. B 
Obf. Corpafcule illa Jubglobofa, atra, nitide, quet in fuperficie interdum Jparfa occurrunt, parafitica ominzo funt; De ceitro paradoxe tamen fpeo ceses of of forte proprii generis.

t I. penciformis - cruftaceus macularis rufo brunneus; thatamis minutis unbilicar is nigris. - Verrucaja puncíiformis Perjoon Unt. Ann. d. Bot. Si. 11 .

Verrucaria pundziformis, arb.

t I. tephroides - crufaceus effulus rimolus; thalasuis nigrefcentibus punciformibus, of iolo hiantibus - Endocarpon cinereum Pero foon Uti. Ain. d. Bot. St. 7.

Eurrucaria uphroides. terr.

† L. crypheus - cruftace us cinereus; thalamis in nerlis nigis pertulis tandem prominulis. - Lichen punctifornis Gmel. Sylt. Linn. Schrank. Bav.

Vorrusaria cryphea.

* L. placoth ilias - cruftaceus rimolus inaqua. lis olivace o fufcus; thalamis prominulis a. pice impreslis nigris. - Endocarpon viridu. lum sctrad. Spicil. T. 2. f. 4. Verrucaria placothalla. saxa.

t Le nitidus - cruftaceus albido olivaceus rin mofus; thalamis bemifphæricis atris nitidis.Sphrria nitida.Web. fpicil. Weig.ObC.T.2.f.I4. Verrucaria? nitida. cort. arb.

Nota, Difficalius gridem ob minutiem of fimilitudinem character cruitur in plurmis Verrucaria lpeciebus, 
at primo intuith, ope lentis of ex habitu bene difiris. gunntur. Cum Sphariis analogian fingularem habent o forte aliguc ox jam enumeratis (peciebus ad $\$ p h$ h$r$ ias potius referende. Ab iis vero differmat Verrucarice expanfone cruflacea of receptacutus ( $P$ er it he c jo PERSOONII) irierne non gelattnofis.

\section{Tribus 3 .}

Opegrapha. Svet. Spricklafvar.

Criafla plerumque parca \& tenuisfima, maculam vel ro: tundatam vel irregularem \& effufan, plus minusve 1atam repræfentans.

Receptacula: Livella oblongx, lineares \& varix, e crufta erumpentes \& fuperficiales, nudx vel membranula interdun tectx, rima longitudinali exarata, dehifcentes,

\section{* I. Lirellis fimpliciufculis.}

33. Lichev Perfoonii _ cruftaceus glaber abidus; lirellis fimplicibus immeritis ovatis incifo rugolis. Opegrapha Perfooniz. Svet. Perfoonsiaf.

Opegrapha rupeltris Perfoon. Ult. Annal. d. Bor. St. II.

Habitat al rupes calcarias.

34. LiCHEN nothus - cruftaceus pulverulentus albidus; lirellis nudis fimplicibus difformibus latiuficulis.

Opegrapha notha. Svet, Sveklaf.

Opegrapha lichenoides Perfoon. Ut, Ann, d. Bot. St, 7.

Icon. Perfoon. l. c. T, 2 f. 4, b.

Habitat ad caudices arborum exaridos. 
35. Lichen diaphorus - cruftaceus glaber fordide albefcens; lirellis fimplicibus in zqualibus, minoribus fubrotundis majoribus elongatis flexuofis,

Opegrapha diaphara Svet, Sprekiclaf. Opegrapha varia Perfoon. Uit. Anu.d Bot. St.\%. Habitat in cortice Fag?, Quercus \& fapmentis ligueis.

36. Licuen obform - crutaceus lavis fubolivace us; lirellis fimplicibus fpartis planis rugons fubellipticis difrormibus.

Opegraptia obfcasra. Svet. Dunkellaf.

Opegrapha obfcura Perjoon Uti. Annd.Botst.7. Icon. Perfosn 1. c. T. 3. f. S. B. b. Habiat ad ramos fagineos.

37. Lichens herpeticus - cruftacens, fubrugofus tumelcens fuico olivaceus nigro limitatus; liv relis fimplicibus frars convexis diftornibus. Opegrapha herpetica. Svet. Raformslaf. Habitat in cortice Populi tremulx.

38. Liches fparcatzis - cruftaceus cinereo furcus pulverutentus; lirollis fimplicibus fubrotundaits rugofis.

Opegrapha spurcats. Svet. Fingtrackslaf. Habitat in truncis vetultis Betulæ albæ \& Alni.

39. IICHEN ainers - cruftacens cinereofufcus; lirellis fimplicibus fubglobofis ovalibusque nitidis. Opegrapha alnea. Svet. Allaf. Habitat ad corticem antiquam Betulæ Alni.

40. LICHEN conglomeratus - cruftaceus cinereo nigrefcens maculiformis; lirellis fimplicibus nudis confertis, 


\section{$-21 .-$}

Opegrapha conglomerata. Svet. Gytterlaf.

Opegrapha conglomerata Perfoon. Uit, Ann, d。 Bot. St. 7.

Icon. Perfoon, 1. c. T. 3. f. 4. A. a.

Habicat in ramis Fagi fylvaticx exficcatis。

41. Lichen congeftus - cruftacens cinereus; lirellis fimplicibus obculis fubflexuofis glabris congeftis. Opegrapha congepa. Svet. Klaslaf.

Habitat 11 Betulæ cortice antiqua, Quèrcu.

42. Lichers macularis - cruttaceus niger rugofus; lirellis fubrotundis congeftis maculam nigram referentibus.

Opegrapha macularis. Svet. Flacklaf.

Opegrapha quercina, Perjoor Un, Ann, d. Bot. St. 7 .

Habitat ad ramos querneos juniores,

Obf. Macula crufte plerumque orbicularis nec late effura.

43. LiCHen epiphegus - cruftaceus niger rugofus; lirellis oblong is confertisfimis in maculas la. tas difformes aggregatis.

Opegrapha epiphega. Svet. Stdinklaf. Lichen rugolus Hoffm. En. Lich.

Opegrapha faginea Perfoon Ufi. Ann. St. \%。 Verrucaria rugofa? Wigg. Prim. Icon. Hoffm. Enum. Lich. T. 2. fo 5. Habitat in Fagi fylv, ramulis,

44. LICHEN vulgatus - cruftaceus cinereovirefcens; lirellis fimplicibus linearibus flexuofis converis confertis,

Opegrapha vulgata, Svet, Skrynklaf. Lichen rugoius Linm. Liljebl. $H_{\text {eff }}$ 。

Lichen 
Lichen fcriptus var. pulicaris? Lightf. Roth. Hoffm.

Icon. Hoffm. Enum. Lich. T. 3. f. 2, c. d, f, Habitat in frultulis corticis $P$ ini Abietis.

Obf. Ipfasfranus L. rugofus Linnai hic eft ev ab Ill. Anctore olim mihi datus. Synonyma re. liqua perquarm dubia. Ic. Dillen. Musc. T': is f. 2. \& Mich. Gen. T. 54. f. 2. forfan I. macularis funt.

45. LICHen elatinus - cruftaceus albefcens; lirellis fimplicibus fubfiexuolis ipartis, lineis eievatis longitudinalibus ruyofs.

Opegrapha elatina. Svet. Rafglelaf.

Habitat ad corticis fquamolæ frultulos Pini Abietis.

Obf. Lirello per atatem magna protuberantes rugo. to inaguales, rarius in crupa sparfa, folitaria.

46. Lrchen vulvella - cruftaceus candidus inæqualis; lirellis fimplicibus ovalibus prominentibus concavis intus albo farinofis fpartis. Opegrapha vulvella. Svet. Kyrislelaf. Habitat in cortica Betulx albre.

47. Lichen reniformis - cruftaceus glaber nitidus olivaceus; lirellis fimplicibus fubreniformibus pellicula tectis.

Opegrapha reniformis. Svet, Njurlaf.

Opegrapha reniformis Persoon Uit. Annal.d. Bot. St. 7 .

Icon. Perfoon 1. c. T. 2. fo c.

Habitat ad ramulos Carpini Betale.

48. Lichen rubellus - cruftacens fubpulverulentus rugulofus rubrofufcus; lirellis limplicibus flexuolisque. 
Opegrapha rubella. Svet. Roodlaitlaf.

Opegrapha rubella Perfoon Ufr. Ann。 d。 Bot. St. 7.

Icon Perfoon 1. c. T, I. f. 2. A. a. Habitat ad caudices Fraxini \& Fagio

49. LicheN fignatus - cruftaceus alboglaucefcens 3 lisellis implicibus fubflexuofis planiufculis fpartis.

Opegrapha fignata, Svet. Trycklaf.

Habitat in cortice exarida truncorum Quercus,

50. Lichen epipafus - crufaceus glaucefcens glaber tenuistimus fuborbicularis; lirellis fimplicibus minutisfimis protuberantibus diffor. mibus fparlis.

Opegrapha epipafta. Svet, Slätlaf.

Habitat in cortice lrvi A ceris platanoidis.

$\mathrm{Obl}$. Lirella omnism tenerrima, qua lineolas o puncla difformia reprafentant, oculo armato rugofa, fupra cruffulam ruptam elevata, atque ex ea quafi erumpentes, reila, curvata of haud raro invicem approximate, variam inducuns formam.

SI, LICHEN betulignus - cruftaceus albus tenuisfio mus, lineolis terpentinis limitatus; lirellis fimplicibus rectis fubparallelis. Opegrapha betuligna. Svet. Björklaf. Opegrapha betulina Perfoon UR, Ann。 do Bot. St. 7. Schrad. Spicil. Icon. Perfoon l, c, T, 3. f, 5. A, a. Habitat in libro Betula albza

52. Lichen parallelus - cruffaceus candidus tenuiso fimus; lirellis fimplicibus acuminatis rectis parallelis planiufculis fulciso 
Opegrapha parallela. Svet. Linielaf.

Habitat ad ligna denudata emortua truncormm

Betulax.

Obf. Cratta I. hyloici; diffcile fatis ef di.

etzu, utrum color huius crofte proprius, an li.

gni in guo affingunteser o intra cujess. fihrillas. longitudinales quale emeergant Lirelle, ippis fio brillis parallele, initio linteo fufca derrum fufco. nigricantes.

* $*$ 2. Livellis ramofis。

53. Lichen denigratus - cruftacens albus; lirellis fubimplicibus difformibus prominulis concavis congeftis nitentibus.

Opegrapha denigrara. Svet, Strimlaf.

Opegrapha atra Perfoon Uft. Ann.d. Bot.St. 7. Icon. Perfoon. l. c. T. 1. f. 2. C. c.

Habitat, in ramis Fagi \& Fraxini.

54. Lichen faderelus - cruftaceus ruficlens; lirel. lis prominentibus triquesris angulofis fimpli. cibusque.

Opegrapha fderella. Svet. Stjernlaf.

Upegrapha rufeicens, Perfoon. Schrad. Icon. Perfoon Ult. Ann。 d. Bot. St, 7. T. 2, f. 3. A. a.

Habitat ad truncos arborum.

55. Lichen afroites - cruftaceus; lirellis applana. natis itcllatim fisfis pedatisque. Opegrapha aftroites. Svet. Strällaf.

Opegrapha radiata Perfoon. Schrad.

Icon. PerfoonUtt. Ann. d. Bot. St. 7. T. 2, f. 3, B.6. Habitat in ramis Fagi, Carpini. Obf. Variat crufta albida es pallide olivacea. 
56. LICHEN macrocarpus - cruftaceus albus; lirellis longisimis parallelis apice furcatis. Opegrapha macrodarpa. Swet. Siroklaf.

Opegraphis macrocarpl. Perfoon, Schrad. Icon. Perjeon Ut. Amal. d. Eoto St. 7. T. I. f. $\mathrm{I}, \mathrm{a}, \mathrm{b}$,

Habirat ad ramos Corgli avellana allarumque arborum.

57. Lrchen frribtus - cruftacens albo pallefcens; Jirell is immerfis pulverulentis ramolis, ramis divaricatis.

Opegraph fcripta. Svet. Skrifulaf.

Lichen fcriptus. Linn. Retz. Pr. 2, Liljshl. Hoffb. Gung. Scop. Crantz. Reich. Fl, Neck. Weis. Schreh, Pollloch. Web, Hudf. Lightfo Hoffm. En. Leers. Alloon. Scholl. Granel sylt. Muller.Roth. Tertit. LumnizzSchrank. Wuther.

Opegrapha pulverulenta Perjoon Uit. Ann. d. Bot. St. 7.

Verrucaria fcripta Wigg. Prim.

Icon. Perfoan 1. c. T. 1. F, 2. B. b. Hoffmo Enum. Lich, T. 3. f, 2. a. b. Dillen. Hitt. Mulc. T. I8. f. I. Michel. Gen. T. 56. f. 3. Habitat in cortice arborum juniarum.

58. LICHEN litterellus - cruftaceus candidus; lirellis radiato ramolisilimis cxíio nigris. Opegrapha litterella. Svet. Chineslaf. Habitat in cortice antigia Populi tremulx. Obf. Pulchra Jpecies: Lirellarum figura, litteras Sinenfum grodanmodo referente; a proori latis dïfinglus videtur.

59. LICHEN Serpentinus - cruftaceus lacteo cyanefcens; lirellis confertis flexuolis limplicibus ramolisque creliis,

B 5

Ha: 
Opegraph forpontina. Svet, Slimgerlafs

Habitat in toceis, Populi nemulic \& Tilia ato nole contice.

Obl. Crupe color margaripactas. Livelle plurime

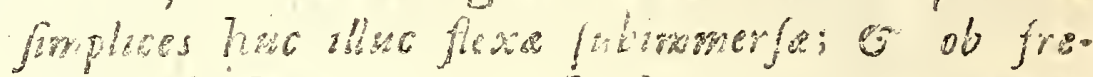

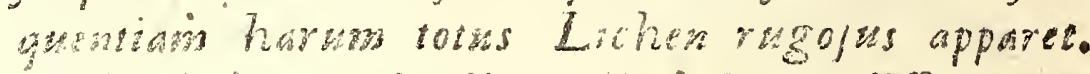

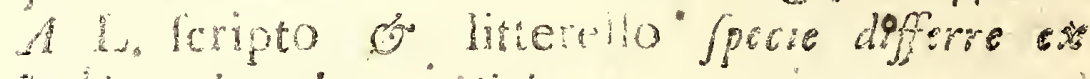
- habis at coloreconicin so

+ I. pracinetus crupacens olvaceus nitidus, lincolis ngits limiang; lirellis glabris ra. molis thelicibugue. - Opegrapha bimata Perfoom. UR. Ann d. Bot. St. 7. Opegraptio pracinds. ram, arb.

+ L. ecraj cruftacens albus glaber rubugo. fus; linellis fublexuofs glabris mplicibus ramofisque, ranis genkulais parallelis. $\rightarrow$ Opegrapha cerali Perfogn Unt. Ann. d. Bot. St. it.

Opegrapha cerafo. cort, Iruni avium anuor.

NoTA. Non dubio fore, gati judicene, inter jam enese

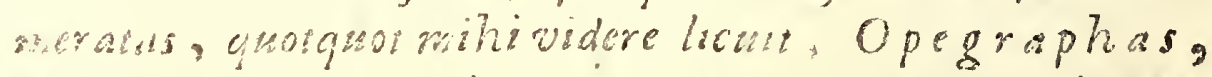

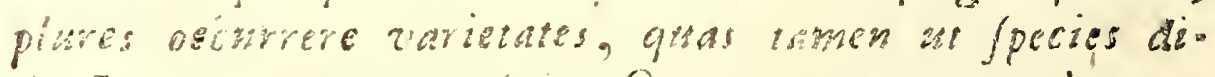

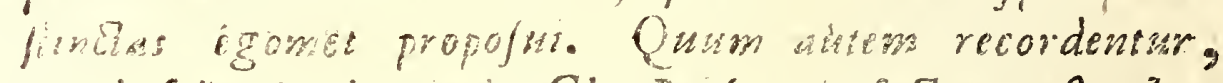

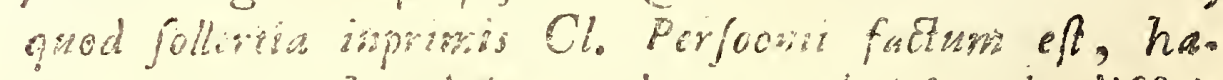

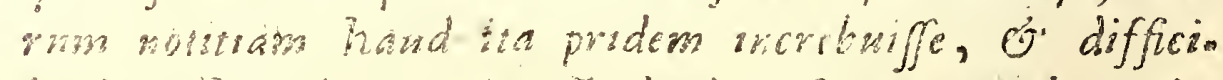

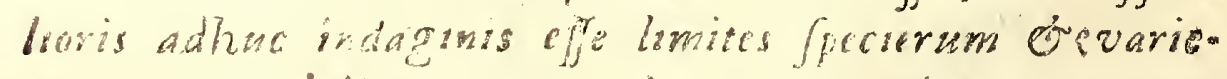

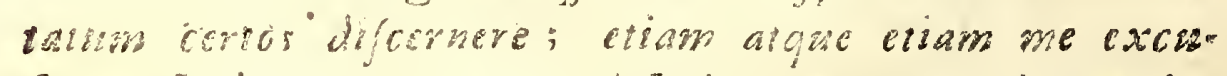
fatum habeani fpero. quod heso memoratas in emolu.

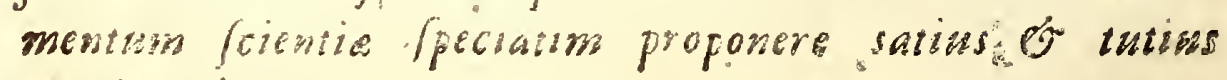
ornsino dwerims. 


\section{$-2 \% \cdot-$ \\ Tribus 4.}

Variolaria, Svet. Korplatyaro

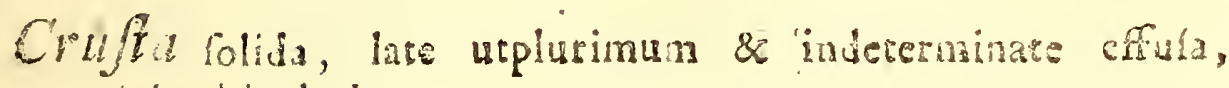
Iuborbicularis.

Receptaculia: Glomeruli figperficiales farinofi convexiu. fculi vel pulvere farfat hoc elabence parum excavati, Icutelliformes.

60. Lichen faginens - cruftaceus rugofus orbicula ris cineralceas; glomerulis convexis albidis. Variolaria faginea. Soret. Boklaf.

Lichen fagineus Lina. Retz, Pr, 2. Liljebl. Hudif. Lightf. Weis. Neck. Delic, Leers. Leyss, Pollich. Hag. Scop. Reich. Fl. Haffn。 Enum. Schreb. Spicil, Gunn. Allion, Maller. Scholl. Roih. Tent, Gisel. Syit, Wither. Lumaitz, Schrans.

Variolaria faginea Perfom Uta, Ann, d.Bot, St.7. Verucaria faginea Hoffm. Deut. Fl.

Verrucaria unerculola Hoffm. Dent. Fl,

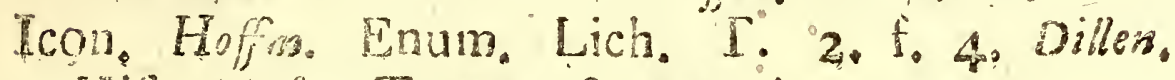
Hit. Mafe, T. 18 . f. 17. A.

Habitat in cortice arborum, precipte Fagi', $T$. live, Carpini \& Eraxini.

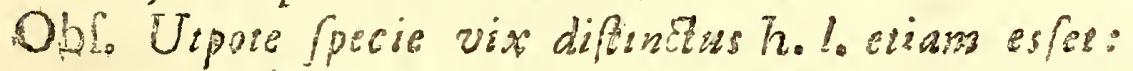

Lichen earpineus Linn? of Auclornm? Sod fyo nonyma dubira atque yatis indererromara. Ne differs:

Lichen orbiculatus Schreb. Spicil. quake:

Verrucaria orbiculata Hoffm. Deut F\%. Ejusd. Enum. Lich. T. 7. fo 2. nifs crupa liseis car.

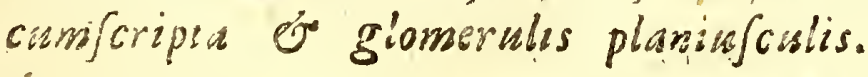

61. Lichen lastezs - crultaceus fubrimofus albidus; glomerulis hemisphericis candidis, Vidroularia labere Svet, Mjólklaf. Li: 


\section{- 28. -}

Iichen Bąteus Linn? \& Auctorum?

Lichnocandidus Hoffm. Enum, Lich.

lichen albus Roth. Tent.

Variolaria lackea Perfoon. Uft. Ann.d. But. St.7.

Pfora lactea Hoffm. Deut: Fl.

Icon. Hoffm. En, Lich. T. 4. f. 6 .

Habitat in faxis \& rupibus.

Obf. Etiam foutelleferam esle hanc Jpecism, foutellisque atris rarisfrme pradicam polulat Kroffm. Dext. Fi. 20 pi 167.

An hic, vel L. impolitus, verus fit L. la. cteus Linn. hasd cerlo conflai.

An varietas L. Faginei a loco tanum dependens?

62. LICHEN aspergilins - cruftaceus rimofus effufus cinereus; glonerulis planiufculis obloletis in. canis.

Variolaria afpergilla. Svet. Berglaf.

Variolaria pallida: Perfoon Uit. Annal. d. Bot. St. 7.

Habitat in rupibus \& faxis.

63. Lichen difcoiáens - cruftaceus rugofus albus; glomerulis polt lapfum farinæ concavis diftormibus concoloribus.

$V$ ariolaria difcoidea. Svet. Ruflaf.

Lichen faginei var. Auctorum.

Lichen albefcens Hudf. Fl. Angl. Gmel, Sylt. Linn.

Lichen fcaber Scopoli Carn. 2.

Variolaria difcoidea Perfoon Uft. Ann。St. 7 .

Verrucaria faginea W.gg. Prim.

Verrucaria ciilcoidea Hoffm. Deut, Fl.

Verrucaria nodulóa Hoffon. Deut. Fl. Icon. Hoffm. Enum. Lich. T. 7. f. 5. Dillen. Hilt, Nufc. T. 18. f, Ir. Michel.Gen. T.53. f. I, Habitat ad truncos arborum vetuftos, 
64. Lichen dealbus - cruftaceus rimofus albus; glomerulis papilleformibus approximatis $\mathrm{gra}$ nulofis concoloribus.

Wariolaria dealbava. Svet. Kritiellaf.

Habitat in rupibus.

Obf. Solus lategue expanfus vel etiasn crim I. corallino immixeus occurrite. Vix hujus varietas out differentia fexus. Crufra crafsa tota glome. rulis papillofis tegitur, deterfa farina fupra pla. niufculis ofo pallide intefcentibus.

65. Lichen amphibolus - cruftaceus tenuis albocinerafcens; glomerulis 'fparfis minutis fubglo. bofis, poft lapfum farinæ fcutelliformibus nigris. Variolaria amphibola. Svet. Tudtydslaf. Habitat in cortice vetufta Quercus rob. Obf. Singularis cerie. (pecies ob duplicem Receptaculorum faciem. Prisso enim fubglobola funt of undique pulvere coharente albo, tegd, hoc vero ablato corpus cylindraceum apparet l. Subrotumdum atrum, poro apice periufusn, quod autem per atatem ampliatum formam foutella marginata asfumit, difco concavo, alque etiam in eo ftats pralvere repleze ac Juffula.

+ L. viridulus . - cruftaceus farinofus cinereo virefcens; glonerulis hemifpharicis viridulis. - Lichen viridulus Hoffm. En, Lich, T, 2. f. 6. Gmel. Syft. Linn.

Variolaria viredula. lign. putr. 


\section{$-30$. \\ Tribus 5.}

UlRCEOLARIA. Suet. GROPLAFVAR。

Cruffa colida crasfuccula rimolo areolata, (in arborum cortice tenuior contigua) aut verricofa, fuborbicularis, Receptacula: Scutelle crufx verrucis a areolis immerfx, urceolatx.

66. Lichen? lepedinus - cruftaceus glaucovirefcens lavis contiguus; verrucis Iparlis glabris coa? coloribus pertulo urceolatis.

Urceolaria? lepadina. Svet. Opperalaf.

Habitat ad corticem Ulmi.

Obf. Crufa contigua o guafi membrainacea. Ver. ruce Sparse hemispharice vel fubconica apice fces. tellifera, foutellarum fisndo ampliori nigricante. ore aperto Juperne magis coarciato, margine gla. bro iniegro. Habitus omnino Is. pertuli. Species fingularis ejusdem forte generis cum L. pertulo, of hymenio, Ob virrucas concavas femper a. perias inter Verrucarias locum habere vix po. tefa, fed Jrceolarbis poitus affociandus.

67. Lichen agelens - cruftacens rimofus levigatus albicans; verrucis parlis planinlculis feutelo Jiferis, fcutellis confertis immeris nigris cæefiisque.

Urceolaria agclea. Svet. Blisdlaf.

Habitat in cortice populi tremulz.

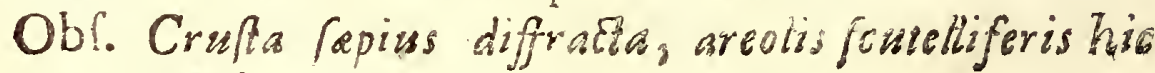
illic elabenitibis.

58. Lichen gibbofus - cruftaceus varrucolus; verrucis fubglobofis albocinerafcentibus glabris fcutelliferis; fcutellis fubcontiguis impreslis concavis nigris, margine rugolo, Ur- 
Urecolaria gibbora. Svet. Snörplaf. Lichen gibbofus Dick/. Crypt Br. Wither, Arr. Gmel. Sytt. Limo.

Lichen ocellatus? Bellardi. Villars. Icon, Dickf. 1, c. 2. T. 6. 1. 5o Villars Fl。 Delph. 1. 5i?

Habitat ad rupes \& faxa.

Obf. Quenadmonm in L cineren, exiam in hoc foutelle fepiats pistes in endem verraca occurrumb at oftoio quafic contrailo dewum hiaries, five margine more fphincteris rugofo tumidislo cincte. Crufta, preter verrucas instio planizficulas, vis ulla, fod interftuia more L. Hoffmanni plerum. que nigra; Colore verrucarno of Scutellarm, earumgin forme sb eo difingess.

69. LicheN Hoffuani $\rightarrow$ cruftaccus vermcolus; verucis margine depresfis fubcontortis papil. leformibus plumbeis apice fcutelliferis; fcu. tellis lubfulcis inipresís concavis, margine rugolo pulverulento albo.

Urceolaria Hoffmanni. Sveto Hoffranslar.

Lichen rupicola Hoffm. Enum. Lich, Gmel. Sylt. Linn. Wither. Arr.

Verrucsia contorta Hoffm, P1. Iich, Ejuscdo Deut. Fl.

Icon. Hoffns. Pl. Lich, T. 22. I. I - 4. Ejusd. En. Lich. T. 6. f. 3.

Habitat ad faxa \& rupes fchiftofas.

Obf. Singalis verrucis rarius plus quam una fcro tella impolua, ore of margine, us in L. gibbo. 10, contrabla, per atasem ramen magis explansta dilataia. Verrice interdum /piraliter gues. A contoria, margine depresfori fimplici duplicive, diffinkte, of fere absque crufta, hic illic Jparfa, Solutarie, rarius confluentes. Color jam albido cinereus, jam cirerco plunbeus. Li. 
Lichen rupicola Linn. Lightf, \& Religuorum neque ohusc neque L. gibbolum Jpectat.

70. Lichen forupofas - crufraceus granulatus albocineralcens ;ofutellis fparlis urcenlatis inmerfis nigris, margine crenulato turgido inyoluto. Urceolarsa forupola o.svet. Kuplaf.

Lichen fcrupolus Schreb Spicil. Rezz. Pr. 2. - Liljebl. Hoffm. Linuin. Dicks. Gmel. Sylt. Linn. Schrad. Rath. Tent. Sibih. Oxon. Vallars. Bellardi. Wither.

Lichen cxcavatus Relhan. Cantab.

IJichen ocellatus Wriff. Schr. Nat. Fr. Gmel。 Syft. Linn:

Lichen pertufus tulf. ap. Jacqu. Coll. 2. Pateliaria fcrupoía Hoffm. P1. Lich. Verucaria fcrupola Hoffm. Deut. Fl.

Icon, Hoffin. Pl. Lich. T. 11. . 2. Ejusd. Enum,

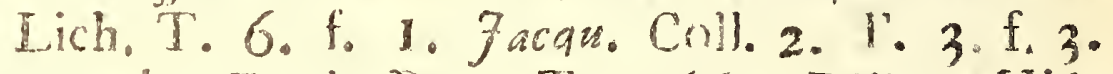
Soverby Engl. Eor. T. 266. Dillen. Hit. Mufc. T. 18. f. is P. Muchel. Cent T. 52. f. 6. Haller. Enum. T. 2. f. 6. Ejusd. Hiit. 47.1 .6 .

Habieat in faxis \& rupibus.

Obl. Variat magnitudine, crufta aquabili., Jubri. mola of alba, gránulata; colore cinereo, albo, fubcafso algue pullida, nee non foutellarum fure zmmerfo l. magis inperficiali, harumque margio ie vix ullo.

71. LICHEN cinerezs - cruftaceus verrucofo - areolatus rimoius cinereus nigro limitatus; fcutellis impresfis urceolatis nigris.

Urcoolarin cinerea. Svet. Alkgrälaf.

Lichen cinereus Linn. Liljebl. Hoffm. Enum. Lich.

Icon. Hoffm. Enum. Lich. T. I4: 3. Habitat ad lapides. 
Obr. In fingula areola. foutella incordum 20 30 msdulantur of per atatem majis elevantsar Cras fte margines plerumque linea atra limita ?.

Lichen. cinercus Aucterum vix genanus Lino nat, Sed pouzus L. whephromelus. ito

72. Lichen tesfulatis - crultaceus aguabilis tenuis ine rimolus albicans; fcuterhs impresîs nigris.

Urcoolaria tesfralata. Svet. Rämnelaf.

Lichen punctatus Duckf.Crypt. Britt.Retz.Pr. I. Verrucaria teslelata. Hoffm. Deut, H.

Habitat in rupibus \& faxis.

Obl. Scretle primo punttiformes, impresfa, atate muleo majores, una cum areolis emergunt of a crufta marginate obveniuni. A Lo cinereo of rimolo utique differt.

73. Lichen Acharii - crufaceus levigatus fubrimofus pallido teltacens; fcutellis immerlis urceolatis rubris.

Urceolaria Acharii. Svet. Achariila?. Lichen Acharii Wefring. Collect. Icon. ad Titulum prrefentis opufculi. Habitat in rupibus lupra granik. ad ripas Sinus Botbnici.

74. LICHEN geographicus - cruftaceus rimofus flavovirelcens, margine areolisque quibusdam inmixtis, fcutelliferis nigris.

Urceolaria geographica. Svet. Chartelaf.

Lichen geographicus Linn. Retz. Pro 2. Liljebl. Hoffb. Gunn. Leers. Hudr. Lighef. Leys/. Pollich. Schreb. Soic. Weis。 Weber. Neck. Hag. Matus cka. Hoffm. Enum. Gmel. Sylt. Linn. Crantz. Allioni. Doerr. Mull. Mohr. Schiolto Roth. Tent, Reich. El,Wither. 
Lichen atrovirers Linn. Retz, Pr. 2. Liljebl, Hoffb. Hudf. Lightf. Gmel. Sylt. Linn. Iichen Oederi, Gunn. Retz. Pr. 2.

Verrucaria geographica Hoffm. Fl, Lich, Ejusd. Deut. Ft.

- Verrucaria atrovirens Hoffm. Pl. Lich. \& Deut. Fl,

Icon. Eoffm. Enum. T. 3. f. I. Ejusd. P1. Lich. T. 17. f. 4. T. 54. f. 2. Sowerby Engl. Bot. T. 245. F\%.Dan. T. 468. f. T. T. 472. f. 3. Dillen. Hifto Mufc, T. 18. f. 5. Michel. Gen. T. 54 . f. 7.

Habitat in rupibus \& faxis quartzofis.

Obf. Pro diversa alare forma variat atgue colore, demum ofufceforite, cintreo of prorfus nigro. Cruffe bafis plerumgue atra.. Punctis \& lineis nigris haut ravo percussus reperitur. Scutelle arre excavata fubputverulesta. L. atrovirens juniorem; L. geographicus maturum; L. Oederi Gunn. Senefcentem jom Lichenem exhibet.

t L. Wutfeniz - crunaccus rofeo.purpurafcens, fcutellis impresfis planiuficulis nigris. - Lichen namoreus Wrulf. ap. Jacqu, Coll, 2. T. I3. 1. 1. Retz, Pr. 2. - Lichen marmoratus Gmel. Sylt. Linn. - Vertucaria purpuralcens Hoffm. P1. Lich. T. I5. f. I. Ejusd. Deut. Fl.

Obf. Crufta interdum rivulis ferpentinis decusfata \& ambitu nigris, Scutellæque pulverulentre atro fanguinex.

Urccolaria Wuilfenii. faxa.

* I. cinereocafírs - cruftaceus albo cinerafcens; fcutellis inmerfis urccolatis cæxilis, margine cremulatis - Lichen cinere ocafius $S w$ artz. ined. c. Ic, Urceolstia sinereocafa. Terra Ind. Occ. t L. 
† L. exanthematicus - cruitaceus cinereus; fcu. tellis minutisinis carneis claufis, demum expan. tis concavis, immerlis in crufte foveolis aibis. - Lich ex exanthematicus Smith. Act. Soc. Linn. I. T. 4. f. I. Dicks. Bellardi - Lichen clau. fus Hoffm. Enum, Lich. Grael. Syit. Linn, Lichen volvatus Villars Fl. Delph. T. 55. Verrucaria claufa. Hoffm. Deut. Fl. Urceolaria?'exanthematica. rup, calc.

* I. excavatus - cruftaceis pulverulentus rimo. fus cinereo albidus; fcutellis minutis immerfis urceolatis atris marginatis. - Lichen excava. tus Thunberg ined.
Urceolaria excavata.
C. b. fp. argill, aren。

t I. multipunetatus - crultaceus cinereoladeus xquabilis rimofo tesfulatus; fcutellis impresis contiguis demum marginatis. - Verrucaria multipuncta Hoffm. Deut. Fl.

Urceolaria multipunciata. fax.

t L polygonins - cruftaceus cinereus tenuis areolis tesfulatis poro uno tribusve notatis - Lichen poly. gnnius Villars Fl. Delph. T. 55. Bellardi App. Fl. Ped.

Urceolaria? polygonia. lap.

Nora: Lichen excavatus Hoffn. Enum. Licha T.7. f. 4. Gmel. Sylt. Linin. \& Patellaria excavata Hoffm. Pl. Lich. I. T. 23. f. 3. non verus eft $L i$. chen led fungorum affinis, nempe Spherobolus ro. faceus Tode Fung. T. 7. f. 57. Gmel, Syf. Linno 


\section{$-36 .-$ \\ Tribus 6.}

\section{Patellaria Svet. Plättlafyar.}

Crufta folida contigua \& a aquabilis aut rimora areolata etiam granulata, luborbicularis vel irregulariter expanfa。 Receptacula: Scutelle fesfiles, fuperfiriales, concavx, plana \& convexiufculix marginate, vel eriam hemisphxricx \& fubg!obofx inmarginata feu margine tenui \& vix manifelto ad bafin cinctx.

* I. Scutellis albidis, pallidis $\mathcal{S}^{\circ}$ teftaceis.

75. LICHEN parellus - cruftaceus rugofo verrucofus rimuilis albus; fcutellis concoloribus, margine turgido.

Paecllaria parella. Svet. Krieftfenslaf.

Lichen parellus Linn. Retz. Pr. 2. Liljebl. Hoffm. Enum. Lich. Huds. Web. Leightf. Neck. Leers. Doerr. Roth. Tent. Mohr. Bellardi. Wither. Relh. Gmel. Sytt. Linn. Verrucaria parella Wigg. Hoffm. Deut. Fl. Patellaria perella Hoffm. Pl: Lich. Icon. Hoffm. Pl Lich, T. 6. f. 2. Dillen. Hitt. Mufc. T. I8. f. 10.

Habitat in muris \& rupibus maritimis prefertim calcareis.

Obl. Per atatems color in cinereo pallefcentem abit or ob musuam presionem fourella japous angulo. Jet evadunt.

76. LICHen pallefcens - cruftaceus verrucofo granulatus albicans; fcutellis pallefcentibus, margine t turgido albo.

Patellaria pallefcens. Svet. Bleklaf.

Lichen pallefcens Linn, Liijebl. Hoffb. Pfora alabaltrina: Hoffm, Deut. Fl. 
Habitat ad truncos arborum.

Obf. Hic, qui verus eft L. pallefcens Linnai,exe. teris prorfus ignoous videtur, faltem fynonyma Auitorum adeo incerta, ut nulla allegare poinerim. Varia enim species fub hoc nurmine upud cos occurrunt.

In juniori Lichene Toutellarum difcus dilute flavefcens, in fpeciminibus vero vetufteribus totus Lichen concolor fordide e cinereo pallefcens. Lo. co \& foucellsrum juniarum colore, crufta contigua verrucofogranulata a L. parelio quidem differt; an etram (pecis?

Forte a noflo difinguendus eft:

Lichen pallelcens Widfo ap Facgr. Coll. 3. T. S.

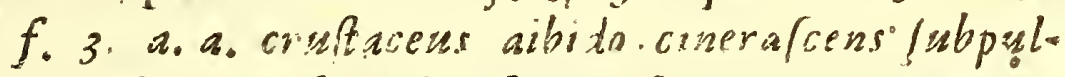
veralentus: foutellis fitipatisfome aggregatis ci. nereis, margine albodrori.

77. Lichen upfalienfis - cruftaceus tenuisfumus la. vis lubpulverulentus alho glaucefcens, fcu-. tellis planis pallidis, margine turgido.

Patellaria upfalienfis. Svet, Upfalalif.

Lichen upialınfis. Linn. Rete. Pr. 2. Liljebl.

Hoffb. Gun\%, Dicks. Hoffm, En, Lich, Wi. ther. Gmel. Sylt. Linn.

Patellaria upfalientis Hoffin. Pl. Lich.

Pfora up alientis Hoffm. Deut. Fl.

Icon. Hoffin. Pl. Lich. T. 2 I. f. 2. Ejusd, Enum. Lich. T. 7. f. I. Dickf, Cr, Britt. T. 2. f. 7. mala.

Habitat fupra mufcos locis campeftribus arenofis elatis.

78. Lichen tartarens - cruftaceus granulatus albo. virefcens; fcutellis teltaceis demum convexis rugofis, margine tumente albo.

Patellaria tartarea. Sveto Byttelelaf.

Lichen 


\section{$-38 .-$}

Lichen tartareus Linn。Retz. Pr. Liljebl. Hoff'b. Huds. Lightf. Pollich. Hag. Gunn. Leers.

- Roth. Tent. Murray Prodr. Hoffm. Enum. Mattufe. Vulf. Allion. Wither, Lumnirz.Gmel. Sylt. Linn.

Lichen laxorun Fl. Dar, Retz, Pro 2.

Lichen androgynus Hoffm, En. Lich, Roth. Tent. Gmel. Syit, Linn.

Lichen frigidus Linn. Swurtz, Liljebl: Retz。 'Pr. 2i, Hoffb. Hoffm. Enum. Dicks. Wither. Ehrh. Achar. Act. Svec, V. IS.

Plora frigida Hoffm. Deut. Fl.

Verrucaria tartarea. Wigs. Hoffm. Deut, Fl. Icon. Facqu. Coll. 4. T. 8. f. 2, Fl. Dan. T. 7 2. f. I. Sowerby Eagl. Bot. 2. T. Is6. Hoffon. En, Lich. T. 7. f. 3. Dillen. Hit. Mulc. T. 18. f. I3. (Swartz. Meth. Mulc, T. 2. f. 4. AR. Med. Svec. T. I5. f. 4. Achas. Nov. Act. Sc. Svec, Vol. 15. T. 6. f. 2. L. frigidus ).

Habitat in rupibus \& fupra terram locis fteri. libus fupra mufcos.

Obf. Crupa foinulofa ac ramulofa L. frigidi in alpibus lecti a fubftratis mufcoram larculis of folits originem ducit, ut in L. uplalienfi eicam contingit. Scutell trum color in L. fri jido vio vider in carnearm inclinato

79. LICHEN orofhens - cruftaceus pulverulentus rimofus tenuis fulphureus; fcutellis convexis pallide lutefcentibus.

Patellaria orofthea. Svet. Hallelaf.

Lepra fulphurea? Ehrh, crypt.

Habitat in faxis \& rupibus.

Obf Late dispersa crufta, Li hrematommx junioris frrillima, rarisfome profert fcutellas hernispharicas, vix marginatas, grasis pruinofas, mi. 


\section{- 39. -}

nuras, crufa fubconcolores, os hinc primo intui. the vix difcernendas. Cum L. lutefcente, zn cortice tantum proveniente, minims confundendus. v. Jupra t.s.

* 2. Scutellis flavefcertibus.

80. LrCHEN Ehrhamianus - cruftaceus rugofo gra- nulatus albovirefcens; fcutellis marginatis demum convexis flexuolis difformibus conglo. batis flavefcentibus.

Paidlariaz Ehrhartiana. Svet. Ehrhartslaf.

Icon. Tab. noftra 2. f. 1 .

Mabitat in cortice Quercus, Tilia \& ad ligna fubputrefcentia.

Obl. In quamplurimis crafta maru io perfecta granis feu verruculis obfervantur tubercula alia nigra rugofa fuperimpofua.

Lichen graniformis Hag. Hift. Lich. Gmel. Syfo Lisn. forte hujus crufte snitium, faltem charalter defcriptio of figura optima quadrant; nam pro diverfa Lichenis nofri atate diverfam induit firulturam atque coiorim crufe.

8I. TICHEN lucidus - cruftaceus pulverulentus viridi flavefcens, fcutellis minutis convexis flavisfimic.

Patellaria Incida. Svet, Lyslaf.

Habitat ad rupes.

Obr. $A$ L. flavo, cui proximus, colore \& loco diverges.

* 3. Scrtollis lntefcentious EO fulvis.

82. LiCHEN epanorus - cruftaceus pulverulento granulatus viridifulvus; fcutellis concoloribus, margine convexo pallidiore. Patellaria epanora. Svet. Gruslaf. Habitat in faxis \& rupibus. 
Obf. Scutclle plerumgue congefte, ob mounam pres. fronem formam non varo prabent irregularem, at numgiram convexas invens, nec margine crente latas; Quo nota nt elsum culore, loco fo toto hat buru a L. Vario diferepar.

83. LICHEN varizs - cruftaceus tenuis; fcutellis aggregatis luteis varisque, margine pallidio. re urmum flexuolo involuto crenato. Patellaria varia. Svet. Trimelaf.

Lichen varus Ehrh. Crypt. Exf. Reiz. Pr. 2. Lichen pallecels Schrank. Fl. Bavar.

Patellaria varia Hoffm. Pl. Lich.

Verrucaria varia Hoffm. Deut. Fl. Icon. Hoffrn. M1. Eich. T. 23: f. 4. Habitat ad asferes. \& parietes ligneos antiquos. Obf. Crufa magis perfecta luteovirefcens, fuborbi. cularis, tenursime granulata, fcutellis conflanter concoloribus in cortice Pini fyiveftris occurrit? an. difincia fpecies?

Varictas, crufa tensisfima alba eiv cinerafcente. Scursilbsque perexiguis palludes, jubfufcis of nia gricanibus, margine senussme crenulato albicanre, "in ligno truncorus" denvidato una. cum $L$. byloico, obvia, atatem Lachenus nofiri infantilew indigital of forte eadem ac $\mathrm{L}_{\text {, ninutisfimus }}$ Hag. vide infra.

Color dific frutllarum in hac fpecie admodum variat nempe luteus, pallidus, frocejcens of nio gricans.

84. LICHEN cerinus - cruftaceus cineraficens tenuis; fcutellis luteis, margine albó demum nigrim - canre fubinflexo:

Patellaria rerina. Svet. Vaxgullaf.

Lichen cerinus Ehrh. Lir. exl. Hedwo. Dickf. Schrad. Spicil, Gmel. Sylt. Linn. 


\section{- 4I. -}

Patellaria cirena Hoffm. P1. Lich.

Verrucaria cerina Hoffm. Deut. Fl.

Icon. Hedvig Crypt; T, 2I. f. B. Hoffm. PI, Lich. T. 33. t. I.

Habitat ad aiborum corticen \& epidermidem; in terra fubftratis mufcis putrefactis, faxisque. Obf. Variat crufta alba ej nigra (parafittca) vel prorfus mulla, foucellas diverfe magnitidinis of coloris, wit in:

Verrucaria gilva Hoffm. Deset. Fl. fcuellis for. dide cerinis cinereo marginatis.

Verrucaria aureocerina Ejusd. lo co Scutellis rufefcenti cerinis demum convexiufoulis, margine albo.

Verrucaria ravida Ejusd. l. c. Scutellis confers tis zsmbrino fufcis luteis crufas cinerea, margine concolore.

Lichen ftillicidiorum? Fl. Dan. T. 1063. f: 2. Retz. Pr. 2. Verrucaria ftillicidiorum Hoffmo Deut. Fl. crufta viridi cinerafcente, Jcurellis cerinis viridantibus margine cinereo. Nec forte differt:

Lichen aurantiacus Wulf. ap. Facgu. Coll.30 nigricans tuberculis globofis faturate aurantiacis.

85. LiCHen vitellinzs - cruftaceus granulofus vitellinus; fcutellis aggregatis demum convexis irregularibus concoloribus.

Patellaria vicellina. Svet. Aggulelaf.

Lichen vitellinus Ehrh, Cr. exf。Retz. $\mathrm{Pr}_{0}$

Gmel. Syft. Linn.

Patellaria vitellina Hoffm. Pl. Lich.

Verrucaria vitellina Hoffrn. Deut. Fl. Icon. Hoffm. Pl. Lich. T. 26. f. 1. T. 2\%. F. 2\% Habitat in Icandulis \& trabibus ligneis lemipu. tridis.

C 5

$\mathrm{Obr}_{\circ}$ 
Obf. Margo fouzellarum aliquando pallidior per atatem diverfo modo fiexns of crenulatus.

Farietates praciptic junt:

Lichen bysinus Hoffm. Enum. Lich. T.4. f。 7. Grael. Syit. Linn.

Verrucaria bysina Boffm. Deut. Fl. cruftani-

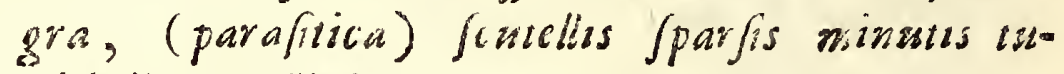
midulis volelimis.

Vernucaria aurella Hoffm. Dewt. Fl. acruflacens, foutellis minutis Jparfis. ad rupes.

Verfucaria arcuata E.jusd. l. c. crufla vitellina, foutellis lividis foralits. Pl. Luh. T. 27. f, 2.

86. Licien luteus - cruffacets pulverulentus cine. seo pallidus; fcutellis planiufculis marginatis luteis.

Petellaric lusea, Svet. Fráknelaf.

Lichen liteus Dicks. Crypt, Britt, I, Gwel. Sylit. Linn.

Icon. Dicklon. 1. c. T. 2. f. 6.

Habitat in terra \& inpra nufcos.

87. IICNeN latcolus - cruftaceus granulofus einereo virefcens; fcutellis fparfis demum convexis totis huteo ferngineis.

Futillaria luteola. Svet. Gulbrunlaf.

Lichen luteoius Schrad. Spicil. Fl. Germ.

Liclen corticalis Rutfiom. Disf.

Lichen lutereus Gmel. Syft, Linn.

Lichen vernalis Hoffm. Enum, Lich, Icon. Foffm. En, Lich. T. 5. f. 1. Habitat in cortice Salicis, Aceris.

Obf. Varictas vel potius infans hajus eft:

Lichen rubellus Ehrh. Crypt. exf. qui idem ac: Verrucaiia rubella Hoffm. Deut. Flo crufta albido flavefcerte cincrea, foutellis rufefcentubus, margere pallidiore.

Occurs 
Occurrit etian foutellis futco rubellis planitufculis, margine concolore, cui nomen efl:

- Verrucaria fuicorubella Hoffm. Demo. Fl. Nec Ypecie differt:

Verrucaria vernalis Ejusd. l. co crufla flavan fcente, foutellis rufefcentibus convixis margia ne difco concolore.

88. Lichen rupefiris - cruftaceus tenuisimus cinereo fufcus; fcutellis hemisphericis immarginatis lateis rutisque.

Paiellaria rupeftris. Svet. Stenlaf.

Lichen rupeftris Scop. Carn. 2. var. a $B W e b$. Spicil. Roth. Tent, var. A, B, Hojfm. - Enum. Lich.

Lichen rufefcens Retz, Pr. 2, Bellardi. Gmel. Sylt, Linn.

Verrucaria rufelcens Hoffm. Pl. Lich, \& Ejusd, Deut. Fl.

Icon, Hoffm. Pl. Lich. T. I7. f. I.

Habitat in faxis \& rupibus.

OjS. Variat fcutellis luteis of rufelcentibus, jam depressis; cruiba obfcura of olivaceo flavefcente, aut interium plane defciente.

* 4. Scutellis aurantiacis.

89. LiCHEN erythrellus - cruftaceus areolato fubragulofus lrevis tenuis flavislimas; fcutellis demum globolis totis rubroaurantiacis nitidis.

Patellaria erythrella. Svet. Gyllenlaf.

Habitat in durisimo granitide.

Obl. Cruta late effufa fuborbicularis, ad parieles rupiun verticales tenacisfrme afixi, infeparablis. Scutella juniores planiufcula flavo frobmarginate.

90. Lichen falicinus - cruftaceus verrucofo granulitus fordide flavas; fcutellis aurantiis demu n convexis; maryine pallido.

$p_{d *}$ 
Patellarial falicina. Svet. Pihllaf.

Lichen lalicinus Schrad. Spicil. Gmel. Syft, Linn.

Verrucaria falicina Hoffm. Deut. Fl.

Habieat in cortice Stlicis, Ulmi.

ObP. Crupa flavicante a Lo aurantiaco differt, fo. mallima L. lutefcentis, at, lente obfervata, diveriossema.

91. LJCHEN aurantiacus - cruftaceus albocinerafcens nigro linitatus; fcutellis aurantiis demum convexis, margine flavo.

Patellaria aurartiace. Svet. Pomerantslaf.

Lichen auranciacus Lightf. Retz. Pr. 2. Liljebl. Hoffm. Enum, Vahl Fl. Dan. Wild. Prodr. Roth. Tent. Lumenisz. Bellardi, Gmel. Syft, Linu.

Lichen flavorubefcens Huds, Oed. Relh. Wri. ther. Gmel. Sylt. Linn.

Verrucaria aurantiaca. Hoffm. Deut, Fl. Icon. El. Dan. T. 955. t. 1. mala. Habitat in cortice arborum prefertin ad caud, Pop. trem.

Obf. Crufa orbicularis of quavdo alba atgue ints. gra efl linea nigra circumforipta.

* 5. Scutollis rubris E ferregincis.

92. LICHEN cinereofufcus - cruftaceus tenuis albocinereus; fcutellis planis rufofurcis demum convexis ccntluentibus, margine tenui con. colore.

Pateliaria cinereofufca. Svet, Gräbrunlaf. Lichen cinereofufcus Weber. Allion. Schrad: Spicil. Rosh. Tent. Gwel. Sylt. Linn. Lichen ferrugineus Hudion. Fl. Angl. Iichen ferruginofus Gmei. Sylt. Linn. Lichen vernalis Lighef. Fl. Scot. Wither? Patellaria cinereofulca Hoffmo, PI, Lich. 
Patellaria ferruginea Hoffm. Pl. Lich. Verrucaria cinereofufca Wigg. Primit. Verrucaria ferruginea Hoffim. Deut. Fl: Icon. Hoffm. Pl. Lich. T. 12, f, I, T, 350 f. 1. Dill. T. 18. f. 4 ?

Habitat ad Fagi, Tilix, Salicumque cortice. Obf, Margo fcuellarum tenursfrusus concolor per a. tatem ornnino evanefcit.

93. Lichen cafiorufus - cruftaceus rimofus cinerea. cæíus; fcutellis planis rufis demum conve. xis, margine tandem flexuofo crenato conco. lore.

Patellaria cafiorufa. Svet. Gräródiaf.

Lichen cæliorufus Schrad, Spicil. Fl. Germ.

Habitat ad faxa duriora.

Obf. Scutella in infantia parza, plane, margine, in etate, maxime provedirs Speciminibus, femper concolore of hinc a sequenti omnino difinctum puto.

Differt a L. cinereo fufco, loco, crufta for. ma o colore, nec non Jcurcllis marginatis to faturatius coloratis.

94. Lichen crafpedius - cruftaceus rimofus albidom crlius; fcutellis rufefcenti ferrugineis demum convexis, margine flexuofo albefcente. Patellaria crafpedia. Svet, Briddiaf.

Lichen arenarius $\operatorname{Per} r o o n$. Uft. Ann. d. Bot. St. $y_{0}$ Verrucaria arenaria Hoffm. Deut. Fl.

Verrucaria cæíorufa Hoffm. Deut, Fl。

Habitat ad faxa arenaria.

Obf, Margines Foutellarum albidi hunc a priori fai is diffingunn; etiam ab infantia pallefcentes.

95. LICHEN hamatomma - cruftaceus pulverulentus viridi fulphureus; fcutellis immerfis demum convexis difformibus ruberrimis. 
Patellaria hamatomma. Svet. Rödplattslaf.

Lichen hxinatomma. Ehrhart. Retz. Pr, 2. Liljebl.Schrad.Spicil. Perfoon. Gmel.Sylt.Linn. Lichen ochroleucus Neck. Meth, Van Geuns. Hoffm. Enum.

Lichen ventofus Pollich. Pl. Pal.

Lichen flaveolus Gmel. Syit. Linn.

Lichen coccineus Dicks. Perfoon. Wither. So: werby. Gmel. Sytt. Linn.

Verrucaria hæmatomma Hiffm, Pl. Lich, \& Deut. Fl.

Icon. Hoffm. Pl. Lich. T. II, f. I. T. 40 , f. I. Ejusd. Enum. T. 6. f. 5. 6. (Dickf. Cr. Britt. I. T. 2. fo I. 3. Sowerby Engl, Bot. T. 223. L. coccin.)

Hebitat ad faxa \& parietes rupium inque cortice Quercus rob.

Obf. Varielates hujus pracipue funt:

Lichen porphyrius Perfoon. U/t. Annal. d. Boto St. ul, qui:

Verrucaria porphyria Hoffm。 Deut. Fl. o E Ejusd. $P l_{*}$ Lich. T. slocrufta alba, foutellis marginatis Jaturate fanguineis.

Lichen querneus Dicks. Cr. Britt. 1. T. 2. f. 3. Reth. Cantabr. Wuth.arr. Gmel. Syft. Linn.qui:

Verrucaria quernea Hoffm. Deme. Fl. crufta cinereo Incefcente; foutellis obfoure furfois of nigris.

Verrucaria frondofa Hoffm. Deut.Fl. \& Ejusd. Pl. Lich. T. 49. f. 1. crulta viridi flave. fcente; foutellis coccineis margine crufta concolore farinoso cinctis.

* 6. Scutellis fubfufcis \& fufsco nigricantibus.

96. Lichen ventofus - crultaceus rugofo verrucofus cinereo flavefcens; fcutellis tumidulis rubrofufcis, margine tenui pallido, 
Patellaria ventofa. Svet, Bläslaf.

Lichen ventofus Linn. Retz. Prodr. 2. Liljebl. Hoffb. Lightf. Neck. Oed. Dicks, G\$ows. Roth. Tent. Wither, Gmel. Sylt. Linn. .

Lichen fcopulorum Fl. Dan.

Lichen lepadolemma Ehrh. Cr. Exficc.

Lichen cruentus Web. Hoffm. En. Leysf. Allion, Roth. Tent. Gmel. Sylt. Linn.

Lichen gelidus Hudfon. Fl. Angl.

Lichen fiavefcens Jacqus. Milc, Gmel. Sylt, Linn,

Verrucaria ventofa Hoffm. PJ. Lich.

Verrucaria cruenta Heffm. Deut. Fl.

Icon. Hoffm. Pl. Lich. T. 27. f. I, Web. Spi cil. T. 1. Fl. Dan, T. 7I2. f. 2. FAcqus. Mifc. I. T. 9. $f_{4}$ I. Dillen. Hilt. Mulc. T. 18. f. 14 .

Habitat in faxis \& montibus.

Obf. Variat crufta plus minusve flavicante, pallio da atque demum cinerea of foutellarum margine vix ullo.

97. LicHeN fubfufcus - cruftaceus albocinerafcens; fcutellis tumidulis fubfufcis variisque, margine albo fubintegro.

Patellaria Subfufca. Svet. Bulllaf.

Lichen fubfufcus Linn. Retz. Pr. 2. Liljebl. Scopol. Lightf. Reich. Roth. Tent. Neck. Mohr. Weis. Schreb. Spicil. Pollich. Hag. Grim. Reyg. Wild. Matusc. Hoffm. Enum. Allion. Gunn. Scholl. Mull. Leers, Lum= nitz. Vither. Gmel. Sylt. Linn.

Verrucaria Subfufca. Wigg.: Prim, Hoffm. Deut. Fl.

Icon Hoffm. Enum. Lich. T. 4. f. 3. 4. 5. Di\% lon. Hilt. Mufc. T. 18 f. I6. T. 55. f. 8. Habitat ad arborum cortices \& Saxa,

Obs 
Ob!. Quamplurimis obnoxius of varietations hic Lichen. Forme to coloris diverfitas a loco er state inprimis dependens, anfarm procul dubso prebuis, multas condere !pecies Jpurias. Cruffa jam tenuis, iam crasla, lavis, granulata, aq. gue etiam pulverulenta. Scutellarum initia Jub. globolos, minima, Sparfa, aut conferitsfima, poo ro impresfa; hinc demsum urceolate apparene fortellie, at utterisus dilatate. So expanfe difcums tumidulum immo etiam convexum prabent mar. gine tumente integro, per atasem vero aliguass. ium flexuofo, five creneshs nocato. Color dio fri conflans gruidem, fed in diverfis individuis diverfus obfervarur, pallidus nempe, lividus, rubellus, fufcus, fufco negricans, margi: the tamen femper crufta concolore cinereo $\mathcal{E}^{\circ}$ albido. Tame crulta guam receptacula aliquan. do pruinoja deprehenduntur:

Varietas Junt:

Lichen umbrinus Ehrh. crypt. exf. qui:

Verrucaria umbrinofulca Hoffrm. Deut. Fl. crus fta cinerea, fcutellis umbrino fufcus, margis ne cinerco.

Verrucaria fuica Hoffm. l. c. crufta cinerea, foutellis confertis depres/is nigrofufcis margio ne albo.

Verrucaria argenteofulca. Hoffim. l. c. cruftas albida, fcutellis faturate rufefcenti of nigro fufcis, margine albo.

Verrucaria pallide fufca. Hoffma l. c. crufta albida, Tcutellis pallide rufefcenti fufcis of difcoloribus aemum angulofis, margine albo: Ab hac parum forte differt:

Iichen bysfinus Dicks. Cr. Britt. in quo /crstellarum color variat albus, pallidus, flavefoens of fufcus. Crufta orbrcularis alba, fed 


\section{$=49 .-$}

color niger vere parafricus. Hujus eliam lo. ci fortass eriz:

Lichen tumidulus Perfoon. Uft. Anno do Boto St. 11: qris:

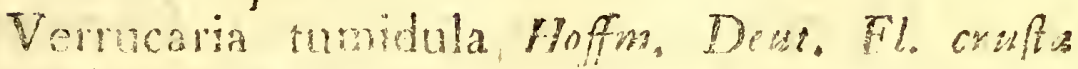

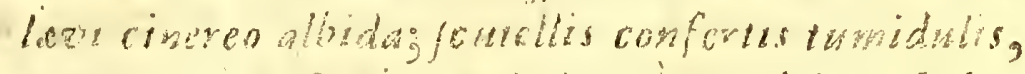

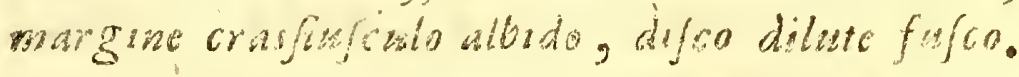

98. LICHEN defperfus - conftacens foutellis minutis diperis fubfulcis, cans uigrisque, margine crenulate allo.

Patellaria difperfa. Svet. Briflet.

Lichen difpertus Perjoon.Ut. Amn.4.Bot. St.7.

Verucaria difperla Hoffm. Deut. El.

Habitat ad lapides calcarios.

Dbf. Crufte cincree vefigia vix ulla. Scurella, ut guorundam crufaceorum Lichesum mos ef , absque crufa in lapide folutaria, rarus confertes aut congefta, difperfe inveniuntur.

99. LiCHen brunnens - cruftaceus gramulatus viri. difufcus fubglaucefcens; fcutellis confertis olivaceofulcis, margine pulverulento cruita concolore.

Patellaria brumea. Svet. Brumlaf. Lichen brumneus Swartz. Act. Upf, 4, Retz. Pr. 2. Liljebl.

Lichen multifloris Ehrh. Crypt, exf. Lichen badius Gmel. Syit. Limn. Lichen pezizoides Weber. Spicil. Dicks, Roth. Tent. Hoffm. Enum? Gmol, Sylt, Liun. Pfora brunnea. Hoffm. Deut. Fl.

Icon. Dicks. Cr. Britt. T. 2. f, 4.

Habitat fupra terram ad radices arborum \& ad latera montium fubitratis mufcis putrefactis. Obf: Variat crufter brunnea, fcutellis concoloribuis; - nec force specie difinguenda eft: 


\section{$-50 .-$}

Patellaria nebulofa Hoffm. Pi.Lich. T. 4o.f. r. qua:

Yora pezizoides Ejusd. Dout. Flo crufla Jubcinerea, foutellys depresfis rufofufcis.

100. Lichen erybe - cruftaceus fubpulverulentrs inolus virdis; fcutellis minutis lubimmerfis convexis totis rufofulcis.

Patellaria eryfbe svet. Eyglaf.

Habitat ad aseres femiputridos.

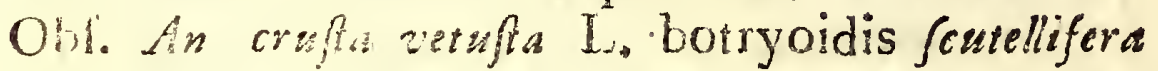
fat, dubutus hareo. Scutelle ob minutiem non facole dete conde nec fupra cruftam multum elevate.

101. Lichen effufus - crufaccus pulverulentus cinerens dithe aruginolus; fcutellis demum convexis xufefentibus, margine crulta concolore. Farellariat offula. Svet. Vidlaf.

I. chen Jalignus Schrad. Spicil, Fl. Germ.

Virucaria efufa hoffm. Deut, Fl.

Pubitat in Salicibus.

Obf Scutelle jumiores minute, plant, pallida, etians atate provecsitiores pallide fufca funt, fed denno rufelcentes or fufcornfe, conferta. Crufla lase effufa.

I02. LuCHEN viridefoens - cruftaceus granulofus viridtis; fcuielis demum convexis rugolis confertis difformibus fufco nig ris.

Palellaria viridefcens. Svet. Siubblaf:

Lichen viridefecns Schrad. Spicil. Gmel. Syft。 Linn.

- Verrucaria viridis Jioffm. Deut. El. Habitat in truncis arborum putridis.

103. LICHEN decoloratus - cruftaceus farinolo granulatus cinerafcers; fcutellis marginatis de. mum convexis fufcis \& nigricantibus. 
Patellaris decolorata. Sver. Knotterlat.

Lichen uranululus. Ehrh. Schrad. Spicil.

Verrucaria granubla. Hoffm. Pl. Lich, \&

Deut. Fl.

Patellaria decolorans, Hoffm. P1. Lich.

Verrucaria decolorans. Hoffu. Deut, Fl.

Icon. Hoffm. Pl. Lich. T. 30. f. 3.

Habitat lupra terram 8 in iruncis putrefcentibus.

Obf. Scutelle forma es colore in hac fpecie adtinodum variant, ut's etiarn cruple. Scutellas junio. res planas reprefentat Patellaria decolorans Hoffm. Pl. Lich. T. 30. f. 2. O quando cruffa leprola cinerea, fcucelias planis marginatss sninutis palludis Verrucaria incolorata in Ejousd. Deat. Fl. Ja. lutatur.

104. LICHen vernalis - cruftacens tenuistimus albovirefcens; fcutellis demm fubglobolis con. glomeratis fubincarnatis ferrugineisque.

Patellaria vernalis. Svet. Vârlaf.

Lichen vernalis, Lisnici.

Lichen Spharoides Dicks. Wither. Gmel, Sylt. Linn.

Verrucaria conglomerata. Hofm. Deut, Fl, Icon. Dicks.Cr. Britt.1.T.2. f. 2. (L. Cphroroides). Habitat lupra mufos \& cortices femipurridas. Obf. Occurrat Jcutellis athis, incarnatis, at fopis. fime rufefcentsbus er ferriagineo fufois, primo pla. nis margine concolore per atatem fere globolis. .

$V$ arietes cruflacea virefcens, foutslles minze. tis Jparfis concavis pallidis l. fufcis, demusm consvexis, mufcos ancruflians, vix pro diverfa fpecie habenda.

105. Lichen lencoftigma - cruftaceus fulco nigricans; glomerulis fpartis niveis; fcutellis convexis minutishimis rufofufcis, margine cinerafcente. 
Patellaria lescofigma. Svet. Huitprikslif.

Tabnat in cortice antigua \& rimola Quercus

Obf. Scutclia vix abique ecrilo armaro difingueis. de. Singularis fpecies of glomerulos puiverulen: tos albsismos sepe excavatos.

I06. LrCueN pyrinus - crultacens tenuis gramula. tus cinereo virefiens; foutellis minutis con. vexiufculis, margine cincrafcente.

Patellaria proma." Suet. Paroniaf.

Habiat in cortice ramomm iysi communis \& Mali.

* 7. Scutellis incarnatis \& fubrubellis.

107. Lichen rofillus. - crufaceus tenuis pulvera. lentus cinero abidus; foutellis tumidulis car. neis, margine albidiore.

Patellaria rofella. Svet. Blekroodlaf.

Lichen rofeilus. Perfoon. Uit. Ann. St. 7. Schrad. Spicil.

Lichen alboincarnatus Wulfo ap. Jacqu. Gnael. Sylt. Linn.

Verrucaria rolella toffm. Deut. FI.

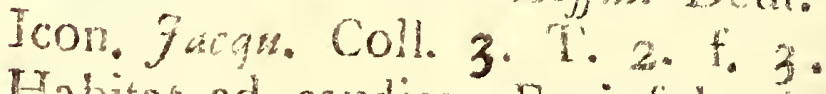

Habitat ad caudices Fagi fylvatica.

ro8. LICHEN anceps - cruftaceus farinolo-granula. latus viricis; foutellis dilute carneis, demun oblcure rufis, margine depresfo palidiore.

Parellaria anceps. Svet。 Omplkftslaf. Lichen anceps Ruifröm. Disfert.

Habitat in cortice arborum.

Obf. Strudura pracedentis o eidem affinis; tamen diffingui oportet. Margines foutellarum in jus nioribus convexi difcum luperanies concolores, in aeare magis provedtis depress 2 difco aguales $\dot{\sigma}^{\circ}$ vix nili colore panlulum dilutiori evidentes. 
109. LiCienen aruginofus - cruftacens granulofus glaucovirefcens; fcutellis carneis planis de. mum convexiufculis, margine undulato.

patellaria aruginofa. Svet. Rosilaf.

Lichen zruginofus Scopoli. Hay. Jacak. Fl. - Auftr. Hoffn, Enum, Liliebl. Gmel, Sylt. Linn. Lichen ericetorum var, fesilis Limn. var. B Lightf. var. carneus Retz. Prodr. 1.Schreb. Spicil, Withar. Arr. Weiso excl. Iynom.

Lichen elveloides Weberi. Alion. Roth: Tene. Gmel. Syft Linn.

Lichen icmadophila Ehrharti. Linn.Suppl, Retz. Prodr. 2. Liljebl. Hoffm. Enum. Gmel. Bylt. Linn.

Iichen viridatus. Gmel. Syft. Linu.

Lichen paraliticus Retz. Pr. I.

Icon. Facqu. Fl. Aultr. T. 275. Fl.Dan. T. 472. f. 4. Hoffm Enuin. Lich. T. 8. f, 1. Hag. Hilt, Lich, $T$, ז. f. 4. Dillen, Hif, Mufc, T. I8. f. II.

Habitat ad truncos putridos, in terra, locis turfolis paludofis, haud raro fupra Sphagnum. Obl. Pro ratione loci crufta jam glanca, jars magis virefcens; foutelle jars plance flexuofa, jam convexiufoula, lutefcenies.

IIO. LiCHen cupularis - crutaceus tenuis glaber cinereovirefcens; futellis concavis lutcorubellis, margine tugmido exalbido.

Patellaria cupularis. Svet. Skällaf.

Lichen cupularis Hedru. St. Crypt. Ehrh. Beytr. 4. Schrad. Spicil. Dicks. Bellardi. Schrank. Wither. Gmel. Syft. Limn.

Lichen fulcorubens Wulf, ap. Jacqu. Gmel, Sylt, Linn.

Lichen rubellus'Schrank. Bavar.

Verrucaria cupularis Hoffm, Deut. Fl,

Peziza 
Peziza jenentis Batfch. Gmel. Syft. Linn. Icon. Hedwig. Strip. Crypt, 2. T. 20, f. B. Hoffm. Enum, Lich. T. 6. f. 4. Jacgu. Coll. 3. T. 2. '. 2 .

Habitat in rupibus calcaris \& muris.

Obi. Variat crutta colore nigricante er rufefcente, l. nulla; foretellasgue exias hirus (an aifindta (pecies?)

11. LICHEN ulmi - cruftaceus pulverulentus albidus; fcuteliis contertis curcavis carneo rllbellis, margine albo pulverulento lubcrenia. lato.

Patellaria ulani. Svet. Almlaf.

Lichen ulmi Swartz, Nov, Aat. Upr. 4. Retz. P'r. 2.

Lichen marmoreus Dicks. Liljehl.Hoffm. Enum. Wither. Gmel. Sytt. Linn,

Lichen pallidus Hoffm. Enum, Lumaitz, Gmel. Sylt. Lim.

Lichen pruinofus Humbole. Frib. Lichen dufressin? Bellardi App. El. Pedem. Patellaria rubra Hoffm. Pl. Lich. Verrucaria rubra Hoffm. Deut. Fl. Icon. Hoffm. Pl. Lich. T. 17. f. 2. Ejusd. Enum. Lich. T. 5. f. 2. T. 6. f.

Habitat ad truncos arborum, Ulini, Fraxini, atque etiam aci latera rupium mufcofa.

Obf. Scrsellis femper conscavis, extus interdum hirfutis of difci colore dituze rubelio a Lo fubfulco perbene diftinetus.

* 8. Scutellis lividis E cinereoglausis plerrimque piuinofis.

12. LrCHEN ang zilo/aus - cruftaceus rimofus albicans; fcutellis marginatis fubcarneis glaucisque de. mum convexis glomerato congeitis difformibus, 
Patellaria angulofa. Svet. Grynlaf.

Lichen anigalofus Schrob. spicil. Rueftr. Disfo Hoffry. Hnum. Reach. Fl. Scholl. Gmel. Sylt. Litin.

Lichen pallidus schreb. Spicil. Hagen.

Lichen pallefcers Weis. IJ ulfen. ap. Jacqu.

Lichen. Lubcameus Swartz. Mefr. Liljebl. Retz.

Ir. 2. Achur. Ac. Sc. Svec, 1794.

Verrucaria pallida Hoffm. Deut. Fl.

Verrucaria angulofa Hoffm. Deut. Fl.

Icon. Zacqu. Coll. 3. T. 5. f. 3. a. (Achar. Nav. Act. Ac. Sc, Svec, T. XV. T.6. f. 4. is. Ubcamei.)

Tabitat in contice arborum \& faxis.

Obl. Sat polymorphus eft hic Lichen; pracipue ta - formam oj colorem receptaculorum Jpecles. Scutel. le juniores planoconvexa margine albicante of $h$. l. L. pallidus of pallefcens Auclorum. In lapide carne junt, margine faturatius indlo of criffe ambutus zonatus; In corticibus non raro

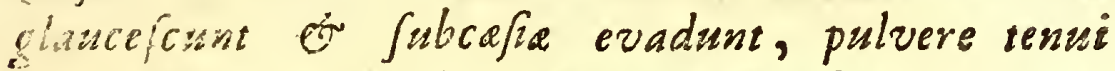
fere femper prumole; demum hemispharica angu. lofe, margine tvaruefcente.

An faxarilis (L. lubcarneus) Specie difin= "Etus, sis dubio eft.

II3. LICHeN Smartzi - cruftaceus albefcers anbitu lacinato, radiato-ramofo; fcutellis fub. globofis glaucis, atate convexiufculis marginatis agoregatis.

Patellaria Swartzii. Svet. Swartzlaf.

Lichen Swartzii Achar. Act. Sc. Svec. 1794. Lichen glancelcens $W^{\prime}$ efring. ACt. Sc. Svec, 1791. Liljebl.

Icon, hujus prodr. T, 1. f, 2. Nov, Ag. Sco Svec. T. XV. T. 6. f. 3 .

Habicat ad parietes rupium. 


\section{$-36 .-$}

Obl. Receptacula inde ab infantia funglohofa mizazra, (rarius plana es palluda) per aiatem pla. miora $\mathcal{O}^{\circ}$ tantum tumida funt, margine concolore conder, coacervata oj mutha presficise irregularia,

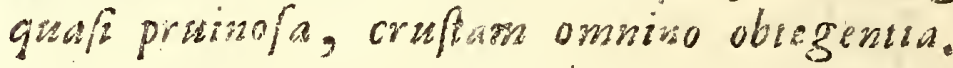

Y4. LICHeN impolitus - cruffaceus molus cinereo abidus; tcutellis adprestis planis marginatis cocoloribus, demum elevatis convexis pruia natis nigrisque.

Patllaria impolita. Svet. Mattlaf.

Lichen impolitus Ehrh. Crypt. Exf.

Lichen pruinatus Perfoom. Uit. Ann. St. II. Patellaria pruinata Perfoon. Ut. Ann. St. 7 .

Verrucaria impolita Hoffm. Deut. Fl.

Habitat in rupibus \& faxis.

Obf. Differt a I.. Swartzii crufte margine non

- fimbriato foutellis jurioribus planis fubimmerfis, per atatem hemispharicis, concoloribus \& demum omnino nigris.

IIs. Lichen glaucoma - cruftaceus rimofus cinerefcens; fcutellis demun convexis difrormibus congeftis fubcæliis pruinolis, margine flexuofo crenulato crufta concolore.

Pateliaria glancoma. Svet. Klipplaf.

Lichen fordidus Perfoon. Uft. Ann. d. Bot. St. \%. Lichen albidocrefius, Schrad. Spicil, Fl. Germ. Verucaria glaucoma Foffm, Deut. Fl, \& Pl.Lich. Icon. Hoffm. Pl. Lich. T. 52. 53 .

Mabitat in faxis \& rupibus,

Dbf. Hic forte idem ef ac:

Jichen rupicola Linnai, quem itidem nominarsing Retz. Liljebl. Hudf, Lightf. Oed. Emasm. Leers Zoega, Roth. Gmel. 
* 9. Scutellis carulefientibus ES nigrocafis plerumque pruinofis.

II6. Licuen Dilleniamus - cruftaceus mollis gyrofo verrucofus granulatus rofeus; fcutellis pruinofis nigro calits, margine nigro.

Patcllaria Dilleniara. Sver. Dillenslaf.

Icon. Tab. noftra I. f. I.

Habitat ad latera montium.

Obf. Inter crufaceos fingularis fpecies ob mollem iexturam. Odor foavis Viola 1 . radicis Ireos florent. diu perdurans.

117. LICHEN abietinus - cruftaceus tenuisfímus lævis glaucefcens; fcutellis planis marginatis pruinofis nigrocæiffis.

Patellaria abiesina. Svet. Granbarkslaf.

Lichen abietinus Achar. Adt. Sc. Svec. 1795. Icon. Achar. Nov. Act. Ac. Scient, SV. T. XVI, T. I $5 \cdot$ f. 7 .

Habitat in fquamis corticis Pini abietis.

I 18. Lichen Hageni - cruftaceus tenuis cineralcens; fcutellis minutis pruinofis nigrocæilis, margine albo.

Patellaria Hageni. Suet. Hagenslaf.

Lichen cierulefcens Hagen. Hif, Lich, Hoff fro.

Enum, Roth. Tent. Gmel. Syft. Linn.

Verrucaria cærulefcens Hoffm. Deut, Fl.

Icon. Hagen. 1. c. T. I. f. S.

Habitat ad cortices arborum juniores.

Obf. Ab hoc vix differt:

Lichen fambuci Perfoon. U/t. Ann。 d. Bct. Sto 7. criffar viridi cinerea; fcruellis carshleis.

I19. Lichen corticola - cruftaceus rugulolo rimolus candidus pulveralentus; fcutellis demum fub. globolis confertis minutis marginatis nigris glauco pruinolis.

D 5

$P a$ - 


\section{$-58 .-$}

Fatellaris corticola. Svet. Pricklaf.

Lichen corticola Achar. Act. Sc. Svee. 1794.

Lichen anylactis Ehrh. Cr, exf. Perfoon. Ut. Ann. St. II.

Iichen alboater Hoffm. Erum. Schrad. Spicil. Gnel. Syfi. Linn.

Verucaria inylace fioffm. Teut. Fl.

Verrucania a L raur Hiffm. 1]. Lich. \& Deut. Fl. Splizria tysiacra Weigel. Cbl.

Icon. Hloffm. P. Lich. 1. 5. t. 2. Achar. Nov. Ad. Ac. Sc. Siec. T. XVI. T. 9. 1.6.a.b.c. Habitat in cor ice vetuna siu ola (uercus, Fraxini.

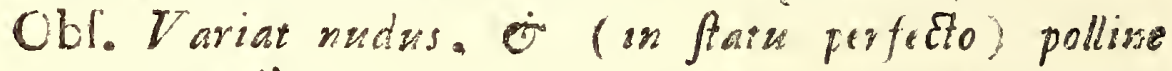
glanco albo totus fuffurus, unce foutclla cofjec apo parent.

Cum L. melaleuco of $I$, notho non confundendus.

120. IICHFN epipoims - cruftaccus glebulofo fubin bricatus candidus; fourellis narginatis denum convexis callis pruirolis.

Patellaria epipelia. Svet. Rimlaf.

F abitat ad larides calcarics, in muris.

Obf. Cum hoc noftro bexe convenit:

Iichen intermeditis Schrad. Spical Fl. Germo

A L. candido Web. defencta lpecies certe eft.

121. Lichen fulphurens - crupacevs rimofus toruJofus fuiphouetus; Icuellis convex is nigricantibus pruncfs nargine palliricoribus.

Patcllaria Suiphurea. Svet. Svafuellaf.

Lichen fulthure us Foffm. I rum. Lich. Dicks.

Schrad. Spicil. Wher. Cmel. Sylt. Linn.

Lichen calcirius Leers. Hexborn.

Verrucaria fulphure: Hoffm.Pl. Lich, \& Teut.Fl. Icon. Hoffm. H]. Lich, 1. 9. f. 3. Ejusd. Entum,

Lich. T. 4 f. 1. Habitat in rupibus. 
122. Lichen amylacess - cruftaceus fubrinofus albomargaricaceus; fcutellis fuhmmeris planis demun erumpentibus convexis nigris albo. pruinolis a crufta maruinatis.

Patellaria amylacea. Svet. Starkzlfelaf.

Lichen anylaceus Achar. Act. Sc.Svec.'I79\%. Retz. Pr. 2.

Icon. Achar. Nov, Aet, Ac. Suient. Svec. T. XV. T. 6. F. 6 .

Habitat in faxis 8 montibus elatis.

ObC. Colore es craita latius effuta a L. Pulphireo differt hic nofler, guamvis habitus fere idem. Afinis etiam L. fpeires of calcario fed Specie diverfus videtur.

123. Lichen albocarulefcens - cruftaceus albus æqua. bilis contiguus; fcutellis elevatis planis caru. lefcentibus, margine nigro.

Patellaria albocarslefcens. Svet. Huitblalaf.

Lichen albocærulefcens Wulfen ap. Jacqu. Gmel. Sylt. Linn.

Patellaria albocar lefcens Hoffm. Pl, Lich. Verrucariz albocarulefcens Hoffm. Deut, Fl。

Verrucaria annulata Hanbole. Erib.

Icon. Facqu. Coll. 2. T. I5, F. I. Hoffm, Pl。 Lich. T. 14. f. 2.

Habitat ad rupes.

Obl. Cruta alba non rimola; (cutelle margine fube tus a crufta folute of elevate, confeanter plana..]

124. IICHen fpeireus - cruft ceus fubcontiguns albisfinus; farellis planis céfo pruinois de. mum convexis totis atris

Patellaria Sperrea. Svet. Gipslaf.

Habiat ad rup's marinas.

Obf. Medius quafi inter L. albocrerulefcentem eto L. calcarium, Differt ab illo: Jcutellis fescalabus 
séc elevatis in infantio depressis, immarginatis of per etaten magis convexis, atris, plerumque marsine albo a crupla proveniente, quaf cinclis; ab hoc vero: fcucllis denum majoribus, primum cecies of pruinofs.

* 10. Scutellis atris.

25. LiCHEN calcarius - cruftaceus rimolo areola. tus fubpulverulentus albislimus; fcutellis de. presis minutis f parfis demum convexis totisatris. Palellaria calcaria. Svet. Kalklaf.

Lichen calcareus Linnci!

Habitat in laxis præcipue calariis \& muris antiquis. Obf. Crufta per atatem minus alba of fere fordida obvenit.

Hic of L. confluens promifcue turus pro al. tero apud Auctores occurrit, unde fynonyma valde ambigua. Genuinam tamen hanc esfe Linnai Speciem varia tefantur in herbariis quorundams exemplaria, manu Ejus propria fignata. Vix Specie differunt:

Verrucaria calcaria Hoffro. Deut. Fl. crufta effufa lactea, foutellis convexis atris a crufta marginatis; alque

Lichen cretaceus Ehrh, Beytr. crufta farinoja cretacea.

126. LICHEN confizens - cruftaceus rimofus glauco cinerafcens; fcutellis marginatis demum convexis confluentibus totis atris.

Patellaria confluens. Svet. Groddlaf.

Lichen confluens Weberi. Swartz. Retz. Pr. 2. Liljebl. Dicks. Hoffm, Enum, Roth. Tent. Willars. Wither. Bellardi. Gmel. Sylt. Linn. Verrucaria confluens Wigg. Hoffm, H, Lich, \& Deut. Fl.

Verrucaria nigro cinerea Markim. 
Icon. Weber Spicil. T. 2. Hoffm, Pl, Lich, T. I9. f. I. Mich, Gen. T. 54. f. 7. Habitat in rupibus \& faxis.

Obl. Variat crufta magis alba es obfcure cinerea, nec non fuperficie ochracea, atgue crufia plase nulla.

127. Llichen lapicida - cruftaceus rimolus areolato granulatus cinereo glaucus; fcutellis depresfis planis marginatis totis nigris.

Patellaria lapicida. Svet. Stenfraislaf.

Lichen petrrus $W$ ulf. ap. Jacqu,Gmel. Syft,Linn. Lichen concentricus Davies Act. Soc, Linn. 2. Sowerby. Linck. Annal. Nat.?

Verrucaria petræa Hoffm. Pl. Lich, \& Deut. FJ. Verrucaria contigua Hoffm. Deut. Fl. Verrucaria cicatricola Hoffm. Deut. Fl. Verrucaria pantherina Hoffm. Deut. Fl. Verrucaria tigrina Hoffm. Deut. Fl. Icon. Facqu. Coll. 3. 'T. 6. f. 2. Hoffm. $\mathrm{Pl}$. Lich. T. 50. Sowerby Engl. Bot. T. 246. Habitat in faxis arenariis calcariisque.

Obf, Variat crufece colore candido, lacteo, cinerafcente of fubcafio, fcutellisgue concentricis, ex. centricis, contiguis of a crufta margikatis, Sape medio impresfis. Cruftá junior fuborbicularis, demum difformis of graxdava perguam cras $\int_{a}$ evadit, of maximam partem intus rubra.

128. LJCHeN ocellatus - cruftaceus rimofus areola. tus cinereus; fcutellis depresfis nigris, margine elevato crufta concolore fubocellatis.

Patellaria ocellata. Svet. Ogonlaf.

Lichen ocellatus Retz, Prodr. Fl.' Sc. 2.

Verrucaria ocellata Hoffm.Pl, Lich, \& Deut. Fl.

Verrucaria ocellulata, Hoffm. Deut. Fl, Icon. Hoffm. Pl. Lich, T. 2O, f, 2. Habitat ad lapides. 
129. LICHEN rimofus - cruftaceus albicans xquabilis rimofisimus, areolis angulatis; fcutellis atris glaucis marginatis.

Patellaria rimofa. Svet. Tarningiaf.

Lichen rimofus Retz. Pr. 2. Dicks. Gmel. Syit. Linn.

Icon. Fl. Dan. T. 4 KR. f. 2.

Habitat in faxis ad latera fluriorum alpin. Obf. A L. tesfílato diftenguendus.

130. Lichen cyathoides - cruftaceus rimofn fub. verrucolus cinerafcens; fcutellis elevatis planoconcavis flexuolis pruinotis toris nigris.

Patellaria cyathoides. Svet. Skidillalaf.

Habitat in faxis \& rupibus.

Obl. Scutelle parva tenues \&o quaf membranacere,

- crufta parum eievata os joluta, bacillo brevistimo ad centrum affive criufte, rapius pruinofa, per alatem margine undulation flexa.

Occurrit in lapimentis ligneis antiquis alius, grem foecre vix diffinitum puto, foutellis bacilo.

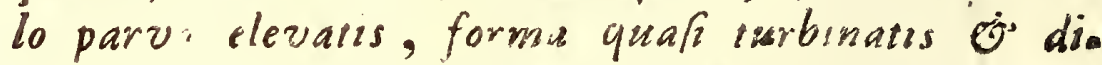
foo planis vel aliquantum convexunfculis.

Hujus loci forfan erie:

Verrucaria capitellata Bofjm. Deni. Fl。 a L. cinerco cerie difinguinda. cfr. Hoffm. $l . c$.

13I. Lrches atralys - cruftaceus; fcutellis planiufculis aggregats maryinatis totis. atris. Parellaria airata. Svet. Kollaf.

Lichen atratus Hedwig. Stirp,Cr,Gmel.Sylt.Linn. Icon Hedruig I. c. 2. T. 21, f. a. Habitut in arborum ramis emortuis \& putre. fcenibus, cuticula rupta \& elevata.

33. Lichen mifcellus - crutzacelis rimofus rugofo gramulatus cinereo cefilus nigro variegatus; fcutellis marginatis demum convexis totis atris. 


\section{$-63 .-$}

Patellaria mifcella. Svet. Blandlaf.

Habitat in faxis \& rupibus.

133. Licher atroalbus - cruftaceus niger albo verrucofus; fcutellis marginatis demum conve$x$ is immixtis totis atris.

Patcllaria atroalta. Svet. Svarthvitlaf.

Lichen atroalbus Linn. Thunb. Jap. Retzo

Pr. 2. Loljebl. Hoffb. Huds. Web. Hoffms.

Enum. Roth. Tent. Bellards. Walf. Wuther.

Lightf. Gmel. Syft. Linn.

Verrucaria atro alba o cinereoatra Hoffmo

Deut. Fl.

Icon. Wulf. ap. Jacqu, Cóll, 2. T, 14, f, 4. Habitat in faxis.

Obl.Scutella rarius \& vix nifzoculo armato ab fervanda.

134. IICHen fufcoater - crulfaceus aterrimus, areo. lis nitidis adprestis nigrofufcis; fcutellis marginatis de mum convexis immixt is tot is atris, Patellaria fuccoatra. Svet. Swartbrunlaf.

Lichen fufcoater Linnai.

Habitat in faxis.

Obr. Scutelle on interfitiies areolarum atre, opace, minute, rarius occurrunt es vix nifi microfcopio detegende, ab arcoles nitidis fufcis diftindisfima.

Synonyma fere omnia Azetorum, qui áe L. fufcoatro meminerunt, panca fi fortasfit exceper rim, vaga es incerta, vel ad L. umbrinum vel L. diffractum referenda; vixque differt:

Lichen carbonarius $W$ ulf, ap, facqu. Coll. $3_{0}$

T. o. f. 2. b. crufta farinucea, uberculisque fpharoideis $\mathcal{F}^{\circ}$ intermixtis foutellis atris. Gmel. Syft. Linn. cfro etiam Wulf. in Bea Jchaff. d. Berl. Gef. Nat. Fr. 8. p. 53.

135. Lxcurn diffractus - crupaceus rimofus olivam ceofufcus, areolis nigromarginatis; fcutellis 
tellis demun convexis coufluentibus totis atris.

Patellaria diffracta. Svet. Bräcklaf.

Habitat ad lapides \& muros antiquos.

Obl. Hunc juniorem plurimi forfan-Aullorum pro

L. fufcoatro habuerune.

136. Lichen citrinellus - cruftacens giranulato pulverulentus flavovirefcens; fcutellis minutis fubglobofis atris.

Patellaria citrinella. Svet. Citronlaf.

Lichen citrinellus Achar. Act. Sc. Svec. 1795. Icon, Achar. Nov, Act. Ac. Sc, Svec, T. XVI. T. 5. f. 5 .

Habitat fupra terram fubftratis mulcis putrefaotis ad juga mont. alp.

Obf. Varieras hujus eft:

Lichen flavovirefcens-Dicks. Crypt. Britt. 3. T. S. f. 2. crufla magis vire/cente of foutellis vetuftioribus majoribus rugofisque.

I37. Lichen parafemus - cruftaceus tenuis fubgra. nulofus albicans cinereoque virefcens nigro fublimitatus; fcutellis planiufculis marginatis totis atris.

Patellaria parasema. Svet. Grintslaf.

Lichen fanguinarii varietas Linnai of Audtorum.

Lichen punctatus Scopoli Fl. Carn.

Lichen limitatus Scop. Carn. Willars, Bellardi.

Lichen rugofus var. $\beta$ Weis. Pl. Crypt.

Verrucaria punctata Hoffm. Deut. Fl.

Sphreria lichenoides var, $\beta$ analtomofans Weig. Obf.

Icon. Hoffm. Enum. Lich.'T 5. f. 3. 5. Jacqus. Coll. 3. T. 5. f. 3. b. Weig. Obf, T.2. f, 13. Dilles. Hift, Mufc, T. I8. f. 3.

Habitat ad arborum corticem \& faxa.

$\mathrm{Obl}$ 


\section{$-65 .-$}

Obf. Differt ommino a L. fanguinario Fautellis plerismque planis intus concoloribus es crufla for ma ac indole. Neque in L. fanguinarium abire certum eff.

Paristates hujus precipue funt:

Verrucaria limitata Hoffm. Dent. Fl. cruffali. nea repanda fepta eso decussata. Undigue in cortice, in laxis rarins.

Verrucaria guttata, Hoffm。 Devt. Fl, cruffacio nerea, fortacllis guttatis. In faxis; etians e. eruffacens.

Verrucaria olivacea Hoffm. Dent. Flo cru. fia e cinereo dilüle olivaceo vircfcente. In faxis fordide olivacea. Scutella hinc inde fuffurfercentes.

138. LICHEN Janguinarins - cruftaceus rugofo verrucollus glauco cinerafcens; fcutellis hemifphrricis atris intus fanguineis.

Patellaria Janguinaria. Svet. Blodlaf.

Lichen fanguinarius Linn. Thunb. Japon. Retz.

Pr. 2. Liljebl. Huds. Lightf. Scholl. Leers. Schreb. Spicil. Pollich. Hiag. Neck. Reich. Fl. Mattufco Mohr. Schrad. Spicil. Ehrh. Hoffm. Enum. Bellardi. Roth. Tent. Leyss。 Wither. Gmol. Sylt. Linn.

Patellaria fanguinea Perfoon. Uit. Ann, d. Boto St. 7 .

Verrucaria fanguinaria Hoffm. P1. Lich. \& Deut. Fl.

Icon. Hoffm. Pl, Lich. T, 4r. f. I. Sowverby Eng. Bot. 3, T. 153 .

Habitat in rupibus \& cortice arborum.

Obf. Differentia hujus a L. parafemo ex chara. ctere amborum Specifico lacis patet, licet hand rite olim permixis fuserins.

$\mathbf{E}$ 


\section{$-66 .-1$}

139. LICHEN pimicola - cruftaceus tenuis inzqualis effufus albocinerafcens; fcutellis planiufculis minutis totis atris.

Patellaria pinicole. Svet. Tallaf.

Habiat in Pino fylvefri \& Alno.

Ob. A I. parafeno habitu atgue foutllarum in. dole difincius; fed forte non differt:

Verrucaria punctiformis Hoffm. Deut. Fl.

140. Lichen Oederi - cruttaceus tenuis rimolo a. reolatus rubro ferrugineus; fcutellis minutis fubglobolis fubimmerfis plerumque pertufis nigris.

Patellaria Oederi. Svet. O̊derslaf.

Lichen aderi-Linnai. Swartz. Act. Upf. 4.

Lichen Koenigii Gunn. Retz, Prodr.

Icon, Fl. Dan. T. 470 . f. I.

Habitat in alpibus feptentrionalibus.

Obf. Ob foutellas pertusas Verrucariis propinguns. Andteribus minus nota neque ab iis rite determi. nata pecies.

An color crufta hujus \& Sequentis Jpeciei pro. prius, l. ex minera ferri provenicns?"

I4I. LICHEN filaceus - crufaceus verrucofus lævis ruberrimeis fcutellis marginatis demum convexis totis atris.

Patellaria filacea, Svet. Ochralaf..

Lichen oederi Liljebl. Web. Dicks Schrad. Rel-

lardi. Huds. Wither. Hoffm. Enum. Roth. Tent. Lichen Konigii Retz. Prodr. 2. Humb. Frib. Pacellaria flacea $B$ offm. Pl. Lich. Verrucaria hlacea Hoffm. Deut, Fl. Verrucaria celeri Hoffm. Deut. Fl. Icon. Hoffm. Pl. Lich. T. I9. f. 2.

Habitat fupra faxa, graniten \& durisfimas petras. Obl. Ne confundatur cum L. confluente colore ochraceo inierdum tincto.

I 42. 
142. Lichen hadius - cruftaceus verrucofo rimofus glaber olivaceo fufcus glaucefcens; fcutellis nigris, margine crufta concolone.

Patellaria badia. Svet. Gldinslaf.

Lichen badius Perfoon. Uft. Ann. d. Bot. St.7. Lichen fulcatus Schrader. Spicil. Fl. Germ.

Verrucaria badia Hoffm. Pl. Lich. \& Deut. Fl. Verrucaria grifeoatra Boffm. Deut. Fl.

Icon. Hoffm. PI. Lich. T. ST. f. 2 .

Habitat ad faxa calcaria, bafalina.

Obf. Crufla initio plana, fubfoibacea, fcutellis im. merfis, per atatcm coacervata, irregularis, fcu. tellis protuberantibus, interdum cinerafcens l. glatuco virefcens.

143. Lichen lophodes - cruftaceus granulatus cinereo virefcens orbicularis; fcutellis nigris, mar. gine crufta concolore.

Patellaria Jophodes. Svet. Skymmellaf.

Habitat in Tilix \& Betulæ cortice laviori.

Obf. Crufta. ex meris verrucsalis compofica, parva, orbicularis, fu/co limitata, grandava irregularis. In fingula verrucula foutella progerminar, unde receptacula in medio crupe confertusfara, fenform. gue verfus ambitum propullulantia. Loco cre. fcendi of cruspa forma a priori difizinctus.

144. Lichen tigillaris - cruftaceus rimofus lavis thavicans, verrucis crutta elevatis fcutallife. ris; fcutellis plunis totis nigris.

Patellaria tigillaris. Svet. Sparriff.

Lichen viridefcens Liljeblad Sv. Fl.

Habitat ad trabes \& fapimenta lignea fubpiz trefcentia cum L. vario aliquando asfociatus.

145. Liches tephromelas - cruftaceus rugofo gro nulatus albicans; fcutellis tumidolis atris. margine albo demum flexuolo crenulato. 
Patellaria suphromelas. Svet. Kruskantlaf. Lichen tephromelas Ehrh. Crypt. t:xf.

Lichen ater Hudfoni. Retz. Fr. 2. Schreb, Spio cil. Hoffim. Enum. Lighef. Reich. Fl. Wild, Prodr. Roth. Tent. Wisher. Lumnitz. Gmel. Syit. Linn.

Lichen niéricans Necker. Gallob. Hagen.

Lichen cinereus Wulf. ap. Jacqu.

Lichen cineralcens Gmel. Sylt, Linn.

Iichen melanotichtos Gmol. Syft. Linn.

Lichen fubfufcus Hoffl. Vaxt. R; var, ater

Retz Prodr. I; var, a. ater Weis. Crypt var. $\beta$ ater Pollich.

Verrucaria atra Hoffm. Dout. Fl.

Verrucaria atrata. Hoffm. Deut. Fl.

Icon facqu. Coll. 2, T. 14, f, 5. b. Dillen. Hift. Wufc. T. 18. F. 15.

Habitat in rupibus \& cortice arborum.

Obl. A L. fubfulco vix differt, nili crufla mas gis leprofa of verrucofa, fcuellisquie a prima in. fantia atris, lepe coacervatis 'fo per ataiem am. pliortbus, flexnojss, margine laxo undulato cre. neslato.

146. LICHEN grmmolus - cruftaceus fubfarinofus cærulefcenti cinereus; fcutellis fubininerfis con: vexis nigris, margine rugorn alboficente. Patellaria grumoja. Svet. Gribiálaf. Lichen grunofus Perfoon. Utit. Annal, d. Bot. St. 11. p. IS.

Lichen carulefcens Perfono ibd. St. 14. p. 34. Verrucaria grunora Hoffm. Deut. Fl. Habitat in laxis

Obfi. Collatus meis cum $\mathrm{Cl}$. perfoonii obfervationi. bus, multa fordeni, ut hic speciem propriams conflumere debeat. 
147. IICHEN exiguns - cruftaceus oblcure cinere. ns; fcutellis minutis aggregatis atris planis, margine albo crenulato, demum convexis margine evanido.

Patellaria exigua. Svet. Smalaf.

Lichen, conticolse varietas Achar. Aet. Sc.

svec. 1795.

Icon, Achar, Nov, Ag, Ac. Sc, Svec, T.XVI. T. s. f. 6. d. e. f.

Habitat in cortice rimora Quercus \& ad parie. tes ligneos veturios.

Obl. Grandavus factus varieranbus quibusdam L. parafeni $e^{\circ} \mathrm{L}$. pinicolas pergam fimilis, in his vero fourelle femper planiz/cula marginate concolores. Vix differt a L. abietino Ehrh. nila magninitudine vo infra $L$. pericleum.

I48. Lichen uliginofus - crufiaceus grannulofus fer. rugineo fricus; fcutellis marginatis demunz convexis conlertis totis nigricantibus.

Patellaria uliginola. Svet. Sismplaf.

Lichen uliginofus, Schrader spicil, Fl. 'Germo Verrucaria uliginola Hoffer. Deut. Fl.

Verrucaria humofimilis Hoffm. Deut. Fl.

Habitat fupra terram \& mulcos putrefactos 10. cis murofis uliginofís.

Obf. Variat crufa cinerafcente fcutellisque conflus entibus, nec ab hac fpecie facile diffingunutur: I.ichen humolus Ehrh. Crypt. exr. $\dot{\sigma}$

Verrucaria humola Hoffom, Dent. Fl。 nifa crufas nigricante.

I49. Lichen mufcorum - crultaceus pulverulentus albicans; fcutellis marginatis demum hemisphe icis contiguis totis atris.

Patellaria mu/corum. Svet. Mosslaf. Lichen mulcorum Weber. Linn. Szyartz, Retz. $\mathrm{Pr}$ 
Pr. 2. Liljebl.Wulf. ap. Jacqu. Relh. Roth. Tent. Bellardi. Willars. Lumnitz. Wither. Gmei. Syit. Linn.

Lichen mucoriformis? Scop. Carn.

Verrucaria mufcorum Hoffm. Deut. Fl.

Icon. Relhan. Cant, p. 424. tab. Facqu. Coll. 4.

T. 7. f. I.Fl. Dan. T. 100\%. f. I.

Habitat ad terram fupra mulcos.

Obf. Crufia L. incani, an hujus?

1.50. Lichen immerfus - cruftaceus lævis albidus;

fcutellis immerfis atris deciduis.

Patellaria immer/a. Svet. Hâllaf.

Lichen immerfus Weberi Spicil. Schrad. Spicil.

Relhan Cant, Schrank. Wiiher. Roth. Tent.

Bellardi. Horfm. En.

Lichen calcivorus. Ehrhart Crypt. exl.

Verrucaria immerfa Hoffm. Pl. Lich.

Verrucaria calcivora Hoffm. Deut. Fl.

Icon. Schrad. Spicil. Fl. Germ. T. I. f. 7. a.

b. Sowerby Engl. Bot. 3. T. 193. Hoffm, Enum. Lich. T. 3. f. S. Ejnsd. P1. Lich. T. I2. f. $2-4$.

Habitat ad lapides calcarios nec non in lapi.

de magis duro.

Obl. L. calcivorus differt Jcutellis planinfoulis.

\section{* I.}

$\because$ L. umbilicatus - cruftaceus cinereus; fcutellis minutis candidis umbilicatis. - Verrucaria um. bilicata Hoffm. Deut. Fl. cujus varietas:

Lichen albellus Perfoon. Uit. Ann. St. nI \& Verrucaria albella Hoffm. Deut. Fl. crufta lactea fcutellis concavis concoloribus vel ad lentem dilco lubcarneo, Cort.Quercusjun. Patellaria umbilicatai rup. faxa. t $\mathrm{L}_{0}$ 
+ Lo circumforiptus - cruftaceus canus, margine fer. pentino atro; fcutcllis difci lentiformibus concoloribus, - Lichen circumicriptus Wulf. berl. Nat. Schrift. 8. Gmel. Sylt. Linn.

Patellaria circumjcripta.

+ $L_{0}$ urcelaris _ cruftaceus cinereus viridisque; fcutellis concavis pallidis, - Lichen urceolaris Gmel. Syit. Linn. Schrank, Fl. Bav. 2. p. SI4. Patellaria urceolaris.

+ L. verniciformis - cruftaceus verniciformis glauco virefcens linea dupplici circumicriptus; fcutellis planis pallidis - Lichen verniciformis Roth. Tent. Gmel. Sylt. Linn.

Obf. Forte varietas L. fubfufci \& idem cum L. byslino Dicks.

Patellaria verniciformis. cort, jun. Fagi.

f L. nigrovirens - cruffaceus gelatinofus niger; fcutellis minutis planis atprestis pallide viridibus, margine albo. - Lichen nigro.virens Schrank. Prim. Salisb.

Obf, Pro L. antiquitatis fructificante habet. Patellaria nigrovirens. lap. \& lign, antiqu.

$* 2$ 。

+ I, ochrochloras - cruftaceus albicans; fcutellis Ixvibus fubglobnis olivaceo flavis. - Lichen ochrochlorus Gmel. Syft. Linn. - Lichen viridiflavefcens $V$ ulf, ap. Jacqu. Coll. 3. T. 2. f. I. Obf. Affinis Lo irrubatio v. infra.

Patellaria ochrochlora. "faxa.

t L. unicolor - cruftaceus verrucofus flavus; fcutel. lis concoloribus marginatis immarginatisve, Lichen flavus Bellardi-App. Fl. Pedem, Obr. An. diftinctus a L. lucido? Pacellariza unicolor. ' faxa, 
* I. poiyeropus - cruftaceus lavis rimofus flavovi. refcens; futellis plano convexis carneo flave. centibus - Lichen polytropus Ehrh. Cr. ExT. - Lichen atrovirens Wuif, ap. Jacqu. Coll. 2. T. 14. f. 2. - Verrucaria polytropa. Hoffmo Deut. Fl.

patelinis polyeropa. faxa.

\section{* 3 .}

$\therefore$ L. calous - crutacens albidus nigro porulofis; fcutelis pulvinatis fpar is abris nitidis oblcure fulvis. - Iichen calvus Dickf. Cr. Britt. 2. T. 6. f. 4. Gmel. Sylt. Linn.

paellaria calva. rup.

t L. fulum - crufacens hateo viridis ; foutollis fulo vis. - Lichen fulvus Schreb. Spicil. Hoffm. E. num. Scholl. Lumnutz. Gmel. Sylt. Linn, - Ob. fcura fpecies.

Patellaria fulvs. ad falices.

t L. fufcoluten - crufaceus oranulofus glebuhofus albidus; fcutellis planis fordide luteis pruinofis obfoInte marginatis. - Lichen fufcolutens Ducks. Cr. Br. 2. T. 6. f. 2: Gmel. Sylt. Linn. Dbf. Farina deterfa futella nigricantes, mar. ginibus per lentem confpicuis.

patellaria fufcolntea. ad Alp. fupra mule, $\&$ platit. emort.

$t_{i}$ L. jungermannie - cruftaceus cinereus; fcutellis convexis fulvis margine plano conco. lore. - Lichen jungermanniz Fl, Dan. T. 1063. f. I. Retz. Pr, 2.

Patellaria jungermannia. in Jungerm. ciliari.

t I. ochroides - cruftaceus farinofus cinereo albidus; Icutel. 
fcutellis crasfufculis concavis flexuolis margi. natis totis lutefcentibus - Lichen lutelcens Perfoon. Uft. Ann. d. Bot. St. 7. Patellaria ochroides. Laxa dura.

t L. guadricolor - crufaceus pulverulentus tenuis grifeo albidus; fcutellis planiufculis lutefcentibus demum nigris. - Lichen quadricolor Dicks. Crypt. Br. 3. T. 9. 1.3.

Obf. Scutellæ juniores lutefcentes albo marginatz. Patellaria quadricolor. terra mont.

* 4 .

t L. alboflavejcens - cruftacens nodulofo verrucofus; fcutellis aurantiis, margine tumidiufculo albo. - Lichen aboflavefcens $W$ ulf. ap. Jacqu. Coll. 3. T. 5. f. 1. Gmel. Sylt. Linn. Patellaria alboflavefcens.

† L. flavovirefcens - cruitaceus flavovirefcens; fcu. tellis fafco aurantiacis margine flavo. - Patella. ria flavovirefcens Hoffm. Pl. Lich, T.2O. F. I. - Verrucaria flavovirefcens Hoffn. Deut Fl, - Lichen flavovirefcens? Wulf. ap. Jacqu. Coll. 2. T. 13. f. 4. b. Gmel. Sylt, Linn. Obf. Forte id. cum L., falicino? Patellaria flavovirefcens. rup.

4. L. holocarpus - cruftaceus aurantiacus; fcutellis confertis rufefcenti aurantiis. - Lichen holo. carpus Ehrh. Cr. exf, - Verrucaria holocarpa Hoffm. Deut. Fl.

Patellaria holocarpa, fapiment. lign.

t Lo citrinus - crultaceus pulverulentus citrinus; fcutellis aurantiis minutis margine flavo. Verrucaria citrina Hoffm. Deut. Fl. cujus varietates: E 5

Ver- 
Verrucaria fulva Hoffm. 1. co crufta citrina, fcutellis fulvis; \&

Verucaria flava Hoffm., 1 c. crufta flava, fcutellis fparfis minutis lividis,

Obi. An id. cum L, esythrello?

Petellaria citrina. lax. rup.

t L. obliteratus - cruftaceus glebulofus flavefcens; fcutellis contertis convexiulculis rufelcenti aurantis - Lichen obliteratus perfoon Uit. Ann. St. 11. - Verucalia obliterata? Hoffm. Deut. Fl. Obl. Crufta in medio a fcutellis angulatis confertis obliterata, in margine quaí foliacea.

Verrecaria viridiufa Boffm. Deut Fl. flavovio refcens, fcutellis vargmatis rufelcenti auran. - tiis; ut fubpecies L. obliterati ab Eo defcribitur, \& ut fynonymon aufeıtur L. flavovireicens Wulf v. fupra ad L. flavovirefc.p. 73. Patellaria oblstrata. rip. faxa.

+ L. rutilus - crufacens nigricans; fcutellis globofis faturate aurantiis - Lichen rutilus $W$ ulf ap. Jaccu. Coll. 3. T, 6, f, 2. c. Gmel, Sylt, Linn. Pateliciria rutila.

\section{* 5 .}

$\div$ L. gelias - crufaceus fubrugofus fuborbicularis pli. catus rimofus albicans; glomer tis centralibus; fcutellis concavis rubı is a crulta marginatis - Lichen gelidus Linn. Mant. Retz. Fr. 2. Gunn. Dicks. Willars. Bellardi. Waki. Act. Soc, h, n. hafn. 3. 2. 161. Gmel. Sytt. Linn. - Lichen Hecla Gunn. Fl. Dan. T. 470 . f. 2. Patellaria gelida. rup. alp.

f L, Vahiii - cruftaceus pulverulentus lacteus;; fcutellis elevatis coccineis, margine, integro. - - 


\section{二 $75 \cdot-$}

Lichen Vahlii Retz. Prodr. 2. - L. lacteus $V$ ahl. N. Hilt, S, Skrift, 2.

Patcllaria Vahlii. mont.

+ Lo irrubatus - cruftaceus lividas; fcutellis ninutis rufis marginatis - Lichen rufefcens $\Gamma$. Dan. T. 825 . F, 2.

Obf a L. rupeftri difinctus videtur. V. s. 1.

Pareliaria irrubata, faxa.

* 6 .

+ Lo coronatus - cruftaceus cinereus granulolus; fcutellis rufefcentibus depresfis a crufta granalofo margine cinctis, difco convexo. - Verrucaria coronata. Hoffm. Delut. Fl.

Obl. Nimis afinis L. microphyllo. An difingtus? Patellaria coronata. rup. terra.

4 L. cyane/cens - cruftaceus lievis albidus; fortellis fubflexuofs pruinais planis furcis, margina tumilo cyaneo, - Lichen cyunefuens Perfon Ut. Ann. St, I1. - Verrucaria cyanefeens Hoffm. Deut. Fl. margine lcutellarua nigro. Obf An a L. Hageni ditinatis? Patellaria cyanefcens. caud, Fagi。

+ L. detritus - cruftaceus albido cinereus; fcutellis difformibus detritis a crufta marginatis, Verrucaria detrita Hoffm. Deut. Fl.

Obf, Scutellæ jun, pallidæ demum emerlæ ru* fefcentes fubfulcæ maryinatz.

Patcllaria detrita. cort, arb.

+ L. flavofucus - cruftaceus obicure flavus granulofus; fcutellis demum convexis irregularibus rufefcenti fufcis, - Lichen flavofuicus Schrad. Spicil. Fl. Germ. Gmel. Sylt, Linn, - Verru. caria flavofulca Hoffm. Deut. Fl.

Pacellaria flavofus ca a faxa aren. $\quad$ L。 
+ Lo fruffulofus - cruftaceus rimofisfimus ex albido \& nigro variegatus; Icutellis fuicis demum convexis fufconigris - Lichen fruftulolus Dicks. Cr. Britt. 3. T. 8. f. 10.

Obf. Scutella juniones dilutisfime fulca plana albido marginatæ,

Patellaria finfulofos. sup.

f L. minatisfmoms - cruftaceus tenuis glaber alboflavefcens; icutellis planis minutisfimis fufcis, mar. gine albo - Lichen minutisfimus Hagen Hif. Lich. T. 1. f. 7. Hoffm. Enum. Gmel. Syit. Linna An id. cum L. vario? v. f. 1.

Patellaria minutisfirsa. tunce arbor.

* $\%$.

* L. incarnatus - cruftaceus granulatus fufcus; fcur. tellis incarnatis pruinofs demum convexis flexuofis. - Lichen incarnatus Thwoberg. Fl. Cap. \& Ic ined.

Patellaria incarnata. in arenof。ad Cap. b. Sp.

* 8 .

† L. crenulatus - cruftaceus rugofus niger; fcutellis cinereis albido narginatis. - Lichen crenulatus Dicks. Cr. Br. 3. T. 9. f. I. Patellaric crenulata. rup.

* 9 .

† L. Dicksonii - cruffaceus ferrugineo ochraceus; fcutellis elevatis atrocærulefcentibus pruinofis, margine obufo. - Lichen Dickfonii Gmel. Sylt. Lisin, - Lichen cxilus Dicks. Crypt, Br, 2. T. 6. f. 6 .

Patellarıa Dickfonii. rup. 
+ L. pruinofus - cruftaceus tenuisfimus ferrugineo rufefcens; fcutellis difformibus pruănofis demum convexis atro cærulefcentibus, - Lichen prui natus Dicks. Cr. Br. T. 9. f. 4.

Patellaria pruinofa. laxa.

+ Lo dendriticus - cruftaceus cinereo nigrefcens ansbitu laciniato-fimbriato; fcutellis demum convexis concoloribus. - Verrucaria dendritica Hoffm. Pl. Lich. T, 19. f 4. - Plora dendritica Hoffm. Deut. Fl.

Obi. Crufte expanfio radiofa inftar L. Swartziii. Patellaria dendratica. Quartz. Granit。

\section{* 10 .}

$+L_{*}$ argillacers - cruftaceus obfcure luteus; tuberculis extus nigris intus ex rubro fulvis. Lichen argillaceus Bellardi App. Fl, Pedem. Patellaria argillacea. ters. argill.

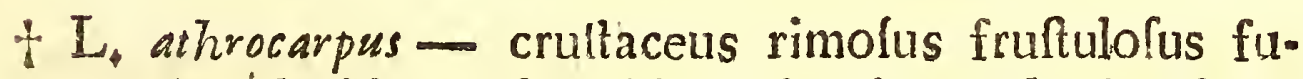
Ico' luridus; fcutellis minutis confertis nigris expanfis marginatis. - Verrucaria fafciculata Hoffm. Deut. Fl.

Patellaria athrocarpa. rup.

+ L. efcharoides - cruftaceus granulofus craslus co. hærens cervinus inzquabilis; feutellis convexis turbinatis nigricantibus. - Lichen efcharoides Ehrh. Crypt. exf. - Verrucaria efcharoides Hoffm. Deut, $F$ l。 crufta crasfa lemipollicari interne nigricante hinc inde e cinereo ferruginea. Patellaris efcharoides. in turfolis.

* L. gosfypinzs - cruftaceus mollis tomentofus candidus, fcutellis planis nigris, margine albido. Lichen goslypinus Swartz. Prodr.Gmel. Sylt. Lin. Patellaria gos/ypina. fupra mufcos Ind. occid. 


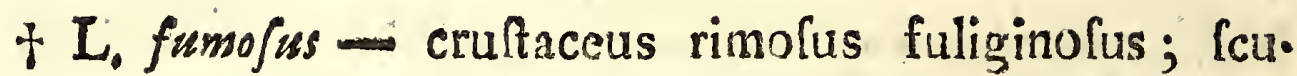
tellis immerlis nigris convexis prominulis mar. ginatis. - Verrucaria fumola Hoffm. Deut. Fl. \& Pl. Lich. T. 49, f. 2.

Patellaria fumofa. faxa aren.

+ L. hemipharicus - crultacens tenuis lævis lutelcens; fcutellis immerlis convexis atris politis, - $\mathrm{Li}$. chen hemisphæricus Hagen. Hift. Lich, Hoffm。 Enum, Gmel. Sylt, Linn. Patellaria hemispherica. cort, arb.

+ L, pilslaris - crutaceus cinereo albidus; fcutellis dem 1 n hemisoharicis nigris. - Lichen pilularis Divies Aet Sor. Linn. 2. T. 28, f. I. Hulu; loci forte etiam eft: Verrucaria pilularis Hoffm. Deut, Fl. alba, fcutellis nigris convexis hemisphericis immarginatis,

Patellaria pilularis.

+ L. pericleus - cruftaceus albidus fparfus; fcutellis nigris convexis albo marginatis demum hemifphæricis, - Lichen abietinus Ehrh. Cr. Exf, Verrucaria abietina Hoffm. Deut. Fl. Patellaria periclea. cort. Pini Abiet.

t L. fimplex - ecruitaceus; fcutellis difarmibus plicato rugofis atris. - Lichen fimplex Davies Act. Soc. Linn. 2 T, 28. f. 2. Patellaria fimplex. iaxa.

+ L. Sanguineodter - cruftaceus albidovirefcens; fcutellis hemisphericis inmaruinatis faturate fan. guineis demum atris - Lichen fanguineoater Wulf. ap. Jacqu, Coll. 3. p. I17. Gmel. Syft. Linn, - Forte etiam h. l. Verrucaria atrofanguinea Hoffm, Deut $\mathrm{Fl}$, crulta inconfpicua, fcutellis 
tellis minutis planis atrofanguineis nigro mar. ginatis.

Patellaria Janguineoatra. terr. argill.

+ L. epibryon - cruflaceus pulverulentus impalpabi. lis niveus; fcutellis atropurpurafcentibns, margine albo - Lichen hypnorum $W$ ulf. ap. Jacqu. Coll. 4. T. 7. f. 2. Patellaria epibryon. múcis inftrat.

+ L. Scytropus - cruftaceus rimofus fubfarinaccus grileofuicus; fcutellis lentiformibus imnarginatis atris. - Lichen fufcoater Wulf. ap. Jacqu. Coll. 2. T. 14. f. 3. 4:- Verrucaria fulcoatra Hoffm. Pl. Lich. T. 54. f. 1, \& Ejusd, Deut, Fl, cinereofuica rimola, fcutellis aigris deprestis contiguis. Ab hac vix differt: Verrucaria cinereoatra Ejusd. Deut Fl. cinereo grifeo-albida rimofa truftulofa, fcutcllis nigris depresfis fubcon. tiguis.

Obf. Species a L. fufcoatro Linn. \& noftroval. de dillincti.

Patellaria fcytropa, faxa. arb.

t L. viridiater - crufiaceus papillofus pallidus e flavovirefcens; fcutellis atris. - Lichen viridiater Wulf. ap. Jacqu, Coll. 2. p. I86. Gmel,Syft, Linn. Patellaria viridiatra.

† I. varians - cruftaceus albus; fcutellis atris nitidis margine albo. - Lichen varians Davies Act. Soc. Linn. 2.

ObC, Pro diverfa atate color \& fructura rece. ptaculorum nutatur. cfr. Davies 1, c, - forte affinis L. impoliti?

Patellaria varians. faxa.

t L. coracinus - cruftaceus rimofus niger; fcutellis con- 
fertis depresfis atris. - Verrucaria coracina Hoffm. Deut. Fl. - Varietas hujus junior efi: Lichen atrócinereus Dicks. Cr. Br. 3. T.9. f. 2. fcutellis cimereo marginatis.

Patellaria coracina. faxa. rup.

+ I. termizalis - cruftaceus niger, fcutellis convexis conculoribus. - Lichen terminalis Flo Dan. T. 879, f. I. Retz Fr, 2.

Patellaria zermanaliso faxa rup.

* L. pallidoniger - cruftaceus pulverulentus pallido ferrugineus; fcutellis fubelevatis planis atris Lichen pallidoniger Thurb. Fl. Cap. ined.

Patellaria pallidonigra. in argillor. Cap. b. Sp.

* L.? hymenius - crufiaceus pallidovitefcens levis fubmembranacens; fcutellis nigris claufis, demum erumpentibus, difco planiulculo irregulari. - Lichen pertufus Wulf. ap. Jacqu. Coll, 2. T. 13. f. 3. Schrod. Spicil. T. I. f. S. Pazellara? hymenia. cor, arb.

NotA. Lichen betulinus, Firds. Fl, Angl. Hoffm. E. num. Lich. Gmel. Syle. Linn; forte non exiflit. Lichen colliculofus Hoffin. Enum. Lich. T. 2. f. 2. Gmel.Syf.Linn. - Sphxial leucocephala Ehrh. e Lichenum numero excludendus, $\mathcal{O}^{\circ}$ potius Spheria generis eft ob recepracula intus gelatinofa, quamvis bafis tenuisfinae cruflacea.

Lichen rolaceus Retzii Prodr. Fl. Scand. 2. Bel. lardi. Fl. Dan. T. 825. eft Iphæria nivea Hoffm. nec Lichen.

Patellarie mihi audiune plurimi Lichenes le: pro/z, tam foutellati, quam tuberculati Linnai of Auctorum. Hi fere omnes Crufle of Receptaculorum forma a 
Je invicem parum difcrepant. Pro diverfa atalis ratio. ne illa uel cenuis wel crassa, jam orbicularis, jam absque determinata figura expanfa, indolcm foliaceam nun. quam ofterdens, levis aut incqualus; homem etram figura varabion non minori obmaxid eff. Ab infantia receptacula plus winusue cancave, iemporis decin fu (exceptio rarisfina) tandem applanata es denigue convexa, insmo fere globofa reperizutur. Sic nota quadam confanti di-

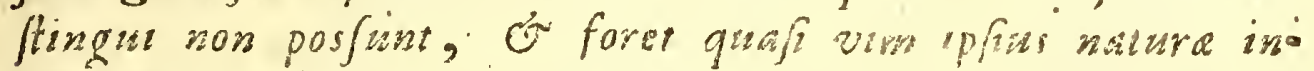
fringere, fi fub diverfo Tribu collocati, Joutellaci nempe Oo tuberculati. foc dicii Lichenes leprof Jeparation proponerentur.

\section{Tribus 7.}

\section{Botomyces Suit. Ko Kóllafyar.}

Cruffa mollior, granulofa difporfa inæqualis. Receptacula: Tubercula rotundata lavia xqualia fungiforma, in bacillis fimplicibus folidis erectis mollioribus crasfiufculis terminalia.

I SI. LICHEN ericetorum _ cruftaceus granulatis al. bicans; bacilis brevisumis obconicis; tuber culis fubglobolis carneis fimplicibus.

Boeamyces. ericetorum. Svet. Healaf.

Lichen ericetorum, Scop. Liljebl. Gmel. Sylt. Linn, var. Atipit, Schreb. Spicil, Web. Pollich. Leers. Huds. Lightf: Mattufch. Hag. Weis. Neck. Meth. Allion. Reich, Fl. Gunn,Roth. Tent, a. Wither. Scholl.

Lichen boeomyces Ehrh. Linn. Suppl. Retz. Pr. 2. Hoffm. Enum. Schrank. prim. \& Fl. Bav.

Boeomyces roleus. Perfoon Uft, Ann. St, 7. Tubercularia ericetorum $W i g g$, Primit, E

Icon, 
Icon, Fl. Dan. T. 1003 f. 2, Hoffm, Enum. Lich. T. צ..3 Dollen. Hift. Nutc.1.14 f.1. Habitat ad terain angllolam macram in juniperitis \& lyivis acerolis.

52. IICHEN bysfoide - curtacers rugofus pulverulentis glatico verefcers, bacillis cylindraceis; tulerculis tu giforn ibus gloneratis ufofulcis. Enconyces bysondes. Surt Supplaf.

Luchen. bysoides Limn, Leers, Buds. Web. Lighef. Wither. Comel, sigh Lino

Lichen furedonis Scop. Reljebl. Web. Hoffm. En. Rorh. Ienito Bollarde, Lumnuz. Crmet. Eyte Linit.

Liches furstorys? Sinrank Centur; bot. Lmo P. 64.

Lithen ericetoum var, \& Weis, var, fungi. formis Polloch.

Enconyes and this Perfoen Uft. Ann. St. 7 . Tubcucularia funeforns $W^{\prime}$ igg. prinit.

Cholonia fungitom ans sthrud. Epicil H. Germ. Icon. Hoffm. Fium Lith. T. 8. f. 2. Fubitat al termm glatcofan \& parietes rimo. fos rupium.

153. I.ICHen rufus - crufaceus teruis pulverulentos virefens; bacillis brevisfinis, tuberculis tomulis fupra plan is fufcis.

Beromgces rufus. Svet, svamplaf.

Lichen rufus Huds. Swartz Act. Upf. 4.Retz. Pr. 2. Wither. Emel. Syft. Inn. Habitatin locis arenofis, aggerum fosfis \& ericetis.

* L. fungoides - cruffacens candidus; bacillis lon* ${ }^{2}$ gionibus cylindraceis; tuberculis difformibus fubglo. 
fubglobofis cameis finplicibus - Lichen funguiles Swartz Protr. Gmel. Sylt. Linn. Boeamyses fungoides, glureos, imont. Ind, Occ.

t I. peltifer-crultaceus nodublus agnofe-virefcens; bacillis albis; tubarculis phardois frounbilicutis dilute fulcis. - Eicher pllifer Wnlf. ap. Jacqu, Coll 3, - Obl, L, bysioilis proninquis. Boemyces peltufer:

t L. Sabuletorum - cruftaceus virefcens; bacillis bre.

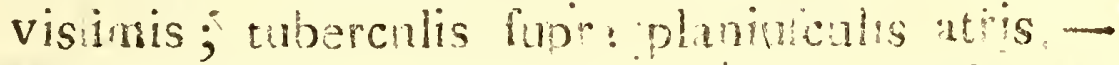
Lichen Thbulatoram Sarucb. Spich. Hafo B. num, Lich. Facq'. Coll, 3, T. 2, f. I. Rosh. Tent, Lumniz: Grael S.yit. Linn.

Bocomyces? fabulecorum. agg. fabil. mire argill.

\section{Tribus 8}

\section{Calicium Suzt. Kapplatyar.}

Crufta tenuior ragofa vel etism pulverulenta irregulariter eff.ta.

Receptacula: Tubercasla lentiformin, rarius fubghobofa, luberola, fupra: difico elevato Roccilofo ex pulverilen. (1); in baillis fimplicibus rigidionibus folids, folitaria, terminalia.

154. Lichen clavellus - crufacens mugulolus aho. cinerafcens; bacillis bai crasforibus, tubarculisque lenticnlaribus nigers lubtus cinereis. Calecium clavollus. Svet. Nibblaf.

Lichen Ipherocephal is var, b. lichenoides Swartz, Act. Upl. 4: var. a. Web.Roth Tent. Mucor lichenóides Linn. Hoffe. Lightf. Gunn. Scholl. Wither.

Cali. 


\section{$-84 .-1$}

Calicium falicinum perfoon. Uft. Ann, St. 7 . Stemonitis lichenoides Gr:el. Syft, Linn.

Trichia denticularis Hoffa. Crypt.

Embolus fepulduralis Batsch. Fung.

Icon, Perfoon. Unt. Ann, d. Bot. St. 7, T. 3. f.

3. a. b. c. Hoffm. Veg. Cr. T.4. f. 3. Bat $/ c h$.

Elench. Fung, 1. f. 133. Dillen. Hitt, Mufc.

T. 14. f. 3 .

Habitat ad cortices antiçns arborum.

Obf. Variat crufta partum virefcente. Tubereula

in initio veio tetia, detn interdum pulvere ferrugineo fupra efflurefcentia.

155. Lichen Spharocephalas - cruftaceus tenuisfmus cinerafcens; bacillis cylindraceis tuberculisque lubglobols nigitis.

Calscium pharoceptialum. Svet. Kardiaf.

Lichen fpliarocephalus Swartz. Act. Upf. 4.

Retz. Pr. 2, Liljebl. Roth. Tent. var. B Web. Mucor fphirocephalus Linn. Hoffb. Lightf.

Gumn. Scholl. Mattufcka.

Stemónitis phærocephala Gmel. Syft. Linn. Trichia Iphzrocephala Hoffin. Veg. Crypt. Icon. Hiofm. Veg. Cr. 2 T. 4. f. 2. Habitat in lignis diutius aëri éxpofitis. Obf. Differt a L. claveillo magnitudine multotics minori, crufta vix ulla, tuberculis fere turbina. wis, difco vallis atris hirto, fubbus nudis. Bacilla conferms fma rigida, omna plerumque ad 26 num latus fese vergentia.

156. LICHen peresellus - cruftaceus tenuis albus; bacillis filiformibus pallidis, tuberculis fubglobolis albo incarnatis.

Calicium peronellum. Svet. Finlaf.

Habitat ad ligna fubputrida in arborum cavis. Obf. L. fphrecophalo minor fed bacilla longiora, 


\section{$-85 \cdot-$}

rarius Sparfa. Tubercula inprimis incarnato pruinoja.

157. LICHEN cantherellus - cruftaceus tenuis pulverulento fubtomentofus albidus, bacillis cylin. dricis lutefcentibus, tuberculis demum lenticuluribus albis.

Caliciusn cantherellum. Svet. Trifslaf.

Cislicium pallidum Perjoon, Uft. Ann. St, 7 . Stemonitis nivea Gmel. Syft. Linn.

Trichia flava? Wither. Arr.

Trichia nivea Haffro. Veg. Crypt.

Icon, Persoon Uti. Annal, d. Bot. St. 7. T. 3. f. I. Hoffm. Veg. Cr. T. 4. f. I.

Habitat ad corticem Fraxini, Pyri \&c. ?

158. Lichen hyperellus. - cruftaceus rimofo rugulofus areolatus glaber flavovirercens; bacillis lis crastis cylindraceis, tuberculisque demum lenticularibus nigropiceis.

Calicium hypereilum. Svet. Spiklaf.

Habirat in cortice Pini Abietis.

Obf. Forte olim cum L. clavello, licet falfo, cans furus.

I59. LICHEN corynellus - cruftaceus pulverulentus flavovirefcens; bacillis brevioribus, tuberculis. que fubglobolis demum lenticularibus atris.

Calicum corynellum. Svet. Nagellaf.

Habitat in faxis.

Obf. Craffa mollis crasfa fulphurea pallida inter. dism etiam albefcens.

Varietas hujus, crafia fulva, granulo o: floc. coja, ad ligna femiputrida runcorum Fraxini aliquando occurrit; An diftincta fpecies?

I60. LICHEN acicularis - cruftaceus pulverulentus nalli- 


\section{$-86$.}

pallidus; bacillis teretibus brevioribus, tuherculis fibolobolis fulvis.

Calicinm ariculare. Svet. Nallaf.

Lichen fulvus Swurtz. Act. Upl. 4. Retz, Pr. 2. Liljebl.

Iichen Inlnhureus Retz. Ad. Ac. Svec 1769.

Mucor fulvus Linn, Svec. Hoffb.

Stemoni is fulva Gmel. Syft. Linn.

Trichia folva. Wither. Arr.

Habitat ad terram in fylvis.

16I. Lichev capitatur - crultacens pulverulentus vi. rilify ayus; becillis elongatis filiformibus, tu. berculisqua fubglobolis pruinofis fulvis demum fulcefcutibis.

Calıcium capitatum. Svet. Klotlaf.

Lichen capitatus schreb. Spicilo Roth. Tent. Gmel. Syt. Linn.

Lichen f'urf iracsus Swartz. Act. Tpf: 4.

Iichen mlobularis Retz. Prodr. Fl. Scand. 2.

Lichen fulphuras I.ljebl. Sv. Fl.

Mucor furfuraceus Linn. Hoffb. Leers. Scholl.

Stemonitis furfurace Gmel, Sylt. Limn。

Trichia furfuracea Wirhow. Arr.

Finbolis furfuracen Batch Fune.

Icon. Batch. Flench. Fung, f. 178. Habitat in terra ad radices arborum.

* L. helopherus - ecruftaceus; bacillis filiformibus confertis, uberculisque fubglobolis glabris

- nigris - Mucor clavatus Linn. Suppl. - An hujus seneris?

Calicizsm helopherum. in fol. Palmar. Cap. b. Sp.

+ L. lygodes - cruitaceus glebulofus viridis: bacillis elon- 


\section{$-8 \%$}

elongatis filiformbus tlexilibus, tuberculis!nigris. - Calicium viride Perjoon. Un, Annod. hot. St. 7.

Cáliciuri lygodes. arb.

NotA. Habitu of frutura partium frublificationis $P$ ao tim dignofcumer calicia, es parkas abefe, gum crea - deve propraum ex his genus fuo jure corfirui debere.

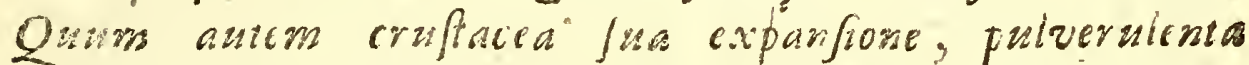

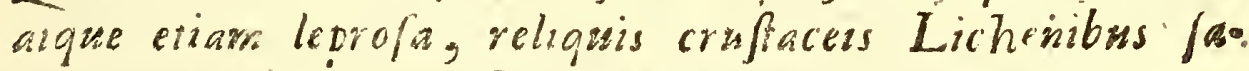
tis affines fint, inter hos jam enumerare malui.

\section{Tribus 9.}

\section{ISIDIUM Svet. CORALLAFVAR.}

Cruffa folida fuborbiculeris \& difformis crasfiufcula; $r$ a mulis coralloideis fimplicibus ramofisque compofita vel faltem ad luperficiem hos producens,

Receptacula: Tubcrcula fubglobora ad apices ramulo. rum fisflia.

162. Lichen corallinus - crufaceus cincreus ramuJofus, ramulis foliclis. cylindricis fafciculatis confertistimis; tuberculis globolis concoloribus,

Ifidium corallinum. Svet, Spródlaf.

Lichen corallinus, Linn. Retz. Pr, 2. Liljebl. Huds. Web. Pollich, Lightf. Wulf. Hoffm. Esum. Foth. Tent. Willars. Bellards: Wuther. Gwel. Sylt, Linn.

Stcrencaulon corallinum Schrad. Spicil. Fl. Germ.

Stereocaulon corallina, Hoffm, Deut, Fl.

Icon: 
Icon. Jacgn. Coll. 2. T. I3, f: 2, Hoffm. Enum: Lich. T. 4. f. 2.

Habitat in tixis.

Obl. Scutelle globofe atre Sparfa, de quibus mentio

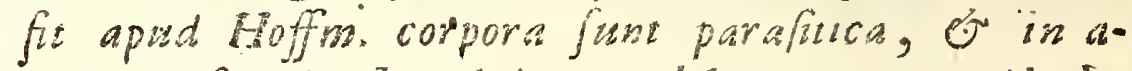
liis crufaceis haud raro objervanur; $v . \%$. L. pertufo, L, globifero o q. T. r. - Icon his. jus Lichenis bona defideratur.

163. Lichen Wefringii - cruftaceus rimolus, areo. lis angulotus, pallidus ramulofus, ramulis 'foldis cylindricis falciculatis faftigiatis confertisfimis; tuberculis globolis fulcis.

Ifraitam Wegringii. Svet. Wepringslaf.

Eichen Weitringii Achar. Act. Ac, Sc, Svec. I794.

Lichen pleudocorallinus wefring. Act. Ac.

Sc, Svec, 179:-Retz. Pr. 2: Liljebl.

Icon. Tab. nofra. 2. f. 2. \& Nov. Act. Ac. Sc. Svec. T. X.V. T. 6. f. I.

Habitat ad rupes.

Ob?. In hajos paricer ac Is corallini crufta, hic illic corpora hemispherica fparfa oceurrunt ex meris ramalis aggregatis confirulia. - Colore, cruAte forma of tuberculis furfis a pracedente certisfime diffinctus.

16. Lrchen papillaria - cruftaceus granulofus cinerens ramulofus; ramulis difcretis erectis fubfimplicibus brevibus fiftulofis ventricofis albis, tuberculis globolis nigrofufcis.

Ifidisus papillaria. Svet. Pattlaf.

Lichen papillaria Ehrh. Beytr. Dicks. Wither. Gmel. Sylt. Linn.

Lichen madræporiformis Wulf, ap. Jacqu, Gmel. Sylt, Linn.

Lichen 
Lichen madreporoides Rutfr. Dislert.

Lichen fragilis a) minimus. Web. Spicil.

Cladonia papillaria. Hoffm. Deuc. Fl.

Icon. Facqu. Coll, $3 . \mathrm{T}, 3, \mathrm{f}, 2$.

Habitut lupra terram.

Obl. Ramuli ad apices attenuati, ctiam fis $\sqrt{2}$ \& fubdigitati obfervantur, atgue fub hac forma $a$ pud Hoffm. appellasur:

Cladona molariformis, Ejusd. Deut. Fl, Dillen。, inusco T. 16, f. 28 .

Ob ramulos falsulojos inerdam ramolos Cladowi is affinss.

165. Lichen daciylinus - cruftaceus vermcofus albocineralcers ramulofus; ramis redis, fructiferis cylindricis demum turbinatis folidis fimplicibus a pice rufelcentibus. Ifidizm dactylinum. Svet. Spenlaf. Habitat in terra macra alpeftri.

Obf. Quemadmodum in Spher ophor is apices ra. mulor sars frudtificantium pasfim rumpunur es tuberculum, glande pesis intra proputizsm pro parte abfcondita of Jupra rimula notate, hand abfimble, oftendiszt.

I66. LICHEN gonatodes - cruftaceus albocinerafcens ramulolus, ramulis divaricatis lobatoincilis torulofis tortuofis difformibus folidis obtulis. Ifidium gonatodes. Svet. Knaggellaf. Stereocaulon madreporiforme? Hoffm. Deut, $\mathrm{Fl}_{\text {. }}$ Habitat ad terram.

Obf, Tubercula nulla adhuc detecta.

167. LrCHeN cereolus - cruftaceus granulofus cine. reus, ramis fparis erectis folidis rigidis fimplicibus pulverulentis, tuberculis mi. nutis hemisphricis nigrofulcis, 
Ifidium cereolus. Svet. Facklelaf.

Habitat ad laxa durislima arenaria.

Obf. Singulares onnino Luthen, candele l. cereoli extuncti Speciems pro fe ferens. An crufta hujus propria fit aut alsena. non certo confat, natm in ea etiam tubercula alia majora Jerfilia affixia femel vidi.

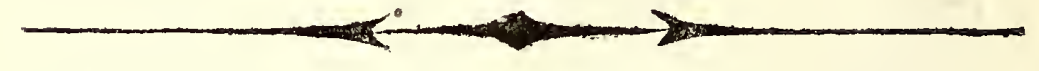

f L, horophthalmin - cruftaceus albus fungolo- papillofus ramulofusque; tuberculis nigris tesfilibus $\&$ in ramulis terminalibus. - Lichen oculatus Dicks, Cr. Britt. 2. T. 6. f, 3. Wi. ther. Gmel. Syft Linn.

Ifidium herophihalmum. rup. saxa.

* L, tabularis - cruftaceus cinereo pallidus ramulo. fus, ramis divaricatis extrorfum crasfioribus; tuberculis convexis nigris glabris terminalibus. - Lichen tabularis Thunb. Fl. Cap. ined. c, fo - Lichen verrucofus Linn. - Li. chen verruciger Grrel. Syft. Linn. Ifridum tabulare. mont. Cap. b. Sp.

$\div$ L. defraudans - cruftaceus ramulofus, ramulis confertisfimis faftigiatis, extremitatibns contextis in cruftam planam nigram; tuberculis viridibus fupra aggregatis, - Lichen defraudans Olaffen. Island. App.

Obi. Nefcio quo pacto fieri potuit ut hunc a L. corallino adeo diftirctum quafi fynony. mon ejusdem allegerint Auctores. Ifidiums defraudans, rup. 


\section{- 91. - \\ Tribus ro.}

\section{Psoroma Svet. Spånlafyar.}

Crufta rolida irregularis effigurata, foliolis leprofis diftinctis fqumatim incum rentibus conpofica aut tecta.

Receptacula: Siutella (parlx marginatx planx, demum convexx.

168. Liches microphyllus - cruftaceus imbricatus, foliolis minu is ims creanlatis cinereofulcis ; fcutellis demum convexis fufcis foliis mixtis cinct isque.

PSoroma microphvlla. Svet. Smäbladslaf.

Lich 'n microphyllus Swartz. Act. Upf. 4. Wefr. Act Sc. Svec, 179 r. Liljebl. Retz.

Pr. 2. Schrad. Spicil. Gmel. Sylt. Linn. Achar. Act Sc. Svec, 1795.

Plora microphylla Hoff m. Deut. Fl.

Icon, Achar. Nov, Act, Ac. Sc. Svec, T. XVI.

T. 5.f 3. Schrad Spicil. Fl Germ, T. I. f. 4. Habitat ad latera montium demisfa \& truncos arborum.

Obf, Quamvis lpecie non differunt tamen ut vorietates confiderandi heic veniunt:

Lichen carnofus Dicks. Cr. Britt. T. 6. f. 7. Wither. Arr. Foliis confertis/zmis erectinfoulis rotundatis virentibus margine crenulato albo pulveralento, foutellis crassis elevatis rufis. h. l. eßf:

Pfora carnofa Hoffm. Detut. Fl. \&

Pfora grifea Hoffm. l. c. fouellis nigris a crus fta marginatis margine crenulato demum convexis.

Stereocaulon corallinoides Hoffm, l. c. cruftaceus atrocinereus ramulofus, foutellis convexis nigris.

In- 
In corice longe lateque expanditur foliorum primor. dialum lacinisis, forte a nullo adhuc ob/ervatis, alsguando in ramulos crralloideos confertisfimos abeuntibus, (Stereoc, corallinoides Hoffm。 ) unde factes omnino Ifidii. Demonlerat hec metamorphofas neque inter Ifidia neque Stere. ocaula collocari debere. Scutelle jam lusefceno

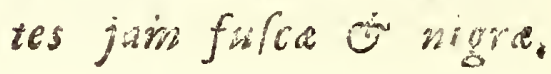

I69. LiCuen niger - cruftaceus fuliginofo niger, foliols imbricatis lobatis minutishais in cento tenuishe fubramiofis, cutellis fparlis concevis demum planis atris.

Pforona nigra. Svet Suartaf.

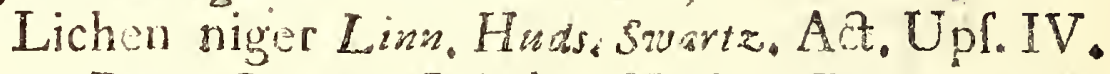
Retz. Pr. 2. Liijebl. Hoffm. Enum, Roth. Tent.

Lichen antiquitatis Reich. Fl. Frankf. Lichen ater Schrank. Nl. bav. Lichen fufcoater? Pollich Pl. Palat. Colloma nigrum Hoffri, Dent. FJ. Icon, Hoffm. Enum. Lichen. T. 3. f. 6. Habitat lupra lapides inprimis calcarios. Obf. Orbiculation in crespe formam expanditur. In perfectis fpeciminibus folia ad extrowum ambitums faxo adpresfa, minsia, difcreia, quali palmata, pulposaque confpiciunsur, o ipfe margo Lio chenis, ut in pracedenti, colore carulefcenis. nigro cingitur. Siccus aguam citisfome bibulus fubgclatinofus evadit, wit etiam in aliis quibuse dam cruftaceis obfervare facile eft. Conformatio docet nec Collema nec Patellariam effe.

170. LICHEN candelarizus - cruftaceus imbricatus tlavus, foliolis congeftis laciniato laceris; fcu. tellis demum convexis Juteis. Pforoma earadelaria. Svet, Ljuslaf, 
Lichen candelarius Linn. Retz, Pr, 2. Liljebl. Hoffb. Scop. Huds. Web. var, a. Lighef. Fl. Scot. Weis. Schreb, Spicil, Mattufch. Hag. Crantz. Gorter. Leers.- Pollich. Leysfo Allion. Eloffin. Enum. Reich. Fl, Roth. Tent. Mull. Gmelin. Tybing. Scholl. Mohr, Lumnitz. Wither,Gmel.Syit, Linn。

Lichen fulvus Neck. Meth.

Lichen flavicans Gmel. Syit. Linn.

Lichen Linkii? Gmel. Syft. Linn.

Lichen ffavelcens? Link. Ann.

Lichen concolor Dicks. Rufer.

Lichen polycarpus? Ehrh. Cr. exf.

Lobaria polycarpa? Hoffin. Deut. Fl.

Lobaria concolor Horfm. Deut. Fl.

Lobaria candelaris o I. fulva. Hoffmo ibid.

Placodium candelarium Wigg. Prim.

Icon. Hoffm. Enum. Lich, T. 9. f. 3. Dillen.

Hit. Mulc. T. 18. f. I8. B.

Habitat in faxis, muris, parietibus, arborum. oue truncis.

Obr. Fo:ma \& color fcutellarum a diverfis loci of etatis mutationibus egregie variant. Crufta enime jam concolores, jam-fulva, aurantiaca of ma. gis rufe/centes funt, figura plane, marginate, convexe \& immarginata; fparfa alque conferte. Folia perexigua congefta imbricata, margine interdum cripa, tenuisfime ramulola, hand raro etiam evanida of cruftam tantsum pulverslentam exhibente colore flava, fulva o s aurantiaca. Hinc Species multa Auctorum fictitic reducenda.

171. LICHEN hypnorum - cruftaceus imbricatus viridifufcus, foliolis laciniato incifis margine erolo granulatis; fcutellis obfcure rufis margine crulta concolore.

Pforoma hyprorum. Svet, Vaggmofslaf. 
Lichen hypnorum Oed. Fl. Dan, Retz. Pr. 2. Wither. Dicks. (excl. fynom.) Plora hypnorum. Hoffm. Deut. Fl. Icon. Fl. Dan. T. 956.

Hahitat af terra:n fub tratis \& intermixtis mufcis \& fupra ens.

Obf Varietas hujue Pecundum Cl. Hoffm. erit:

Pfora deaurart. Loponfo granulo/o-foliolofa flave'cens, lcutelis rufeccentibus margine crispo. Eirusd Deut Fl.

172. Lrenes mefrularis - cruftaceus imbricatus fufoo cxius cineren puinofis, foliolis diformibus fublobatis convexis turgidis adfcendenibus: Foutellis demum convexis nigris. Pforom veficularis. Sver. Bithblelaf. Lichen graniforais Ehrh. Crypt, exf.

Li hen grifass Gmel. Syt. Lina.

Iichen polymorphus La Touretze Tugd.

Lichen cær!lonnigricans Lightf. Liljebl. Hoffmo

Enum. Gmel. Sytt, Linn.

Patellaria veficularis Hoffm. Pl, Lich.

Pfora veficularis Hoffm. Deut. Fl.

Verrucaria grifea Wild. Bot. Mag.

Icon, Hoffm. Pl. Lich. T. 32. f. 3. Wild. Bot.

Magaz. 4. T. 2 f. 1.

Lichen candidus Wcberi, Retz, Pr. 2. Ehrh.

Bellardi, Roth. Tent. Gmel. Sy?. Linn.

Lichen elbingarodenfis Comment. Lipf. V. 29. Patellaria candida Hofm. Pl. Lich.

Pfora candida $\mathrm{Hoffm}$ Deut. Fl.

Icon. Fl. Dan, T, 1064. f. I. Hoffm. Pl, Lich. T. 33, f. 2.

Habitat ad rupes, deffructis mufcis inftratus. Obf. Species Dolymorpha. In ireniore Lichene

(f. L. candito) folia magis lobata funt, $\sigma^{\circ}$ pruina candida tegitur tota Juperficies; hac ve- 
ro deterfa folia fufca o cum foutellis etions nigra condurt.

173. LiCHEN luridris - cruftaceus imbricatus, foliolis orbiculatis crenatis, viridi fúcis fubtus pallidioribus; fcutellis demumi convexis nigris. Pforoma lurida. Svet, Mörkiaf.

Lichen lundus Swartz. Act. Upf. 4. Weftr. Act, Sc. Svec. 1791. Retz, Pr. 2.2.ljo hl. Dicks, Schrad. Spicil. Leers. Wither. Grel. Syit. Linn. Achar. Act, Sc, Svec, 1795. Icon. Achar. Nov, Act. Ac. Sc. Sver. T. XVI.

T. 5. f. 2. Fl. Dan. T. 30. f. 134. Nich. Gen. T. 54. f. 4.

Habitat ad rupes fupra terram fubfiratis in. terdum nuficis.

Obl. Varietates hujus forte Sunt:"

Lichen fquamatus Dicks. Grnel, Syft. Linnai qui:

Lichen fquamofus? Willars. Bellardı.

Lichen pulpofus Leers. Gmel. Sylt. Linn. Go

Plora fquamata Hoffm. Dent $\mathrm{Fl}$. foliis majoribus finuatis glanco viriaibus. Dillen. $H_{1} / l_{\text {. }}$ T. 30. f. 135. atque:

Pfora turbinata Hoffm. Deut, Fl. foutellis turbinatis rugulojis.

174. LICHEN fcalaris - cruftaceus imbricatus, foliolis reniformibus erectiufculis difcretis pallidic, fubtus margineque pulverulentis; fcutellis planis nigris.

Pforoma foalaris. Svet, Flarnlaf.

Lichen fcalaris Achar. Act. Sc, Svec, 1795. Hiora oftreata Hoffm. Deut. Fl.

Icon, Achar. Nov, Act. Ac, Sc. Svec. T. XVI, T. 5 . f. I.

Habitat in cortice antiqua truncorum Pini fylv: \& ligno Juniperi exarido vetufto.

Obf. 
Ob!. Foliorum incipientium Scyphiferorum faciems hic quodammodo pre fe fert. Color virescenti grufeus ad margines quar pallidior. Scutelle ummixte marginsice, rarisfima.

175. Licher decipiens - cruftaceus, foliolis difcretis fubromandis concaviulculis rufofulvis, fubtus margineque albis; fcutellis demum convexis vigris targinalibus.

Pjoroma decipieas. Svet. Falsklaf.

Lichen decipiens Hedw. Ehrh. Dicks. Schrad. Spicil. Wuther. Gmel. Sylt. Linn.

Lichen pezizoides Swartz. At. Upf; 4 , Retz. Pr. 2. Liljebl.

Lichen itellatus Relhan. Cantabr.

Lichen ftellariformis Schrank. Bavar.

Iichen elveioides $W^{\prime}$ ulf. ap. Jacqu.

Lichen elveliformis Gmel. Sylt. Linn. Lichen proteiformis La Tourette Lugd. Lichen pantolpermus Villars. Hitt. 3 . Lichen peltiphyllos Bellarde App. Pedem. Lichen difpermos Villars. Fl. Delph.

Pfora decipiens Hoffm, Pl. Lich, \& Deut Fl. Icon. Hedvig. Strip. Crypt. T. I. f. B. Hoffm.

P]. Lich. T. 43. f. I - 3. Jacgu. Coll. 3. T. 3. f. 3. Villars. Hift. 3. T. 55 .

Habitat in terra nuda montium calcariorum. Obf. Folia juniora Jubeancava, foutellas mentientia.

176. LICHeN teftacens - cruftaceus imbricatus, foliolis ovalibus aggregatis remotisque viridi cinerafcentibus; fcutellis demum convexis rufis marginalibus.

Pforoma teftacea. Svet, Skallaf. Pfora tefiacea Hoffm. Pl. Lich, \& Deut. Fl. Icon. Hoffm. Pl, Lich. T, 22, fo 5. 6 , Habitat in faxis calcareis, 
177. Lichen cartilagineus - cruftaceus imbricalus filbpulvinatus, foliolis lineari laciniatis incifis adicendentibus pallidis; fcutellis planis fulvis, margine albo cienulato.

Pforoma cartilagined. Svet. Brolklaf.

Lichen cartiagineus Swartz. Weftr. Act. Sc.

Svec. 1791. Liljebl. Achar, Act, Sc, Svec,

I795.

Icon. Achar, Nov, Act. Ac. Sc:Sviec, T. XVI.

T. 5. f. 4. Fl. Dan. T. Ioub.

Habitat fupra montes vix humo tectos.

Obf Lichen totus pulvinatus, foutellis in medio confertis, fuborbicularis. Cum L. craslo a Lightfootio injufte confulus.

178. Lichen crasfus - cruftaceus imbricatus fubpulvinatus, foliolis rotundatis lobatis planiufculis undulatis albovirefcentibus; fcutellis rufofulvis.

Pforoma crasfa. Svet, Tjocklaf.

Lichen crasfus Hudfoon. Fl. Angl, Sysartz. ACt, Upf. 4. Weftr. Act. Sc. Sv. 1791. Retz. Pr. 2. Liljebl. Hcffm. Enum. Lich. Schrad. Spicil, Gmel. Syit, Linn.

Lichen cartilagineus Lighif. Fl. Scot, Retz. Pr. 2. Wither.

Lichen laqueatus Wulf. ap. Jacqu, Gmel。 Sylt, Linn.

Lichen cæepitofus Villars. Bellardi. Lobaria laqueata $\mathrm{H}$ offm. Deut. Fl. Icon. Facgu. Coll. 3. T. 5. f. 2. Hoffm. Enum. Lich. T. I9. f. 1. Villars. Delph. 3. T. 55. Michel, Gen, T. 5I. f. 2. Dillen. Hift. Mulc. T. 24. f. 74 .

Habitat in terra ad montes præcipue calcarios. Obf. A nofire disix diffort: 


\section{- 98. -}

Lobaria crastiformis Hoffm. Dent. Fl foltis jubtus of miargine nigricantibus.

+ L. Smithii - cruftaceus imbricatus, folsolis loba. tis virentibus fubtus atlbis; foutellis diformibus thavelcentibus. - Iichen yplaceus $S_{\text {mith }}$. Act. Soc. Linn. 2. - Lichen fragilis Srop. Cam. Michel. Gen. 94. T. 1. f. 1. Plorom swathii. sup.

* L. syprophilus - cruftaceus fubimbricatus ${ }^{\circ}$ foliolis dincretis difornibus crisfis carreis, fcutellis. demum convexis nigris. - Lichen gyplophilns Schred. Spicil. - Lora gyplophila Hoffm. Lietit $\mathrm{H}$.

Obf, An a $L$, decipienti diftincus?

Pforomat sypfophalu. mont, calcar. gype.

t. L. tmadinlus - cruftaceus, foliolis albis lobatis, lobis dethxis tumiclis; fcutellis convexis atris diformibus. - Lichen tunidulus Swith. Act. Soc. Linn. 2.

Pforoma rumidula. tisfur. rup.

† L。 coloun - crufteceus Jobatus crisplis, lobis depresfis albo marginatis; fcutellis uffetcentibus. - Flora cælata Hoffm. Deut, Fl. fub. tus albus.

Pjoroma calata.

$\div$ L. levcophans - cruffaceus imbricatus, foliolis mimutisfimis crenato multitid is glaucofucis, lobis apice incrastatis albofarinaceis; fcutellis planis nigris, margine albo. - Lichen leucophieus Fl. Dan. T. 955. f. 2, Retz. Fr. 2. Dicks. 
Dicks. Cr. Britt. 2. Gmel, Sylt. Lim. Dillen, Hit. Mufc. T. 82. f. 2

Pforoma lescophas. terra rup.

+ L. paradoxus - crultaceus imbricatus fubtesfulatus, foliolis aggregatis convexis unbrino rufof $x$ fcis; fcutellis pruinotis margine albo demum convexis nigris. - Lichen paradoxus Ehr. hart. Cr. Exfo - Hora paradoxat Hoffin. Deut. Fl.

Obf. Folia juniora fcatelliformia murginata, dein convexa fruitinlola.

Pforoma paradoxa.

+ L. gelidiformis - cruitaceus foliolis depresis rimofo angu ois; Ccutellis planis ruhs, margine crufta concolore. - Pfoia gelidiformis Hoffin. Deut, Fl.

Pforoma gelidiformis, mont.

† L. fimilaris - cruftaceus fubimbricatus, follolis agyregatis fruitulo is convexis albidis; fcutellis marquatis concoloribus, - Pfora foni. laris Hof m. Deut. Fl.

Obr. Folia glebuloria etiam ex carneo albida. Pforoma fimilaris. faxa, lign.

* 'L, ribiginofus - cruftaceus imbricatus foliolis gra. nulato lobatis cinereovirefcenibus; fcutellis ruis platis, margine fubcrenulato flexuolo crutia concolore

PJoroma rubiginofa. Afr. Thunberg. Ic. ined.

+ L. chryolewhos - crutuceus imbricatus, foliolis lobatis obta is pallide fulphureis fubtus atro. virtibus; fatellis aureis. - Lichen chryfo. leucos Smith. A t. Soc. Linn. 1. T. 4. 1. 2. Pforoma chryfolescos. rup. alp. t $h_{2}$ 
I. rubinus - cruftaceus fubimbricatus, foliolis pulpolis lobatis crenatis cinereis; fcutellis pallide rubinis, - Squamaria rubina Hoffm. P. Lich. T. $32, f_{*} \mathbf{I}$, Lichen rubinus? Lamark. Villars.

Obf. Maxine afinis L. chryfoleuco. Pforoma rubina. faxof,

- I. Jaxifragus - crulaceus imbricatus? lobatus longistime radicatus cælius; tuberculis rubrls. - Lichen faxifragus Smith. Act. Soc. Linn. 2. Pforoma? faxifraga. fisf. rup.

* $\Upsilon_{\text {。 }}$ torulo/us - cruftaceus imbricatus fubpulvinatus, foliolis lineari laciniatis adfcendentibus cinereis margine granulato torulofis.

Obf. Scutellæ mihi defiderantur. Ob propinquitatem cum L. cartilagineo huc retuli. Pforoma toruloja. Cap. b fp. Thunberg. Ic, ined.

\section{Tribus ri.}

Placodium svet. Kretslafiar.

Cruffa folida orbicularis vix imbricata plana, folii ex: panfi rotundati atque compofiti formam pre fe ferens, Pobata; lobis vel concietis vel tantum in ambitu di-
ftinguendis.

Receptacula: Siztella utplurimum centrales, marginatx planx aut convexx.

I79. LiCHEN circinatus - cruftacens orbicularis rimofus, ambitu radiofo plicato, cinereo cæifcius; fcutellis confertis planis nigrofufcis, margine tenui cinerafcente.

Placodium circinatum, Svet, Kranslaf. 
Lichen circinatus Persoon. Schrad. Spicil.

Lichen fubimbricatus Relhan. Wither. Gmel. Sylt, Linn.

Lichen radiofus Hnffm. En, Lich. Gmel. Sylt; Linn. Achar. Act. Ac, Sc. Svec. I794. " Lobaria radiofa Hoffm. Deut. Fl.

Icon. Hoffm. Enum. Lich. T. 4. f. 5. Relhan。

Canabr. p. 427. tab. Achar. Nov. Act, Ac.

Sc. Svec. T. XV. T. 6. f, 5.

Habitat ad lapides calcarios.

3. LICHEN murorum - cruftaceus orbicularis rï: mofus, a mbitu radiofo plicato, flavovirefcens; fcutellis demum convexis rufoluteis.

Placodium murorum. Svet Murlaf.

Lichen murorum Hoffm. En. Lich. Ruftr. Lumnitz.

Lichen flavefcens Hudf. Retz, Pr. 2. Liljebl. Wither, Roth. Tent, Hoffm. Enum. Oed, Enum.

Lichen candelaris Wulfen, ap. Jacqu.

Lichen candelarius var. $\beta$ Lightf. Fl, Scot, Lichen parietinus Leers. Herborn.

Pfora favicola Hoffm. Pl, Lich.

Lobaria faxicola Hoffm. Deut, Fl.

Icon. Hoffm. Enum. Lich. T, 9 f. 2. Ejusd。

PI Lich. T. 17. f, 3. Jacqu. Coll. 3. T. 6.

f. 1. Dillen. Hiti Mufc. T. 18. f. 18. A. C.

Habitat in muris tegulis \& fupra rupes calcarios. Obf Variat fouteliarum colore magis pallido atgue margene fuhulbido. Scutelle interdum fere abso que omni crufta dipperfa inveniuntur. Totus Lichen per lenium undique pulverulentus ơ ne veftigia quidem loborum relinquens.

Aut hujus vel forte potius sequentis varietas efts: Lichen tegularis Ehrh, cr, exf. qui: 
Lobaria tegularis Hoffm. Deut. Fl. pl. Lich. l. c. f. a. crufia \& foutellis aurantiacis.

181. Lichen elegans - crufaceus fuborbicularis allrantiacus, lineari multilidus; fcuteliis marginatis concoloribus.

Placodium elegans. Svet. Blanklaf.

Lich n elegans Link. Annal. Gmel. Syft. Linn.

Lichen flavelcens Swartz. A\&. Upf. T. IV.

Lichen fulvus Dicks. Crypt. Brit. 3.

Lichen cinnabarinus Bellardi. Gmel. Sylt, Linn.

Lichen miniatus Hoffm. Enum. Lich.

Lobaria miniuta Hoffm. Deut. Fl.

Icon. Dillen. Hit, Mulc. T. 24. f. 68.

Habizat in rupibus prefertin calcariis.

Obi. Colare variat flavefcente, assrantiaco of miniato atque crufta interdusin vix folsofa. Medius quafa inter L. murormm of L. parietinum, at ab utrogue ominino difunctus.

182. Lichen fulgers - crufraceus orbicularis plicato flexuolus lobatus flavefcens; fcutellis de. mum convexis rubris.

Placodium fulgens. Svit. Skimmerlaf.

Lichen fulgens Swariz, Act. Up?. T. IV. Retz, Pr, 2. Gmol. Sylt, Linn.

Lichen citrinus Ehrh. Hedw. Schrad. Gmel. Syit. Linn.

Lichen friabilis Villais. Fl. Delph.

Lichen crasfus var. A. Liljebl. Sv. Fl.

Pfora citrina Hoff n. Pl. Lich, \& Deut. Fl. Icon. Hoffu. Nl. Lich. T. 4\%, f. 2. Hetw, Crypt.

2. T. 20 f, c, Villars. Wil. Delph, T. 55 . Habitat in terra calcaria múcis fepe cinctus.

183. Lichen bratteatus - cru? aceus orbicularis granulofus fubimbricatus margine fublobatus citrinus. - 


\section{$-103 .-$}

Placodium bracteatum. Svet. Bractealiaf. Pfora bracteata Hoffm. Deut. Fl.

Habitat ad terram in montibus calcariis.

Obf. Receptacula igrota. Ob cruftam planam orbicularem $\mathcal{O}$ in ambitu tennusfome lobutam Pla. codit is potzus quam. P foromis adnumerandus.

184. LiCHen canefcens - cruftacens fuborbicularis farinoius glauco candicans, anbitu corrugatolobatus; futellis marginatis demum conve$\mathrm{xis}$ nigris.

Placodium canefcens. Svet. Hvitgrälaf.

Lichen canefcens Dicks. Cr. Britt. I. Wither. Gmel. Syit. Linn.

Lichen canus Gmel. Sygt. Linn.

Lichen incanus Relhan. Cantabr.

Fiora canefcens Hoffm. Deut. Fl.

Icon. Dickfon. P1. Crypt. Brit. 1. T. 2. f. 5. Dillera. Hilt Muc. T. I8. f. I7. A.

Habitat ad muros \& truncos arborum.

185. Lichen lemigerus - crultaceus orbicularis lo. batus flexuolus fubmbricatus albus; fcutellis demum convexiulculis flavorufelcentibus, margine albo.

Placodium lentigerum. Svet. Lintslaf.

Lichen lentigerus. Wéberi. Swartz. Act. Upf. T. IV. Reez, Pr. 2. Liljebl. Dicks. Hoffm. Enum. Lich, Schrad. Spicil, Villars, Relh. Wither. Bellardi.

Lichen lentiger. Roth. Tent. Gmel. Sylt. Linn. Pfosa lentigera Hoffm. Pl. Lich. \& Deut. Fl. Icon. Weber Spicil. H. Germ. T. 3. Hoffm. Pl. Lich. T. 48. f. 1. Ejusd. Enum. Lich. T. 9. f. 4. Relhon. Cantabe. p. 430. tab. Habitat in locis montolis ad terram. 
186. Lichen faxicola - cruftaceus orbicularis 10. batus fubimbricatus pallido virefeens; icutellis demum convexis lutefcentibus, margine pallido crenulato.

Placodium faxicola Svet. Quartslaf.

Lichen faxicola Pollech. P1. Palat. Retz. Pr. 2. Roth. Tent, Gmel. Syft. Linn.

Lichen muralis Schreb. Spicil. var. a faxicola Liljebl. Retz, Pr. 2. Dickse Scholl. Roth. Tent. Wild. Prodr Wither. Hoffm. Enum. Lich, Gmel. Sylt Linn.

Lichen ochroleucus $W$ ulf. ap. Jacqu schrank? Fl. Bav.

Lichen luteolus Gmel. Sylt Iinn.

Pfora tnuralis Hoffm. Pl. Lich.

Lobaria muralis Hoffm. Deut. Fl.

Icon. Hoffm, Pl. Lich. T. I6. f. I. Ejusd. Enum. Lich, T. II f. I. Jacqu. Coll. 2. T. I3. f. 4. a. Muchcl. Gen. T. Sr. f. 4. Habitat fupra faxa \& ad parietes ligneos vetuftos.

187. Lichen demisfus - cruftaceus cinereo-virefcens granulato plicatus, ambitu fubimbricato; lacinulato fcutellis convexiufculis rufo-fufcis, Placodium demisfum. Svet. Lâglaf.

Lichen demisfus Rutfróm. Disfert. Habitat in uda humo primo vere. Obf. Crufta irregularis effufa ad ambitum fublo. bata, laciniis minutis crenatis parum elevatis fubimbricatis, fubtus atris. Scutella immarginate adpres/e. De loco inte". Placodia of PSoromas ambigit hic Lichen.

I88. LrCHEN impresfus - cruftaceus luborbicularis verrucofus albido cinerafcens, ambitu folia. ceo lobato; fcutellis concavis immerlis glaucoceliis, 
Placodium imprestum. Svet. Hélklaf. Lichon imprestus Swartz. Nov, Act. Upf.4* Liljebl. Retz? Pr.

Lichen mulcorum Scop. Sehrod. Spicil.

Lichen bryophilus Ehrh. Cr. exl.

Lichen Lcrupolus var. B. Bofm. Enum. Lich.

Pora mulcorna Hoffm. Detr. Wl.

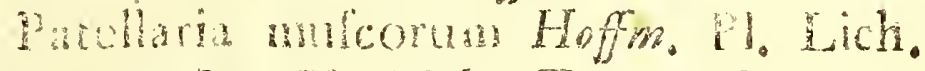

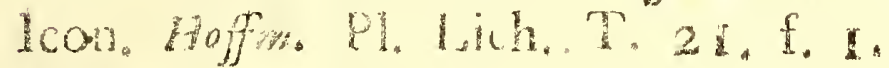

Habiat art mes in terra lupra mencos deftructos.

Obf. Forma os udole foutellarum Urceolarias ne-

fris propengens, nec holitus onnino Placodii;

- fed ab ambirus crufe foliaceum non poteft non Placodics asfofari of hac nota ciram a L. ferupolo difingui.

t-I. abefoens - cruitaceus fuborbicularis albicans corrugato lobatus; foutclis pallidis. - Plo. ra albefcens Hoffor, Deut. Fl.

Piacodium albejcons. mur. laxa. rup.

+ L. epigens - crultacens orbicularis albidus ambiul lobato; foutellis demum convexis nigris, margine albo. - Liehen epigeus Per/oon JUt. - Annal. d. Bot. St. 7. - Lichen melanocar. pus Ejusd. ibd. p. Is 5. - Lichen candicans Dicks. Cr. Br. 3. T. 9. f. 5.-Plora epi. geja Hoffm. Deut. F!.

Placodium epigenm. rup' terra, inter mulcos.

+I, fympageus - cruftaceus orbicularis fubimbrica. tus obfcure aurantiacus, a mbitu lobato foliaceo; fcutellis minutis concoloribus. - Lichen aurantius Perfoon. Ult. Ann. St. I I. Obf. An. L. elegantis varietas? Placodium fympageum. lap. calcar. G 5 
* J. cocoès - crufaceus orbicularis candidus fub. imbricatus adpuesus finuato laciniatus; fou. tells thatis atris. - Lichen cocoës Swarto. Irodr loon. ined.

Obt. Habius omnino Imbricaria. placodium coccës, in Tunc, Coc, nucif. Jamaic,

*. piens - cruftaceus obicularis allidus fubimbricaus fmatolo-batus multifidus; (cutellis atris margine albo _- Lichen pictus frwartz. Jrodr, lcon, ined.

Oh. An. varietas pracedentis? placodizum pilum. cort. arb.

t I. vorisiblis - crufaceus dilute umbrinus, ambi. tu allitio cinereus fublobatus; fcutellis con. feris fufcoczilis, margine albo - Lichen variglitis perfoom. Uft. Annal. d. Fot. S.7. - Hora variabilis Hoffm. Deut. Fl. Plocodinom variabile. lap. calcar.

†I. verferolor - cruffaceus orbicularis fubimbricauns cinereo alboque virefcens, ambitu muls if no toblobato; foutellis parvis confertis planiviculis fubmfis, - Lichen verficolor Perform. Uft. Am. St. 7. - Lobaria verficolor Ifeffm. Ievt. Fl.

placodium verficolor. lap. calcar.

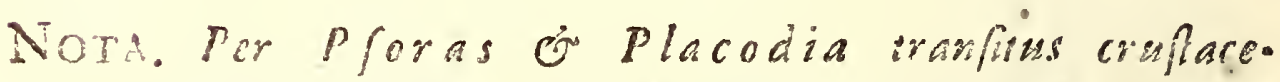
orum Lichenum ad foliaces optime demonfratur er fec intima propinguitate connedurtur Lichenes facile omoss. Lichen cxinus, incurvus, $E$ n. docarpa mea atque fpecies alia foliactis quiacens - adnameranda, fed naturam itidem produnt cruffes. ceis proximam. 


\section{LICHENES FOLIACE.}

\section{Tribus r.}

IMbricaria Svet. Táckapvar,

Folia membinacea (raritis fubecufacea fracillora) plano depresfa imbricatim centrifug?, rofulam formantia, la ciniato incira atque pinusta, lobata; fubtus fibrillora.

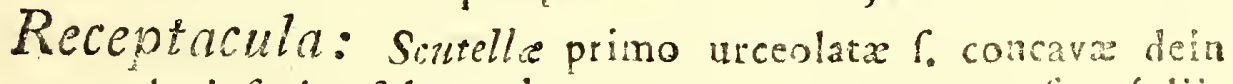
planiufcula fubmembranacex, centro tantum fino folis affixa, circumcirca libera, elevate, maxinatx.

Glomeruli in quilusdam lparii, centrales \& marginales puiverulenti.

* I. Foliorum laciniis angugioribus os linearibus.

189. Lichen insurvas - crufac o-membranacons fubimbricatus cinereo viridis, follolis nulti. fidis angutatis convexis mareine apicegie incurvis; glomerulis concoloribus; fcutulis rufis.

Imbricaria incurva, Svet. Kramafaf.

Lichen incurvas perfoo\%。 Ut. Ann. A. Bot. St 7 . Iichen multiglus Dicks. Cr, Br. Rufr. Dise, Lobaria incurva Horfom. Deat. W1.

Icon. Dicklon. Crype. Brit. 3. T. 9. f. \% Habitat ad montes.

Obf, Scutelle concava margine pallido.

I90. Lichan carius - cruftacen - mambanacens fubimbricatus cinereo alhidus, follohis laciniaro multifdis; glomerulis fparts pulverulen. tis calis; fcutellis nigris margine albicante. 
Imbricaria cafia. Svet. Blägrâlaf.

Lichen cæitus Hoffm. Ln. Lich. Ruter. Schrad. Spicil. Gmol. Byll. Lim.

Licher pulvernenus Weftr. Ad. Sc. Svec,

179\%. exclut. fynonym.

Lichen plora Dicks. Cr. Britt. 3 .

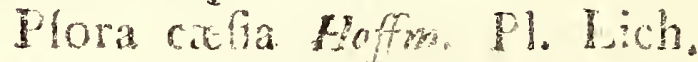

Lobaria cxilu Hoffm. Deut. Fl.

Icon. Hoffm. P1. Lich. T. 8. f. I. Eiusd. E-

sum Lich. T. 12. f, 1. Jacqu. Coll. 2. T. 16 f. 2.

Habitat in faxis, fupra mufcoso putrefactos, cortices \& ligna demortua arborm.

192. Licues leptaleus - cruftaceo-membranaceus jmbricatus cinereo-cæulus, foliolis lineari mul. tifilis angultatis flexuofis fibrillofo fubciliatis; fcutell:s fufco nigris margine albicante. Imbricaria lepalea. Svet. Kratlaf. Habitat ad faxa cum priori.

Obi. Glomeruli parvi marginales of terminales. $\mathrm{C} i$ -

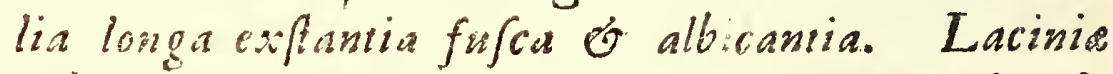
fubiess fere concolores viot canaliculata fufco fobrillofe.

Imbricarias es Phy feias conneltens medium quafi locum tenet inter I. crefium, ftellasem of tenellum; ab his vero omnibus, ut videtur, fasis diffridius. Forfan huc referendus: Lichen temipinnatus Leers herb. Gmel. Syje. Linn. \&

Lobaria femipinnata Hoffm. Dest. Fl. Jubims. bricatu degresfa mulufida ciliata Jubtus canalicatlata, feutellis wrgricanibus. Dillen. Hift. Mufc. T. 20.f. 46. A B. Wulf. ap. Jacqu。 Coil. 4. T. 6. A. b. C.

392. Lichen virellus - cruftaceo membranaceus fubimbricatus cinereo viridelcens, foliolis lobatis 
batis obtufis; glomerulis fparis pulverulen. tis viridibus.; fcutellis rufis marginatis.

Imbricaria virclla. Svet, Grägrónlaf.

Habrat in faxis.

Obr. Virtdisimus fit dum humeitatur, quod non valet de L. cæcio. In laciniis foliorum granula cuccinea Jparla in recenti Lichene evidentiora. Murgines forteilarum foliis concolores.

393. LICHEN agrilus - cartilagineo-membranaceus imbricatus fufco nigricans fubtus pallidus, fohois montatis angultatis convexis margine incurvis; fcutells nigricantibus. Irobricarat aguala, Svet. Flätlaf.

Lichov fulcus Hudf. Rutfir. Hoffm. En, Lich, wher.

I then prillus Lightf. Fl. Scot.

Lichen oblcurns Gmel. Sylt, Linn.

Icon, Dillen. Hilt. Mufc, T. 24, f. 69. Michel. Gen. T. 5I.f. 6?

Habitat in faxis \& rupibus.

Obf. Variat laciniis latioribus sore tertibus, orhucularis $\dot{\mathcal{O}}$ fubcaspitofus, laciniis implicatis tenerrimis, etiam colore hinc inde pallide fujco o albo cinerafcente, fubtusque nigracante. $F_{i}$ brille atre paginam inferiorem Jape obtegunt. Scu. tellie crenulate.

194. LICHen Aygius - cartilagineo-membranaceus imbricatus utrinque nigro piceus, foliolis multifido fubpalmat is anguftatis convexis margi. ne incurvis; fcutellis concoloribus margine crenulato.

Imbricaria figia. Svet. Bläkullslaf.

Lichen flygius Limn. Retz. Prodr. 2. Liljebl. Haffb. Gunn, Dicks. Hoffm. Enum, Schrad. Spicil, Mohr. Gmel. Syft, Linn. 
Squamaria Atygia Hoffm. Pl. Lich. Lobaria liygia Hoffm. Deut. Fl. Icon. Hoffm. P]. Lich. T. 2 5. f. 2. Eims. E. num. Lich. T. I4. fo. 2.

Habitat in faxis.

195. LICHEN fahlunenis - membranaceus imbrica. tus utrinque niger, foliolis multifido dichotomis angutatis planiufculis acutis margine fubreflexis crenulatis papillofis ; Icutellis fufcis. Imbricaria fahlunenfis. Svet. Fahlulaf.

Lichen fablunenfs Linn, Rerz. Pr. 2. Hoffl. Liljebl. Fuds. Light, Web. Gacqu, Neck. Meth. Craniz. Hofm. Enum. Roth. Tent. Bellardi. Wither, Mohr. Gmel. Sylt. Linn. Squamari. fahlunenfis Hoffm: PI. Lich. Lobaria fahlunenfis Hofm. Deut. Fl. Icon. Dillen. Hilt, Muc. T. 24. f. 8I. 7 acges. Mifc. 2. T. 10. f. 2. Hoffm. Pl. Lich. T. 36. f. 2: Ejusd. Enum. Lich. T. 1\%. f. 2. Habitat in rupibus \& faxis.

Obf. Differt a L. ftygio or affinibus: foliit, ob

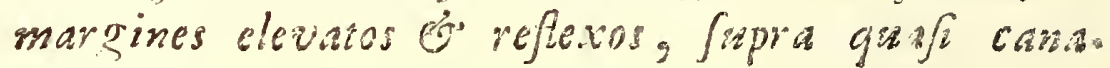
liculatis.

196. Lichein hepatizon. - membranaceus imbricalus caftaneus fubtus niger, foliolis multifidis tub. canaliculatis; fcutellis margin granulofis demum convexis flexunfis xneofufris.

Imbricaria hepatizon. Svet. Lefverlaf.

$H$ hitat in montibus elatis.

ObC. L. fahlunenfi proximus a quo pracipue differt colore, of futellarum forma, in primis quan. do adolefcint. Lacinia folionum ad centrum consiatc quemadmodum in I, olivaceo, ad ambitum vero multifide of ejusdem fere frublure ac in I. fahlunenfi, marginibus foilicet Jubreflexis atque interdum granulato papillofos scutelle junio. 
juniores concave concolores, margine gramularo coronate; per asatem magne fexuofe inaguales convexe colore magis daluto aneo-fufcefente, margine incarvo, \& hac vol plane deficiente vel rorius anturs crenulato.

197. I InEN fellaris - membranaceus imbricatus uborbiculai is cinereus, tubrus albidus cinereo fibrilons; foliolis multitios, laciniis convem ris; fcuthlis callo nigicantibus.

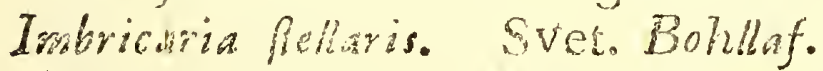

Lichen fiellaris Limn. Retz. Pr. 2. Liljebl. Hoffb. Scop. Buds. Lightfo Allion. Gmel. Tubing. Reich, El. Weis, Mull. Neck. Schreb. Spicil. Lecrs. Pollich. Matmer. Scholl. Wigg. Hogen. Leys. Reys. Hoffm. Enum, Wald. Prodr, Roth. Tent. Mohr. Var, a With. Lumsizz, Ginel. Sylt, Linn.

Toharia hellaris Hofm. Dent. Fl. Icon. Hoffo, Enmm, Lich, I. I3. f. T. 2. Dil: len. Hit. Mufc. T. 24. f. 70. Michel. Gen. T. 13. F. 2 .

Habiat ar corticem arborum.

Obf. Folue cincreo albidis haud raro apice latio ribus, fcuelligue non nanquars fufcelcentibus aliquantum differt:

Lichen ambigus Ehrh, Crypt, exficc. Fed Specie vix difinitus.

199. I.ICEEN axhelinus - monbranaceus imbricatus albus, fubus nigro tomentofus; foliolis mulingis adprestis planis; foutellis confertis livinofulcis demum cafis 'nigrisque. Imbricaria ambelina. Svet. Bricklaf. Habitat in arborum cortice.

Obl. Omnium fere partium colore of fructura di. verfa, quin immo to:0 habitu a L. fiellari dif- 


\section{- I12. -}

fert; nec cum L. aipolio facile confundi poteft -o folio linearia, plana, alba, of foutellas aluter coloratas. Humedtatis colorem fervat, Scutlle frequentiones quars is confinibiss of foliorum la. cinis approsimote jubconcreta. Hujus loci pro. cul dubso eft:

Lichen ftellariformis Hoffm. Enum. I.ich. qui Lobaria \{tellariformis Ejusd. Deut. Fl, foliolis ablongis laciniatis, foutellis fufois.

199. Lichen aipolius - membranacens imbricatus cinereo crilus, fubtus nigrolibrillofus, folio. lis plariuculis incilo lobatis lobulis lateralibus inambentibus apice rotundatis; foutel. lis cxilis.

Imbricaria aipolia Sret. Barklaf.

Lichen aipolius Ehrh. Crypt. ex?.

Lobaria aipolia Eoffm. Deat. Fl.

Habitat in cortice antiqua truncorum Fraxini \&zc. ObP. Deffert a L. ftellari in eo, quod inegualiter plersmare crefcat, guod lacinice foliorus latiores, sminas convexe, ad latero fere incurribentes, fub.

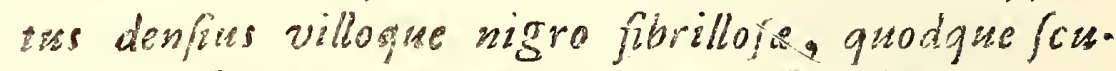
telia confranter colia mencant. - Madifaitus cosorems forvat; qua moia etiam a L. pulverulento werte difingratur. - Olim hic \& L. anthelirus cum l, itellari perperam commixtus fuit.

200. LiCHen pulverulengs - nembranacens imbricatus viridifufcus \%inern pruinatus fubtus nigro tomentofis; foliolis multifidis planis obtulis; forellis cxtiis.

Imbricaria pulveralesta. Svet. Stoftlaf.

Lichen pulverulentus Schreb. Spicil. Retz. l'r. 2. Wild. Prodr, Scoll. Wigg. Prim. Lumnitz. Scop? Hag? Web? Gmel. Sylt, Linn. Lichen anguftatus Hoffm. Enum. Lich.: 
Lichen fellaris var, b.'

Lichen orbicularis Neck. Delic, \& Metb.

Lichen allochrous Ehrh. Crypt. exf.

Lichen omphaindes Wulf, ap. Jacqu. Coll. 2.

Sumaria pulverulenta Hoffm. HI 'Lich.

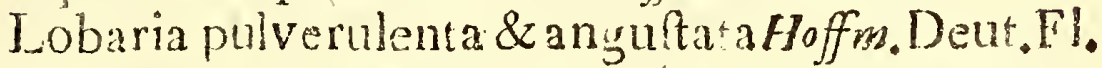
Icon. Hoffriz. P1, Lich. T. 8. f, 2. Ejusd. Enum. Lich. T, It. f, 2. 7acqu. Coll. 2. T.

15. f. 2. Dillen. Hit. Mufc. T. 24. f. 7 r. Habitat in cortice Populi trem. Tilix, Salicis. Obf. Variat laciniis latioribus of angufioribus. Deterfa farina, or per atatem, olivaceo fulcus evadit foutellis niovis. In utroque hoc fiatu expris mitur apud Hagen. Hif. Lich. 'T. 2. f. s. Madefactus late virefcii. Scutellarum margines per atatem crenulati.

201. LiCuen cyclofelis - membranaceus imbricatus cinereofucus, foliolis lobato multifdis an. guftatis margine granulatis pulverulentis; $\mathrm{fcu}$. tellis nigris margine cincrafcente. Imbricaria cyclofelis. Svet. Spridlaf.

Lichen diffulus, Wefring Act. Sc, Svec. 1791. Retz. Pr. 2.

Lichen orbiculiris Hoffm. Enum, Lich. Schrad. Spicil. Gmel. Sytt. Lion.

Lobaria orbicularis Hoffm. Deut. Fl. Icon. Hoffm. Enum, Lich, T. 9. f, I. Habitat in faxis \&z cortice arborum.

Obf. Folia in hoc fere femper inabricata, at non item in fequente, nec foutella unguam fubius ciliate. Glomeruli foliis concolores mediam $\mathrm{Li}$ chenis partem interdum occupant.

202. LicHen ulothrix - membranaceus fubimbricacatus fufco glaucefeens, foliis lineari multifidis planiufculis fcutellisque fubtus ciliatis nigris, margine albo. $\mathrm{H}$

Im. 
Imbricaria siloshrix, Svet. Borflaf.

Lichen ciliatus Hoffm. Enum. Lumnitz.

Lichen oblcurus Ehrh. Crypt. exl.

Lobaria ciliata Hoffm. Deut. Fl. Icon. Hoffm. Enum. Lich. T. 14. f, I. Dillen.

Hiti, Mulc. T. 24. fo 72.

Habitat in truncis Populi tremula.

Obf. Variat foliolis angufioribus of latioribus, cinereis of fufco nigricantibus, margine integris \& verrucoso pulverulentis. Cilia foutellarum per atatem evanefount of ipfa foutelle margine flexuofe irregulares crenulata objervantur. A pracedente difinitum ex habin es colore convincimur.

203. LiCHCN omphalodes - membranaceus inbricatus grifeo atuginolus glaber nigro punctatus fubtus niger; foliolis multifidis anguftatis linearibus planis truncatis; fcutellis fufcis. Imbricaria omphalodes. Svet. Letlaf.

Lichen omphalodes Linn! ( Retz.Pr. 2. Hoffl. Lilgebl. Hudf. Pollach. Lighefo Reich. Fl. Crantz. Hag. Neck. Leers. Wigg. Roih. Tent. Mattule. Mull. Mohr. Wither. Gmel. Sylt. Kinn. )?

Icon, Dillen, Hift, Mulc. T. 24, Michel, Gen。 T. 49. f. 2. mala.

Habitat in laxis \& lapidibus.

Ob!. Fariat colore incano, glanco, cinereo nigrio cante, fufco nigricante $\dot{F}$ omnino nigro, nec nor forma of difpoftione foliorum fingulari, unde too tus Lichen haud raro pannum fquamofuin quodammodo refert. Raro orbicularis.

Species hac difinctisfima, fi poft Linnaum non plane negletia camen a paucis fortasfis nota vel cum alis o pracipue cum L. faxatili injuRe confufas, unde etiam fynonyma inceria. Extra borialem nofiram plagam forte non crefcat. 
204. LiCHen faxatilis - membranaceus imbricatus cinereoglaucus fcabriufculus lacunofo reticulatus fubtus niger, foliolis linuato laciniatis planis fubtruncatis; fcutellis badiis.

Imbricaria faxattlis. Svet. Fárglaf.

Lichen faxatilis Linn. Thunb. Jap. Retz. Pr. 2. Liljebl. Hoffb. Gunn. Huds. Alloni. Lightf. Wigg. Pollich, Leers. Mattusc. Leysf. Mullo Hoffm. Enum. Mohr. Wild. Prodr. Wulf. Schrift. Nat. fr. Neck. Scop. LaMarck. Houtt. Ralh. Tent. Murray Prodr. Relhan. Lumnitz. Wither. Gmel. Syft, Linn.

Lichen laciniatus var, o \& $\beta$ Weis. Hag. Reich. Fl.

Lobaria Caxatilis Hoffm. Deut. Fl. Lobaria omphalodes? Hoffm. ibd.

Icon. Hoffm. Enum. Lich. T. 15. f. I. T. I6. f. I. Jacqu. Coll. 4. T. 20. f. 2. Dillen. Hift. Mufc. T. 24. f. 83. sichel. Gen. T, 49. f. I. Moris. Hift. T. 7. f. 6.

Habitat in faxis, fxpibus \& antiquis arborum truncis.

Obf. Variat laciniis profunde incifis angupioribus \& finuatis latioribus rotundatis. Vetufum jam Lichenem repralentat:

Lobaria adufta Hoffm. Deut. Fl. Scabra granulofa cinereo nigra, foutellis concoloribus.

Interdum folia etram maculis nigris adfperfa funt. "Propinguus I,. omphalodis, fed foliv. latioribus asperis of lacunofo reticulatis bene diftinctus. .

205. LICHEN phyfodes - membranaceus fubimbricatus glaber albo glaucefcens fubtus nigro fufcus, foliolis multifidis convexis apice obtufist. fub. inflatis; Icutellis rubris.

Imbricaria phyfodes. Svet. Queslaf. 
Lichen phyfodes Linn. Thunb. Tap. Retz. Pr. 2. Ziljebl. Hoffb. Pollich. Lightf. Reich. F\}. Matrifc. Wilf. Schrift. Nat. Fr. Ha. gen. Neck. Allion. Roth. Tent. Foffm. Enum. Gurn. Wigg. Schreb. Spicil. Schall. Leys/. Doerr. Leers. Mull. Relhan. Mohr. Lumnitz. Wither. Gmel. Sylt, Linn.

Lichen lacinianus var $y$ phylodes $W^{r} e i s$. var.

$\gamma$ tubulolus Hag. Hit.

Lobaria phyindes Hoffm. Deut. Fl.

Icon. Sonverby Eng. Bot. T. I 26. Hoffm. Enum. Iich. T. 15. f. 2. 7acqu. Coll 3, T. 8. Dillen. Hift Mufc. T. 20. f. 49. A D. Michel. Gen. T. 50. F. 1. 2, Pativ.Gaz. T. 14. f. 6. Buxb. Cent. T. I 4. fo I.

Habitat in cortice Betula albæ ad frpimenta lignea \& faxa inter mufcos.

Obf. Variat laciniis margine nigris of apice glo. merulato pulversulestis, nec non foliaceo expänfus rugofo plicatus es ad ambitums roundalo 10 . batss.

Singulari metamorphof, ut etiam in I. te. nello contingit, apices laciniarum matura fo pulvere prolificante? foere expanduniur.

206. Lichen diatrypus - membranaceus fubimbricatus depresfus cinereo pallidus fultus albus atro fibrillofus, foliolis mulifidis fupra foraminulofis apice fubinflatis; fcutellis minutis rubellis.

Imbricaria diatrypa. Svet. Stynglaf.

Lichen pertufus Schrad. Spicil, Schrank.

Lichen phyforles Wulf. ap. Jacqu. Iobaria terebrata Hoffm. Deut. Fl. Icon. Jacqu. Coll. 3 T. 8. f, I. Aniftra. Habitat ad truncos arborum. 
Obf. Folia hinc inde fupra poro notata of pertufa vix imbricata, fed plana os cortici adpres $\int a_{0}$. $A$ L. phyfode difincta ipecies, licet fimillima.

207. LrCHEN aleurites - membranaceis imbricatus rugofus albefcens farinofus fubtus cinerafcens fibrillolus, foliolis multifido lobatis crispis crenulatis; fcutellis elevatis ferrugineo fulcis, margine albo crenulato.

Imbricaria aleurites. Svet, Klilaf.

Lichen pallelicens Hoffm. Enum. (excl, fy. nom, \& fig.)

Lichen diffulus Dicks Cr. Br. Britt. (excl. fyn. Web.).

Lobaria diffufa Hoffm. Deut. Fl.

Icon, Hoffm. Enum. Lich, T, IO, f, 2. Dicks. Cr. Br. T. 9. f. 6 .

Habitat ad corticem Pini fylv. in trabibus \& palis antiquis.

Obf. In hoc pariter ac in Lo lanuginofo folia Sape evanefcunt of foutella fole remanent, farisa foliorum in glebulos congefia tantum conspicua.L. ambigui affinis,

208. LICHEN ambiguus - membranaceus imbricatus pallidovirefcens fubtus niger, foliolis nultifidis anguftatis linearibus planis; glomerulis concoloribus; Icutellis planis fufcis.

Imbricaria ambigua. Svet. Bjelklaf.

Lichen ambiguns $W$ ulf. ap. Jacqu. Coll, 4. Lichen diffufus $W e b$. Spicil. Fl, Goett. Lichen centrifugus var. B, Liljebl. Sv, Fl. Squamaria ambigua $\mathrm{Hoffm}$. Pl. Lich. Lobaria ambigua Heffm. Deut, Fl.

Icon. Jacqu. Coll. 4. T. 4. F. 2. Hoffm. PJ. Lich. T. 40. f. 2. -4 T. 42 . f. 2. 3.

Habitat ad asferes \& truncos femiputridos nec non in cortice Betulae albre.

Obf. 
Obf. Differt a L. centrifugo, cui proxime acce. dit, centro perennante of giomerulis pulveruleniis, laciniis omnibus linearibus, planis, fubtus nigris, foutellisque planis; a L. aleuritide $\&$ confperfo foliis linearibus. - Colore cenereo of glaucefcente interdum variat; occurrit etiam totus pulverulentus.

209. LiCHEN centsifugus - membranaceus imbrica. tus alboviref́cens fubtus albus, foliolis multifidis lineari lobatis obtulis; fcutellıs rufofulcis. Imbricaria centrifuga. Svet. Vinterlaf:

Lichen centrifugus Linnai.

Habitat ad faxa \& rupes.

Obf. Modo crefcendi per circulos concentricos remo. tos, (iguod hujus fingularis nota omnino eft) co. lore Suhtus albo (fibrille tamen nigre). (cutellis: gue verfus peripheriam inprimis locatis, nec centralibus ut in L. confperfo ab hoc diffinguitur, quamvis cum eo nullo jure olim confusus.

Synnnyma Auctorum, qui L. centrifugum nominarunt, utpote valde incerta, addere non po. tsi. Boreales incolit regiones hicce Lichen if an extra patriam noftram invenius fit, valde dubito.

210. Lichen con/perfus - membranaceus imbrica. tus glaucovirefcens nigro punctatus fubtus nigrofufcus, foliolis multifidis finuatis apice ro. tundato lobatis crenatisque; fcutellis badiis. Imbricaria confierfa. Svet Kaklak. Lichen confperfus Ehrh, in litt.

Lichen centrifugus Weis. Hagen, Hoffm, E. num. Lumnitz. var. A. Liljebl.

Squamaria centrifuga $\boldsymbol{H}$ offm. Deut. Fl. Lobaria contrifuga Hoffm. Deut. Fl. Icon, Hoffm. Enum, Lich. T. 10. f, 3. Ejusd. Pl. 
P1. Lich, T. 16 f. 2, Dillen. Hift, Mulc. T. 24. f. 75 .

Habitat in faxis \& lapidibus.

Obf. Variat foliis pallide virefeentibus pinnatiffdis, laciniis' longis, linearibus, varie complicatis, fubtus fufcis. H. I. Fig. 2. T. II. Fl Lapp: forte eft. Color, forma of modus crefcendi nec son analyfis difincium a priori ( L, centrifugo) affirmant. Aulioram jynonyma for fan non pauca, fi non fingula, ad L. centrifuguin olum relata, hunc Spectant.

* 2. Foliorum laciniis latiovibus rotundatis.

21I. Lichen fcoriens - coriaceo membranaceus ina. bricatus depresfus cinereo albidus nigro pulverulentus, fubtus ater hispidus, laciniis ro. tundato lobatis nitidis.

Imbricaria fcortea. Svet. Hudlaf.

Lichen tiliaceus Rutfroom Disf, Ehrh, fec. exempl. misfa.

Lichen lobatus Gmel. Syft. Linn.

Lichen Leers. herb. n. 955.

Habitat ad lapides \& arborum caudices.

Obf. Scutella ignota. $-A$ L. quercino f. tilia. ceo? Hoffm. (vide infra) cum quo forte com. mixtus, colore, punitis nigris Jupra fpariss o fubftantia fubcoriaced mulutum differt; jed L. confperfo magis affinis eff.

212. LiCHEN caperatus - coriaceo membranaceus imbricatus rugofus pallide flavefcens fubtus niger, foliolis finuato lobatis crenatis; fcue teilis rubris.

Imbricaria caperata. Svet. Getlaf.

Lichen caperatus Linn. Retz. Pr. 2. Liljebl.

Scop. Pollich. Allson, Huds, Neck. Weis. 
Eighif. Roy. Reich, Fl. Leers, Wulf. ap. Jacqu. \& Schrift. Nat. Fr. Lamarck, Guet. Gunn. Mull. Gorter. Reihan. Wild. Frodr. Wigg. Scholl. Reyg. Baumg. Schrank. Wither. Gmel. Sylt Linn.

Platisma caperatum Hoffon, Pl. Lich.

Lobaria caperata. Hoffm. Deut. Fl.

Icon. Hoffm, P1. Lich. T. 38. f. I. T, 39. f. T.

T. 42, f. I. Ejusd. Enum. Lich, T. 19. f.

- 2. T. 20. f. 2. Facqu. Coll. 4. T. 20 f. I. Dillen. Hift. Mulc. T. 25. 5. 96 Vaill. bot. T. 21. F. I2. Moris. T. 7. f. I. Michel, Gen. T. 48. f. r.

Habitat in faxis \& ad arborum truncos.

ODd. Haud raro pulverulentus, vix unquan orbicularss. L. confperfie of cortei propinguns. Habitus Lobaride.

213. Lichen plumbers - coriaceo membratiaceus inbricatus rotundato lobatus plumbeus fubtus cæruleo fpongiofus; fcutellis rubroferrugineis demum convexis fulcis.

Imbricaria plumbea. Svet. Blylaf.

Lichen plumbeus Lightf. Laljebl. Wither.

Lichen cærulefcens Hudf. Fl. Angl.

Lichen fquamofus Hoffm. Enum, Lich. Gmel. Syft. Linn.

Icon. Lighefoot. Fl. Scot. T. 26. Hoffm. Enum. Lich. T. 2 I. f. 2. Diller. Hift. Mufc. T. 24. f. 73. mala. Michel, Gen, T. 43. f. 1. mediocris.

Habitat ad caudices arborum,

214. Lichen lanugino/us - membranaceus tenerrimus imbricatus albo fubfulphureus farinofus fubtus nigro lanuginofus, foliis plicato rugofis rotundato lobatis; fcutellis minutis pallide fufcis. 
Imbricaria lannginofa. Svet. Hinnlaf.

Lichen lanuginolus Hoffin. Enum, Gmel. Syt. Linn.

Lichen membranaceus? Dicks, Cr. Br. Wrther. Icon, Hoffm, Enum, Lich, T. 10, I, 4. Dicks.

Crypt. Br. 2. T. 6.1.1.

Habitat ad rupes inque earum cryptis \& parietibus, fubftrata, ex mufis putrefactis, terra. Obl. Senefcens totus fit pralueve crasfo obdtuclus, foliis quibusdam vix confpecuis, fed horum reliquis tantrem fuperfitibibus, Scutelle rarisfinde,

215. Licuen parietinus - menbranaceus imbricatus fulvus fubtus pallidior, foliolis rotundato lobatis crispis; fcutellis concoloribus.

Imbricaria parietina. Svet. Vafglaf.

Lichen parietinus Linn. Thunb. Fap. Retz. Pr.2. Hoffb. Liljebl, Weis, Huds Ligh'f. Houg. Leers. Gstnn. Wild. Prodr. Matturce. Allion. Leys. Hoffm. Frum. Scop. Reich. Fl Humb. Scholl. Gmel. Tubing, Doem, Neck. Delic. Muli. Mohr. Wigs. Lumnitz. Schrank,Wither. Gmel. Sytt. Linn.

Lichen juniperinus? Neck. Meth. Lóbaria parietina Hoffin. Deut. Fi.

Icon, Sowerby Engl, Bot. T. 194. Hoffm. Enum. Lich. T. I 8. f. I. Dillen. Hift. Mulc. T.24. f.76. Habitat ad parietes, ligna, faxa.

Obl. Color per atatem fenfim abit in virefcentem \& ultimo fufco cinereum. Ip a folia tandem evanefcunt, foutelle copiofisfime funt \& totus Li. chen verrucofus ac pulverulentus evadit. Scu. tellas proliferas delin. Humb. Fl. Frib. T, 2, f, 2.

216. Lichen olivaceus - membranaceus imbricatus olivaceofulcus fubtus fufcefcens, foliolis ro. tundato lobatis nitidis rugolis punctatisque; fcutellis concoloribus,

Im. H 5 . 
Imbricaria olivacea. Svet, Olivelaf.

Lichen olivaceus Linn. Retz, Ir. 2. Liljeblo Hoffb. Huds. Wulf. Lightf. Weis. Reach. Fl. Schall. Reihan. Neck. Delic. Schreb. Spicil. Leers. Doerr. Pollich. Mattiifc. Hag. Leysf. Allion. Wigg. Hoffm. E. num. Wilden. Prodr. Lamarck. Hontt. Mull. Lumnitz. Mohr. Wither. Gouel. Syft. Linn. Lichen pullus Schreb. Spicil. Swarzz. Agt. Upf. IV. Retz. Fr. 2. Web. Hag. Allion. Neck. Delic, \& Meth, Wing. Wild. Pr, Roth。 Tent. Soholl. Gmel. Sytt. Linn. Lobaria olivacea Hoffm. Deut, Fl. Icon. Hoffm. Enum. Lich. T. 13. †. 3. 4. 5. Dillen. Hift. Mufc, T. 24, f. 77. $73, V$ aill. bot. T. 20, f. 8. Michel. Gen. T. SI. Habitat ad truncos arborum \& faxa.

Obl. Pariat foliis plus minusve rugo/ss \& crispis, nitides, opacis, punctatis, lividis of obfcuriorio bus; foutellarum margine integro (L. pullus A 4 . (lor.) \& crenato. Interdum totus fupra pulverulentus.

217. Lichen corrugatus - membranaceus imbrica. tus rugofus glaucovirefcens fubtus nigro fufcus, toliolis rotundato lobatis; fcutellis am. plis badiis, margine crenulato.

Imbricaria corrugaia. Svet. Paténlaf.

Lichen corrugatus Smith. Schrad. Spicil.

Iichen acetabulum Neck. Delic. \& Meth. Hoffm, Enum, Wulf, ap. Jacqu, Rutfr. Gmel。 Sy?. Lim.

Lichen patellatus Gmel. Syft. Linn.

Lichen ferratus Gmel. Sylt. Linn.

Lichen Leers, herb. n. 956.

Lobaria acetabulum Hoffm. Deut. Fl. Icon. Hoffm. Enum, Lich. T. I8. f. 2, Gacqu. Coll. 
Coll. 3. T. 9. f. I. Dillen. Hir. Murc, T. 24. f. 79. Vaill, Bot, T. 21, f. 13. Mich. Gen. T. 48 . f. 2 .

Habitat fupra corticem in truncis Aceris, Fraxini, Fagi.

Obl: Olim a quibusdam pro L. olivaceo! perpe. ram thabitus.

x.

* L. encanfus - membranaceus imbricatus albidus nicidus fubtus niger, foliolis lineari dichotomis; fcutellis ballis, - Lichen encauftus Smich, Act Soc. Linn. I. T. 4. f. 6. Obl. An fecundum Cl. Hofm. hujus lynony: ma funt? Squamaria pulla Bloffir. Pl. Lich. T. 32. f. 2, Ejusd. Lnbaria pulla Deut. Fl. \& Lichen multipunctus Ehrh. Cr, exf. Imbricaria encaufla. rup.

$\div \mathrm{L}_{0}$ dubivs - membranaceus imbricatus cinereoglancus fufco pulverulentus fubtus albus fuifcusque, foliolis multifidis lineari anguftatis con. vexiufculis; fcutellis fublufcis nigrisque. Lichen diffufus Hoffm. Enum. - Lobaria du. bia Ejosid. Deut. HI,

Obf. An L. cyclolelis varietas?

Imbricaria dubia. tegul。 fax.

t L. Speciofus - membranaceus imbricatus albo virens crerulelcens fubtus niveus, foliolis mul. tifidis lineari laciniatis ciliatis; fcutellis fufcoruis fubcrenulatis. - Lichen fpeciolus Wulf. ap. Jacqu. Coll. 3. p. 119. T. 7. Gmel. Syit. Linn. - Lobaria Ppeciola Hoffm. Deut. Fl. - Obl, Lacinix apice obtulæ glomeruliferæ.

Imbricaria Speciofa.

* L. 
* L, colpedes - membranacens imbricatus depresfus cinereo pallidus fubtus nigro fpongiofus, foliolis lineari mutitidis; fcutellis badiis. Obr. Habitus $I_{L}$, phylodis vel potius L. diatrypi. Imbricaria colpodes. cort. arb. Nov. Angl. Aner, susutz.

+ Lo, inteftiniformis … membranaceus imbricatus foli. olis multiclis lineari laciniatis elongatis compresfo teretibus articulatis depresfis; icutellis badius - Lichen intestiniformis Villars Delph. 3 Bellardi App. Pedem. Imbricaria irseftiniformis. lap. alpin.

t L. granofus - menbranaceus imbricatus lxvis ci. nereus, foliolis oblongis incilis lobatis; lcutellis nigris margine crenulato. - Lichen gra. nofus Schreb. Spicil. Hoffm. Enum. Reich. Wl. Scholl. Wid. Prodr.Roih. Tent. Gmel.Syft,Linn. Imbricaria granosa. ligu, putr. Salic.

f L. pityreus - membranacens fubimbricatus depres. fus, foliolis multindis margine crispis pulve: raceis apice lobatis obtulis crenulatis impres. fis pruinatis, - Lichen pulverulentus Ehrh. Cr exf - Lobaria pulveracea Hoffm. Deut, Fl. Imbricària pityrea. mulc.

$* 2$.

* L. guercinus - membranaceus fubimbricatus glau. cus glaber lub.us nigro hirtus laciniis obtufe lobaris; fcutellis branneis. - Lichen quercinus Wilden. 1. Berol. T. 7. f. 13. Roth. Tent. Grat. Sylt, Linn, - Lichen quercifolius Wulf, ap. Jacqu, Coll. 3. T. 9 f. 2. Gmel. Syit, Linn. - Lichen tiliaceus Dicks. Smun. Act. Soc, Linn, Hoffm? Enum, Lich. 
T. 16. f. 2? Gmel. Sylt, Linn, - Lobaria tiliacea Hoffm. Deut. F]. Michel, Gen, T, 45. f. I. - Cfr. L. Scorteus p. II 9.

Imbricaria quercina. cort. arb.

* Lo pamofus - membranaceus fubimbricatus ad. preslus cinereo fufcus venofo rugulofus, ambitu rotundo lobato, fubtus nigro tomento. fus; fcutellis convexis rufonigris. - Lichen pannolus Szvartz. Prodr. Icon. ined. Imbricára pannofá. Ind. Occid.

\section{Tribus 3 .}

\section{Collema suet. Klibbrafvar.}

Folia in ficco rigida dura cragiliora utplurimum nigra vel atro viridia; in humido gelatinofa, diaphana plus minusve late virefcentia \& tumentia; imbricata \& va: ga, lobata, fubtus fere nuda.

Receptacula: Scutella jam concava jam fubglobofx, urceolatx, planx \& convexx, fápius marginatx: lateraw les \& marginales.

* I. Foliis imbricatis minutis in recenti crasfes E turgidis.

218. Lichen granulatus - gelatinofus imbricatus oro biculatus, fóliolis lobatis crenulatis granulatis; fcuteilis urceolatis rutis, margine granulofis. Collema granulatum. Svet. Kornlaf. Lichen granulatlis Linn. Swartz. Act, Upf, IV. Retz. Pr. 2. Huds, Roih, Tent, Wither. Gmel. Sylt. Linn.

Lichen granofus Scop. Carn, Retz, Pr, 2? Schrank? Wulf? ap. Jacqu. Coll. 3. T, 10. f. 2 . Col- 
Collema granulata $W^{r} e b$. fuppl.

Collema granulolum Hoffm, Deut. Fl.

Icon. Dillen Hit. Mulc T. 19. f. 24. abs. que fcutellis. Fl, Dan. T. 462. f. I.?

Itabitat in locis arenolis apricis.

Obf. scurellis perfecte urceclais, quorum margines granulis glabcjes circumcirca coronain furt, opti. me oreliquis difinguarur.

219. Lichen crispus - gelatinofus imbricatus orbi. culatus lobatus crenulatus, foliolis centrali. bus crispis fubgranulatis; fcutellis planiufculis bailis fparlis.

Collema crispusas. Svet, Kronlaf.

Lichen crispus Linn. Retz Pr. 2. Liljebl. Huds. Lightf. Web. Pollich. Schreb. Spicil. Leers. Eilden. Prodr. Roth. Tent. Wither. Achar. Act. Sc. Svec. 1795. Gmel. Syit.Linu. Lichen murorum Neck. Meth. Collema crispum Hoffon. Deut. Fl. Collema crenulatum hoffm. ibd.

Icon. Dillen. Hift. Mulc. T. 19. f. 23.

Habitat in collibus liccis alibique inter mufcos. Obf. Scutella rarius immerfa of margines enrum non femper granulati.

220. Lichen limofus - gelatinofus imbricatus irregularis, foliols adpreslis minutis crenulatis granulofs; fcurellis fubimmertis rufofulvis.

Collema limofum. Svet. Dylaf.

Lichen granulatus Achar.ACt, Sc, Svec, I795. (excl. fynon.)

Iabitat in locis humidis, fosis argillofis, limo quali adglutinatus.

Obf. Loco crefcendi convenit, \& ex defcriptione Cl. Hoffmanni probabile fatis videtur hunc no. frum Lichenem eundem es/e ac Ejus: Collema graniforme Hoffm. Deut. Fl. 
221. LICren myriococcus - gelatinofus imbricatus ater, foliulis confertisfimis crispo granulatis; fcutellis aggregatis fubglobolis minutis concoloribus.

Collema myriococcum. Svet. Fingrynslaf.

Habitat ad rupes inter mulcos.

Obf. Scurelle fubglobofe minutus fime, ad margines of lasera foluclorum conferse, fupra paruori ix. cavate, difco, pundi tanium zmpresia forwans gerente, nudus oculis vix dafungmevido.

222. LICHEN crifratus - gelatinofus imbricatus glade co viridis, foliolis laciniato incifis oblolete dentatis; fcutellis e bafi foliorum magnis tu. Ifco rufefcentibus.

Collema criftatum. Svet. Kammlaf.

Lichen criftatus Limn, Lilicbl. Lightf. $N_{c} k_{0}$ Leers. Roth. Tent. Wither. Ach. Act, Sc. svec, 1795. Gmel. Sylt. Linn.

Collema crihatum? Hoffm. Deut. Fl.

Icon. Dillen. Hitt. Muic. T. 19. f. 26.

Habitat fupra terram in ficcis arenofis.

223. LICHEN marginalis - gelatinofus imbricatus, foliolis multifidis dentatis crenatis; fcutellis marginalibus nigrofufcis.

Collema marginale. Svet. Kantlaf.

Lichen marginalis Huds. Fl, Angl, Swartz; Act. Upl. IV. Retz. Prodr. 2. Wither。 Achar. Act. Sc. Svec. 1795 .

Lichen decumbers Oed. Eñum.

Lichen criftatus var. A marginalis Liljebl. SV. Fl.

Collema laciniatum Hoffm. Deut, Fl. Icon. Fl. Dan. T. 463. f. I. Dillon. Hift, Mufc. T. 19. f. 25.

Habitat ad terram, in rupibus \& laxis calcareis.

obf. 
Obf. Variat folits magis crispis \& angufioribus. - Differt a L. cioiltato fouteilis marginalibus folio minoribus nigrofufcis os fubfantia tenubri.

224. Liches frmatus - gelativofus imbricatus ferrugineo viridis pellacidus, foliolis laciniato inciris crispis depresfis; fontellis lpartis conco. loribus.

Collema finuatum. Svet. Fiklaf.

Lichen finuatus Huds. Fl, angl. Retz. Pr, 2. Liljebl. Relh. Wither? Achar. Act. Sc. Svec. 1795.

Colema finuatum Heffm. Deut. Fl. add.

Icon. Dillen. Hif Mulc, T. 19, . 33 . Bona. Habitat ad lapides lupia terram.

225. Lichen footinus - gelatinolus imbricatus atro. vire: $s$, foliolis minutis confertis creatufculis plicatogyrols lobatis crenulatis; futellis lateralibus concoloribus.

Collema fooinum. Svet, Suartbladslaf.

Habitat in locis campentibus graminofs terx

\& mufcis infidens

225. Lichen tenax - gelatinofus imbricatus depres. fus fubglancefcens, foliolis plano incumben. tibus lobato crenatis; fcutellis amplis fubim. merfis rufefcentibus.

Collema ienax. Sver. Limlaf.

Lichen tenax Swartz. Act. Upf IV. Retz. Pr. 2. Liljebl. Achar. Act. Sc, Svec, 1795. Gmel. Syst. Linn.

Collema lobatum, Hinfm, Deur. $\mathrm{Fl}_{\text {, }}$ Icon. Achar. Nov. Act. Ac, Sc. Svec. V. XVI. T. I. f. I. .

Habitat in ericetis montofis fupra mulcos, quibus admodum tenaciter adhæret. 
227. LiCHEN paitilis - gelatinofus imbricatus atrovirens, foliolis gyrolo-plicatis undulatis rotundato lobatis integrerimis; fcutellis Eeșilibus concoloribus.

Collema plicatile. Svet. Folllaf.

Lichen plicatilis Ach. 'Act, Sc, Svec. 1795. Icon, Achar. Nov, Act. Ac. Sc, Svec, $V^{\prime} \times \mathrm{VI}$,

T. 1. f. 2.

Habitat in faxis \& lapilibus quartzofis juxta aquam.

Obf. Folits in orben dispofutis integris ơ Joutellis jparfis Jesfilibus a L. faiciculari differt.

228. Lichen hydaterpers - gelatinofus imbricatus, atrovirens, foliolis plicato-undulatis incifo lobatis repandis creisulatisque proliferis; fcutellis parfis concoloribus

Collema hyduterpum. Svet, Vaitlaf.

Lichen fluviatilis Huds. Fl. Angl.

Icon. Dillen. Hitit. Mufc. T. 19, f. 28.

Habitat ad rupium latera aqua defluente irriga.

ta \& ad lapides calcarios litorales pasfim inundatos.

Obf. In aliis folia fat integra parfa difcreta, in aliis fubcafpitofa profitnde laciniata, palmato incifa of apice crenulata. - Siccus L. plicatili quodammodo fimilis, Jed folra profundius plerumque incifa atque lobata, margine obtufe crenulata, fubtus cinerascentia, fapius prolifera. Etiam in recenti durinfoulus or rigidus eft, fub. fragilis. - Defcriptio Dillenii bene quadrat, licet figura non optima.

229. Lichen fafcicularis - gelatinofus imbricatus, foliolis lobato crenatis plicatis; fcutellis marginalibus turbinato fubpedicellatis fafciculatis. J

Col. 
Collemsa fafciculare. Svet. Knipplaf.

Lichen fafcicularis Linn. Retz, Pr, 2. Liljebl. Huds. Web. Scop. Lightf. Ehrh. Cr. Exf. Relh. Roth. Tent. Wither. Ach. Act. Sc. Svec, 1795. Gmel. Syft, Linn.

Collema conglomeratum Hoffm. Deut. Fl. Icon. Fl. Dan。 T. 462, f. 2. Dillen, Hift, Mule. T. 19. f. 27.

Habitat in faxis \& montibus prefertim calcariis ad maris litora.

Obf. Scutella concolores per atatem fupra rufefcunt $\dot{\sigma}$ aliguando albefount, quod etiams rarius in L. plicatili accidit. - A noftro diftinctus omni. no videtzr $\mathrm{L}$. fafcicularis Wulf. \& Collema fafciculare Hoffm. (cfr. L. fymphoreus pag. 135).

230. LICHEN melanus - gelatinofus imbricatus ater, foliolis confertiscimis crispis undulatis incilis lacero crenulatis; fcutellis planis concoloribus margine granulato.

Coliema melanum. Svet. Strandiaf.

Habitat ad radices montium horizontales pro. pe ripas lacultres.

Obf. Orbiculation \& late aliquantum expanditur, atgue colore atro flation dignofcitur pulchra hacce fpecies, primo intuitu L. fahlunenfi fimillima. - Humidus obfcure virefcit. Scutslla in in fantia concave dersum cxplanantur of fat magne evadunt, margine frequenter granulis crenate rarius integro.

* 2. Foliis vix imbricatis latiorilus lobatis expanfis.

23I. Lichen nigrefcens - gelatinolus membranaceus virefcens, tolio dilatato fubrotundo lobato rugofo; fcutellis confertis rufis. 
Collema nigrefcens. Svet, Ldderlappslaf.

Lichen nigrefcens, Linn. Swartz. Aa. Upl. V. IV. Retz. Prodr. 2. Hisds. Ehrh. Leers. Roth. Tent. Wither. Reich, Fl. Mohr. Bellardi. Relh. Ach. Act. Sc. Svec. 1795. Gmel. Syit. Linn.

Lichen refpertilo Lightf. Liljebl.

Lichen papyracens $W$ ulf. ap. Jacqu.

Lichen lactuca $W c b$. Spicil.

Lichen crittatus Scop. Carn. 2.

Collema vefpertilio Hoffm. Pl. Lich. \& Deut. Fl. Icon. Fl. Dan. T. II25. f, 2. Hoffin. Pl. Lich. T. 37. f. 2. 3. Jacqu. Coll. 3. T. 10. f. 3. Dillen. Hift. Mufc. T. 19. f, 20.

Habitat ad caudices \& ramos Pop. trem, nec non ad rupes \& faxa.

232. Lichen faccidus - gelatinofus membranaceus complicatus atrovirens, foliolis rotundatis obtufe lobatis integriulculis laxis, feutellis fparfis concoloribus rufisque.

Collema flaccidum. Svet. Slinklaf.

Lichen flaccidus Ach. Act. Sc. Svec. 1795. Lichen rupeftris Linn. Syvartz AQ. Upl. V. IV. Retz. Pr. 2. Liljebl. Gmel. Sylt. Linn. Icon, Achar. Nov, Act. Ac, Scient, Svec. V. XVI. T. I. f. 4 .

Habitat in rupibus declivibus ttillicidio aqua. rum interdum madefactis.

Obf. L. deufto perfimilis quando faccus, itidemque haud raro pulverulentus, fed fubftantis gelatino. $\mathrm{Sa}$, foliorum \& foutellarum conformatione diffinctisfimus.

233. LICHEN rivularis - gelatinofus membranaceus glauco cinerafcens, foliolis oblongis obtufis fub. 
fublobatis integris laxis flexuofis; fcutellis pallide rubris.

Collema rivulare, Svet. Backlaf.

Habilat fupra faxa in rivalis.

Obl. Forma L. Flaccidi, at ab eo differt colore omnium partium alio.

234. LrCHeN furvus - gelatinofus membranaceus rugofo plicatus granulatus fufco virens incilus lobatus, lobis fubintegris undulatis; fcutellis Iparfis furcis.

Collema froroum. Sret. Rysklaf.

Habitat in cortice Pop. treimula inque faxis cal. cariis, adnatus.

235. LICHEN fatarninus - gelatinofus membranaceus atrovirens fubtus glaucus fubtomento. fus, foliolis fubrotundis integris undulatis; fcutellis elevatis rubris.

Collema faturninum. Svet. Samfklaf.

Lichen faturninus Dicks, Smith. Act. Soc, I.jun. I. Bellardi. Wither, Gmel, Sylt, Linn. Lichen difcolor Ach. Act. Sc. Svec. I795. Lichen myochrous Ehrh. Crypt. exl. Collema tomentofum Hoffm. Leut. Fl. Icou, Achar, Nov, ACt. Ac. Sc. Svec, V. XVI.

T. I f. 5 Dicks Cr. Britt. T. 6 f. 8. Habitat ad truncos Populi trem. Quercus, \& ad lapides.

236. LICHEN unaformis - gelatinofus membranace. us atrovirens, foliolis oblongis incifolobatis undulatocrispis; fcutellis fparis concoloribus. Collema tunaforme. svet. Vägbladslaf.

Lich: n turtormis Ach. Act. Sc, Svec, 1795. Icon, Achur, Nov, Ad.' Ac. Sc, Svec, V.XVI. 
T. I. f. 6. Dillen. Hitt. Mufc. T. 19. f. 29. Habitat ad latera prærupta montium calcariorum. Obf, $A$ L. opuntioide Wulf, omnino difinctus videtur \& Fig. Dillenii potius noftrum exprimit.

237. Lichen lacerus - gelatinofus membranaceus glaucefcens, foliolis confertis oblongis laciniatis margine laceris denticulato crispis; fcutellis minutis rubris.

Collema lacerum. Svet. Traslaf.

Lichen lacerus Szuartz. Act. Upf. V. IV.

Liljebl. Ach. Act. Sc. Svec. 1795.

Lichen granulatus var, $\beta$. Retz, Hr. 2. (excl. fynon.)

Lichen tremelloides Lightf. Weis, Neck. Web. Leers, Relhan. Wiiher.

Lichen crifpus Scop. Carn. 2.

Lichen tremella Roth. Tent. Gmel. Syft.'Linn. Tremella lichenoides Linn. Pollich. Hudf. Allioni, Collema finuatum Hoffon. Deut, Fl.

Icon. Dillen. Hift. Mufc. T. 19. f. 3 I.

Habitat inter \& fupra mufcos.

Obl. Infigniter variat sagnitudine of figara folio. rum. Inter varietases autem potisfimusom funt notande:

Collema ciliatum Hoffm, Dert. Fl. foliolis mara gine ciliatis. Dill. l. c. f. 31. A. B.

Collema fimbriatum Hoff ao. ibd. foliolis aggre. gatis fuccefcentibus, cilius dengis ramofos. Dill. l. c. C.

Collema pulviuatum Hoffmo ibd. foliolis lacini. atis truncatis confertusfruis putvinatus nigris. Dill. l. c. f. 34 . 


\section{$-134 .-$}

* I.

t L. bys fmas - gelatinofus imbricatus, foliolis mimatis granulatis; fcutellis demum convexis. - Collema byslinum Hoffen. Deut. Fl.

Collewa bysinum. terra limof.

†. Lo apareus - gelatinofus imbricatus nigrefcens, foliolis obliteratis; fcutellis depresfis rufelcentibus obfcure narginatis crenulatis. - Col. lema obfcurum Húfm. Deut, Fl.

Collessa aphancum. terra.

+ L. crispatellus - gelatinolus imbricatus, foliolis undulato crispis erectis in ambitu expanfis; fcutellis terminalibus. - Collema crispatum. Hoffm. Deut. Fl.

Collema crispaellims. rup. faxa.

I L. glyphicus - gelatinofus imbricatus finuato plicatus breviter multilobatus, lobis fuberectis rotundatis undulato crifpis una cum fcutellis tuberculofo crenulatis - Lichen crifpus $W u l f$. ap. Jacqu. Coll. 3. T. IO. f. I. - Obf. A L. crifpo, Linn. \& noltro omnino diverfus videtur.

Collema glyphicum. terra.

$\doteqdot$ L, cheileus - gelatinofus fubimbricatus diaphanus, fuliolis erétiufculis lobatis finuato crenulatis concavis; fctetlis crenulatis. - Collema marginale $H a j f m$. Deut. Fl.

Collema cheileum. mur, terra.

- + L. glaucefcens - gelatinofus fubimbricatus cinereoglaucus, foliolis crispo lobatis obtufis; fcutellis - amplis fufco fanguineis, - Collema glaucefcens Hoffin. 
Hoffm. Deut. Fl. - Lichen criftatus Wulf. ap.

Jacqu. Coll. 3. T. 12. f. I.

Collema glaucefcens. agger. mur.

f L. gyrofus - gelatinofus imbricatus, foliolis ver.

ticalibus margine fubxqualibus cxipitofis gy. rofo plicatis; fcutellis marginalibus lpadiceis. - Lichen gyrolus Gum. Kiz. Mrodr. 2. El Dan. T. 463. f. 2 .

Collema gyrofiam. terr.

f I. polycarpus - gelatinolus imbricatus aggregatus, foliolis lobatis rugolis, rugis elevatis fcutelli-

- feris, fcutellis confertislimis fubpedunculatis. - Collema polycarpon Hoffm. Deut. Fl. Collema palycarpuom. terr.

t I. Jymphoreus - gelatinofus imbricatus aggregatus, foliolis erectis gyrofo crifpis; fcutellis minutis obverfe conicis confertislimis.-Lichen fácicularis $W$ ulf. ap. Jacqu. Coll. 3. T. II. f. 2. - Collema falciculare Hoffm. Deut. Fl. a quo differt colore fordide luteficente: Collema argilloium Hoffm. ibd. - Obl, Species a L. fafciculari Linn. \& Noftro diftinctie. Collema fymphoreum, terr. fax.

+ L. Jynalizzs - gelatinofus imbricatus niger, foliolis crispis; fcutellis minutis obovatis conve$x$ is apice depresís in capitula fubftipitata connatis. - Collema botrytis Hoffers. Deut, Fl. Collema fynalizum. inter mufc.

* L. palmatus - gelatinofus imbricatus tenerrimus fubfulcus pelincidus, foliolis parmato incifis. - Dillen. Hit. Maluc, T. 19. f. 30. Scu. tellia 


\section{$-136 .-$}

tella ignotre. An h, 1. Lichen palmatus Hudf. Fl. Angl?

Collema palmatum. in apricis inter mufc.

* L. fubtilis - gelatinofus fubimbricatus fufcoviri.

dis, foliolis minutisimis angultis fubdivifis laciniis inæqualibus fubtruncatis; fottellis con. fertis demum plaris - Lichen fubtilis Schrad. Spicil. - Collema fubtile Hoffm. Deut. Fl. Collema fubtile. in viis, terr, limof.

t L. Sedifolius - gelatinofus imbricatus orbiculato convexus, foliolis teretibus fpongiofis virefcentibus fubus albidis; fcutellis lateralibus planis atris. - Lichen fedifolius Scop. Carn. 2. 395 .

Collema fedfolium. rup.

* 2 .

* Lo marginellus - gelatinofus membranaceus glau. covirefeens pellucidus, follolis lobatis undulatis; fcutellis minutis marginalibus ferrugineis margine. albo. - Lichen marginellus Swarti, Prodr. Icon, ined, - Dillen. Hilt. Mufc. T. 19, f. 32, - Collema marginale Hoffm. I'. Lich. T. 37. f. I.

Collema marginellum. inter mufc. Ind. Occ.

* L, tremelloides - gelatinofus membranaceus tener plumbeus, foliolis incifo lobatis, complicatis undulatis; fcutellis rubris lparfis margine pallido. - Lichen tremelloides Linn. Suppl. Gmel. Syit. Linn. - Lichen cochleatus Dicks. Cr. Br. I. T. 2. f. 9. - Collema plicatum Hoffm. Pl. Lich. T. 35. t. 2.

Collema tremelloides, in arb. inter mufc, Afr, A mer. t L. 
†. auricalatiss - gelatinolus meinbranaceus glau. cefcens, foliolis finuato lobatis crenulatis; fcutellis depresfis muricatis rafeicentibus, Collema auriculatum Hoffro Beut Fl. (excl. fyrom. Dicks.)

Collema auriculatums. arb. cort.

* Lo diaghanus - gelatiofus membranacens pellucidus tenermins caruleo virefcens, follolis plicato undulatis hacinis cripis; Icutelis parfis fermugess. - Lichen diaphanus Swarzo Prodr. Jcon, ined.

- Collema diaphinum. arb, Ind. Occid.

* L。 azureus - gelatinofus membranaceus tenerrimus carulelcens, foliolis rotundato lobatis undilatis integerrimis; (cutellis rubris margine albo. Collema azureum. Ind. Occ. Swartz. Icon. ined.

* L。 bullaizs - gelatinofus membranacens tener plum. beus pellucidus, foliolis obure lobatis undu. latis veficulofus; veficulis inferne apertis turbinatis fcotellfferis; fcutellis convexis rufis,Lichen veficulofus Swartz. Prodr. Ic, ined, Gmel. Sytt. Linn.

Collema butlatum, cort. arb, intermufc. Ind. Occ.

L. 3yrgeni -gelatinofus membranaceus atrovirens, foliolis rotundatis crenulatis crispis; fcutellis immerfis concoloribus margine elevato murica. ti crifpo. - Lichen Burgesfi Lightf. Fl Scot. T. 26. Linn.Retz Pr. 2, Hoffm. Enum. Lich。 T. 2 I. f. I. Gmel. Sylt, Linn. - Lichen ornatus Linn. Suppl. Murray \& Gmel Syft. Lin. Collema Burgesfii. trunc, Betulx, Coryli.

+ L. defcisfus - gelatinofus membranaceus imbricatus, foliolis lobatis laceris; fcutellis fufcis Is pro: 


\section{$\rightarrow 138 .-$}

proliferis, -... Lichen lacer, Gunn。Retz.Pr, I. Olafs Istant. Fl. Don. T. 470, f, 3.

Collewa deforsfarm. faxa.

t Lu pulcher - gelatinofus carnofus fubdiaphanus viridi-cerulefcens, foliolis timuato lobatis ex. afperatis, fructieris temioribus; fcutellis rufelcentibus margine crenulato tumido. - Li. chen pulcher Leers. Roth. Tent. Gmel, Syit. Linn. - Ijichen fubmarginalis? Wulf. ap. Jacqu. Coll. 3. - Collema prafinum Hoffm. Deat. F1. - Dillen. Hilt. Mufc. T. 19. f. 22. Collema patchrum. rup. terr.

† L. oprntinides - gelatinofus membranaceus proftra. tus, foliolis ramolo laciniatis difufis "ubarticulatis fubreniformi lobatis granulofis. - $\mathrm{Li}$ chen opuntioides Wulf. ap. Jacqu. Coll. $3 \circ$ p. 133. (cfr. Lo tunatormis, pag. 132.) Collema opuntioides. rup.

†. corniculatus - gelatinofus membranaceus livido fufcefcens, foliolis convoluto cucullatis multifidis comiculatis. - Collema corniculatum Hoffm. Deut, Fl. - Dilien. Hilt, Mulc, T'. I9. f. 30 ?

Collema cornicutatum. terr.

I. L. coriiformis - gelatinofus membranacens atrovirefens, foliolis finuato lobatis, lobis fuberctis repando crifpis. - Lichen coriiformis Wulf. ap. Jacqu. Coll, 3. p.142, Gmel. Sylt. Limn.

Collema coriiforme.

t L. jacobacfolisu - gelatinnfus membranaccus atrovirens, foliolis multipartitis crispis; fcutellis atro- 
atrofanguineis.--Lichenjacobærfolius Schrank. F1. Bav. 2. Gmal. Sylt. Limn, (excl. Syn. Wil len.) Vix differt: Lichen multifidus Scop. Carn. I. Reich. Fl. cír, Reich. Spec, Dl. Lim. p. 534

Collema jacobaafolizm. rup.

† I. fugax - gelatinofus nodofo plicatus fubrobatus hyolino nigricans. - Lichen fugax Wulf. ap. Jacqu. Coll. 3. T. 12. f. 2. Gmel. Sylt, Lim. An Tremella?

Collema fugar.

t. Is tenuisfmus - fubgelatinofus menbranacens im. bricatus fufcovirefcens, foliolis digicato anul. tildis, lacinis nubcilatis; foutellis fubmmet. fis fufco rufelcentibus margine obtufo. Lichen tenuisimus Dicks. Cr, Br. T. 2.f. Gmel. Sylt. Limn. - Collema teduistimm Hoffm. Dellt. FI.

Collema tenuisswam. agger, fabulof.

Nota, Lichen farcoides Whalfo ap. Facgis. Mije. 2. T. 22. Gmel. Sylti liono ad fungos migraver; mimiram: Peziza Hall. Elvela Schaff. Helvel. la furcoides Dicks.

Syronysa Andorsm in hac Tribu diffole expls cantur. Quanem in me fuie, tamen ander elaboravis

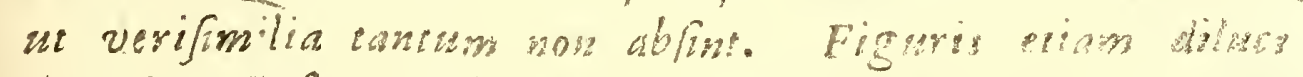

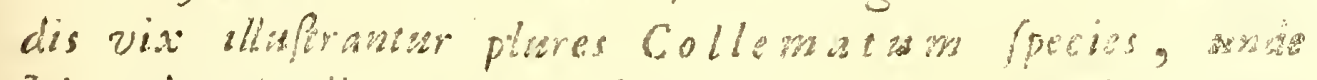

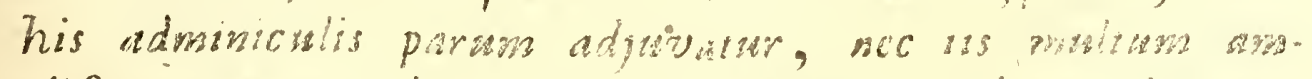

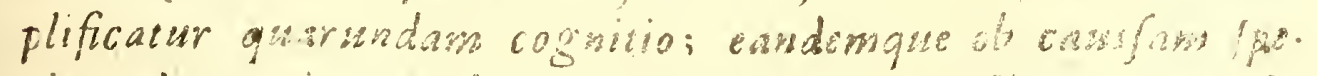

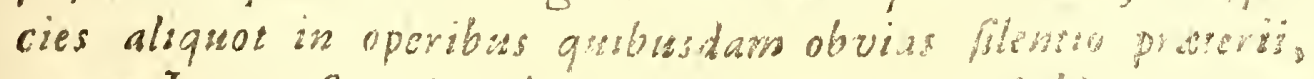

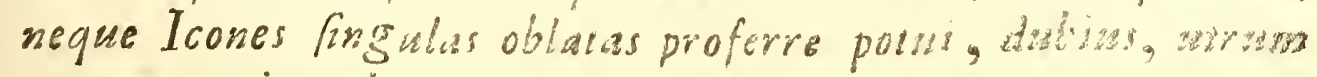

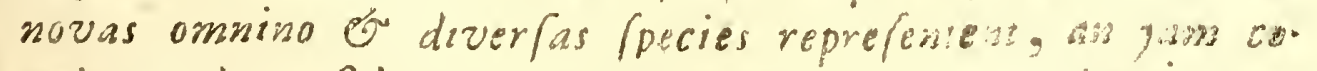

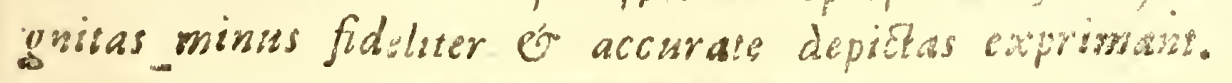




\section{$\therefore-140$. \\ Tribus 4 :}

Endocarton Svet. Lonskitafyár,

Folia cartilaginea dura rigida ceu fubcrufacea, roundata fubpeltata depresfa, pleramque difcreta vel etiam con. ferta \& vario modo flexuora, fribus nuda.

Receptacula: Thalani in ipra fubrantia folii nidulan. tes \& ablconditi, ad fuperficiem elevatam protuberantes, per $x$ tatem erumpentes \& oftiolo hiantes.

238. LICHeN Hadragii - fubcoriacens obfcure vim ridis fubus albus, foliolis difcretis minutis rotundato anguatis lobatisque; thalanis ni. gro fufcis.

Endocarpon Hedurgio Svet. Hedwioslaf.

Lichen trapeziformis Zoeqa. Retz, Pr. 2. Dicks. Olefsen Isl. 2. app. Wither.

Lichen endocaspon Gmel. Syt. Linn.

Endocarpon pufllum Hedvig. Crypt.

Icon. Heave, St. Cryte. T. 20. f. A. Dillen.

Hift. Mulf. T. 30. 1. 133. Michel. Gen, T. $54 . \mathrm{t} .3$.

Habitat fupra terram in rupibus, inter mufcos.

239. LICHEN lachneus - fubcoriaceus fufcovirefcens fubitus niger fponginfus; foliolis minut is fub. incumbentibus rotundatis flexuofo fubiobatis; thalamis nigris.

Endocarpon lachresm. Svet. Filtlaf.

Habitat in cryptis rupium fupra terram \& mu. fos putrefatios.

Obf. Folia plerumque ita conforta, ut ex parie fibimer Superincumbant, Juburs iomento den/o fpongiofo nigroforfco connexa 8 huic intime affixa, extremis tantum marginibies nudis, in ficco fubrugofis. 
240. LICnen cuplocus - fubcartilagineus ancovire: fcens fubpeltatus, fubtus pallide flavus, foJis mirutis aggregatis rotundatis flexuof fublobatis; thalamis nigris.

Endocarpum euplacuss. Svet. Vecklaf.

Habitat in faxis.

Obf. Bumethatus totus late virefcit \& elafficus fere eft, nec ita fragilis ut pracedentes. Subtus oculo armato fublacunofus.

24I. Lichen leprophyllus - fubcartilagineus peltatus nigrofulcus, fubus niger, folio minuto fublobato repando flexuofo; thalamis nigris. Eridocarpon leptophyllum. Suet. Lillbladslaf. Habitat ad faxa.

Obl. Statura o habitus L. miniati, An hujus varietas pygmaa? Per fe tamen crefcit, nec cum - co immixins, es color itidem diver fasfimus.

242. LICHen miniatus - fubcartilagineus peltatus aibocineralcens tenuistime excavato punctatus fithrus lavis demum rugofus fulvus, folio repando integro.

Endocarpon miniazum. Svet. Monjolaf. Lichen miniatus Limn. Retz. Pr. 2. Liljebl。 Gunn. Lighef. Allion Leysfo Scop. Reich. Roth. Tent. Mohr. Wizher. Mefri.A\&.Sc. Svec. 1793 . Gmel. Sylt. Linn.

Icon. Jargn, Whic, 2, T, 10 fo 3. Fl. Dan. T. $832 \%$ f. 1. Dillen. Hito Nuc, T. 300 f. 127. Hall. Hith. 3. T. 47. f. 2.

Habitat in faxis \&z rupibus.

Obf. Aliguando luxurat folits quibusdam e centro progerminantibus, negue tamen complicatis ut in Jequenti.

An punta illa nigricantia in hoc of Jeguen. tibus confpiona, vera fint Thalamorsm initia,

se. 
necimus; Aliquando enim in peciminibus ve:upioribus quedam horum majord, exflantio, modo Tricarum plicals of rugaha, videntar; tamen of

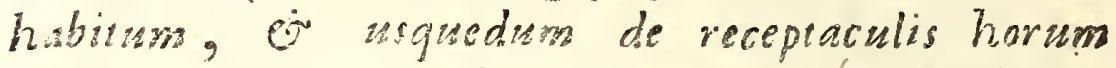
Leinensus certioses fieri posfmus, inter Endocarpa; quan Unbolicarias potius locardos es/e putavi.

243. Licren complicams - fubcartilagineus poly. phyllus inbricatus cinercolufcus punctis ele$V$ atis nigrofucis, fubtus fufco rigricans, foliobs erectideulis rotundatis plicato convolutis. Endocarpos compliatum. Svet. Vicklalaf.

Lichun complicatus Szortz. Ad. Upl. V.IV.

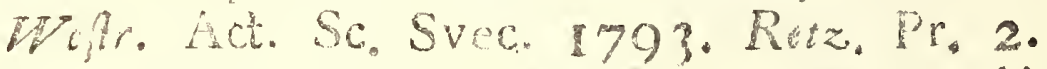
Lichen minatus var. B Ligh!f. var. A. Liljebl. Ico3. 7acqu. Coll. 2. T. 16. F. 2-i Fl. Dars.

T. 53 . 2. Machel Gen. T.54 A.I.Hall. liti. 3. T. 42. . 2 .

Habiat in moibus prefertim marnis.

Obl. Conformatzo folionsm fingularis a pracadestis

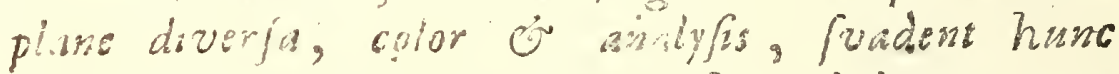
foulum proponere, quanvis L. miniato algese etivira L. Wiberi proxumus fit.

244. Licwes Webri _- Cubcanilagineus cinereo virens fitcr pinctatus funtus ochroleucus, folindis congen fasuohs irregulaibus lacinato lobsis.

Endocarpon Proboi. Suet. Weberslaf.

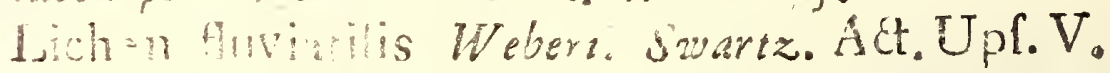
I. Ehrh, Roth. Tent. Gmel. Sylt. Linn. Itichen agtations Peir. Reich. Fl.

Iichen rehurnatus var, $B$, flaviatilis Liljebl. Hatisma aguatican Hoffm. Pl. Lich.

Lobaria aquatica Hoffm, Deut. Fl. Icon, Wr. El. Spicil. Germ, T. 4 Hoffm, Pl. Lich. $\mathrm{T}$. 
. T. 45. f. - 5. Dillen. Hif. Mufc. T. 300 f. 129. Nichel. Gen. T. 54. f. I?

Habitat in rivulis fupra lapides.

'Obf. Loco crefcendi es habizu diverfo a L, Com. plicato dipeneras, gnamus a Lighefootio cum co confundatur. Etiam Dillemiz ab eo citata fign. ra pouns hajus eft guan L. complicatio.

245. Lichen murinas - fubmembranacens, peltatus cinerens fubus nigro fulcus papillofo faber, folio plicato repando.

Endocarpon marintion. Sver. Blacklaf.

Lichen grifeus Swartz. Wir. Ac. Sc. Svec. 1793. Retz, Prodr. 2. Ach. Act, Sc, 'Sv. 1794.

Umbilicaria grifea. Hoffm. Deut. Fl.

Icon. Achar. Nov. Act. Ac. Sc, Srec, V. XV. T. 2. f. 3. Vaill. Paris, T, 2I, f, 14. (lec, Cel, Swith.)

Habitat ad laxa.

Obf. Species dafincissim etiamfi receptacula nondum reperta. - Inter Endocarpa iam retuli ob habi. tum, receptaculis vero detelis forte ad Umbilicarias transmigrabit. - Folume fubtus nigrum, ad ambitum tamen mogis fufcelcit, Juperficie in. feriori topa quanta pundtis elevaris winutis, ver. - Sus peripherzam Jubalbidis, adjperfa er Jubasperata.

* L. Thunbergii - cartilacgineo cruftaceus peltatus flavovirefcens, fubtus nigro fufcus, thalamis punctisque fparfis férugineo nigris. - Lichen viridis Linn. Suppl, Gmet. Sylt. Linn. Endocarpon Thunbergii. rup. Afr. Thune. Ic, ined. 
$\div$ L. retioulatus - fubmembranaceus peltatus multifidus niger excavato punctatus fubtus fufcus, lacinis radiantibus longitudinaliter conglutinatis, alis transverfalbus fectis, - Lichen re. ticulatus Olafsen Isl. 2 app. Retz. Pr,2.Gun. Endocarpon? reticulatum. rup.

\section{Tribus 15.}

UMBIIICARIA. Suet. SKÖLDLAFVAR.

Folia rigida cartilagineo membranacea, peltata, rotundara, depresfa integra laciniata; fabcus nuda aut fibrilloa. Receptacula: Trice fesfiles pianiufulx \& convexx immo globolx, rotundrax \& angulatx; plicis concentricis \& gyrofis, rugefis at glabris ornatx, femper atra.

246. IICned glaber - membranacens peliatus atrovirens fubtus ater utrinque nudus lrvisfimus, folio inzquali lobato plicato; tricis demum fubglobolis rugofis.

Umbilicaria glahra. Suet. Barlaf.

Lichen glaber Ach. Act. Sc. Svec. 1794.

Lichen pullus Weler. ibd. 1793.

Lichen anthracinus Walf. ap. Jacqua Dicks. Murray \& Gonel. SyR. Iinn.

Lichen polyphyllus Limo Retz. Pr. 2. Hoff'. Liljebl. Wefir. 1. c. Web. Lighef. Reich: Fl. Crantz. Neck. Meth. Gunn.Wigs. Roth. Tent, Mahr. Wither. Gmel, Sylt. Linn.

Umbilicaria polyphylla schrad, Spicil, Hoffm. Deut Fl.

Umbilicaria anthracina Hoffm. Deut, Fl. ${ }^{3}$ Icon. Achar. Nov, Aet, Ac. Sc, Svec, V, XV. T. 2 . 
T. 2. f. 5. 7acqu. Mifc. T. 9. f. 4. Dillen. Hitt. Muic. T. 30. f. 129.

ObC. I, arsut, vel potius luxuriat, foliis prolificis of mutetifariam implicatis, unde nomen polyphylli; Superne etians ater, fuliginofo glaucelcens of fuforereses, margine fubintegro vel erofo es crenulasu. - Nowan polyphylli non convenit, nam

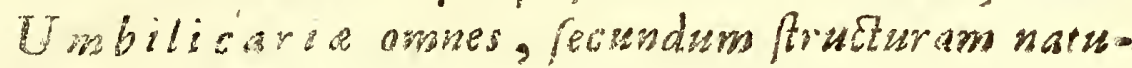

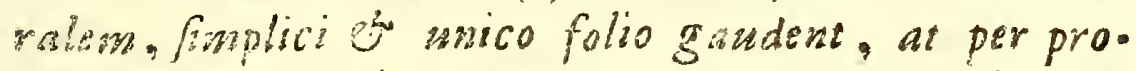
lificationem of luxuriom polyphylle aluguando eva. dunt.

247. LiCHen deup - membranaceus peltatus fufcus, fubrus impresfo punctatus lacunofus nulus lavis, folio fublobato; tricis planis dupplici circulo, denum convexis rugoils. Umbilicaria dorafta, Syet. Suedlaf.

Lichen deutus Linnei, Wefr. AR, Sc. Svec. 1793. Retz. Pr. 2. Liljeb?. Hejpo Gronsm (excl.ic. V aill.) Wither. Luwnitz, Schrank? Lichen flocculous Wulf. ap. Jacqu, Coll. 3 . T. I. f. 3? Gmel. Syft. Linn. Umbilicaria flocculofa Boffm. Deut, Fl? Habitat in rupibus apricis. Obl. Superficies anterior pulvere fuliginolo plerum. gue teita, inferior nus la concolor. - Varietas occurrit ambizus erofo fabcribrofo inftar $\mathrm{L}$, erofi, frobus atra profundiss lacunofa.

Pauci Austorum genuinum hunc Linnsi L. deutum rite intellexerunt, quod ex defcriptionibus eorum facile erisisur; fed frequenter cuon $L$. probolcideo Ejusd. confuderunt. Icon etiam defideratur.

248. LrCuen erofus - membranacens peltatus niger rugulofus cribrolus fubtus cineren fulcus libb. K. fibril. 


\section{- I. 6 6. -}

fibrillofus, folio erofo - laciniato; tricis pla. vis demum gyrofo plicatis.

Umbilicaria eroja. Svet. Siktlaf.

Lichen erofus Weberi. Suartz. Acte Up?. V. IV. Retz. Pr. 2, Roth. Tent, Ach, Act.

Sc. Svec, 1794. Gmol. Sylt, Linn.

Lichen corrolus Ehrh. Cr. exl,

Lichen reticularis Wefring. Act. Sc, Svec. 1793.

Lichen cribelium Retz. Prodr. F]. Sc, 2.

Umbillicaria torrefacta Sihrad. Spicil. (excl. fyn, \& fig.)

Umbilicaria erofa Hoffm. Deut. Fl.

Icon, Achar, Nov, Act, Ac, Sc, Svec, V. XV.

T. 2. f, 1 .

Habitat in rupibus prafertim mari propinquis.

249. LiCHEN hyperboreus - membranaceus peltatus fufco nigricans rugolo papulofus, fubtus lee vis obfolete lacunolus niger, folio erofo la. ciniato, tricis diformibus glabris nitidis. Umbilitaria hyporborea, Svet. Yfuerlaf.

Lichen lypenboreus Ach. Act.Sc. Svec. 1794. Lichen erofus Wefir. ibd. 1793. Liljebl.SV. FI. Umbilicaria hypesborea Hoffm. Deut. Fl. Icon. Achar. Nov, Ad, Ac. Sc, Svec, V.XV. T. 2. f. 2.

Habitat ad Japides in collibus \& montibus. Obr, Differe a L, deulto Juperficie anteriori papes: lofa - a L. erofo folio non cribrofo, colore or abjentia fibrillarum.

250. LICHEN pufulatus - membranaceus peltatus verrucofus papulofus cinereo virefcens fubtus lavis xruginofolufus profunde lacunofus, folio inæ. quali fublobato; tricis planis demum gyrolis. Umbilicaria puftrilata. Svet, Tujchlaf.

Lichen 
Lichen puftulatus Linn。 Retz. Pr. 2. Wepr. Act. Sc. Sv, 1793. Hoffb. Liljebl. Heds. Allion. Lightf. Wulf. Ley\%. Pollich. Mat. iuch.Web. Crantz. Neck. Roeh. Tent. Gort. Gunn. Leers. Scholl. Mohr. Wither. lum. nitz. Gmel. Sylt. Linn.

Umbilicaria puffulata Hoffm. Pl. Lich. \& Deut. Fi. Schrad. Saicil.

Icon, Hofm. Pl. Lich. T. 23. f. I. 2. T. 29. f. 4. Fl. Dan. T. 597. f. 2. Vall. Paris. T. 20. f. 9. Dillen, Hitt. Mufc, T. 30. f. I3I. Michel. Gen. T. 47.

Habitat in faxis.

25I. Lichen probofcideus - membranaceus peltatus reticulato rugofus cinereo fuliginofus fubtus levis pallidior' folio crenulato erofo; tricis turbinatis perforatis demum converiulculis gyrofis.

Umbilicaria probofcidea. Svet. Rugglaf.

Lichen probofcideus Linnai. Afz. Ad, Sc. Svec, 1788.

Lichen deuftus Weh. Lightf. Crantz. Neck. Meth. Hag. Leysf. Rooh. Tent. Allioni.

Iichen melentericus Gmel. Sylt. Linn.

I ichen mefenteriformis Facqu. Milc, Ehrh. Rufir. Murr. Sytt. Linn.

Lichen pullus facqu, Mifc. Dicks. Wither. Murr. Sylt. Linn.

Lichen Jacquini Gmel. Sylt. Linn.

Lichen exalperatus Gunn. Retz, Pr, 2. Omol, Sylt. Linn.

Umbilicaria rigida Hoffm. Deut. Fl.

Umbilicaria mefenteria Schrad. Spicil.

Umbilicaria corrugatalHoffm.Pl.Lich, \& Dent.Fl. Umbilicaria exafperata Hoffra. D1. Lich.

Icon, 


\section{$-148$.}

Icon. Hoffm. P1. Lich. T. 2. f. I. 2. T. 43. f. 47. Fl. Dan. T. 47 I. f. 3. Facqu. Nife. T. 9. . 3.5.Dill. Hif. Mulc. T. 30, . 118.119. Habitat in montibus elatis.

Obf. Variat fuperficie vix rugulofa, reticulata, rugofa, afpera, atgue per atatem foliols fana. monofis tola fere tella, unde fpecies waria Auliorsm condire; Nec differe viderur:

Lichen torrefacts Lighif. Fl. Scot. Wither, arr.

Linuarnum nofirum Lachenem Aubtores facule ownes non perroverwat; quin etram nomess E.jus neglexeresnt.

252. Licnen cylindricus - mombranacens peltatus glauco cinerafcens, fubus levis ochrolencus, folio lobato fbrillis marginalibus atris; tricis turbinat is perforatis denum convexiufculis gyrofis. Umbilicaria ryliadrica, Svet. Trutlaf. Lich n cyludicus Lirn. Afz. Ad. Sc. Sv, 7788. Lichen crinitus Lightf. Smith Fl. Lapp. Lim, 2. Chats. Isl Mohr. Fl. Dan.

Lichen correus Gurn. Retz. Pr. I. Latourette. Lichn probofcite us Hudi, Rez, Pr, 2. Liljebl. Wcinu. Ehrh, Wulf. Gesn. With. Murray \& Gm:el, Syit. Limn.

Then polythizos Web. Weis.

Umbilicaria probnfidea Sehrad. Spicil.

Unbilicaria crinita Hoffor. Pl. Lich, \& Deut. Fl. Icon. Godur. Crypt. T, I, A. Hoffon. Pl. Lich. T. 44 f. 1 -9. Jacqu. Mifc. T. 9. f. 2. Fl. Din, T. 47I. f. 1.2. Dillen. Hift. Mufc, T. 29. f. 116 .

I abitat in alpibus noftris lapponicis.

Obí. Licheris hajus nomen, licet oblivioni datum, utpoie prisizns a b. m. Linneo impofutum, detreEare religioni withi duxi, pracipue quum ex jas alla. 
allatis hujus of pracedentis fynonymis, fatis coms. pertum habemus, quam facile fit unaim cum al. tera permutare fpecsem, o fic quidem omnes confundere.

253. LICHEN Spadochrous - membranaceus rigidus peltatus cinereoglancus nigro punctatus fubtus ater hirfutus villis ramolis, folio plicato fub. lobato; tricis demum convexis.

Umbilicarsa Jpadochroa. Svet. Fnoklaf. Lichen fparlochrous Ehrh. Cr. exl.

Lichen glaucus Wefr. Act. Sc. Svec, I793. Lichen velleus Web. Neck. Meth, Retz,Pr.2, Gunn. Roth. Tent, Mohr.

Lichen polyrrhizos Linn? Crantz? Ach. Aca, Ac. $\mathrm{Sc}_{2} \mathrm{SV}, 1794$.

Umbilicaria cirrola Hoffm. Pl. Lich. Umbilicaria fpadochroa kioffm. Deut, Fl. Icon, Hoffm. Pl. Lich. T. 2. f. 3. 4. Achar. Nov, Ad, Ac. Sc. Svec, V. XV. T. 2. f. 4. Habitat in faxis.

Obf. Maculis ferrogineis interdum contaminatus. Variat etiam foliis luxuriantibus of fubtus nudis. - A L. velleo differt rigiditate colore fubtus atro ef tricis demum convexis complicatis. An hic vel L. pellitus vel L. hirfutus ve-

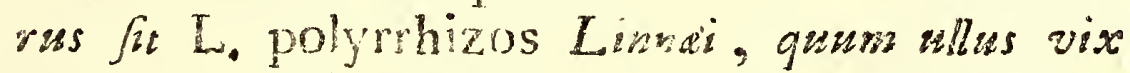
noverit, praftat fingulari alio nomine euss in. flgnire, graw, fisb vana lpe fynonyma certe val. de ambigua eir ircerta conciliandi, anfam confis. fronis multo majoris of erroris prabere.

254. LiChen pellitus - membranaceus peltatus Ixvisfimus fufco aneus fubtus ater hirfutus vallo ramofo floccofo, folio lobato; tricis deo mum fubglobolis sugofo gyrofis. 
Umbilicaria pellita. Svet. Luddlaf.

Lichen pellitus Ach. Act. Sc. Svec. I794.

Lichen hirfutus Weftr. ibd, 1793.

Lichen polyrrbizos Retz. Pr, 2. Liljebl. Huds. Lightf. Wither. Gunn. Fl. Dan. Neck. meth. Roth. Tent. Reich. Fl. Leys\%, Allion. Jichen fcalopodora Ehrh. Cr. exf.

Umbilicaria vellea Hoffm. Pl. Lich. \& Deut. Fl. Schrat, Spicil.

Icon. Hoffm. P1. Lich. T. 26. f. 3. El. Dan. T. 597. f. r. Dillen. Hift. Mufc. T. 30. f. I30. Achar. Nov, Act, Ac. Sc, Svec, V. XV.T. 3. f. \%

Habitat ad lapides \& rupes.

Obf. Variat polyphyllus, lobis crispis erectiufculis implicatis jubtus parce villoolis.

Hic promilcue jam Linnai L. polyrrhizos, jam Ejos L. velleus ab Auttoribus falutatur; ab hoc faltem toia die diverjus.

255. Lichen hirfutus - membranaceus peltatus molliufculus cinereus fubtus fufcus hirtus pilis fubfimplicibus, folio lobato margine inflexo; tricis hemisphæricis gyrofis.

Umbilicaria hirjuta. Svet. Ragglaf.

Lichen hirfutus Swartz. Retz. Pr. 2. Ach. Act.

Sc. Sv. 1794 .

Lichen polyrrhizos Wefr. Act. Sc. Svec. 1793. Lichen velleus var, A grifeus Liljebl. Sv, Fl. Umbilicaria hirfuta Hofm. Deut. Fl.

Icon. Achar. Nov, Act. Ac. Sc. Svec. V. XV. T. 3. f. I. Dillen. Hift. Mufc. T. 30. f, I 7 . Habitat in faxis \& lapidibus.

Obf. Variat folio papyraceo utringue cinerafcente fubtus nudo - Difinguitur a L. fpadochrøo habitu of Subftantia nec non colore $\sigma^{\circ}$ pilorum

figura. 
figura. - L. velleo proximus quidem of , fed differt habitu, folio rotunda, multo minori of frubiura recepraculorum.

256. Lichen velleus - membranaceus peltatus lavis latisimus utrinque cinereo fufcus, fubtus hirfusus villo ramolo, folio fubintegro plicato; tricis creberrimis planis plicis circinatis. Unkilicaria vellea. Svet, Fállaf.

Lichen velleus Limn. Liljobl. Ach. Act. Sc. Svec. 1794. Gmel. Sylt. Linn.

Icon. Achar. Nov. Act. Ac. Scient. Svec. V. XV. T. 3. f. 3. Dillen. Hitt. Mufc. T. 82, f. 5. Habitat in alpibus Lappon, \& mont. Veftro Gothia.

Obf. Folium oblongum inter Umbilicarias maximum, interdum fipitatum. - Tricis numerofis imis conftanter plaxis o harum plicis circularibus exade concentricis a religuis bene diffindtus. - Extra Sveciam borealem \& Canadom adhuc, quantum fcio, non inventa eft hacce fpecies, unde non mirum quod Auctorum fore newsini, excepto Dil. lenio, recte inounerit.

t L. velleiformis - membranaceus peltatus fubtus hire tus; peltis immerfis fubtus laccatis. - Lichen velleiformis Bellardi App. Fl. Pedem. (excl. fynon. Dillen. quod eft $L$. vellei ex Amer, feptentr.)

Umbilicaria? velleiformis. fax. alp.

NotA, Lichen carneus Wild, Roth. ad fungos referendus, eftgue Thalxphora carnea Schrad. Spicil. 


\section{$-152 .-$ \\ Tribus 16.}

\section{LoBARIA Svet. FlikLAFVAR.}

Folia membranacen fubcoriacea, lato lobata \& rotundaea; expanlo adicendentia, vaga, laxa, fibtus villofa.

Receptacula: Scutella fparla fubfesfiles, planiufculx pateriformes \& concave.

257. Lichen forobiculatus - fubcoriaceus expanfus rotundato jobatus lacunofus glaucovirefcens, fubtus ferrugineo nigricans villofus; glomerulis fubverrucolis; fcutellis fufcis margine pallido.

Lobaria fcrobiculata, Svet. Skrofeleld.

Lichen fcrobiculatus Scopoli. Swartz, Act. Upf.

V. IV. Retz, Prodr. 2, Lightf. Wither.

Lichen verrucolus Huds, Wulfo ap. Jacqu.

Lichen verrucarius Gmel.Synt.Linn,Schrad. Spic.

Lichen pulmonarius var. A. Scrobiculatus Lilj.

Lichen plumbeus Roth. Bot. Miag. \& Tent. Gmel. Syft. Linn.

Lichen Leers. Herb. 960.

Pulmonaria verrucola $H_{0}$ ofm. Pl. Lich.

Lobaria verrucofa Hoffm. Deut. Fl.

lcon. Hoffm. P!. Lich, T. I. f. I. Jacqu. Coll. 4. T. 18. F. 2. Fl. Dain. T. 1007. Rath. Bot. Mag. T. I. f. 2. Dillen. Hift. Mufc. T. 29. f. II 4. Mich. Gen. T. 49.

Habitat ad terram fupra mulcos, in faxis \& truncis arborum.

258. LiCHEN pulmonarius - fubcartilagineus expanfus reticulatus lacunofus finuatus, lobis truncatis, viridicaftaneus fubtus flavoferrugineus hirtus; fcutellis fubmarginalibus totis atrorubentibus. 
Lebaria pulmonaria. Svet. Lunglaf.

Lichen pulmonarius Linn. Reiz. Pr. 2. Liljebl. Hoffb. Scop. Lightf.Reich. Fl, Hag. Rcyg. Weis. Pollich. Mattuse. Gusn. Huds. Allion. Leys S. Wild. Pr. Neck. Delic, Rorh. Tent, Leers. Murray Prodr. Mall. Gmel. Tubing, Mohr. Doerr. Wigs. Scholl. Wither. Relh. Gmel. Sylt. Lim.

Pulmonaria reticulata Hoffm. Pl. Lich. Lobaria pulmonaria. Boffm. Deut .FI.

Icon Hoffm. 11. Lich. T. 1. f. 2. Diller. Mufc,

T. 29 f. I I 3. Moris. Hilt, T. 7. f. I. Mi. ehel. Gien. T. 45.

Habitat fupra arborum truncos in fylvis an. tiquis umbrofis.

Obl. Glomeruli in ma"ginibus etiam occurrunt.Var. vivipara in Roth. Mag. Bot. 2.T. l, f. 3.

259. LiCHEN perlatus - Rubmembranaceus expanfus rotundato lobatus lævis glauco virefcens fubtus niger fubvillolus; fcutellis elevatis concavis rubris.

Lobaria perlata. Svet. Bredlaf.

Lichen perlatus Limn, Liljebl. Huds. Pollich. Wulf. ap. Jacqu. Neck. Meth. Leers. Roth. Tent. Wither, Relhan. Gmel. Sylt. Linn. Lobaria perlata Hoffin. Deut. Fl. Icon Jacqu. Coll. 4. T. 10. Dillen, T.20, f.39. Waill. T. 2 I. f. I2. Mich. gen. T. 50, f. I. Habitat ad caudices arborum.

Obl. Interdum fupra aliquantum rugo/us, etiams fubtus lavis of nudinfculus. Scutelle fere pedrunculate majufcula, cava. - Differt a L. per. forato, cum quo neutiquam confundi debet, foucellarum difco femper imperforato of margine foliorum non ciliato.

K 5 
260. Liches herbaceus - fubmembranaceus expan. fus inuato lobatus lavis herbaceus, fubtus pallefcens fubtomentolus; fcutellis rufo fulvis. Lobaria herbacea: Svet. Gräsgronlaf.

Lichen herbaceus Huds. Swartz. Act. Upl. V. IV. Reiz, Pr. 2. Wither, Gmel, Syit. Linn.

Lichen latevirens Light. Fl. Scot.

Pulmonaria herbacea Hoffm. Pl. Lich.

Lobaria herbacea Hoffom. Deut. $\mathrm{Fl}$.

Icon. Dillen. Hift. Mulc. T. 25. f. 98. Hoffm,

11. Lich. T. 10. f. 2. Fl. Dan. T. I 124 .

Habitat ad arborum truncos \&t radices mufcis implexus.

Obf. Per fuccitatem cinereo virefcens, dein utringue ochroleueus, tandern fufous evadit, foutellis fus. scornfefcentibus.

26I. LICHEN glomuliferus - fubcoriaceus fubimbricakus expanilus finuato lobatus, finubus rotundatis, cinereo glaucus; fcutellis rufis glomerulisque atroviridibus.

Lobaria glomulifera. Svet. Skiftlaf.

Lichen glomuliferus Lightf. Fl. Scot.

Lichen laciniatus Huds. Hl. Angl, Wither, arr.

Lichen cumatiis Rutftr. Tisf.

Iichen mutabilis Ehrh. misf.

Lichen grandis Perfoon. Uft. Ann. d. Bot, St. I4.

Lichen amplisimus Scep. Carn.

Lichn laciniofus Gmel. Syft. Linn.

Lobaria glomulifura. Hoffm, Deut, Fl.

Icon. Dillen. Hift. Nufc, 1. 26. f. 99. Wichel. Gen. T. 46.

Habitat al radices truncorum antiqu. Fagi.

Obl:" Siccus per alignod tempus fervatus colorem. ochrolencum induit. 
* L. hottentottus - fubcoriaceus expanfus laciniato lobatus lobis incilo crenatis cinereo glaucus fubtus ater hirfutus; fcutellis elevatis iparfis margine ciliato.

Lobaria hottentotta, Cap. b. Sp. Afr. Thanb.

+ L, groenlandicus - funmembranaceus expanfus lobatus, laciniis obliquis rotundatis concavis, viridiglancus fubtus niger; fcutelis marginalibus fubrotundis. - Lichen grœnlandicus Gunn. Retz. Pr. 2. Fl. D.n. T. 466. Lobaria Grocnlandica. terr.

* L. perforatus - lubmembranaceus laciniatus inci. fo lobatus glancus fubtus ater villofus mar. gine ciliatus; fcutellis fubpedunculatis infun. dibuliformibus perforatis rufs.- Lichen per. foratus Wralf. ap. Jacqu, Coll. I. T. 4. Gmel. Sylt. Linn. - Lichen melanolecicus Wild. Bot. Mag. 4. T. I. f, 2. Gmel. Syit. Linn. - Lichen probofcideus Allion. Scop. - Li. chen laciniatus? Schrank. Gmel. Sylt. Linn. - Platisma perforatum Hoffm. H. Lich, T. 13. f. I. - Dillen. Hit, Mulc, T. 20. 1. 42 - 44. T. 82. f. 3 .

Lobaria perforata, trunc, arb.

+ L. ampullaceus - membranaceus planiufculus lobatus crenatus rufonigricans; fcutellis magnis globofis inflatis, - Lichen ampullaceus rats. ap. Jacqu. Coll, I. T. 4. fo 3 co. Huds. Murray \& Gmel. Sylt. Limn. - Platisma ampallaceum Hoffor. PI. Lich. T. 13. f. 20Dillen. Hift. Mufc. T. 24. f. 82. Lobaria ampullacea. pafc. montan.

\%. azalea-coriaceus expanfus crispus fordide! virens, verru- 
verrucis eonfluentibus nigsis, - Lichen azalex Schrank. Prim. Salisb.

Lobaria azalea. in Azalea procumb.

Nota, Tichen japonicus Thunb. Fl. Fapon. Hoffm. En. Lich. Gmel. Syft. Linn. folia Marchantze cujusdum prodit, nec verus Lichen efe.

Imbricarias iner $\dot{\sigma}$ Lobarias hasd quidem magna intercedit differentia; in has tamen folia nec protinus imbricata, negue cxiale rolulam formantia, fed vaga, diffuta atque lationa, fere coriacea \& jubtus denfius plersmgue villofa.

\section{Tribus 17.}

\section{Sticta Svet. PälsiafVar.}

Folia fubmembranacea lobata diffura adfcendentia, fubtus floccolo villoh.

Receptacula: Scitelle anticx laterales concavx, vel planx \& peltaxformes minginales.

Cyphella pofticx albx vel fallidx minutex inter lanuginem Sparfx. $_{\text {pax }}$

262. Lichen fylvaticus - membranaceus adfcendens finuato lobatus incilus fublacunofus ferrugineo viridis, fubtus fulvo nigricans villofus, cyphellis concavis albis; peltis marginalibus fuicis.

Stizla fyivatica. Svet. Skogslaf.

Lichen fylvaticus Linn. Retz. Pr. 2. Liljebl. Hads. Lightf. Wigg. Leers, Roth. Tent. Wulf. ap. Jacqu. Villars, Wither. Bellardi. Gmel. Syit, Linn. 
Lichen polyfcides Neck. Meth.

Pulmonaria fylvatica Hoffra. PI. Lich:

Peligera fylvatica Schrad. Spicil. Hoffm. Deut, Fl. Icon, Hoffm. Pl. Lich. T. 4. f. 2. ' Facqu. Coll. 4. T. 12. f. 2. Dillen. Hift. Mufc. T. 27. f. 10I. Mich. gen. T. 43 .

Habitat ad loxa \& montes mufcis immixtus in fylvis fterilibus humidis.

* L. damecornis - membranaceus adfcendens iviridicattaneus lineari laciniatus apicibus bifidis fubus fufco villofus, cyphellis concavis pallinis; fcutellis marginalibus rufis, junioribus pallide marginatis. - Lichen damæcornis Swartz. Prodr. - Platisma cornudama Hoffm. Pl. Lich. T. 24. f. I - 7. - Dillen. Hift. Muic. T. 29. f. I I 5.-Plum. Fil. T. 127. f. I. 2 .

Sticta damacornis, arb. Ind. Occ.

* L. laciniatus - fubcoriaceus expanfus viridi ca. ftaneus lobato incifus rapando fubcrenulatus fubtus fufco tomentofus, cyphellis concavis. pallidis; fcutellis fparfis planiufculis totis fufco rufis. - Lichen laciniatus Swartz. Prodr. Ic. ined. - Lichen lacerus Gmel. Sylt. Linn. Sticta laciniata. arb. Ind. Occ.

* L. gilvus - membranaceus adfcendens gilvus la. cunofus incifolobatus fubcrenulatus, cy phellis flavis concaviufculis; fcutellis fparfis nigris fubtus rutis.

Stitla gilva, arb. Cap. b. fp. Thunberg.

* L. tomentofus - membranaceus depresfus fuborbica- 
bicularis glaucovirefcens incifolobatus undulato crenulatus fubtus pallido hirfutus, cy* phellis concavis pallidis; fcutellis fparfis planiuculis obleure fuicis margine pallido. -.. Lichen tomentofus Swartz. Prodr. Ic. ined. Girsel. Sylt. Lim, Stutia tomentofa. trunc: arb. Ind. Occ.

* L. disfectus - membranaceus depresfus albido glaucefcens fublacunofus tinuato lobatus fubcrenulatus fubrus venolo tomentolus cyphellis planiufculis pallidis; fcutellis fpartis concavis rufis margine fubcrenulato. - Lichen disfectus Swurtz.Prodr. Ic, ined. Gmel.Syl, Linn. Sticta disfecta. arb. Ind. Occ.

+ L. fuliginofus - membranaceus fublepresfus cine: reo glaucus granulis fufco viridibus fuber it. nuato lobatus fubtus lpongiolo villofus, cyphellis concavis albidis; fcutellis ferrugineis margine pallido. - Lichen fuliginofus Dicks.

- Cr. Br. Gmel. Syft, Linn. - Lobaria fuliginofa Hoffm, Deut, Fl. - Dillen. Hit. Mufc. T. 26. 1. 100. Sticla fuliginoja. ram, arb. fylv.

* L. crocatus - fubcoriaceus adlcendens luteorufefcens finuato rotundatus margine undulato crenulatus flavo pulverulentus, cyphellis fiavis, - Lichen crocatus Linn. Swariz. Obf. bot. Dicks, Villars. Bellardi. Gmel. Syit. Linn. - Platisma crocatum Hoffm. Pl, Lich. T. 38. f. -3 -Dillen. Hift. Mufc, T. 84, f. 12. Sticta crocala. terr, ad rup. arb, mulc.

* I. flix - mombranaceus ftipitatus erectus glaucoviridis laciniatus laciniis incifo lobatis crenatis 
natis fubtus nudiufculus, cyphellis concavis; fcutellis fparfis rubris. - Lichen filix Szvartz, Meth. Mulc. 'T. 2.f. 2. Gmel. Sylt. Linn. Stibta filix. arb. Nov. Zeland.

\section{Tribus 18.}

\section{PeLtideA, Svet. SKINNLAFVAR.}

Folia coriacea expanfa depresfa rotundato lobata fubtus nuda ant tomentolo venofa.

Receptacula: Pelte marginales, in lobis plerumque elongatis adfcendentibus fesfiles, anticx aut poitica; rarius fpatia laterales \& immerfa.

* I. Poltis marginalibus anticis.

263. LiCHen wenofus - coriaceus cinereus futbus tomentolo venofus foliis fubrotundis divergen. tibus; peltis anticis marginalibus fublolitariis rotundis horizontalibus fufcis.

Pelidea zenola. Sver. Aderlaf.

Lichen venolus Limn。Retz. Pr. Hoffb. Liljebl.

Gunn Hud. Pollich. Lighef. Weis. Leers, Ehrh. Leysf. Mattufc, Neck. Doerr. Scholl. Mull, Rorh. Tent. Wigg. Mohr. Wither. Gmel. Sylt. Linn.

Peltigera venola Hoffm, F1, Lich, \& Deut. Flo Icon. Hoffm. Pl. Lich. T. 6. f. 2. Fl, Dan。 T. IIs5. Dillen. Hift. Mufc. T. 28. f. I09. 110 Michet. Gen. T. 44. f. 3. 5.

Hatirat ad margines viarum \& fosfarum locis umbrofis hunidis fylvaticis glareofis.

264. Lichen fpurius - coriaceus cinereus fubtus nudus 
nudus albidior venis concoloribus, foliis expanfis fubrotundis fublobatis, lobis pelta una alteraque antica adicendente rotundata nigrofufca terminatis.

Peltidea fpuria. Svet. Klippingslaf.

Habitat ad terram argillacean fubnudam.

Obf. Crefcit Jape imbricatuse infar L. venofi, cui magnatudine co habutu as amalis. Pelia in fingulo folio una, esiam dua, rarius 3. sut 4, 1310.

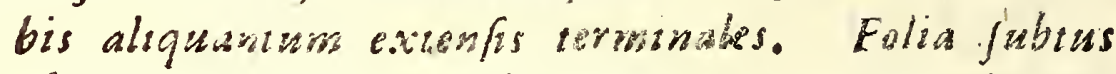
ahsque lanugine of fibrillis, venis parum elevatis nudis foncoloribus. Inter $\mathrm{L}$. fcucatum \&o $\mathrm{L}$. venofum medius, conjungens hos cum L. ca. nino ef. L. collino. Forte idem ac Dillonit Hifi. Mufc. T. 27. f. 102, A. B. Vix anters juniora Specimina L. canini nofter offert, guod de Juis pradicat. Cl. Dillenizs, licet fatear de hac Jpecie dubium withi esse.

265. LiCHEN horizontalis - coriaceus expanfus lo. batus glaber glaucovirefcens fubtus venolo lanuginofus; peltis, in lobulis elongatis; lesfilibus anticis fuborbiculatis horizontatibus rufis. Peltidea horizantalis. ' Svet. Plattlaf.

Lichen horizontalis Linn. Retz. Pr. 2. Liljebl. Lightf, liudss. Neck. Web. Gunn. Wuif. ap. Jacq. Leers. Schreb. Spicil. Wigg. Roth. Tent. Bellardi. Wither Gmel. Sylt.Linn. Peltigera horizontalis Hoffm. Deut. Fl. Icon, Facgu. Coll. 4. T. 16. Dillen. Hilt. Mufc, T. 28. f. 104. Michel Gen. T. 4, f. I. 6. Fl. Dan. T. $533 .-765$. pesfina. Habitat fupra faxa in fylvis inter mulcos.

266. Lichen caninzs - coriaceus expanfus rotundato lobatus cinerafcens fubtus venofus fibril- 
brillofus; peltis, in lobulis elongatis adfcen. dentibus, anticis fubrotundis rufolulcis.

Peltidea canina. Svet. Hundlaf.

Lichen caninus Limn. Thunb. Japon. Retz. Pr. 2. Hoff', Lillobl. Huds. Pollich Reich. Fl. Mattufc. Ailioni. Scholi, Deerr. Leers. Gmel. Tubing, Leys. Nurray Pondr. Whys. Roth. Tent. Scop. Nock, Web. Crantz. Hag. Regg, Lightf. Goonov. Gurn. Wulf. ap. Jacqu. Mull. Mohr. Wither. Relhan. Schrank. var, a. cinereus Weis, Gmel, Sylt. Linn.

Peltigera canina Hoffm. Deut. Fl.

Icon. Facges. Coll. 4. T. I4. F, I. Fl, Dan. T. 767. f, 2. Dillen. Fift. Mufc T, 27. f. 102. Moris. Hit. T. 7. F. I. Vaill. Paris. T. 21. f. 16. Blackv. T. 336 .

Habitat in montofis humidis \& unbrofis !ylvis fupra terram.

Obf. Varietas occerrie folics ampliganis, gue: Peligera amplisfina Hoffn, Deut. Fl.

267. Licuen aptatharus - coriaceus expanfus rotun. dato lobatio olaber viridis fuhtus fubavenius fibrillofus plag,a carulelcente, glomerulis fparfis fufcis; peltis, in Jobis attenuatis adfcentibus, anticis fubrotundis rubris.

Peltidea aphihofa. Svet. Torshlaf.

Lichen aphthofus Linn. Retz, Pr. 2. Hoffb. Liljehl. Gunn Leys. Huds. Lightf. Wulf. ap. Jaco. Murray Prodr. Scod. Pollich. Matturc. Crantz. thet. Neck. Allioni. Mohr. Wing. Hag, Leers. Scholl. Mull, Roth. Wither. Schrank. Gmel. Sylt. Linn.

Lichen caninus var. $y$ aphthofus $W_{\text {ais. }} \mathrm{Pl} . \mathrm{Cr}$. Peltigera aphthofa Wild, Pr. Hoffm, P1. Lich. \& Deut. Fl.

Icon 
Icon, Eoffor. M. Mich. T. 6. f. I. Facqu, Coll. A. T. T7. F1. Dan, T. 767. f. I. Dillen. Hitt. Mufc. 1. 28. f. 106.

Habitat fupra terram in fylvis acerofis.

Obf. Vuriezas foliss o glorserulis majoribus fubius nigricans, of:

Lichen verucohus Weberi. Roth. Tento Hag. \& Lichen veruciler Gmal. Syft. Linn.

268. LICHen polydadyy - coriacells expanfas digirato Jobatus glaber cinerco glaticelcens fubtus venolo fibrillofus; peltis, in lobis elon. gatis attenatis adfcendentil us, anticis fubro. tundis nigrofulcis.

Pelicidea polyaucilala. Svet. Treflaf.

Lichen polydactylos Retz. K'r. 2. Neck. Meth. Wulf, ap. Jacq. Leers, Roth. Tent, Gmel。 Syit, Linn.

Lichen canizus var, polydad. Web. Lights. Liljebl. Wither.

Peltigera polyductyla Roffon. R. Lich. \& Leur. Fl.

Icon Mofin. Fl. Lich T. \&. f. I. Jacqu. Coll! 4. T. I4. f. 2. Dollen. Hit. Nulc. T. 28. f 107.09

Habitat ad erran in fylvis \& dumetis,

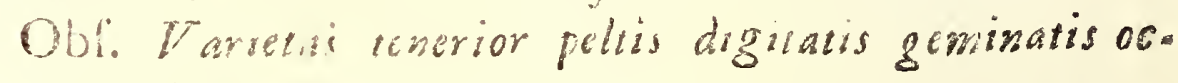
curvit, quic:

Lichen caninus pellucidus Weberi. Dill, l. $c_{\text {。 }}$ f. 108 .

269. Lichen collimu - coriacens lavis cinereo ru. fefcens ribus fibrillofus venosusque, foliis obrongs divergentibus incifolobatis crispis; peltis anticis fubfolitaris oblongis transerfalibus adlcendenibus nigro lpadicois. 


\section{$-163 .-$}

Pelide collina. Svet. Backlaf.

Habitat in collibus nemorofis umbrofis fupra terram.

Obf. I. canino $\mathcal{O}^{\circ}$ I. rufefcente multo minor at que toto habitu ab his or L. Ecutato dizerges.

* 2. Peltis marginaliules poficis.

270. LrCHen pularis - coriaceus expanfus pallido virelcens fubtus averius ad bafin nigricans, folits rotuntatis lobatis; $p$ this in lobis elon. gatis adicenduribus reminabus poficis maximis coccineis.

Peltidea polares. SSvet, Polarlaf.

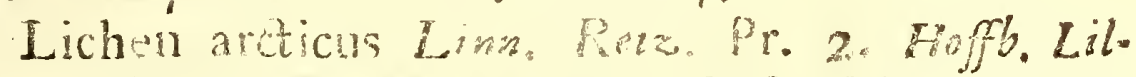

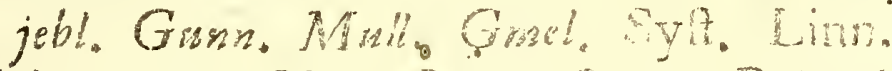

Ijichen antareicus Lisn. Gachot Buhardi. Gme! , Sylt. Linn.

Icon facqu. Milc. T. ro. f. I.

Habitat in campis fyluacis plerumque fub juniperis.

Obf. Etion in territ ad Circ. Polar. Antarizicum fuis occurrit cadom lpecies.

27I. LrCHen, refapimains - coriaceus adfcendens incilo lobatus plumbeo virefcens fubus ave. mius pallefcens fubvillofus; peteis marginalibus polticis reflexis rubris.

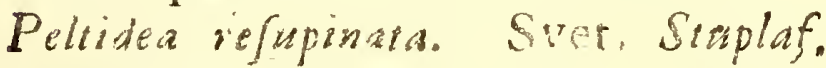

Lichen relupinatus Linn. Retz. Pr. 2. Hoffo Liljebl. Guns, Wriff, ap. Jacq. Lightf. Web.

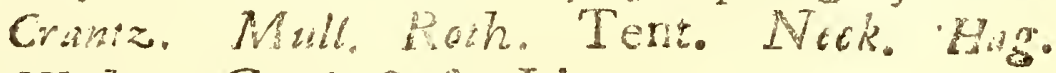
Whther. Grol, Sylt. Linn.

- Peligera refupinara Schrad Spicil. Pcligera tomentofi Hoffm. Deat. Fl. Icon. Jacgu, Coll. 4. T. 12 . I. Fl. Dan. T. 764. Dillen. Hit. Núc. T. 2?. Pf. 105. Michel. Gen, T. 44. f. 1.2. Ha: 
Habitat fupra terram ad lapides \& arborum radices.

Obf, Vin nis viariezates hujus funt:

Lichen lipponicus Liljebl. Sv. El. foliis cras. forthus margine crifpis fubous ardis fubrugofis, a:gus:

Pelite a papyracca Hoffmo. Deut. Fl. foliis who glabrs. Is. refupinatus Leers, Herb. C) halls in hac fpecie a sne nunguiam vifa, nec curm prizitys alles maralisze pallidis villo orbatis

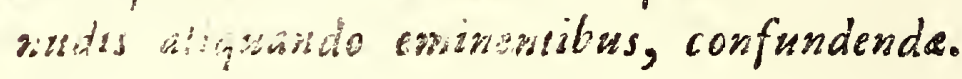

272. Tichen pailis - fulcoriaceus depresfus orbicharis fuisato lacieiatus unduatus crenatus vilifufcus- fubtus avengis nudiulculns fufcorigricans; peltis marginalibus pouicis reflexis baitis.

Felinda porilis. Siet, Vindwe

Habitat at laters montums.

Cbf. Folia fupra of ad margines glomerulis of pulvere fuliginofo haud raro adipera - Si spetie a precedente omaino non differat, autad tamen faus probabile vidcurr varietas adowodam forgularis eft, digna qua forrom defcributur.

* 3. Peltis lateralitus farjis E immerfit.

273. LICHEN agtaticus - coriaceus obcufe lobatus integer pallide virefcens; pelis lateralibus demun dilatatis folio majonbus fufco rufis margine inflexo.

Feltidea agratica. Svet. Vartnluf. .

Lichen aguaticus Limn Atez, Pr. 2. Boffb. Liljebl Geisn.? Gnitl. Sylt. Linn:

- Habiat fub dyua in páludibus mulcolis Upfalize ad Norrby.

Obi: Negne mini negue aliis maxime oculatis bo.

innicts 


\section{$-165 \cdot-$}

tanicis in loco fuo natali, ab Iil. Linseo indigi. tato, hunc Lithenem reperire fuccesst, neque dlibi." Fide fumm Viri inter frecantos Lichenes numerare tamen debus. Dillenii huc relata figu. ra T. 20 f. 44. L. perforati eft, de quo v. s. l. - L. faccidum in herbariis quibusdam $\mathrm{L}$. aquatici Limn. nonize etiain infcriptum aliguando capperius fum.

274. Lichen crocers - coriacens lavis depresfus cinnamomels labus venofus fulvus, lobis divergentibus linuatis; pelis fesflibus fparfis marginalibusque convexiufculis fpadiceis.

Pelridea crocka. Ever. Saffranilst.

Lichen croceus Linn. Reiz. Wr. 2. Hoffb. Lil. jebl. Huds. Gunn. Lightf. Neck. Meib. Wulf. ad. Jacq. Schran Curmark. H. Fr. Bellardi. Mohr. Wither, Gmel. Syft. Linn.

Peltigera crocea Hoffm. $\mathrm{M}$. Lich. con Hoffm. Pl. Lich. T. 41. f. $2-4$. T. 42 T. 45. Fl. Dan. T. 26\%. Jacg. Coll. 4.T. I I. f. 2. 3. Lim, Fl. Lapp. T. II. f, 3. Dillen. Hift. Mulc. T. 3 \%. f. 120.

Habitat ad rupium latera tenui terra tecta in fylvis \& campis lierilisfimis Lapponia \& Norr. landix.

275. Lichen faccatus - coriaceus depresfus fubim. bricatus rotundato lobatus fubcrenulatus cine. reo viretcons, fubtus albus fibrillofus; pelis immerlis fpartis nigro furcis.

Peltidea faccata. Svet. Sácklaf.

Lichen laccütus Linn Retz. Pr. 2. Hoffb. Lil. jebl. Web. Lechtf. Crantz. Neck. Gumn. Mattufc. Scop. Allioni. icers, Rorh. Tent. Wi. ther. Mohr. Gwol. Sylt, Linn. 
Lichen immerfus Hud. Fl. Angl. Eobaria faecata Hofm. Deut. H.

Icon Sowerby Engl. Eor. T. $289 . \mathrm{Fl}$. Dan. T. 532. f. 2. mala, Diller. T. 30. f. I2I. Mi. chel. Gen. T. 52. f. I.

Habitat in terra at radices arborum \& rupium qatera.

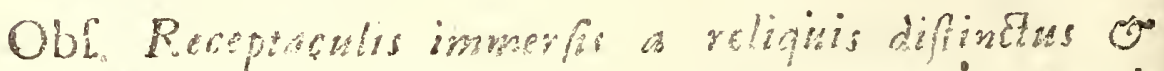

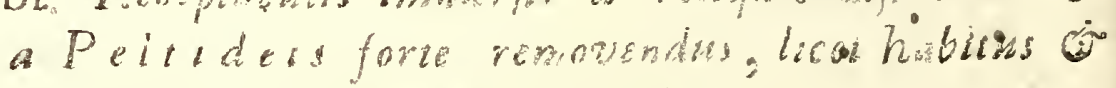

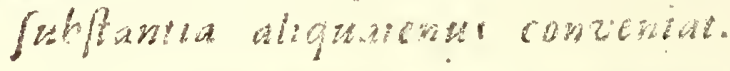

+ L, rufofen - concens repraming rufefens obture lobatus lobis matgine leflexis fmuatis chispis fubre villohs fubavenitus polis anricis rommatis aflcendentibus budis - Lichen ruf cens Wulf ap. Jacqu Coll. 4. T. 15. Nak, Moth. Roth. Tent. Laers? - Li chen rufus Grakl. Syth. Linn. - Lichen ca= ninus var. B rufefcens Weis. Web. Lightf. Wuther. - Heltigera nifefens Hoffm. Deut.

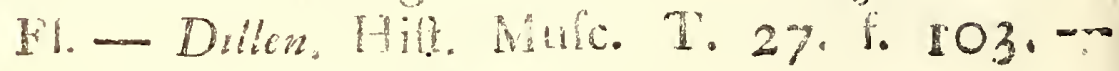
Michel. Gen, T. 44 , 2. Pellides rufefens. terrofylv.

* 1. foutan - coriaceus cinerens lubens nudus, folis lobatis multindis; pela mareinali antica bufefente. - Licher fcutatus Wyalfo ap. Jacq. Coli 4. T. 1\%. t. I. Docks. Peliadeafoulatio cortotrunc, arb.

\% L. coculentus - fuhcoriaceus albus extus rugofus cinerdicens; peltis prominulis inmerfis exca. vatis - Lichen efculentus Pallas It. T. I. f. 4. Gmel. Sylt. Lim.

Peludea? efculenta. inter lap: in mont. calc. 
Nota, Delitidec ommes, gue receptacsela "marginalie antica gerunt, grafie varuetutes vel modifications

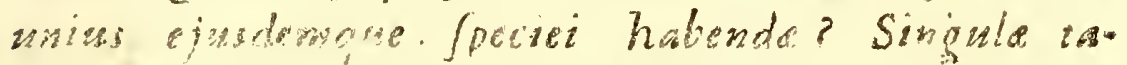

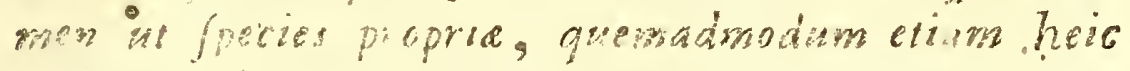

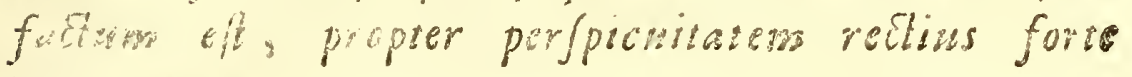
proponstatist.

\section{Tribus is.}

\section{PLATISMA. Suet. RRUSLAFVAR.}

Folia membranacea irregularia vaga, depresfa \& adlceno dentia, Aexuofa, inzequaliter laciniata margine nón ram so Aublacero crispo ex pulverulento, íupra \& luberis fere omnino minda.

Receptacula: Scutella elevata pelexformes. Gloneruli farit marginales.

176. LicheN glancus - membranaceus glaucus jubtus higer uninge levis, lolis adicendentibus complicatis mequaliter laciniatis crispis; fourellis furris concers rubicundio.

Platisma glancim. Sret. Isgralaf.

Lichen ginueus Linn. Retz, Pr. 2. Hoffe Lih jebl. Scop. Hosds. Web. Gunn, Wwalf. ar. Jacq. Ligher. Leers, Weis, Pollich Leysf. Allioni. Horm. Emm, Reich. D. Cratz. Neok. Wigg. Wid. Prolr. Roh Tunt, Gmel. Sylt. Lim.

Loharia glauca Hoffm. Deat. Fl.

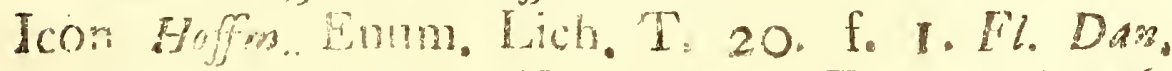

T. 593. Dillom Hit. Mulc. T. 25. F. 96. Mich. Kent. T. 50, f. I. Maris. Hit. T.7. f. 4. Vall. Par. T. 21. fi I2. Jargu, Coll. 4. T. 29. $\frac{5}{2} 2$.

$\mathrm{Ha}$ 


\section{$-168 .-$}

Habitat in truncis \& ramis arborum prafertim Belula albat \& ad faxa.

Obf. Variat totus nigar of fublus cinerens macn: lis difforminus atris, parumgue fibrillofus, nes non foliorsm lubis erectes, fubrotundis, plicatis, undulaus, tensiter laciniatis fiosbriatis, margine of Juperficie pialverulentis. Laciniarum apices interdas inflato fornicati, turbinati.

277. Lichen juniperinus - membranaceus pallideflavus fubtus flavis imus utrinque levis, foliis adfcendentibus complicatis lacero laciniatis crispis; fcutellis plano convexis badiis.

Platisma juniterinum, Svet. Enelaf.

Lichen juniperinus Limn. Retz, Pr, 2. Hoff'. Liljebi. Gunsa Huds. Lightf. Leers. Leys. Hoffis. Erum, Wulfo ap. Jacq. Mull, Murray Prodr. Wigs, Roth. Tent. Gmel. Sylt. Linn.

Lichen nivalis var. B luteus Wel. Spicil. Squamaria juniperina Hoffm. 1l. Lich. Lobaria juniperion Hoffom. Dent. Fl. Icon. Hofm, PI Lich, T. 7, f, 2. Ejusd. En. Lich.' T. 22. f, I. Fl, Dan, T. 1004. Buxb. Cent. $T .7 .3$.

Habitat in abulculis pixtertim juniperinis.

278. Licren pimari - nembranaceus fulphureus fubius fravas utringue ixvis, fliis deprestis complicatis inærialicer laciniatis margine adfcendentibus pulverulentis flavis mis.

Platisma pinageti. Svet. Graturuslaf.

Lichen piratri Scop. Bicks. Sihrad. Spic. Gmelo Sylt. Linn.

Squamaria piraftri Enffm. Pl. Lich.

Lobaria pinaftri Hoffm. Deut, $\mathrm{Fl}_{\text {. }}$

Icon, 
Icon. Hoffm. Pl. Lich, T. 7. f. I. Ejusd. Enum. Lich. T. 22, f. 2 .

Habicat ad radices Pini Abietis inque ramis Pruni fpinoliz o Juniperi.

Obf. Cum priorz fapisfome legi fed nunquam foutelliatsan vidi. Corte propago dooica L. Junipe. rini nec proprias fpecies.

279. Lichen fapincola - membranaceus lavis glaucolufus lubus pallidior lublacunofus, foins adfendentibus finuato lubatis margine erispis cinereo pulverulentis; fcutellis planiufculis caltaneis

Platisma fapincola. Svet. Gdrdfellaf

Lichen iepincula Ehrh. Hedvo. Dicks. Liljebl.

Hoffm. Enum. Gmel Syit. Linn.

Platisna fepincola Hoffm. Pl. Lich.

Lobaria repincola Hoffm. Deut. Fl.

Icon Hoffm. H. Lich. T. 14 f. I. Ejwd E. num. Lich, T. 17. f. 1. Hedw. Stirp. Cr. T. 2. f. $1-10$.

Habitat ad frepimenta lignea \& in ramis juniperi.

Obf. Indevidua alia margine ranitum pulverulenta nec fourellefera ubigue in fapibus occurrunt; alua folummodo fcutetitis confertrsformas obfenve caftanets obtecta in ramules juniperinus arides obfervantur.

* L. fallax - membranaceus tenuis glaucus fubtus candidus nigro fubmaculatus, toliis thitus complicatis laciniatis; foutellis terninalibus amplis fufcis. - Lichen fallax Weheri Drks. Roth. Tent. Gmel. Sylt. Linn, - Tichen fraudulentus Gmel. Syif. Linn, _ Platisma fallax Hoffm. PI. Lich. T. 46. f. $1-3 \cdot \frac{-}{50 .}$ 
Lobaria fallax boffm, Deut. Fl. - Dils. Hift, Nulc, T. 22. f. 58. - Michel, Gen. T. 37 .

Platisma fallax. rup.

\section{Tribus 20.}

\section{PHyscia. svet. BL ÄDDERIAFVAR.}

Folia membranacea, vel pro maxima parte cartilaginea, glabra, rigida, anguftiora, frofunde \& quấ ramofolaciniata, interdum pautulum latiora \& canaliculata lobato crifpa, cxipitota, erecta \& adfcendentia.

Receptaculo: Siutella fparfa \& terminales conçarx ac plano convexiulculix.

Glomeruli in quibusdam laterales marginalesque, nec nun foveolx in altis farinofx pasfim fuperficiales.

* 1. Foliis membranaceis vel longitudinaliter canaliculatis.

280. Lichen islandicus - membranaceus viridicaftaneus, foliolis adfcendentibus multifido lacini: atis canaliculatis ciliatis; fcutellis fesfilibus rlanis concoloribus.

Phyfria islandica. Svet. Islandslaf.

Lichen islandicus Linn. Retz. Pr. 2. Weftr. Act. Sc. Svec, 1794. Hoffb. Liljebl. Scop. Huids. Allioni. Lightf. Neck. Reich. Fl. Web. Crantz. Pollich. Mattujc. Hag. Weis. Lamark. El. Fr. Gort. Gerard. Gunn. Blackw. Leysf. Wither, Gifek. Reyg. Wigg. Murray Prodr. Mull. Roih. Wulf. ap. Jacq. Mohr. Ach. Act. Sc. Svec. 1797. Gmel. Sylt. Linn. Lichenuides islandicum Hoffm. Pl Lish. Lobaria islandica Hoffm, Deut. Fl. 
Icon, Fl. Dan. T. I55. 879. 7acqu. Coll: 4. T. 8. f. I. Hoffm. PI. Lich. T. 9. f. I. Dillen. Hitt. Mulc. T. 28. f. I I I. I i 2. Michel. Gen. T. 44. f. 4. Valent. T, 15. . 2. 3. Buxb. T. 6. f. I. -5 .

Habitat ad terram in campis apricis \& fyluis montofis acerofis locis fterilisfimis glareolis.

Obf. Variat magnitudine; laciniis anguftis of latioribus margine Jubnudis atque aculeato crispis. Glonseruli albi Sparfi rarius occurrunt. Recens bafi fanguineus, ficcus albefcens.

28I. Lichen cucallatus - membranaceus albus lavisfimus, foliis ereftis incilis tubulofo canaliculatis; fcutelis pofticis cucullatis fufcis.

Phyfcia cucullata. Svet. Kalklaf.

Lichen cucullatus Bellardi, Smith, Liljebl. Weftr. Act. Sc. Svec. 1794. Ach. ib، 1797.

Lichen ochroleucus Lamarck. Fl. Fr.

Lichen nivalis Weis. Web. Scop. Neck. Meth. Crantz. Vahl.

Lobaria cucullata Hoffmo Deut. Fl. Icon. Smith. Act. Soc. Linn. I. T. 4. f. 7. Dillen. Hift. Mufc. T. 21. f. 56. B.

Habitat ad teram in collibus fylveftribus aridis alpinis.

- Obf. Variat alous totus \&o pallide flavefcens. Ba. fis 'plerumque apud nos purpurafcens. Cum L. nivali olim fed perperam confufus.

282. Lichen nivalis - membranaceus albus lacunofus, foliis adicendentibus laciniatis crispis; fcutellis anticis cremulatis.

Phyfcia nivalis. Svet. Srólaf.

Lichen nivalis Linn, Retz. Pr. 2. Weftr Ad. Sc, Svec. 1794. Hoffb, Liljebl. Allioni. Willars. Weis, Web. Mohrovar, a. albidus Wé. Ach, Act, Sc, Svec, 1797. Gmel, Sylt, Linn. $\mathrm{Li}$. 


\section{$-172 .-\cdots$}

Lichen candidus Lamark. Fl. Fr.

Lobaria nivalis Hoffm. Deut. Fl.

Icon. Fl, Dan, T. 227. Dillen. Hit. Mufc, T. 2I. f. 56 A. Linn. Fl. Lapp. T. I I. f. 1? Habitat in campis nudis fabulous ficcis \& iterilibus.

Obl. Variat totus allus. Ba/is etiam flave/cens. - Differt a Lo cucullato fatura multo minori, follis latioribus lacunofis $\mathfrak{O}$ vix canaliculatis, marginibus magis crispis, bafi flavicante nec un* quass purpurea, foutellis anucis, fapore. - Re. ceptaciala nunquan ipfe vodi, fed fide Willarfii jam charaleri adpolasi.

283. LICHEv zenellus - membranaceus cinerafcens decumbens, folioman lacinis apice ciliatis adfententibus ubuloin fornicatis obruís; fcutellis fesilibus lateralinus niyrocalis.

Phyicia tenella. Svit, Snutlaf.

Lichen terellus teb. Swartz. Aa. Upf. V.

IV. Weft. Act. Ac. Sc. Svec. 1794 Scop.Allioni. Relhan Schol, Roth, Tent. Ach. Act. Ac. Sc, Svec. 1797 . Gmel. Syt. Linn.

Lichen hispidtis Sihreb. Spicil. Wild. Prodr.

Scop. I. Reich. Fl. Leers. Retz. Pr. 2. Wulf. ap. Jacq.

Lichen ciliaris var, Huds. With. var. a, Neck. Delic var. $\beta$ Pollich var. $\beta$ abidns $W$ eis var. $\beta$ Lightf. var, $\beta$ tenelius Liljebl. Lichenoides hispidum Hoffm. Pl. Lich. Lobaria hispida Hoffm. Deut. Fl. Icon. Hoffm. Hi. Lich. $\Gamma$. 3. f. 2. 3. Mich Gen. T. 5o. Vaill. Par. T. 20. 1. 5. Wulf. ap. Jacqu. Coll. 4. F. 6.

Habitat ad corticem arborum, in fapibus \& faxis.

Obf. Species a L, ciliari diverfisfima; fatura enim mulso 
multo minor, foutelle laterales of fesfiles, lacinie ad apices plerumque latiores convexa \& ibidem tantum cilsata; neque color \& ireligua proprieta. tes eadem.

284. Lichen ciliaris -.. membranaceus viridi glaucus, fubtus albus, foliis adfcendentibus lineari laciniatis ciiatis canaliculatis; Icutellis terminalibas nigrocalis.

Phycoia coliaris. Svet. Hârkantslaf:

Lichen ciliaris Linn. Retz. Pr. 2. Wefr. Act. Sc. Svec 1794. Hoffb. Laljebl. Scop. Web. Gunn. Hods. Leys/. Allioni. Wild. Prodr. Hedw. Neck, Schreb Spicil. Leers. Hag. Reyg Wigg. Roih. Tert. Wrulf. ap. Jacq. Pollich. Relhan. Mattufc. Wither, Reich. Fl. Loes. Lightf Lamurk Fl. Fr. Scholl. Mull. var. a. viritis $W^{\prime e}$ s. Ach. Act, Sc. Svec. 1797. Gmel. Sylt Linn,

Lichenoides ciliare Hoffm. Pl, Lich.

Lobaria ciliaris Hoffm. Deut. Fl.

Icon. Hoffm, Pl Lich. T. 3. f. 4. Fl. Dan. T. 7.1 r. Facgu. Coll. 4. T. 13. f, 1. Dillen. Hift. Mufc. T. 20, f. 45. Vaill, Par, T. 20, f. 4 . Tournef. T. 325 , f. 5 .

Habitat ad corices arborum \& lapides.

Obf. Variat foutellis margene radeato fimbriato of nigrofufacis; foliis viridz nigrecantibus of fupra glomerulis punitiformibus nigins adjperfis.

285. Lichen furfuraceus - membranaceus cinereo pulverulentus fubtus violaceo niger, foliis laciniatis canaliculatis decumbentibus; fcutellis fparis ampullaceis rubris.

Phy/cia furfuracea. Svet. Gjallaf.

Lichen furturaceus Linn. Retz. Pr. 2. Weftr. Act. Sc. Siv. 1794, Hoffb. Liljebl. Husds。 Lighrf. 
Lightf. Neck. Mattufe. Leysf. Pollich. Reich. Fl. Crantz. Web. Hag. Weis. Wild. Prodr. Wither. Gort. Gunu. Leers, Allioni. Hedw. Scholl. Wigg. Roth. Tent, Mohr. Mull, Ach. Act. Sc. Sv. 1797. Gmel. Syft. Lian. Lichen ablinthifolius Lamark.

Lichenoides furfuraceum Hoffom. P1. Lich. Lobaria furfuracea Hoffm. Deut. Fl. Icon. Hoffm. Pl. Lich. T. 9. f. 2. Dillen. Hift: Mulc, T. 21, f. 52. Michel, Gen. T. 38. f. I. Buxb. T. 7. f. 1. 2. Barr. Ic $1277 . \mathrm{f} .3$.

Habitat in faxis \& arborum cortice in primis Betulæ alba.

Obl. Variat interdus erectus of foliis cinereo inca. nis fere glabris, cinereoque nigricantibus floccofo. furfuraceis.

* 2. Foliis fulbcartilagincis planis ant tercti compresfis.

286. LICHEN prunafiri - lubcoriaceus mollis albocinerafcens rugolo lacunofus fubtus albisfimus', foliis lineari laciniatis planis erectiufculis; fcutellis lateralibus concavis fufcis. Phyjcia prunaftri. Svet. Slanlaf.

Lichen prunaltri Linn, Retz. Pr, 2. Hoffb. Wefr. Act. Sc. Sv. 1794. Liljehl. Lightf. Hag. Mattufc. Reich. Fi, Pollich. Weis. Reyg. Wither. Scop. Neck. Allioni. Gunn. Scholl. Muil. Mohr. Kniph. Leers. Murray Prodr. Wigg. Wuld. Prodr. Roth. Tent. Ach, Act. Sc. Svec. 1797.

Lichen corniculatus Relh. Wither, (var. anguftif Dill, f. 54 ) Fr. II. I. 
Lobaria prunaftri Hoffm. Deut. Fly

Icon. Dillen. Hitt. Mulc. T. 2 I. f. 54. 55. A. Michel. Gen, T. 36. f, 3. Vaill, Yar T.2O. f. 7. II.

Habitat in arborum truncis \& fæpimentis ligneis. Obf. Variat laciniis longioribus of angufis apice biffilis acuminatis; folisgue in cappitem congeftis brevioribus apice oónifis emarginatis, of glomerslis Jparfis. Differt a religuis molitite.

287. LICHEN fraxineus - fubcartilagineus cinereus rugofo lacunofus, foliis lanceolato laciniatis planis erectis; fcutellis fparfis lateralibus marginalibusque pallidis \& concoloribus.

Phyla fraxines. Svet. Ajklaf.

Lichen fraxineus Linn. Thunb. Japon. Rete. Pr. 2. Hoffb. Liljebl. Weftr. Act, Sc, Sv. 1794. Schreb. Spicil. Huds. Lightf. Scop. Hag. Allioni. Scholl. Weis. Wild. Prodr. Leers. Gunn. Ley/s. Mattufc. Wulf. Borufs, Neck. Reich, Fl, Wigg. Wither, Lamark, Fl. Fr. Mull. Mohr. Roth. Tent. Ach, Act. Sc, Svec. 1797.

Platisma fraxineum Hoffm。 Pl. Lich. Lobaria fraxinea Hoffm. Deut. Fl. Icon Hoffm. Pl, Lich, T. is. f, I. 2. Dillew, Hift. Mufc, T. 22, f. 59, bona. Mich, Gen, T. 36. f. I. bona, Tourn. T. 325 . f, A, B. Moris. T. 7. f. 3, 4, 5, 9, 14, 19.

Habitat ad arborum caudices \& ramos inprimis Qnercus \& Fraxini.

Obf. Variat laciniis linearibus longisfrmis pendulis, proliferis \& luxuriantibus, latisfzmisque, Jcutellis per ataiem flexuofis, nigrisque.

288. Licuen fafligiatus - fubcartilagineus cinereopallidus lacunofus ciefpitofus laciniato ramofus 


\section{$-176 .-$}

fus ramis faftigiatis teretiufculis furfum incrasfa. tis ramoîsimisque attenuat is fubinermibus; fcutellis terminalibus fesilibus concoloribus,

Phyfcia faftgiata. Svet, Klubblaf.

Lichen. faltigiatus Perloon Ult. Ann. St. 7. Schrad. Spicil. Ach. Act Sc. Svec. 1797. Lichen roltratus mas Scop. Carn.

Lichen populinus Ehrh..Cr. exf.

Lichen calicaris var, $\beta$ Weis, Auctorum?

Lobaria populina Baffen. Deut. Fl.

Icon Achur. Nov. Ad. Ac, Scient. Svec. V.

XVIII. T. 9. f, 1. A $-K$. Dillen. Hitt. Mufc. T. 21. t. 55. B. T. 23, f, 62. Vaill. Par. $T$ 20. f. 6 .

Habitat ad corticem arborum annofarum.

Obf. Multismodis vartat, fcilucet: fubhimplex, crase Jus, fubclavatus, or valde rasnolus, attenuatus; foutellis lateralibus fed abidem femper terminalibus, fuberus interdum appendeculatus, urceclatis $\mathcal{O}$ plano couvexis. - Cum L. traxineo, $L$. calica.

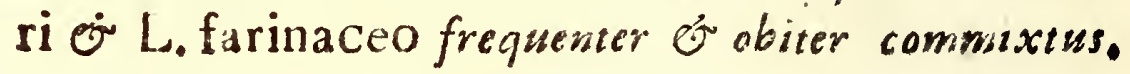

289. Lichen calicaris - fubcarilagineus pallidus compresfus lublacunofus erectus linearis ra. mofo pinnatifidus ; fcurellis pedunculatis con. vexis fparfis concoloribus

Physcia calicaris. Svet. Ndiluf.

Lichen calicaris Linnai \& Auforum? Ach。 Act. Sc. Svec, $179 \%$

Lichen fcopulorun Retz, $\operatorname{Pr}$ 2. Weftr. Adt. Sc. Sv. 1794. Ducks. Gmel sylt. Linn. Lobaria calycaris Hoffm. Weult. Fl. ? Icon, Achar. Nov Ac ivec, V. XVIII. T.9.

f. 2. A - H. Fl Dan. T. 959. f. 2.

Habitat ad rupes præcipu marisus.

Obf. Variat trunco fubioluario do celprrofo, laciniis

latioribus of teretzujculis, iniurvis, torulo/is.

- Ap- 
Appendices fub fourellis, vel potius ulimi lacinisrum apices inflexi, miniare corfoluns riota, fod pluribus Jpeciehus communis eff conformatio. Locus femper rapeffics. 'Sapor amurrsimus infar corticis Kinkina. His notis a L. fraxineo of faftigiato bene ctiam diffinguitur; proximus tamen tit hujgs variezanbus ramolas, at foutella nunguam terminales nic rami fajtgiati. - Speci. es cum L. faitigiato of farinaceo ab Auttoriribus femper confufa, of henc fynonyma Eorum dubsa.

290. Lichen farinaceus - carilagineus cinereo glaucus glaber lacunolus convexinfculis lacriato ramolus, ramis attenutis; ghmerulis con. vexis lateralibus; fonellis pedunculatis fparfis planis concoloribus.

Phyfcia farinaces. Svet. Mjollaf.

Lichen farinaceus Linn Retz. Pr. zo Weltr. A t. Sc. Sv. 1794. Hoffbo Liljebl Schreb. ISpicil. Huds, Lightf. Weis, Reich, El, Hag. Poilich. Mantufc. Walff. Borusf. Neck. Wither. Gunno Scholl Mohr. Mull. Leers. Leysf. Wigs, Wild. Prodr. Roth Tent, Al. lioni. Ach. Act. Ac. Sc. Sv, 1797. Gmel. Syit. Linn.

Lichen roftratus femina Scop. Carn. Lobaria farinacea Hoffm. Deut. Fl.

Icon Achar. Nov, Act. Ac. Sc. Svec, V. XVIIT, T. II. f. I. A - C. Dillen. Hit. Mufc. T. 23. f. 63. Vaill. T. 20. f. I3. I 4 .

Habitat in antiquis arborum corticibus. Obf. Variat paululum colore albo, glausco virefcenti \& pallido, fed infigniter fecindum formam laciniarum, linearabus foilicet of planing culis vel $\mathrm{M}^{\text {Subcanaliculatis ad axillas latioribus, apice. }}$ 


\section{$-178 .-$}

iensisfme frombriatis ac mulifidis, vel etians fur. furn latioribus omnimodo flexis, verrucolo fcas bredes of laciniato proliferis. - Glomerulis pre. cipue a L. tafligiato ot calicari differt. - Scis tella vix ab ulio antehac obfervate, nec defcripta.

291 Lichen pollinarius - membranaceo cartilagi. neus cinereus hinc pulverulentus cafpitofus lacero laciniatus; fcutellis fesfilibus fparlis albis margine \& fubtus virefcéntibus.

Physcua pollinaria. Svet. Dofilat.

Lichen pollinarius Wefrrmg. Act. Sc. Svec. 1794. Ach. ibid. 1797.

Lichen fquarrolus Perfoon. Un. Ann. d. Boto St. 14 .

Lobaria fquarrofa Hoffm. Deut. Fl.

Icon Achar, Nov. Act, Ac, Sc. Svec, V. XVIIf.

T. Il. $\mathrm{f}_{2}$ 2. A - F.V aill. Paris. T. $20 . \mathrm{f} 15$. Habitat ad parictes ligneos vetuftos \& caudices arborum grandxvarum nec non ad latera montiura.

Obf. $V$ riezas quadam latifolia in rupibus pracipue occurrens cadcm forte eft, ac:

Lobaria dilacerata Hoff'n. Deut. Fl. Dillen. T. 21. f. $55 . \mathrm{G}$.

Folia in nopra jpecie plerumque latiora quam in affinibus, plana, membranacea vel etiam fubcoriacea. - Scutelle majores fubtus rugole mar. gine elevato. Harsm forma atgue colore inprio mos diffingsitur a L. farin ace.

292. LICHEN polymorphus - fubcartilagineus pallide lutcicens erectiufculus compresius laciniato ramolus polymorphus; lacunulis fparis fariniteris; glonerulis fcutellisque congeltis terminalibus concoloribus. 
Phyfcia polymorpha. Svet. Mänglynnelaf. Lichen polymorphus Ach. Act. Se. S4ec. 1797. Lichen tinctorius Web. Hog, Ehrh, Cr. Lux? Roth. Tent. Grael. Sylt. Linn. Lichen calicaris var. Wefr. Act, Sc Svec 1794, Lichen fucoides Dicks. Gmel. Syit. Linn. Lobsria tinctoria Hoffm. Deut. Fl. Icon. Achar Nov. A\&t. Ac. Sc. Svec. V XVIII. T. II. f. 3. A - Z. Dillen Hiti, Mufc. T. 2I. f. 57. B. C. D. E. T. 22, f. 60. Habitat in faxis.

Obf. Vix ullus datur Lichen variationihus tam multis \& diverps obnoxius, unde determinatu $\sigma^{\circ}$

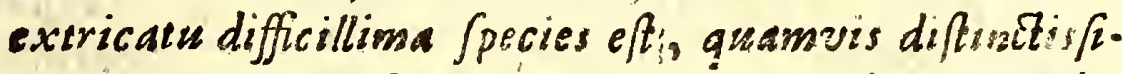
ona; atque fola fere comparatione varietatnm adeo disfimilisum sognitio ejus eruitur. V'ariat nempe

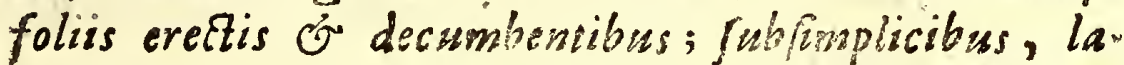
cinzatis, ramo/s, fimbriatis, proleferis, laceris of ramo/2ss frmis; lineartbus, lanceslatis, latioribus of filiformibus; plaxis, convexis \& cylindraceis; gla. bris es pulvere floccolo adjper/as; apición pulve. rulentis, obturis, globofis, acaminatis, laceris of glomuliferis. - Foveolis farinterss lacunofos of guarsudam varietatum glosmerulis teranizalibus, fapore fingulari ingrato nanfeofo es habizu pro. prio optime a religuis dignolcrutar. - Color per atatem in fordide fulvum vel ob/cure luse centern mutatur. - Madidus intra chartam fervatus am colore rubro tingit. - Species, fi now prorfass reglecta, haidenus tamen imperfecte deforipea to nota,

293. Lichen valpinus - cruftaceo cartilagineus "fulo vus capitolus erectus compresfus inxqual? angulofus ramolisfimus attenuatus. Physcia vulpiua. Sret, Taklaf. 
Lichen vulpinus Linn. Rotz.Pr, 2. Hoffb. Lilo joh. Gismn. Lughtf. Jacqu. Ehrh. Allion, Gmel. ruboig. Roin. Tent, Ach. Act. Ac. Sc. Svec, 1797. Gwel. Sylt. Linn.

Lichen aureus Lamark Fl. Fr. Lichen citrinus? Schrank Fl. Bav.

Lichen cirsicolorus? Gmel. Sytt. Linn,

Usnea vulpina Hoffm. Deut. Fl.

Icon Fl. Dan. T, 226. Jacqu. Mifc. T. IO. f. 4. Dillen. Hift, Mufc. T. 13. f. 16.

Habitat in tectis antiquis ligno imbricatis \& Ixpibus.

Ob1 Receptacula adhuc ignota. - Subftantia exzerna quale crufiofa, inzerdum pulverulenta, interior mollis bassbycina more phylcierum. Satis incongrue filamentoges antea adnumeratus.

294. LiCHEN farmentolus cartilagineus pallidus glaber lacunolus fiftulolus diffufus fubdicho. tonus, apicibus ramofis:mis capillaribus; fcutellis fparis insflibus virefcentibus margine albo. Phyfcia farmenrofa. Sver, Ranklaf.

Lichen larmicntolus Ach. Act. Sc. Svec, 1795, 1797.

Icon, Achar. Nov, Act. Ac. Sc, Svec, V. XVI. T. 8. f, 2. Dillen. Hift. Mufc, T. I I. f, 2. Mach. Gen. T. 39. f. 2.

Habitat in Lini fylveltris cortice arida, antiqua, $\&$ ad rupes.

Obf. Differt a L. usnea ramis infimis fiftulofis; fupremes capillaribus ramofissimis, joutellisque fesflibus cafio virentibus. 
* i。

* Li linearis - fubcartilagineus cinereopallidus ere. Qus gliaber linearis canaliculatus ramofo laciniatus; fcutellis marginalibus fubpedunculatis concoloribus glomerulisque terminalibus albis. - Lichen linearis Linn. Swartz. Meth. Mufc. Achar. Act, Sc, Svec 1797. Gmel, Syft, Linn. Phyfcia linearis. arb. Amer. fept.

* Li leucomelos - membranaceus albus diffufus li. nearis ramofus fubcanaliculatus coftis nigro ci. liatis; fcucellis fubpedunculatis cafio nigris margine radiato albo, - Lichen leucomelos Linn. Swartz. Obf. bot. T. II. f. 3. bona Diller. Hitt. Mufc. T. 2 I f. 50. mala. Achar. Act. Se Svec. 1797. Gmel. Syft. Linn. Phylcia leucomelos. arb. Ind. Occ.

\section{* 2 .}

* L. chryfophthalmus - membranaceus flavo aurantiacus cxlpitofus erectus linearis lacero laciniatus ciliatusque; fcutellis fulvis radiatis. Lichen chrylophthalmus Linn. Wulf. ap. Jacqu. Coli. T. 4. f. 3. Achar. Act. Sc. Svec. 1797. Gmel. Sylt. Linn, - Lichen aurantiacus Latourette Chlor. lugd - Platisma armatum Hoffm. Pl. Lich. T. 36. f. 4. Dillen. Hift. Mufc. T. 13. f. 17: - Varietas fcutellis margine integris nudis eft: Platisma denudatum Hoffno. Pl. Lich. T. 3I. F. I. quæ: Lobaria denuda: ta Ejusd. Deut. Fl.

Phyfcia ichryfophthislma. albor.

* L. complanatus - cartilagineus cxfpitofus erectus linearis laciniato ramófus planus pallidus pun- 
is marginalibus elevatis glabris albis; fcutellis fparis unceolatis concoloribis. - Lich a complanatus Achar. Ad. Se. Svec, 1797.

Phyfcia complanata, arb Lad. Occ, Swartz. Lejued.

* I, fuciformis - uembranaceo coriaceus albo cineralcens lubtomentolus erctiulculus planus linerilanceolatus pinnatifido laciniatus; futults glomerulisque marginalibus concoloribus, Lichen fuciformis Linn. Dicks. Achar. A tá: Sc. Svec, 1797. Gmel, Syit,-Lina. - Dollen. Hit. Mulc. T. 22, t. 6r. Phypcia fuciformis. arb.

* I. flammens - cartilagineo membranaceus flave. fcens cæpitofus erectus fubramsus fintulofus; fcutellis elevatis fparlis convexiulculis autantis, - Lichen flitmmeus Limn. Sutrtz. Math. Mufc, T. 2. f. 3, At. Med. Svec, t, T. 15. f. 3. Achar. Act. Sc. Sv. 1797. Grot. Syt. Linn. - Lichenoides flammeum Hof m. $\mathrm{H}$ Lich. T. 3. f. I. Phyfcia flarmea. arb. Cap. b. Sp. Afr.

* L. capen/is - cartilagineus flavus difufus teres ramofus fibrillofus apicibus capillaceis; cutel. lis concavis fulvis margine ciliato. - Lichen capenlis Linn. Thunb. Ach. Act. Sc. Svec. 1797. Gimel. Sylt. Linn. - Uinea capenis Hoffin. Pl. Lich. T. IO. F. 2. Phyfcia capenfis, arbor, ram, Cap. b, Sp.

* L. flavicans _- cartilagineus fulvus diffuins com. presfo fubangulatus ramolus attenuatns; fcutellis fesfilibus planis concolnribus margine integro. - Lichen flavicans" Swartz. Prodr, Ach. Act. 
A ct. Sc. Svec. 1797. - Lichen flavens $G m e l$. Sytt. Lino.

phyjcia flazicans, ram. arb. Ind. Occ,

* L. usnea - cartiagineus palidus proftratus line. aris complanatus ramolus attenuatus; fcutellis snarginalibus planis fubpedunculatis concoloribus, - Lichen Ufnea Linn. Achar. Act. Sc. Svec. 1797. - Dalien. Hitt. Mufc. T. 13. f: 14. T. 84. f. 10.

Phyfcia usnea. arb. Afre, Afr. Amer.

NotA. De Phyfoiis, prafertion Sveciam incolentibus; in Nov. Aliis Ac. Sc. Svec. (Vol.XVIII) fue fius egi; ut is guis Jpecierum defcriptiones carumque hifforiam, obfervationibus variis of figuris illufiratam, fuccinctague magis ratione propofitass

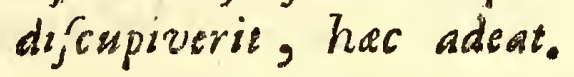

\section{Tribus 2I.}

\section{SCYPFOPHOR US.Svet. B ̈̈GARLAFVAR.}

Folia cartilagineo membranacea rigida fragilia fubimbri-

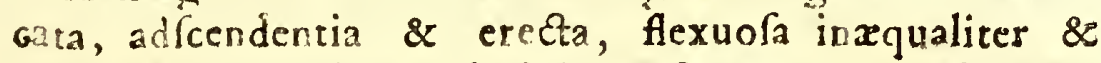
profunde utplurimum laciniata finuataque, vel etiam fublobato crenata; fupra virelcentia fubtus albida; lacilla tubulola fimplicia (per xtatem aliquando ta. mofa) fuperne incrasfata dilatata fcyphiformia claufa; difco concavo, producentia.

Receptacula: Tubercula convexa \& Aubgloboia fungiformia, ad margines fcyphorum; (rarius in ipfis foliis) sublesfilia, (inque bacillorum apicibus ramofis) ter. minalia, * 


\section{$-184$.}

* I. Bacillis fcyphiferis suprafoliaceis, foliisque majoribus erectiufculis profunde laciniatis.

295. LICHEN alcicornis - carrilageneus folis erectiufculis lobato incilis pallido virentibus; fcyphis fupratoliaceis minutis urbinatis; bacillis fimplicibus demum ramofis; tuberculis terminalibus minutis fufcis.

Scyphophorus alcicornis. Svet. Elghornslaf.

Lichen alcicornis Lightf. Relhan.

Lichen foliaceus Huds. Schreb. Spicil. Retz.

Pr, 2. Roth. Tent. Gmel, Syft. Linn,

Lichen pyxidatus var, \& Wither. Arr.

Lichen fquamolus var, B. 14. Scop. Carn.

Cladonia foliace Schrad. Spicil. Hoffom. Daut, Fl. Icon, Dillen. Hilt. Muic. T, is, f, I 2. A, B.

C. D. Vaill. Par. T. 2 I. f. 3. Michal, Gen.

T. 42. f. I - 2.

Habitat in ericetis locis macris \& humentibus. Obí. Folia ineerdum Jubpinnata. Scyphi parzi per atatem evarefcunt uti folia. Bacilla prime brevia forsolicia tanders ita excrefcunt elongantur of ramificantur ut ex facie vix eumdem Lichenem diceres.

Hujus etiam varietas forte eft:

Lichen phyllophorus Ehrharti \&o

Cladonia phyllophora Hoffon. Deut. Fl. bacillis in folida definentibus, fcyphis supra folia terminalia minutis Vaill. Fig. I. $c$.

296. LICHEN cervicornis _- cartilagineus foliis erectiulculis laciniato incilis cxliovirentibns; fcyphis fuprafoliaceis turbinatis demum proliferịs concoloribus, tuberculis marginalibus fesfilibus nigricantibus.

Schyphophorus cervicornis. Svet. Hjorthornslaf.

$\mathrm{Ha}$. 
Habitat ad terram \& lapides fupra mufos putrefictos.

Obf. Pulcherrima fpeces, forma of colore pergenams diffinta. Bacilla fimplecia minsta cylindreca, fod e fcyphorum difco planiafculo of margzine fape producuntur alia, femper tamen foyphifera of colore caliso indrata. Folia perfaliontia.

297. LicHen parechus - cartilagineus folis erectis lacinato incils dilue virefeentibus; feyphis obfoletis, bacillis turgidis ramolis, ramis terilibus porredis fubulatis, fertilium tuberculis terminalibus badiis.

Scyphophorss parechus. Svet. Hornbladslaf. Lichen turgidus? Ehrh. Cr. exl: Cladonia turgida? Hoffin, Dent. Fl.

Habitat in fylvis fterilibus \& ad montes locis apricis.

Obf. Scyphorum irregulariam vefrigia in folits obJervantur, at his denas abolutis bucilla elongatas o in morem L. uncialis ramsola occurrent, jed mages ventricofo, fepizs rupta o irregularia, ramis porreatis, Srevibus, fuhulatis, gutbusdam, apice tubercula minuta fimplicia, alis major conglomeriata fungefa, proferentibus. - Species ad. modism fingularas Scyphophoros Cladonits conjungens.

298. LiCHEN epiphyllus - cartilagineus foliis fubim. bricatis depresis adfcendentibus laciniatis mi nutis virefcentibus fcyphiferis?; tuberculis hemisphxricis lubfestilibus fufco nigris.

Scyphophorss epiphyllus. Svet: Kroolbladslaf. Habitat ad ligna truncorum emortuorum \& putrefcentium.

Obf. Folsatio of habitus fat furperque demonftrant M 5 hunc 
hasic esfe e foypheferorum gente; fed fayphos como pietos adhuc obfervare non licusit. - Tubercula in foliis jubjessflia ingue prima infantia guafi apice pertusa, aemum capstata fuborbicutata; color ravien formper idem. An tubercula periuja fey. phos vientusutia?

* 2. Bacillis Scyphiferis fubraiticalibus, folitis manutns fulimbricatis lobato crenatis.

209. Irchen pyridams - cartilagineus foliis minutis fubimbricatis lobato crenatis; bacillis brevibus obconicis cinereis, fcyphis dilatatis iubintegris; tuberculis narginalibus fufcis. scyphophorus pyxidarss. Svet. Trattlaf. Lichen pyxidaus Linn. Thunb. Jap. Retz. Pr. 2. Laljebi. Hoffb. Gusn. Gron. Doerr. Reich. H. Mulier. Mohr. Scholl. Pollich. Lighif, Reih. Schrank. Mattufc, Weis, Crantz. Hag. Allioni. Gmel. Sylt. Linn.

Lichen fimplex Roth. Tent. Gmel. Sylt. Linn, Lichen fquamofus II. E. b. c. Web. fpicil.

Cladonia pyxidata Hoffon. Deut, F!.

Cladonia limplex Ejuid. ibd.

Cladonia exilis Ejusd. ibd.

Cladonia incana Ejusd. ibd.

Cladoria verticillata Ejusd. ind.

Icon Dallen. Hift. Mufc. T. 14. f. 6. A. B. D. E. F. G. H. Vaill. Paris. T. 2 I. f. 5.7.8. Michel, Gen, T. 41. f. 3. K.

Habitat in ericetis \& fupra terram in faxis

Obf. Variat major es miner; humilis fimplicisfa. mans, vel e fcyphorum difco $\mathcal{F}^{\circ}$ margine prolifer; sistus of furfure, aliguando Squamuloso, tectus. Por Jenium tubercula majora eviadunt, 0 jam eft: I. ichen tuberculatus Hag. Hifl. Lich. Relh. quis: Clacionia tuberculofa Hoffm. Dent, Fl. Dillen. I. c. 
l. c. C. I. K. L. M. Vaill, T. $21 . f . n$. Mich. Gen. T. 4lo f. L. Moris. T. 7. f. 4.

Lichen polymorphus a pyxid. \& $\beta$ prolit. Roth. Tent.

Cladonia polymorpha Schrad. Spicil.

Cladonia prolifera Koffm. Deut. Fl. Mich.T. 42. f. P.

Cladonia marginalis Hoffm, ibd. Dill. l. c.I-M.

300. Lichen fimóriatus - cartilagineus folis minutis fubimbricatis lobato crenatis, bacillis cinerafcentibus elongatis cylindraceis, fcyphis denticulato crenatis; tuberculis minutis fulcis Scyphophorus fimbriatus. Svet. Nagglaf.

Lichen fimbriatus Linn. Retz. Kr. 2. Hoffo. Liljebl. Hag. Neck, Kundf. Wuif. Schuft. N. fr. Lightf, Mull. Crantz, Maunufc. Gurn. Allion. Scholl. Gmel. Sylt, Linn.

Lichen pyxidatus Reyg. Var. e. fimbr. Weis. Lichen fquamofus var. II. F. c Web. Lichen polymorphus $\gamma$ fimbr. Roth. Tent. Cladonia polymorpha Schrad. Spicil. Cladonia fimbriata Hoffm. Deut. Fl.

Icon Dillen. Hitt. Mulc. T. I4. F. \&. A. B. C. Vaill, Par, T. 2I. f. 6. 9. Mich. Gen, 'I'. 4 I. f. 5 .

Habitat fupra teram locis fterilibus fylvaticis $\&$ montofis.

Obf. Variat uti pracedens fcyphis e centro or mar. gine nec non bacilits ip/as proliferis, Juperficie nuda es pulverulenta.

301. LICHEN cocciferms - cartilagineus foliis minu tis fubimbricatis lobato crenatis, bacillis it nereo pallidis obconicis, fcyphis dilatatis futs integris; tuburculis fungolis coccineis. 
Scyphophorus cocciferus. Svet. Cocciomelliaf.

Lichen cocciferus Linn. Retz. Pr. 2. Hoffb. Libyobl. Hinds. Schreb. Spicil. Lightf, Reich. Fl. Mitll.Mohr. Gronou. Reth. Mattufc. Hag. Neck. Crantz. Scholl. Leers. Rorh. Tent. Gians. Alloni Mwray Prodr. Schrank. Gmel. Byti, Liasin.

Lichen pyxidalus var. f. coccif. Weis, Crypt. Lichen Guamoflis var. 10. Sop. var. II. ivl. a. Web.

Clactonia coccinea Hoffm. Deut. Fi.

Icon Dillen. Hit, Mulco $\Gamma$. I4. f. 7.A-M. Michet. Gen. T. 4!. f. 3. Vasll. T. 21.f. 4? Bockan Mutc. T. 104.

Habiat cum prioubus isclemque in locis.

Obf. Variat fimplex o s e margine centrogue fcyphorum prolifer. Thercula plerumgue fungofo, colore or magnitudine infogna, rat sfome per etatem oblourius fufca, fpeciem apprime duftingzand - Hujus eliawn loci funt:

Clarionia extenia Hojpm. Deut, Fl. bacillo lon. giori, fcyphis margine producto tuberculo/o val novis jeyphis tiberculofis prolifero. Vall. l. c. f. 4. - atgue:

Cladonia polycephaia Hoffon, ibd. bacillo Squa. molo foliofo fuperne dilataio, tuberculis atro. purpureis aggregalis zedto, Vaill, l. $c_{i} f .10$.

302. Lichen digizatus - cartilagineus foliis minutis fubimbricatis lobato crenatis, bacillis cinereopalli discylindrace is denum fubdivins, fcyphis ramolo digitatis; tuberculis coccineis.

Scyphophorus digizatus. Svet. Fingerlaf.

Lichen digitarus Linn. Retz, Mr. 2. Liljebl. Haffy, Gwizn. Huds. Allioni, Lightf. Relh. Pollich. Mull. Mohr. Leers. Roth. Tent. Grael. Sytt. Linn.

Li- 


\section{$-189 . \div$}

Lichen fquamofus var. II. M. e. $W e b$.

Lichen pyxidatus $\xi$ Wither. var, digit. Wis

Cladonia digitata Hoffm. Deut, Fl.

Cladonia coronata, Hoffmo ibd.

Icon. Dellen, Hift, Muic. T. I5. f. I9.

Habitat in fylvis macris.

Ob!. Singularis ob fcyphos digitato ramofos tandem evanefcentes, bacillis per atatem ramofis hasd raro jam foliogis of apice tuberculiferis.

303. Lichen deformis - cartilagineus folis minutis fubimbricatis lobato crenatis, bacillis pallido virefcentibus elongatis clavato ventricofis fubfimplicibus, fcyphis denticulatis irregularibus; tuberculis coccineis.

Scyphophorzus deformis. Svet. Fóslaf.

Lichen deformis Linn. Retz. P'r. 2. Hoffj. Hixds. Lightf. Relh. Matusc. Hag. Gunn. Gmel. Tubing. Alloni. Roth. Tent. Leers. Mohr. Gmel. Sylt. Linn.

Lichen cocciterus var, A. deform, Liljebl. Sv. Fl. Lichenfquamofus var. 4, Scop. var. I. 1. c.Weber, Lichen pyxidatus var. $\mu$ Wither. Arr, Cladonia deformis Hoffm. Deut. Fl.

Icon Linn. Fl. Lapp. T. II. f. 5. Dillen. Hift. Mulc. T. IS. f. I8. Michel, Gen, T. 4I. f. I.

Habitat fipra terram in fylvis denfis montofis.

ObC. Rarisfine prolifer, fed bacilla interdums ramos quosdam emittunt.

304. LiChen ventrico/us - cartilagineus foliis minutis fubimbricatis lobaio crenatis, bacillis cinerafcentibus farinofis elongatis cylindraceo ventricolis, fcyphis obloletis radiato ramolisfimis; tuberculis fuícis.

Soy, 
Scyphophorus ventricofus. Svet. Buklaf.

Lichen ventricolus Huds, Lighif. Gmel.Sylt,Lin. Lichen criftatus Rezz. Pr. 2.

Lichen fquamolus var. II. K. b $, y, W e b$. var. 15. Scop.

Lichen pyxidatus var. $\gamma$ criftat. Wois. var. $\gamma$ Wither.

Cladonia ventricola schrad. Spicil. Hoff $m$. Deuit, Fl.

Icon. Diller. Hitt. Mufc. T. 15. f, 17, A, B. C. Habitat in truncis arborum purefcentiun.

305. LICHEN radiazns - cartilagineus foliis nisutis fubimbricatis lobato crenatis, bacillis alboci. nerafcentibus elongatis cylindraceis fubfimpii. cibus, fcyphis digitato radiatis is tuberculis glomeratis fufcis.

Scyphophorus radiaicus. Svet.'Taidlaf.

Lichen radiatus Schreb. Spicil. Retz. Pr. 2.

Huds Roth. Tent, Hag. Gmel. Sylt. Linn. Lichen polyceras Schrank. Fl. Bav.

Lichen gracilis var. A. radiat. Liljobl. Sv. Fl. Iichen fquamofus var. II.G. Web. var. 4 . Scop Lichen pyxidatus var. $\zeta$ Wuher. Arr. Cladonia radiata Hoffm. Detut.

Icon Dillen. Hitt. Muic, T. 15. f, 16.

Habitat ad truncos putridos \& fupra terram ad lapides.

Obl. Toto habitu \& colore a L. digitato, fveir. tricolo of gracili differt. Variat lcyphis omai no laceris $\mathcal{O}$ objoleits, bacillis tennioribus, cras. foribus atgue lubclavatis. Tubercula rartus ob. Servantur glomerata vix unguam fimplicia. IHujas loci ctiam funt:

Cladonia polyceras Hoffin. Deut. Fl. bacillo fimplici, tuberculis Scutelliformibus, prolifero. 
Cladonia furcellatoradiata Hoffm. ibd. bacillo fimplici maximo furcellato ramoso Subulato cy. lindraco prolifero of tuberculifero.

306. Lichen gracilis cartilagineus foliis minutis fibimbricatis lobato crenatis, bacillis fulcovinentibus elongatis filiformibus fublimplicibus unbulatis hyphiterisque demum ramofis, icyphis dilatato turbinatis fpinofo denticulatis; tuberculis badiis.

Scyphophorus graetis. Svet. Stängellaf.

Lichen gracilis Linn. Retz. Pr. 2, Liljebl. Hoffb. Biuds. Neck. Lightf. Crantz, Pollich. Mattrefe. Hag. Allioni. Scholl. Leers. Schreb. Spicil. Rorh. Tent. Nohr. Gmel. Syft. Linn. Iichen fquamofus var. II. L. Web. var. I. Scop.

Lichen pyxidatus var. w Wither, var. h, gracilis Weis.

Lichen fimplicisfimus Schrank, Fl, Bav. Lichen polyceras ibd.

Cladonia gracilis Hoffm. Deut. Fl. Icon. Dillen. Hift. Mufc. T. I4. f. I3. Moris hit. T. 7. f. 6. Michel, Gen, T. 4I. f. 3. 4. 5 .

Habitat in fylvis fterilibus petrofis fupra terram. Obf. Variat fimpiex fubulatus \& fcyphifer, fcyphis longe destuculatis radiatis, radiis ipfis apice tuberculiferis, margincque fryphorum tuberculis minoribus fubpedicellatis fimplicibus coronato vel etiam prolifero. Bacilla per atatem more Cladoni ar um ramificantur, fcyphi evane fcunt o ramuli ultimi tuberculis folitariis fubglobofis terminaniur. Hanc metamorphofin in figura fua optime expresfit Dillenius. - Specie vix diffinguenda eft: Cladonia hybrida Hoffm, Dewt Flo bacillo ob. 
foure fcyphiforo margine tuberculifero prolifero fubrslato.

307. LICHEN turbinatus - cartilagineus foliis minutis fubimbricatis lobato crenatis, bacillis ci. nereo virefcentibus elongatis cylindricis fim. plicibus fcyphiferis, fcyphis denticulatis turbinatis demum expanfo dilatatis planiafculis; tuberculis marginaibus fubfesilibus glomeratis fubfufcis.

Scyphophorus turbinatus. Svet. Trumpetlaf. Icon. Michel. Gen. T. 4I, f. 7. Ord. VIII.

Habitat ad terram in fylvis fupra faxa.

Obf. A pracedente videtur definctus; Bacilla quams in hoc crasfiora, nec umgram jubulata, plerum. gue nuda, rarias foliolis adfperfa. Scyphi initio turbinati, margins rensusfime dersiculatn, deratsm expanfi, tuberculss fungofis, pallode forcas, ba. diis er tandzon fufco nigricantibus, plerwimgne conglomeratis, majortbus, fesfrilbus, rarins ele. vatis, circuncirca eleganter curonali. Proltfer etiam hic eft precipue e margine. - A nofre forte non differt:

Cladonia dilatata Hoffon Dive Fl. bacillis JHe perne dilatatis radiato tuberculiferis.

308. LICHEN cornutus - cartilaginets foliis minutis fubimbricatis lobato crematis bacillis cinereo virefcentibus elongatis fimpliciuculis fubventricolis acuminatis icyphif risque, fcyphis cylindraceis; tubercu is fufcis.

Scyphophorus cornutus. Svet. Spitslaf.

Lichen cornutus Linn. Retz. P'r, 2, Hoffb.

Liljebl, Gunn. Huds. Relhan, Lightf. Allioni. Scholl. Mattufce. Hag, Retch H. Neck. Wulf. Schrift. Nat. ir, Muller. Mohr. Gmel. Sylt. Linn, . Li- 
Lichen clavæformis Schrank Fl. Bav.

Lichen fquanofus var. I, A. B. D. Wib. var. I4. Scop.

Lichen pyxidatus var. C. Cornut, Weis. var. $\lambda$ Wither.

Lichen polymorphus var. $\delta$ cornutus Roth. Tent

Clatonia polymorpha Schrad. Spicil.

Cladonia cornuta Hoffm. Deut. Fl. P1. Lich. Icon Dilen. Hitt. Muic, T, Is f. 14. A-E. Hoffin. Pl. Lich. T. 25 . f. I.

Habilat lupra terram m laxis a fylvis.

Obl. Vix unguam prolifer, of rarisfime fabdivifus;

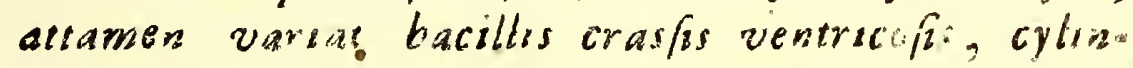

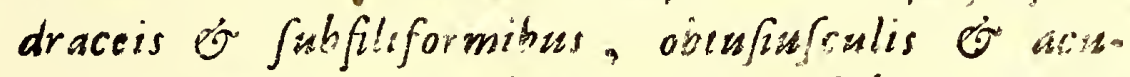
minains apiceque quafe uncinetis, glabris, fario nofis, fquamofis, verrucn/is of alperis, colore de. nigue albus, glancis, Jalphureis of fufcefcentibus.

309. Luchen filiformis - cartilagineus foliis fubimbricatis, incifo lobatis fubcrenatis, bacillis albo cinerafcentibus elongat is filiformibus Icyphiferis, fcyphis integris fubobloletis; tuberculis coccineis.

Scyphophorus filiformis.: Svet. Smallaf

Lichen filiformis Hisds Relhan. Gmel. Syit, Linn, Lichen tubifornis Ligh:f. Fl. Scot.

Lichen exiguus Neck. Delic.

Lichen Iquamofus var. II. E. a. Web.

Lichen pyxidatus var. $\xi$ With, Helv, var. C. cornut. Weis.

Lichen cornutus fcyphif. var, 7 Hag. Hit.

Cladonia tubxtormis Hoffm. Deut, Fl. (excl. Syn. Vaill.)

Icon. Dillen. Hift. Mufc, T. I4. f, 10. Michel. Gen. T.: 41. f. 6. N 
Habirat ad truncos putridos \& fupra terram in fylvis.

Obi. Quando tubercula, manifefaviur lcyphi fenfirn cvanefcum, of e masgine pasfim prolem enatsut. Bacsila fimplacia hused raro tuberculo termata

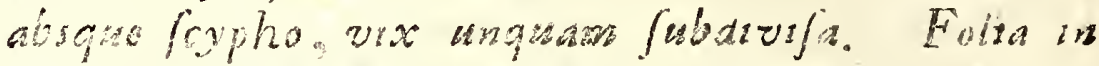

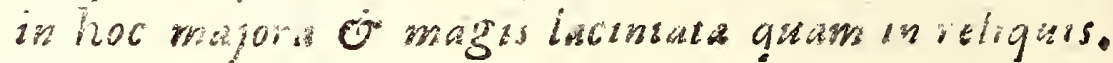

3!O. IRCHEN bellinforus - carilaginens folis mimatis lobmonicats lobaco crenatis, bacills pallio virentibus icobris elorgatis cylindram cers fubmoulis, feyphis termmalibus clavats; nuberculis minuminis conteris cocome Scyphophores bellediflorus. Svet. Blomepsluf. Habitat lup:a termon in montibus yly niun.

Obl. Species pulehorrima of ramisama, a relequis forma es habiu jatis diverja. Bucilla vix mila per proluficationerio fubramofos, foliolys lacinalus plerumgse lgramola, bafi fulva. Scyphe taneun zerminales tubcicula gerunt, que, torum mar. sinem obducertia or frequentia coloreque maxi. me speltabilia; corollularn plenam quodammodo referunt.

3:1. IICHEN fibula - cartilagineus foliis minutis fnbinbricaris lot a o crema is, bacllis incuis elongatis cylindraceis tup rne incrasiath sla. vatis, foypho ruberculis marginalibus fungofis fubfufis nbtecto.

Scyphorhor: flusla svet. Benlif.

Cintonia nbura Hoffon. Deut. Pl.

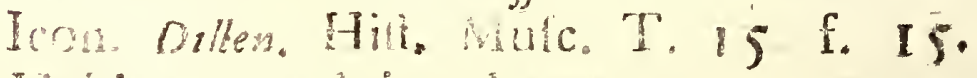

Thatite mo byivis al terram

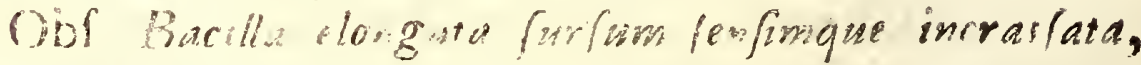

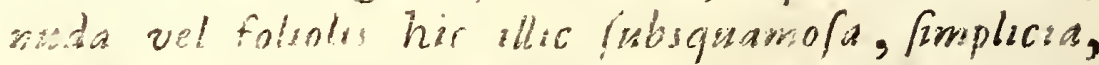
rawns audi hifida, terminata fcypho, quem fere obiegunt uborcula, forguga fes fira conferta. 
312. LiCHEN pleolepis - carilagineus foliis minu. tis ubinbricatis lobato crenais. bacills ci. nerafcenubus irregularibus tubulois, toliolis nulciitis lquamolis, lup in: rublivits per. viis ceisp's ramolo heris, feyplis obioktis; twhorculis fulets.

Scyphophorus plevlepis Svat, Fjalllaf.

Cladonia Gumbla Haffm. Wete. Fl.

Icon, Dilkn. Hit, Mufc. T. is f. 16 G. quam pro varietate Lo radiati habet, huic fimils. $\mathrm{H}$ ibirat cum prioribus.

Obl. Fere innumere hisus funt woldficationes of varietates. Species certe mixia, atque ext lasum Specserum varietaibus femelcentibus, aiso modo vin determinandis conflata, of hene pro lubutu ass camenda vel relinguenta. - Figura bictllorum erregulari, foliolic fapisfime lgitamolo exafperato. rum, aigue foyphis irregaloribur yel ommono de. ficientibus, ramulis crifpis, crifatis, lacerts, hic illic uberculiferis tantsm luperftutibus, potisfimum dignofcuntur hac referenda (pecimina. Forte etiam hujus loci funt:

Cladonia denticollis Hoffm. Dęut. Fl. perfora. - tor ramalis trsuncatis denticulatis.

Cladonia irregulnis Hoffm. $a b d$. perforata fim. plicinfcula fuperne ramofa ramis inequalibirs tubercusteferis. Dill. T. ts. F. 20 ?

\section{* I.}

* L. endivifolius - cartilagineus fllis incifolobrtis punatiouis contortis cripis latvovirefontrbas, feyphis inpratoliaceis minutis ip a fubim. plicrbus; tuberculis marginatibus atis. - Li. ch n endivitolius Dere. Cr. Brit. - Sis 10 . nia cormucopioius Hoffm, Deut. Fl. - Boeo. 
myces cafpiticius Perfoon Uft, Anṇ. St. 7.Michel. Gen' T. 42. f. 3. Scyphophorus endivifolizs. terra.

* L. ceracophyllus - cartilagineus foliis erectiufculis Jobato nocilis obutis palfido virentibus cornigeris - Lichen ceratophyllus Swartz. 'Prodr. Ic. ined. Gmel. Syit. Linn.

Scyphophorus ceratophyllus. terra. Ind. Occ.

$$
\text { * } 2 \text {. }
$$

$\div$ L. elongatus - cartilasinens, folis minutis fubimbrestis lobaro cenats, bacillis teretibus lon grams lublinplicibus albo cinereis, fcyphis obiolets tubulato denticulatis; tuberculis fu. fcis - Lichen elongatus Jacqu. Mife. Aultr. 2. T. II. f. I. * - Cladonia elorgata Hoffm. Tent. H].

scypiophorus elongatus. terra. Fret. Magell.

* L. monocarpus - cartilagineus folis fubimbricatis inción iobats, bacillis abis fubdiaphanis elongatis cylindraceis bafi apiceque dilatatis, fcyphis obioletis; taberculo lolitario coccineo. Scyphophorus monocarpus. Cap. b. [p. Thunh.

Nuta, Lichen comucopiodes Linnai, qui apud Dil. lemium Tab. 14. f. 9: eundem citat, Species admodum obfcura eft, nec facile diltu gsuam heic indigitare voluit Ill. Vir. Hoc etiam valet de religuis Audoram fyonymis ad hanc fpeciem re. latis; neque ex his omnibus collatis certior mihi comparata ef notitza. Varietatem proliferam -síe Sufpicor, fed cuidam ex jam enumsratis !peeitbus maxime propingua fit, ignoramus.

Quamvis fateamur, id quod fat ommino pro. babile videtur, plurimas inter jam allatas speci- 
es, Scyphiferarum familia, religuarum propagines es/e, tamen non onsnes ficut unius ejusdemque fpecici varietates habendas, ut quidam voluerunt, iam ex analy/s compertum eft, quaue obfervationibus prorfus indubiis. Hinc equidem putavi melius es'e atque utilius, tantum non 0. mnes, quos deprehendi nota quadam specifia magis conftante donstos, fingulaism proponere, quam in re adhuc dubia, ut mos aliquando $f u$. it, vana conjecture nimium fidencio, characteres horum Lichentsm flecci facere, varietatumque - indefinitarum multitudine of vemoriam \& Jaientiam labefactare.

Bafss omnium foliacea quidem eft, at temporis decurlu bacilla in plerisque, folie $f$ spectes, tam magna coadunt, ut potius ex ipfa terra, quam e foliis originem ducere videantur, quare etiem fubradicalia dixi; In aliis folia omnino evancfcunt, \& bacillorum forme adeo mutantur, ut juvenilem of fene/centem Lichenem pro diflinctis fpeciebus facile haberes parum attentus obfervator of inadjuetus collector. Hoc idem háeto de I. alcicorni, parecho, gracili \& q. J. r. Inde, $f$ quid video, omnis confutio of fere inexiricabilis error, in Jpecierum detcrminatione hujus Tribus, fubortus efl, nec, facile ewincendus. 


\section{- 198. - \\ Tribus 22.}

\section{HeI OP ODIUM. Svet. Fotlafvar.}

Folia cartilaginea rigida minuta 'fubimbricata erecturcula fincuato crenata fupra virelcentia fubtus pallidiora, bacilla fubfolida fimpliciufcula, fuperne parum dilacata vel exiam fubdivila apice tubercultfera.

Receptacuila: Tubercula in bacilis terminalia fungiformia majora fimplicia, gloncrata \& aggregata, margise fuomfiexa.

313. Lichen carrofus - carilagineus folis minutis fubimbricatis cremulatis, bachlis allovirelcentibus fubperviis cariolo cancellatis lcabris fuperne divilis; tuberculis atro pupureis. Fielupodium cariofum. Svet. Fratilaf Habitat in camp s therilibus glateofis. $\mathrm{Obl}$. Ob fayphos nallos vel faltem ommano imperfeElosinter Scyphophor os locumotounere non potef nec debet. Bacilla pasfim fimplacia iuberculo unico terminals.

3 I.4. Lichen fymphycarpus - cartilagineus f liis minutis lubimbricatis lobato crenatis, b.cillis cinereotufeis brevibus tubperviis luperne fubdivilis congentis tulcis.

Helopodium Jymohycarpurs. Svet. Druflaf. Lichen fymphycarpus. Eho h Crypt. ext. Lichen lutcus Foffm. En Gm Gmel. Sylt. Linn. Lichen arariciformis? Wulf. an jacqu. Cladoni fulca Schrad. spicil. Hoffim. Deut. Fl. Icon. Hoffm. Enum Lich. T, 8 f. 4. Dillen. Hilt. Nulc. T. 14 f, 2 . Jacgu. Coll. 4. T. 7. f. 3 ?

Habitat in ericetis \& Incis fterilibus, Obf. Tubercula in capunilum quali congefta non 
raro obfervantur, neque bacilla femper fubdivifa occurrunt, jed etzam fimplicua tuberculo unico capetulifornis terminata. - Licet nofter omajor fit, ron dutito eundern esfe curr Clac fulca Schrad. o Holfm. - Exir cataia apad Hofform. Michelia

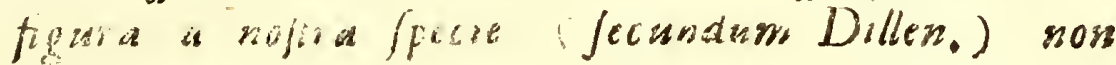
aiffert:

Liwen nacilentes Ehrh Cr. exf fi idem ac: Cludonia nuchente Hiffm Deut Fl bacillis

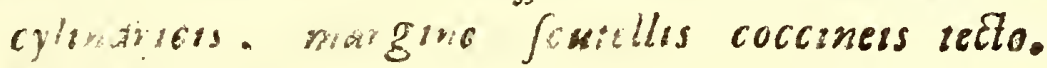
Mukiel. Gen 1. 42 f. 1.2

315. LrCnen benyes - cantilagireus folis minutisthis fuombricats cremiatis, bacillis palle. fientibus nubfitulofis inperse lubdivifis; tu. berculis aideretis dilute fulcis.

Helopodium botrytes. Svet Skaftlaf.

Lichen botryies Hag. Retz. J r 2. Liljebl. Wulf. ap. Jacg. Roth. Tent. Gmel. Sylt. Linn. - Boenyces abietinus Perfoon. Uft. Ann. d. Bot. St. 7 .

Cladonia botrytes Hoffm. Deut. FI. Icon 7acgu. Conl. 4. T. 4. f, 3. Hag. Hif, Lich. T. 2. f 9 .

Habitat ad muces putridos.

Obf. Tubercula fapissme fimplicia rorundaza coran vex 'rarius en:aqualia, fupra imbresfa; admodsum pallida, lusefcenua, rarius fujca. Bacilla intoro duss fimplicae:

316. Lrchen delucatus - cartilagineus follis minu. ticlinis lubimbricatis lacinato crenaris, bacil. lis conpresfufculis tuperne lubdivifis; tuberculis plobolis de num rigris.

Helopodium delicatum. Sver. Pysellaf.

J.ichea dicarus Ehrh. Ciypt exf.

Lichora piraiticus Hoffm. Euum. Retz, Pr, 2. Gmel. Syit. Linus. 
Cladonia parafitica Hoffm. Deut. Fl. Icon Hoffm. Enum. Lich. T. 8. f. 5 . Habitat ad cortices putrefcentes \& in terra fubltratis plerumgie mufcis.

Obf. Bacilla interdum ettam folioja, bi trifida vix ulera. Tubercula minuta conglomerata pri. mum carrea dein fufca of nirgra.

NoтA, Ob formam \& habieum omnino propriam feorfim \& fub fingulari Tribus jam memoratas 4 Species propofui. E bafi foliaces exfargune quidem earsom bacilla, quemadivodum in $S c y$. phophoris of Cladoniis ubtinet, jed ab il. les pracipue recedunt foyphorums ahfentia, quo. rum etians veftigia vix ulla facile detegenda. Neque cum Cladontis conjangere debui ob harumi firulluram prorfus diverfam. Bacilla e. nom in Helopodiis funt folitaria, brevia, crasja, fimplicia, Cuperne tantums faspliciter fubdivifa, plerumgue pervia; nec joyphiformia \& classa ut in Scyphophoris, terminala tuberculis majorihus, fungo/is, margine inflexis. In Cladoniis vero bacills elongata fenfim decrefcentia feu attenuata, fifulof., ramofissima eo fruticulo/a, ramulis terminalibos acuminatis, tubercula minuta, globofa. ... Helopgdi is proxime accedunt Boeomycides, wh horum crusfia non foliofa, bacilla fimplicissima ac intus fon lida funt. 


\section{- 201. - \\ Tribus 23.}

\section{Cladonia. Suet. Piplafiar.}

Folia cartilagineo membranacea minuta fubimbricata ereettufcula fublobico crenata, fupra virefentia fubrus albida, per atatem fere evaneicentia. Bacilia fi tulofa elongata atcenuata, fruticulofo ramola.

Receptacula: Tubercula fubglobofa, in ramulis baciliorum terminalibus accuminatis fesfilia, munta, fublolicarta.

317. Lichen uncialis - cartilagineus foliolis crenulatis evanefcentibus, bacillis rubulo s abo. palidis eredis ramois axillis perforacis, $1 \mathrm{a}$. mulis iterihbus brevistimis acuts, fertilium tuberculis ininutis fulcis.

Cladonia uncialis. Svet. Tugglaf.

Lichen uncialis Linn. Retz rr. 2 Hoffl, Liljebl. Scheb. Spicil Leers. Lightf Bab. Neck. Delic. Crantz. Hogen. Matulc, Gmel. Tubing. Huds. Wither. Wald. Reihan Lurn. nitz. Schrank. Rorh. Tent. Gurn. Allzoni. Mrill. Mohr. Gmel Sylt. Lin.

Cla io ii, u malis Hoffm. Deut. Fi.

Icon. Soverby Engl Bot. T. 174. Dillen. Hit, Mufc. T 16. f. 21. 22. Moris, Hitt, 1. 7. f. 7. Michel. Gen. T 40. 1. 2.

Habitat ad terram in lyluis fubhumi is \& 10 cis alpeftribus.

Obf. Varietates hujus funt:

Lichen ceramnilies Neck Delic. Allioni. qui:

Cladonia ceranoites Hoffon. Deut. Fl. hacilis diffufis, Juperne cinereo lubfufcis, ramis di. varicaris fuperne incrasfaits, ramulis faftigia. tis fubtruncatss. Dill. $l$ c. f. $2 l$.

Cladonia uncinata Hoffmo ibd. bacollis incanis N s

inde. 
inequaliter ramulofis radiatims uncinatis. $B$ :eriuncialis. Dill. l. c. f. 21. A.

Cladonia biuncialis Hoffm. $36 d$. bacillis fimpls: ciufculis pallidis nudis fponulofis, ramerlis brevisforis faftigiatis $2-3$ ftellatis, 'Dill, loc. f. 21. B.

Cladonia attenuata Hoffm. ibd, axillis dilatatis, rasulis furcatis attenuatis fellatis. Dill. $l_{0}$ c. f. 22. $\mathrm{H}$.

Magnutudo diverfa a femiunciali ad trisucioso lem; Color etiam varians, albus, incanus, virefcens. 7 am erectus eft, jam rami patentes, inaguales of falligiati, jam longiores, jam brevisfirme, uncsnati.

318. LICHEN pangens - cartilagineus foliolis crenulatis cvanticentibus, bacillis cinereis fragillis. fimis tubulofis cæipitoils ramofisfimis axillis imp iforatis, ramulis dichotomis divaricatis por cutis acutis.

Cladoria pangens. Svet, Sticklaf.

Habitat in locis aridis fterilisfimis fylvarnm.

Obf. In cafpitem denfum convexum collata o implexa Juni bacilla, in hac specie fragilisfima, dura. Tubercula adhuc non vifa. To:o habutu, axillis imperforatus of colore a L. unciali differt, cui alzas proximus.

319. LICHEN rangiferinus - cartilagineus, foliis crenulatis evanefcentibus, bacillis tubulofis abo cinerafcentibus cælpitofo ramofisfimis axillis perforatis, ramulis a pice deflexis, fertilum tuberculis globolis obfcure fulcis. Cladonia rangiferina. Svet. Rehnlaf. Lichen rangiferinus Lins Retz. Pr. 2. Hoffb. Liljebl. Gronov. W'eis. Pollich Lightf. Neck. Huds, Relh. Wuther. Lumnitz. Schrank. Hag. 
Hag. Leers. Gmel. Tubing. Leysf. Marray Prodr. Roth. Tent. Mattufc. Reich. Wl. Scop. Gisnn. Allseni. Mull. Schnli. Doerr. Gmel. Sylt. Linn.

Cladonia rangiferina Hoffm. Deut. Fl. Wild.Prodr. Icon Sowserby Engl. Bot. T. I73. Fl. Dasw. T.

180. 539. Dillen. Hift. Mufc. T. 16. f, 29.

30. Moris. T. 7. f. 9. Michel. Gen. 1. 40.

t. I. Tabers. Hilt. p. 11.98. f. G.

Habitat in fylvis montolis titerilibus.

Obl. Varietates hajus funt:

Lichen lylvaticus Allioni Fl. Pedem. qui :

Cladonia lylvatica Hoffm. Deut. Fl: 'bacillis brevioribus erestis minus ramofis', ramulis jub. fafciculatis, Dill $l$ c. f. 30.

Cladonia racemofa Hoifm, ibd ramulis tubercu. liferis racemofes exe itis.

Cladonia rangiformis $\mathrm{Hoffm}$. ihd. ramis divaricatis ramofisfomis, ramulis nudis breves/imis, furcatis erectis faßrgiatis. Noris. l. c. f. 0 .

Varietas alpeftris multo major ramulis im. plexis for concatenatis. Color in aliis rufe fcens, obfcure cinerafcens \& fulcus. Bacilla interdum foliolis minutis adjperfa.

320. LICHEN Jubbulatus - cartilagineus foliolis crenato lobatis, bacillis cinereo virefcentibus tubulolis dichotomo fubramofis erectis, ramis fimplicibus fubulatis, fertilium tuberculis badis. Cladonia fuóulata. Svet. Syllaf.

Lichen fubulatus Linn. Retz. Pr. 2. Hoffb. Liljebl. Huds. Pollech. Loes, Neck. Lightf. Schreb. Spicil, Leers. Roth. Tent. Mull. Mohr. Hag. Reih. Wither. Weis. Mattulc. Allioni. Leyss. Scholl. Lumnitz, Gmel. Sylt. Linn.

Cladonia fubulata Hoffm. Deut. Fl. 
Icon. Dillen. Hitt. Mufc. T. 16. f. 26. Tabern.

1197. 1. 2. Moris. T. 7. f. 4 .

Hableat in iylvis mufcis immixus.

Obi. Bacilla nuda vel etiam foliolis Jpar/is Jguamora

\section{Hujus etiam loci erit:}

Lichen cetaioides Hag. Haft. Lich. bacillis jubulatus becouter ramofis, ramulas non Jubdivifis.

32I. LICHen furcatus - cartilagineus foliolis crenula: is, bacillis albo cinereoque fulcis tubulo'is ramo is ramis adfcendentibus dichotomis biturcatis, fertilium tuberculis rufefcenlibus.

Cladorsa furcata Svet. Gaffellaf.

Licher tuicatus Huds, Schreb Spicil. Lighef:

Srholl. Relhan. Wither Reich. Fl, Hag.

Roth. Tent. Gmel. Syft Linn.

Lichen fubulatus var. A. furcat. Liljebl. var. $\beta$ Wiss.

Clat mir furcata Schrad. Spicil. Hoffm. Deut. Fl. Icon. Dilln. Hilt, Nufc. T. 16. f 27, Moris.

T. 7. f. I. p. 632. Vall, T. 7. f. 7. Habiat in jumperetis \& fylvis montofis.

Ois.: Varietates hujus funt:

Clatoria recurva Hoffm. Deut. Fl, ramis refle$x$ is atrenuatis foliofis glauco virefcentibus. Vall. l. c.

Clad rila furcato fubulata Hoffm, ibd. Fimplicisfould erecta fuperne ramoja furcato fubulata ultimis ramulis brevusfimis, Vall. T. 26. f. 7. Cladonia furcellata Hoffm. $b^{b d}$. bacillis albido pallefcentibus dichutomis longioribus ereflis flexuofis, Hag. Hift Lich. T. 2. f. 10. Praterea colore albo $\dot{\sigma}$ cinereo, bacillis fub-

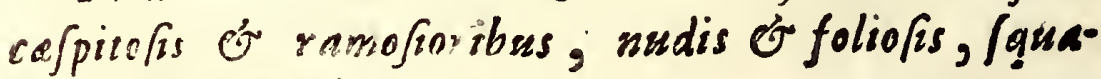
moso exalperatis variat. 
322. LICHEN Jpinofus - cartilagineus foliis crenu: latis, bacillis viridifufcis tubulolis vage ramofis tortuolis flexuofis fpinulofis apice dilatato fubpalmatis, ramulorum fertilium tuberculis fufcis.

Cladonia Jpinofa. Svet. To̊rnlaf.

Lichen fpinofus Huds. Lightf. Relhan. Hag.

Neck. Delic. Reich. Fl. Gmel. Sylt, Iinn.

Cladonia Ipinofa Hoffm. Deut. Fl

Icon. Dillen. Hilt. Mulc. T. 16, f. 25. Michel. Gen. T. 40. f. 5. Hag. Hilt. Lich. T. 2. f. II.

Habitat fupra terram ad colles \& montes fylvarum.

Obf. Hujos varietas eft:

Cladonia fpinefcens Hoffm. Deut. Fl. ramis trifidis, ramulis fterilibus brevusimis faftigrails.

L. fubulatum, furcatum \& ipinofium me. ras esfe ejusdem fpeciei varietates ju/picor licet ab Auctoribus diftinguantur.

323. LICHEN vermicularis - cartilagineus fubaphyllus, bacillis albis tiftulolis fubulato filiformibus proftratis fubdivilis; tuberculis? latera. libus dilute fufcis.

Cladonia vermicularis. Svet. Malklaf.

Lichen vermicularis Linn. Swartz, Dicks. Retz, Pr. 2. Liljebl. Wuther.

Lichen vermicellaris Gmel. Syft. Linn.

Lichẹn fubuliformis Ehrh. Beytr. 3.

Cladonia fubuliformis Heffm. Pl. Lichen \& Deut. Fl.

Icon. Hoffm. Pl. Lich. T. 29. f, I - 3. Disks.

Cr. Br. 2. T. 6. f, Io.

Habitat inter mufcos \& in locis graminolis prefertim alpeftribus.

Obf. Varietas hujus major, eft: 


\section{- 206. -}

Lichen tauricus Wulf. ap. Jacqu. Coll. 2. T. 12. fo 2. Schrank.

Cladonia taurica Hoffm. Pl. Lich. T. 34. f. 2. Ejusd. D. Fi。

24. Lichen nanus - cartilagineus fubaphyllus $c x$ fpitofus bacilis erectis cinereis bafi nigris fubfiltulofis ramolis, ramulis faitigiatis glome. rulato pulverulentis.

Cladonia mana. Svet. Lillaf.

Habitat in montibus terra tectis.

Obf. Species perexigua of diftincta. Habitus I. pafehalis. Bacella ad/cendentia confertis/ma ce. Spitem pulvinatum efficiunt. Ramuli undigne floccoso pulverulenti. hic illic of ad apices glome. rulis farinofis adfperfi. Receptacula nulla'deted a.

* L. aggregatus - cartilagineus fubaphyllus ; bacil lis flavis tubulofis ramofisfimis, ramulis m... ctis; tuberculis granulofo aggregatis fufco. nigricantibus. - Lichen aggregatus Swartz. Pr. Ic. ined. Gmel. Syit. Linn.

Cladonia aggregata. nont. Ind. Occ.

8. Lobulatus - cartilagineus fubaphyllus ; hacillis niveis tubulofis ramis timplicibus proftratis acutis apicibus recurvis, - Lichen tubulatus Villars Delph. 3. T. 55. Bellardi. Obf. An a L. vermiculari dittinctus? Cladonia tubulata. terr.

* L. Jpiculatus - cartilagineus fubaphyllus nivens; bacillis filiformibus tubulofis erectiufculis dichotomo fubramolis, ramulis fubulatis fimplicibus. 
Claderia fpiculata. mont. Ind, Occ. nubig. Swartz, ined, c. Icone.

Nota, Confimilis quidem eft Cladoniarum cum $S c y$ phophoris o Helopodiis origo o modus crefcendi, fcilicet quod o foliis bacilla erumpant: fed in his folia magis perffifentia, quando Cladoniar um fapisfime evane fcimt, of bacilla folitaria, tibera, quafi ex ipfa terra progenita, relinguuni. De catero mira eft inter $S_{c}$ yphopho ros, Helopodia of Cladonias analogia, que per fpecierum alias fat diffinctarmm varietates fibi invicem appropinquastes apprime maxifefatur. Foliaceorum denique of caulefcentiusm, quin immo cruftaceorum Lichenum, nexim of ciunt. 


\section{$-208 .-$}

III.

\section{LICHEN.CAULESCENTES.}

\section{Tribus 24.}

Stereocaulon. Svet. Busklafvar.

Caules ereeti folidi lignofi rigidi fxpe inxquales \& Icabridi, vel laves, rainofi: ranis inordinate effutis.

Receptacula: Scuteile fupra plaz vel etiam rotunda-

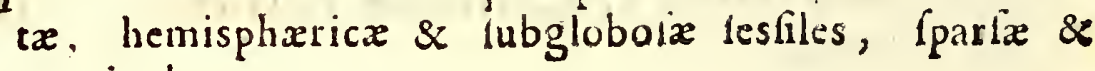
terminales.

325. LiCHen pafchalis - caulefcens folidus erectus fuffruticulofus granulólus $\mathfrak{c x}^{\prime}$ io cinerafens; fcutellis fparfis fubfesilib is marginatis demum convexis rugofis tufco atris.

Stereocaulon paschale Svel, Rislaf.

Lichen palchalis Linn Retz, Pr. 2. Liljebl。 Hoffb. Allion. Gunn Leers Weis. Pollich. Scop. Neck. Hag Scholl. Musl. Wulf ap. Jacq. Coll. 4. Roth. Tent. Gmel, Syit, Linn. Cladonia palchalis Wild. Prodr. Coralloides palchale Hoffm. Pl. Lich. Stereocaulon paichale Hoffm. Deut. Fl. Icon, Fl. Dan, T, 151. Hoffm. PI. Lich. T 5. f. 1. Dillen. Hift. Nlulc. [. 17. f. 33. P6iv. Gazoph. T. 65. f. 7. Sowverby Engl. bot. T. 282.

Habitat in campis arenofis \& fupra faxa.

326. Lichen confines - caulefcens folidus fruticulofus ramofus atrovirens, ramis teretibus 2pice incrasfatis pertufic. 


\section{$-209 .-$}

Stercocaulon? confine. Svet. Kuflaf.

Lichen confris $F l$. Dan. Mull. Deut. Mag. Icon. Fl. Dan, T. 879. f. 2. Mull, Deut, Mag. V: I. T. 23. f. 3 .

Habitat in rupibus marinis frepe inundatis. Obf. An potins Fuci fpecies? Fuco pygmæo lightf. Fl. Scot. T. 32 munor Etiam ab hoc aifinctus ramis teretubus nec quibusdam compresfis of fubpalmatis. - Loco crefcendi of apicibus hic illic altenuatis a noftro differt: Stereocaulon contine Hoffm. Dest. Fl.

† L. conder atatus — caulefcens folidus adfcendens fruticulofus soliolofus, ramis brevisfimis condenfatis; foutellis immixtis nigris turbinatis. - Stereocaulon condenfatum Hoffm. Deut. Fl. Stereocaulon? condenfatum. ericet. montos.

† L. obtufatus - ramulofus folidus aphyllis, ramulis teretibus aqualibus obtufis; fcutellis terminalibus nigris margine cinereo. - Lichen obtufatus F\%. Dan, T. I I26. f. I. Sterecaulon? obutatum. rup.

* I, ramulojus - caulefcens folidus fufruticulolus pallidus fcaber fublibrillofus, ramis vagis; fcutellis fubglobolis nigris parfis terminalibusque, - Lichen ramulofus Szarte. Prodr. Gmel. Sylt. Linn.

Stereocaulon ramulofuns. fax mont. Amer.

+ L. Sertularia - caulefcens folidus albus multifidus bafi ferrugineus, - Lichen fertularia Schrank Fl. bav. Gmel. Syft. Linn.

Stereocaulon? Jertularia.

O 
† L. guisquiliarius - caulefcens fruticulofus tenerrime ramulofus albus, tuberculis confertis la. teralibus atrofufcis. - Lichen quisquiliarius Leers herb. Roth. Tent. Gmel. Sylt. Linn. - Stereocaulon quisquiliare Hoffm. Deut. Fl. - Michel. Gen. T. 53. f. 8.

Stereocaulon quisguiliare.

\section{Tribus 25.}

\section{Sphat orhor us. Svet. Hy L SLA F VAR.}

Caules folidi rigidi cartilaginei laves fragiles fruticuloforamofi, ramis diffufis.

Receptacula: Cafclle terminales rotundate, pulvere nig'o fartex, demuin lacero-dehifentes concavx vacux.

327. LiCH:N globaferus - caulefcens folidus fruticuilolus cylindricus cinereo fufcus', ramis laxis divaricatis fibrillolis; ciftellis globolis. Spharophorus globuferns. Svet. Klumplaf.

Lichen globiferus, Linn. Retz. Pr. 2. Liljebl. Liphtf. Reich. Fl. Ehrh. Schrank. Neck. Sowerby.

Lichen globofus $\mathrm{Hudf}$. F]. Angl.

Lichen globiter Roth. Tent. Gmel, Syft. Linn. Coralioid $\leqslant$ s globiferum Hoffm. Pl. Lich

Stereocaulon globiferum Hoffm. Deut. Fl. Sphærophorus coralloides Perfoon。 Uft. Ann。 St. 7. Schrad. Spicil.

Icon. Sowverby Engl. bot. T. I I 5. Fl. Dan. T. 960. Hoffm. Pl. Lich. T. 3 I. f. 2 Dillen. Hilt. Mufc T, 17. f. 35. Mich, Gen. T. 39, f. 6 . 
Habitat in montium preruptis \& petris fylva. ticis atque in truncis Piai Abietis prope terram.

328. Lichen ferilis - caulefcens folidas caefpitofus cylindricus cinereo fufcus, ramis brevis. fimis confertis faftigiatis nudis.

Spherophorus fterilis. Svet. Skörlaf.

Lichen fragilis Linn. Fl. Svec. 2. Retz. Pr.

2. Liljebl Gunn Web Hag Neck, Ehrh. Lightf. Mohr, Roth. Tent. Gmel. Sylt. Linn. Lichen cxlpitofus Roth. Tent, Reich, Fl. Gmel. Syft, Linn.

Coralloides fragile Hoffm. Pl, Lich. Stereocaulon fragile Hoffm. Deut. Fl.

Sphærophorus tragilis Perfoon. Schrad. Spicil. Icon. Hoffm. Fl. Lich. T. 33, f. 3. Linn. Fl. Lapp. T. I I. f. 4 .

Obf. Ciftelle in hoc apud nos numquam obfervate. Specie forte diffinguendus a pracedente. adnucn. te $\mathrm{Cl}$. Perfoon \& Hoffm - Corpulcula minuta fubglobofa poro quali pertufa in rawns lis $\int p a r f a \mathcal{F}^{\circ}$ terminalio atra aliquando oc. currunt; receptacula vero non funt five ci. fellarum initia, fed corpora parafutica, quem. admodum in L. corallino alizsque videre con. tingit.

Cum L. fragili genuino, in Svecia adhuc son deteito, perperam aliguando confufus.

* L. fragilis - caulefcens folidus fruticulofo ramo. fus compresfus albidus; ciftellis terminalibus depresfis. - Lichen fragilis Linn. Spec. PI. Huds. Wuther. Facgu. Sowerby __ Lichen melanocarpus Swartz, Prodr, Gmel. Sylt. Linn. 
- Dillen. Hifr. Mufc. T. 17. f. 34. Sower. by Engl, bot. T. 114. facqu. Mifc, T. 9. f. 6. c.

Spharophorus fragilis. terra mont.

\section{Tribus 26.}

Cornicularia. Svet. Horntafiar.

Caules folidi rigidi glabri fruticulofo-czefpitofi ramcísfimi. Receptacula: Siutclla terminales planze, interdum ra. diatx, demum convexx torulofai \& inaquales "margine infexo.

329. LiChen trifis - caulefeens folidis nudus tereticompresíus fulcus cælpitaíus ramofus, rams adicendentibus faitigiatis fuperne nigricantibus; fcutellis terminalibus demum convexis fuiconigris, fubradiatis.

Cornucularia trifis. Svet. Tainglaf.

Lichen trittis Linn, Syartz. Retz. Pr. 2. Web. Schrad. Spicil. Ehrh. Gmel. Syit. Linn. Lichen rigidus Hoffm. Enum. Lich. Wulf. ap. Jacqu. Coll. 2.

Lichen radiatus Huds. Fl. Angl.

Lichen normoericus Gunn. Fl. Norv, (fec,

Cl. Vahl, in Fl, Dan, Fafc, 19.)

Lichen gagates La Mark Fl. Franc.

Lichen corniculatus Lightf. Fl. Scot.

Cornicularia tritis Hoffmo Pl. Lich.

Lobaria rigida Hoffm. Deut. Fl.

Icon Fl, Dan. T. II 26. optima. Web. Spicil. T. 5. f. I-3. Dillen. Hilt. Mufc. T. 17. f. 37. Hall, Hilt, T, 47, f, I, Ejusd, Enum.

T. 
T. 2. f. I. Gunn. F1. Norv. T. 2. f. 12. I3. 14. Hoffm. P. Lich. T. 34. f. I. Jacqu. Coll. 2. T. I3. f 5 .

Habitat in rupibus.

Obf. Scutella primum concava, rarius radiate. Hujus varistas, ramulis nerinque tuberculofo denticulatis, eft:

Lichen fucinus Gmel. Syfo. Linn qui:

Lichen fucuides $W$ ulf. ap. Jacq. Coll, 3.T. 12. f. 3 .

330. Lichen odontellus _ catilefens, folidus plano compresfus denticulato crispus nigm caftaneus cælpitofus ramofus, ramis diffufis.

Cornicularia odonteila. Svet.: Sporrlaf.

Lichen islandicus, var. tenusfimus Ehrh. Beytr, 5. var, nigricans Retz, Pr. Fl. Sc, 2. Lobaria tenuisima Hoffm. Deut. Fl.

Habitat in faxis \& rupibus apricis.

Obl.' Innocuis dentibus omni fere margine $\mathcal{O}$ ad apices ramorum crifpatst, nec proprse aculeatus. - Ut varietas tenella $L$. islandici olim habiuus, at cum illo es fequenie minime confunden. dus. - Icon Dillenii T. 17. f. 32. potius L. lanati junioris eft. - Scutelle apud nos adhuc non detecte. - Varietas hujus major, ramis crasforibus dilute caftancis, foutellis fpinofo ra. diatus demam convexis rufo fufcis, forte eft:

Coralloides aculeatum Hoffm. $\mathrm{Fl}$. Lich. T. S. f. $2: \%$

Icbaria aculeata Ejusd. Deut. El. (excl.synom.)

331. Lichen aculeatus - caulefcens folidiufculus teretiufculus crefpitofus nudus flexuofus fufco caftaneus, axillis compresfis, ramis atte. nuatis vagis apice furcato fpinulofis ; fcutel. lis terminalibus demum convexis fulcis. 
Cornicslaria aculeata. Svet. Spärrlaf. Lichen aculeatus Weberi! Ehrh, Liljebl, Schreb.

Leysf. Bellardi. Gmel. Syit. Linn. Iichen hifpidus Lightf. Gmel. Syit. Linn. Lichen caftaneus Leers. herb. Scholl? barb. Lichen fpadiceus Roth. Bot, Mag. Gmel. Sylt. Linn.

Icon Roth. Bot. Mag, 2. T, I, f, I, Dillen. Hilt. Mufc T. 17. f. 3 I ? Vaill. T. 26. f. 8. Mich. Gen. T. 39, f. 7: Moris, T. 7. f. II. mala.

Habitat in ericetis montofis.

Obf. Color variat pro diver $a$ atate, ad bafin tamen fere femper dilutior ibidemque rarius fufcefoens, ingue hoc fiatu wagnam prabes cum L. islandico afffinitatem. Rami plus minusque coms. pres/a \& lacunofi, interdum fubfifulofi of tela candidn molliori repleti. - Phy fcias cum Cor. niculari is connectunt: L. muricatus, odontellus, aculeatus \& ochroleucus.

332. Lichen muricatus - caulefceris fublolidus teretiulculus exectus rigidus fruticulofus nigricans flexuofus, ramis vagis; fcutellis terminalibus radiatis demum convexis amplis ine. qualibus badiis.

Cornicularia muricate. Svet, Broddlaf.

Lichen aculeatus Swartz. Act. Upf. V. IV. (excl. fynom, )

Habitat in locis liccioribus montofis prefertim maritimis,

Obf. $A \mathrm{~L}$. odontello sis aculeato omnino diver. fus videtur. Subftantia fragilis/rma. Caules $\dot{\mathcal{O}}^{\circ}$ gavai admodum teneri, ad axillas non compres/z fere sigri, bajî tantum fufcefcente. Habitus ferme fequentis. 
333. Lichen bicolor - caulefens folidiufculus erectus rigidus fruticulofis ater, fummitatibus cinereofufcis, ramis fibrillofis, difulis vagis attenuatis.

Cornicularia bicolor. Sver. Quaflaf. Eichen bicolor Ehrh. Cr, exf. Beytr. 3. Hann。 Mag. 9. Gmel. Sylt. Linn. Usnea bicolor Huffm. Deut, Fl.

$f$ abitat ad latera rupium inter mufces.

Obf. Species hac diffindivisma ad I. muricatum forma parum accedens, Jefe prodit funsmitatibus difcoloribus. Recepracula adhuc non detecta.

334. Lichen ochrolescus - caulefcers fubfolidus crecus teres lavis pallirlus futiculolus, ra. mis divaricatis apice furcatis nigris; glomem rulis verrucofis fparfis.

Cornicularza ochrolenca. Svet. Svartiopslaf.

Lichen ochroleucus Ehrh, Laljebl, Dicks. Lichen pallidus Retz. Pr. Fl. Scand. 2. Lichen fubvirefcens Gmel. Syft. Linn. Lichen flavidus $G m e l$. Sylt. Linn. Lichen fulphureus Olafs. Reif, Fl. Island. Lichen rigidus Villars Fl. Delph. Bellardi. Usne a ochroleuca Hoffm, Pl. Lich, \& Deat, $\mathrm{Fl}_{\text {. }}$ Icon Hoffm. P1. Lich. T. 26. f. 2.

Habitat in alpibus.

Obf. Ad primum intuitum facies $U s n \in a$, at nequa crefcendi modus nec iexiura eadem. Nulla essizas crufta externa corticalis, fed cartilaginea of nssta lum filum medullare, fed fubftantia intus bossbys cina. Phyfciis admodum propingua fpecies. Sorstelle adhuc forte incognita. Rami interdum tobi. pallidi apiceque concolores.

335. Lichas mufcicola - caulefcen folidus teres 
atrovirens cæépitofus ramofus, ramulis nodulo. fis flexuofis taftigiatis obtufunculis; fcutellis libus brunneis margine integro.

Cornicularia mufcicola. Svet. Klenlaf.

L.jchen mulcicola Snartz. Nov Act. Upf. V.

IV. Retz Pr, 2, Liljebl. Dicks. Ach. Act,

Sc. Sv. 1795. Wither. Gmel. Sylt. Linn. Collema ramulolum Hoff Icon Achar. Nov, Act. Ac. Sc, Svec. V. XVI.

T. I. f. 3. Decks, Cr. Britt. 2. T. 6. t. 9. Habitat ad montes intor \& lunra mufcos.

Obf. Habutus omnino Cornicularia quando ficcus. Madsfactus more Collemalum rubgelationfus evadit, utu etiam alue fpectes in hac Tribu.

336. Lichen lanatus - caulefcens folidus teres la. vinfculus fulconiger decumbens cxipitofus, ramis filiforaibus implicatis repetito dicho. tomis.

Cornicularia lanata. Svet. Ulllaf.

Lichen lanatus Linnei. Weftr. Adt. Sc. Svec. 1797. Retz. Pr. 2. Hoffh, Liljebl, Hadf. Gunn. Lightf. Crantz, Mateufco Roth. Tent. Mohr. Wither, Reihan, Gmel. Sylt. Linn. Lichen faxofus Gmal. Sylt, Linn.

Lichen jubatus var. $\beta$ lanatus Neck. Metho Iichen pubefcens var. $\beta$ major Weber Spicil. Usnea lanata Hoffsm. Deut. Fl.

Icon Dillen. Hit. Mulc, T. I3. f 8. minus bona. Habitat in lapidibus \& rupbus.

Obf. Hic, qui L. lanatus Linn ab initio cappio tofus, nunquam foutelliferus adhuc repertus eft; fed in varietate ejus minori, guam pro Ejusd. L. pubefcente male habuerunt nonnulli, interdum Jcutelle obfervantur plane \& concoleres. Hecce varietas, Specie vax diftinguen- 


\section{$-217 .-$}

da, tenuior, atro nitesss, ramulis initio fubionbricatis centrifugis demum tamen ut in iplo $\mathrm{L}$. Janato cespatoris, ingue alpinis potisfrisum pro. veniens, Jynonyma agnofcit fequentia:

Lichen lanatus Schrad. Spicil.

Lichen rigidus Wuif. ap. 7acq. Misc, 2.

Lichen reticulatus $W$ ulf. ap. Iacqu. Coll. 2. Gmel. Sylt. Linn.

Lichen pubscens Retz Pr。 2. Web. Lightf. Winlf. ap Jacq. Micc. 2, Gunn. Crantz. Roth. Tent. Mohr. Bellardi, Gmel. Syje. Linn.

Lichen laneus Ehrh. Cr. exs.

Usinca lanea \& U. pubescens Hoffm, Deut. Fl. Icon Jacq"o Mifc. 2. T. 9. f. 6. a f. 7. Ejusd. Coll. 2. T. I3, f 6. Schrad, Spicil, T. I. f. 6. Dillen. Hift. Mufc, T. 13. f. 9. T. 17. f. 32. Gunn. Norv. T. 2, f. 9. IO. I 1. ( Jub L. normoerico )

Ramuli aliguando etiam rugofi, et hine:

Lichen fcaber Huds. Fl. Angl. n. 100.

337. Lichen pubescers - caulefcens folidus teres fcabriufulas ater cæspitofus, ramis sapillaceis confertisfimis adlcendentibus intertextis fibrillois; fcutellis planis concolnribus.

Cornicularia puhescens. Svet. Fjugglaf.

Lichen pubelcens Linndi. Weftr. Act. Sc, Sv. 1799. Laljebl. Huffb.

Lichen lanatus Wulf. ap. Iacqu. Mifc. 2.

Lichen exilis Lightf. Fl. Wiiher (excl. fy: nom. $H u d($.)

Lichen intricatus Ehrh. Cr. exs. Usnea intricata $\mathrm{H}$ offm. Deut. Fl. Icon facqu. Milc. 2. T. 10. t. 5 .

Habitat ad parietes rupium aqua interdum ad. fluente fuifuiarum.

05

$\mathrm{Ob}$. 
Obf. A priori certe distinctus tam ob minutiem of habitum prorfus alienusm, gream ob analyfin longe difcrepantem. - A fuperficie nitida, qua. lem 6. m. Linneus huic tribuit, of ab Ejus apud Dillen. citata figura 9. T. 13, multi fuere decepti, \& vix conjicere potuerust hunc esfe Iichenem, guem jub L. pubescentis nomine in. telleEtum voluat Vir illuftrisfimus.

338. LICHEN velutinus -.. ater pulvinatus ramulofus, ramis confertisimis erectis faftigiatis a. cuminatis.

Cornicularia velutina. Svet. Sammetslaf.

Lichen villofus? Gmel. Syft. Linn.

Bysfus nigra? Pollich P1. Palat.

Collema pannofum? Hoffm. Deut. Fl.

Icon Dillen. Hift. Mufc, T, 1, f, I8? Mich. Gen. T. 90. f. 7?

Habitat ad rupes inter mufcos \& ad fcandula inundata lignea.

Obr. Pulvinato effulus panno fericeo villofo perquam fimilis. In humsido quali gelatino/uss. Receptacita ignota.

Hora. Coninicularias of Phyfcias parum inter pe differre hand egre convincimur, of tranfitus $\mathrm{Li}$ chenusn foliaceorum ad caulescentes mediantibus his fat Juperque demonftratur. Cornicularic vero caules funt magis teretes atque fruticuloo, denfrusque carpitofi, rigidsores of fragiliores quam Phyfcia, fcutellasque gerunt proprie qua/i in. dolis, ratiatas plerumque in infantia of per atatem convexas, torulofas is inequales. Phy $\mathrm{fci}$ a guidem tenuiter intcrdum laciniate \& quasi ra. moja apparent, at foliaceam naturam plus indisitant. 


\section{- 219.- \\ Tribus 27.}

\section{SETARIA. Svet. TRÅdL A F VAR.}

Caules: Lora cartilaginea filiformia teretiulcula nuda glabra, diffufa \& pendula, ramofa, intus tela bombycina farcta.

Receptacula: Scutella planiufculx fesfiles, demum con. vexx, fparfx, margine integrx.

Glomeruli fparfi pulverulenti.

339. Lichen jubatus - caulescens nudus lavis pendulus ramofisfimus albo cinerafcens nigerque, loris filiformibus, axillis compresis ; fcutellis convexis glabris narginatis concoloribus. Setaria jubata. Svet. Mahnlaf.

Lichen jubatus Lirn. Retz. Pr. 2. Hoffb. Lito jebl Weftr. Act. Sc. Svec. 1797. Scop. Lighef. Web. Crantz. Schreb. Spicil Scholl. Weis. Heg. Leers Wild Proir. Ley/s. Roth. Tent. Gunn. Hads. Wi:h. Allsomi. Lumnite. Gmel. Syit. Linn,

Usnea jubata Hoffm. Deut. El. Icon Dillen. Hift. Nufc. T. I 2. f. 7.

Habitat ad ramos arborum precique in Pinetis. Obf. Hujus Iic ditie varietat's, quamvis analytice examinate non evjedenter fatis dircrepant; Tamen non tantum colore niveo, incano, cinereo, fuccos to nigracante, fed etiam forma of habits dis/imiles funt, quare plures of forre diftenitas specio es ex his confersendas fore, fedule et iterate ob. fervationes pollicentur. Inter reliquis eminet:

Usnea ramulofa Hoffin. D: ut. Fl, loris implexis dichotomis cinereo flavefcontibus apice of lateri. buss ramulofo muslitifidis.

Scstellas veras in varietate quadam fu/co nigricante aliquando videre mihi contugit, jed avill ss in hac vire sompres/as reperii. 
340. LICHEN chalybeiformis - caulefcens nudus $I_{x}$ vis decumbens ramofus fufconigricans, loris filiformibus implicato flexuofis, axillis com. prestis; glomerulis fparfis pulverulent is albis. Setaria chalyberformis. Svet. Staltridslaf.

Lichen cbalybeiformis Linn. Retz. Pr. 2. Hoffh. Liljebl. Weifr. Act, Sc. Sv, 1797.

Gunn. Lighif. Schreb. Spicil. Hag. Neck. Scholl: Wrild. Prodr. Roth. Tent. Leers. Gmel. Tubing. Leysf. Pollich. Reich. Fl. Mattufc Craniz, Mull. Mohr. Gmel. Syit. Linn. Lichen jubatus var $\beta$ chalybeiformis $W \cdot c b$. fpic. Wuher.

Usnea chalybeiformis Hoffm. Deut. Fl.

Usnea implexa Hoffom, ibd.

Icon Dallen. Hit . Nufc. T. I3. f. IO. Fl. Dan. T. 262.

Habitat ad rupes, fapes \& truncos arborum. Ohl. Varietatem prioris a laco dependentem puta. runt aliqui, fed ab eo differt, quod minas ra. molus fit, ramis laxioribus magisque clafticis, of guod lemper glonerulos gerat, nunquam aut rarisfime in L. jubato obvios, aliasque per analyfin propriecates oftendat.

34I. Lichen hippotrichodes - caulefcens nudus lavis proftratus capiliaceus fubfimplex fubcompreslus niger, loris ad apicem ramo uno alterove, fcutellis fubglobolis papillofis atris. Setaria kippoirichodes. Svet. Tagellaf.

Lichen hippotrichodes Web. Gmel, Syft. Linn. Lichen pubefcens Leers, herb.

Lichen fotofus Leysf. Fl. hal. Roth. Tent. Bellardi. Gmel. Sylt. I.inn.

Byslus filamentofa Guettard. Obf.

Hypoxylum loculiforum Bull. herb.

Us:ea hippotrichoides Hoffm. Deut, Fl, 
Icon Dillen. Hir. Mufc. T, I3. f, I I. B. Buk. Herb. T. 495. f. I.

Habitat in locis udis \& umbrofis, foliis dejectis conilerarum arborum adharens, mufcis implexus.

Obf. Receptacula ipfe non vidi. An Lichen?

† L، dichotomus - cauleícens giaber ramolus compresio lacinolus pendialus ochroleucus, loris dictoronis alice carillatibus - Usnea dichotona Huffm Deut. Hl. - Dalien. Hift, Muic. T. 13. f. 15?

Obf. An ider cum nofro L. farmentofo? Figura Dilieris fatis quadrat, fed color neuticuarm.

Setaria dicholoma. mont.

f L. flaris - caulefcens rudus lævis fubramofus albus, loris longisfimis complicatis. - Lichen albidus Gmal. Syft. Linn, Schrank FI. Dav, 2. p. 548.

Stearia filaris.

* L. roccella - caulefcens folidus filiformis fubdi. vifus lævis cinereo glaucus; fcutellis hemisphericis fpartis nigris. - Lichen Roccella Linn. Dicks. Alliani. Gmel, Syft, Linn, Sowver6\%. Engl. bot. T. 2 I 1 . Dillen. Hift. Mufc. T. 17.8 .39$.

Obi. Variat ercetus \& pedalis proftratus e Cap. b. fp.

Setaria roccella. rup. marin.

t L. filiquofus - caulefcens folidus viridi cinereus fubfurcatus compresfus demum longitudinaliter fulcatus lisfrilisque; fcutellis lateralibus pedi. 
pedicellatis demum globofis fufco-carneis. Lichen filiquolus Hudf. Fl. Angl. - Gmel. Sylt. Linn. - Dıllen. Hift, Mufc. T, I7. f. 38. Setaria fliguofa. fax. rup.

NotA. Analogiam cum Fungis quam Lichenibus majorem omnino indicant Species sequentes, ad Lichenes aliguando relate, $16:$

Lichen radiciformis $W e b$. Spicil. Swartz. Aa. Upf. V. IV. Retz. Pr. 2. Hag. Wild. Prodr. Roth. Tent. Gmel. Syf. Linn. - Usnea ra. diciformis Scopoli Disf. T. \&. Sichrank. bav. Prim. Sal, atque

Lichen compreslus Ach Wefr. Act. Sc. Sv. 1798. - Facqu. Mifc 1. T. \& Fl. Dan. T. 713. Mich Gen. T. 66. f. 3. - Rhizomorpha fragilis Roth. Uft, Ann. St, 7. Gmel. Sy/t. Linn. - Corallofungus niger Vaill. Par.

41. 8.

$V$ ales etiam hoc de Rhizomorpha capillari Roth Uft. Ann sto 7. T. t f. 3. Gmel. Syft. Linn. of Rhizomorpha Cinchonz Roth. 1. c. T 1. f. 2 Gmel. Syl Linn.

Forte etiam e Lachenum numero exclud adi: Lichen hippotrichotes, L. aidælas, L. pinnatus, L palmarus of I, verricillatus $\mathrm{H}_{\mathrm{umb}}$. ( $\mathrm{Fl}$. Frib cfr. Uft.Ann St. 3 atque ad Genus $\mathrm{H}$ i m a ntize Cl. Perfooni, (kiom. Bot. Magaz. 1.) una cum reliquis jain heic memoratis ipecrebus, petius referende? 


\section{$-223 .-$ \\ Tribus 28.}

US NE A. Svet. HÄR I F V R.

Caules: Lora corrice cruftacea ebducta, filo cenerali ve medullari hyalino eliftico, fubarticulata fcabriafcula teretia, ad befin crasfiura, fenfim attenuata f. brillofa.

Receptacula: Scutella jam farfe fesfiles margine inte. gro, demum convexa fungof $x$ in $x$ quales \& faturatius coloratx, jam planze radiate najores lubtermis naies.

Glomeruli pulverulenti fparf.

342. LICHEN barbatus - cinereo virens pendulus fubdivifus, Joris elongatis fubfilifornibus fibrillofis, fibrillis patenibus; fcutellis minutis fparlis integris fubcarneis demum convexis fuifo nigricantibus.

Usnea barbata. Svet. Skagglaf.

Lichen barbatus. Linn. Ketz. Pr. 2. Hoffo.

Liljebl. Waftr. Ast. Sc, Sv. I798. Lightf. Schreb. Spicil. Weis. Hag. Gunn, Muk? Leers, Roth. Tent. Leys!. Reich, Fl. Allionis。 Scholl. Gmel. Syft. Linn.

Usnea barbata Hoffm. Deut. Fl.

Icon. Sowerby Fngl bot. T, 258. 2. Dillstis Hift, Mufc, T. I2. f. 6.

Habitat ad arborum ramos in fylvis acerofis \& denlis.

343. Lichen comofus - pallidus erectus, loris attenuatis fibrillofis a pice nutantibus; fcutellis Sparfis concoloribus minutis integris demum convexis fufcis torulofis.

Usnea comola. Svet. Tofslaf.

Lichen comofus Ach. Act. Sc, Sv, 1795: 


\section{$-224-$}

Lichen hirti variet. Antorum, Wijir, Act. Sc;

Sv. 1798 .

Icon, Achar. Nov, Act, Ac. Sc. Svac, V. XVI. T. 8. t. $\mathrm{r}$.

Habitat fupra truncos elevaros \& ramos arborum, prefertim Betule albr.

Obl. Canle erecta orasfori fed mulio breviori foutellisque majoribus nec non colore of toto habitse a pracedente differt. - Cum L. hir to of fiorido, receptaculis adhus ignotis, forte frequenter olim, quamvis winus reite commix*us. - Rari!grue Scutelle aliquot in infantia ciliis quabusciam ra. diate occurrunt.

344. Lichen floridus - cinereovirens ereeus, Joris divaricatis brevio:ibus cylindraceis fbrillofis; fcutellis fubterminalibus planis radidis albovirelcentibus.

Usnea florida. Svet. Spollaf.

Lichen floridus Linn, Wefr. Aat Ac, Sc, Sv. 1798. Retz. Pr. 2. Hoffb. Laljebl. Gum. Huds. Allioni, Lightf. Relh. Doerr. Reyg. Schreb. Leers. Crantz. Leysf. Pollich. Reich. Fl. Matiufc. Hig. Wiss. LJeck. Wiid Pr. Kniph. Scholl. Schrank. Bumm. Roth. 'Tent. Gort. Scop. Gronov. Mull. Grsel, Sylit, Linn. Lichen cinchonx Wild. Bot, Mag. Gmel. Syft. Linn.

Usnea florid. Hoffm. Deut. Fl. \& P1. Lich. Icon. Dillen, Hit. Niucc, T, 13, f, 13, Hoffms Pl. Lich. T, 30, f. 2. Morif, T. 7. f 15 . Mich T. 39 f. 5. Tabern. Ic. 808. hit. I195, f. G. Wild. Bot. Mag T: 2 f. 3.

Habitat in frepimentis ligneis vecutis et ad ar. borum cortices.

Obf. Hujus tantum varietas exfortellata of?

Lichen hirtus Linn. Retz. Fr, 2. Hoffo. Eilo 


\section{$-2250^{\circ}-$}

jebl. Hudf. Scop. Lightf. Weis. Pollich. Hag. Neck. Reich. Fl. Mattufc. Gunn. Gort. Letrs. Scholl. Ley T Reyg. Mutl. Mohr. Schveh.

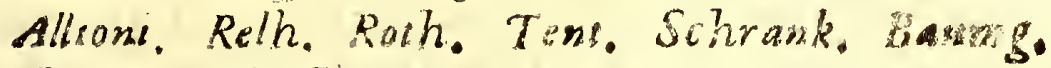
Gmel. Sylt, Linn.

Usnea hirta Hoffm. Deut. Fi. \& P\% Lich

Icon. Dillen.l. .. T. 13, f. I2. Hoffm.l. c. T. 30. f. I. Tabern. Ic 80\%.

L. hirtus Linn. Antor. a Lo flotedn in a tantum differe, quod ille josnior pallistion, glomerulis frequenter sd/per/m, magis ramojus, lonims fibrillofus atque fterilis; hic abstem per atatem fere nigricans, crastior, rigiaior minusgue ramojus fit, of foutellis amplis radiofis inftruatur.

345. Lichen plicatus - albidus pendulus, loris elongatis inplexis fibrillofis; fcutellis fubterminalibus, planis radiatis albo virescen. tibus.

Usnea plicata. Svet. Toflaf.

Lichen plicatus Linn. Retz, Pr. 2. Weftr. Act. Sc. Sv. 1798. Hoffb. Scop. Gronov. Hudd. Lighef. Neck. Web, Hag. Cramtz. Gunn. Mattufc. Allioni, Reyg. Ley/s. Marray Prodr. Wild. Prodr, Roth. Tent. Gmel. Sylt. Linn.

Lichen barbatus var. A plicatus Liljebl. Sv. Fl. Usnea plicata Hoffm. Deut. Fl.

Icon Dilles. Hift. Mufc. T, II, f, I, Tabern. Hift. I I95. f, B, Sowverby Engl, bot. T. $257^{\circ}$ mala.

Habitat ad arborum acerosarum truncos \& ra. mos.

Obf. Hic tantum a T. florido, quantum L. bar. batus a L, comolo difcrepat. 


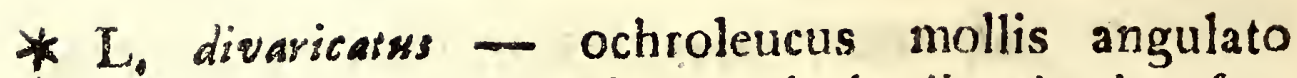
compresfus pendulus, loris divaricatis; fcu. tellis fesfilibus planis rufefcentibus margine nudo integro. - Lichen divaricatus Linn. Retz. Pr. 2. Liljekl. Scop. Allioni. Roth. Tent. Gmel. Sylt. Linn. - Lichen mollis Neck. Meth. - Lichen arenarius? Wcig. Retz. Pr. 2. - Uinea flaccida Hoffm. Deut. Fl. Usnea mollis Baumg. - Dillen. Hift, Mufc. T. I2. f. 5 .

Usnea divaricata, ad arb, acifol, cacum, Jemort.

* L, articulatus - cinereo candicans pendulus fubdivilus, loris fuperne crastioribus articulatis apice capillace is elongatis; fcutellis fubrotundis de. mum rufeficentibus. - Lichen articulatus Budf. Scop. Pollich. Neck. Doerr. Roth. Wither. Sibth. Gmel, Sylt, Linn, - Ufnea articulata Hoffin. Deut. Fl, - Dillen. Hit, Mulc. T. 11. f. 4. - Moris. T. 7. f. I1. Sozverón Engl. bot. T. 258. f. 1. - Mich. Gen. 39. 1.

Usnea articulata. arb.

† L, aurantiacoater - aurantiacus lavis diffufus, loris apice atris; fcutellis fupra atris fubtus aurantiacis. - Lichen aurantiacoater Iacqu. Mifc. 2. T. I I. f, 2. Gmel. Syit. Linn. Usnea auranziacoatra. arb, ad Pol. antarct. 


\section{EMENDANDA Eт SUPPLENDA *).}

Pag.

II. lin. 3, lege: certitudinem

- - 19. - WESTRING,

X. - I. - exftiterint

XI. - 14 \& I5. lege: Lichenum

XII. - 5. lege: concesfum

XIII. - IO. - putavi. - lin, 23, lege: etiam 4. - 8. - Glomeruli

5. - 23 \& 24. deleatur: Dillen. Hift. Mufc. T. 1.f.8.

6. intra lin. 8. \& 9. infere :

Bysfus petræa. Wulf. Beob. Nat, Fr, II, I. lin. 28. deleatur: ve=

8. - penult. lege: Betulx.

9. intra lin. 12 \& 13 infere:

Tubercularia rofea Perfoon Obl. Mycol. 137. poft lin. 13 adjice:

Obf. Ad fungos forte releganda ef hac fpecies.

Hujus etiam funt tubercula illa parva elegan. tisfima coccinea a Leyfero in L. faxatili vi. fa. Cfr. Ehrh. Beytr. 5. 124.

10. intra lin. 17 \& 18 infere:

Lepra botryoides Wigg. Primit.

I I. lin. 32. lege : Lepraria

12. - 4. - cobaltiginea

13. - 8. - conjunxi. - lin: 18. lege: lee.

- 24 - Lichen

15.

- Ad notiliam meam fero admodum perventa eft Cl. SCHiR ADERI Collest. Syftem. Plant. Cryptog. S: Syftemat. Saralunig Krypiogamifcher Gewáchle, 2ite Lieferung, quare ctiam obfervationes in iftis pagellis communicata, neglectx funt, \& Ppecies Lichenum novæ ibidem commemoratx omnino preteritx. Idem quoque valet de Cl. HOFFMANNI Plant. Lichenof. Vol. Ill:tir Faficulo ll:do. Hujus autem Voluminis Fafciculum Primum \& Tertium videre nondum mihi licuit. Itaque ex hife Scriptis ca colligere $8 x$ jam indigitare heic coger, qux cura leftoris luis locis inlerenda iunt. 
15. lin. 9. lege: lavistimus

- 21. adde: Schrad. Krypt, Sanil, n. 173. 18. poit lin. 24. infere:

t L. ellipticus - cruftaceus tenuis albidus; thalamis ellipticis. - Verrucaria Cerali Schrad Krypt. Saml. n. 174. Obf. An idem c L. epidermidis? cujus thalami e. tiam oblongi.

Ferrtecaria elliprica. cort. Cerafi.

21. lin, 26. adte: Dillen. Hift. Mufc. T. 18 f. 2? 2:- 3. deleatur litt. d.

24. intra lin. I \& \& 15. infere:

Licicn foriptus var. A. Hoffm. Enum. Lich. T. 3. f. 2. d.

- lm. 15. arde: Schrad. Krypt. Sanl. n. 168.

- 26. lege: appla.

25. - 19. adde: Schrad. Krypt. Saml. n. 166.

- 22. deleatur litt. $b$.

26. - 12. adde - Lichen fcriptus var. A. Hoffm. Enum. Lich. T. 3. f. 2. b.

- poft lin. 19. infere :

t L. diafporus - crufta fubnulla albida; lirellis minutis difperfis flexuofis finplicibus divifisque.- Opegrapha difperfa Schrad. Krypt.Saml.n.167.Obf.An id.ac L.epipaftus? Opegraph diajpara. cort. Aceris platan. 27. lin. 2022 21. deleatur: Dillen. Hift. Mulc. T. 18. f. 17. A.

30. - $2 r$ lege: agelaus

- 25. agelaa.

33. - 5. lage : tephromelas

37. - 15. - albido

40. - 4 - Q Q

- ult, adde: Schrad. Krypt. Saml. n. I56.

41. - I. lege : cerina

44. - 23. - ferrugineis

44 - 30. adde: Schrad. Krypt. Saml. n. 158. 
45. poft lin. 26. infere:

Patellaria arenaria Koffen. Pl. Lich. T. 58. f. I.

- liv. 20. lege: Icrotllaros dilute rubiginofí, aureo favefcentes vel abidi hunc \&c.

47. - 8. aride: Schrad. Krypt. Saml. n. 159.

- proult. exclude: T. SF. f. \&.

49. - 29. adde: Schrad. Krypi. Saml, n. I55.

5r. intra lin. I \& 2 infere:

Lichen decolorans Schrad. Krypt. Saml. n. 162.

52. lin. 14. lege: cinere - - lin, 24, lege: granu-

53. - 9. - Wither.

-. - 14. adde: Schrad. Krypt. Saml. n. I64.

- - 19 \& 20 excludatur: Dillen. Hift. Mufc, T. 18. f. 11.

56. - 8. lege : concoloribus

58. - 6. adde: Schrad. Krypt. Saml. n. I6I.

60. -m 5. lege: cafis - lin. 12. lege : calcariis

- poft lin. 20. infere:

Lichen calcarius Weis. Crypt. of

Patellaria calcaria Hoffm. Pl.Lich. T. s6. f. 2.que:

61. poft lin. 15 . infere:

Patellaria pantherina Hoffmo. Pl. Lich.

- polt lin. 16. infere:

Patellaria tigrina Hoffm. Pl. Lich.

- lin. 19. adde: Hoffm. Pl. Lich. T. 57, f. I. 2. 63. - 26. lege: forias/is

64. - 26, adde: Schrad. Krypt. Saml, n. I60.

65. - 23. - Schrad. Krypt. Saml. n. 165.

- penult. lege: Jatis

67. polt lin. 14. infere :

Patellaria grileo-atra Hoffm. Pl. Lich. To 60.

f. 2. qua: Verrucaria grifeo-atia Ejusd. Fl.

Germ. forte propria lpecies \& a L. badio diftus.

Ela ; Saltem ex $\mathrm{Cl}$. Hoffmanni figura ita videtur.

68. lin. 6. adde: Schrad. Krypt. Saml. n. 157.

69. - 21. - Schrad. Krypt. Saml. n. I63.

72. - 3. - Patellaria polytropa Hoffm. Pl. Lich.

T. 58. f. 2.

74. 
74. lin. 6. lege: Patellaria

75. - I3. adde : - Piora coronata Hoffm. PI. Lich. T. 56. f. I.

76. - 13. lege: trunc.

77. - II. - Swartzii.

78. - 6. - hemifpharicus

82. - 6. - virefeens

84. - I. adde: Schrad. Krypt. Saml. n. I70.

85. - 6. lege: cularibus

- 8: adde: Schrad. Krypt. Saml. n. 17r.

- - 17. deleatur: lis - lin. 30. lege: crufla

87. - 10. lege: affinia

88. - antepenult, adde: Schrad. Krypt, Saml, n, 132.

90. - 6. lege: Jesfilia

9I. - I4. adde: Schrad. Krypt. Saml, no I 54. Var. $\beta$ Saxatilis ibd.

92. - 3. lege: coralloideos

93. intra lin. 7 \& 8 inlere:

Lichen candelaris Schrad. Saml. n. I so.

97. lin, 20, lege: Hsdlon.

98. - 14. - - decipiente

99. - 29. - chryfolescos

- polt lin. ultimam infere:

+ L. fquamulofus - cruftaceus imbricatus, foliolis appresis angulatis; fcutell mar. ginatis fufco rufefcentibus. - Lichen fqua. mulolus Schrad. Krypt. Saml, n. 153. Obf. Squamæ confertæ iæpius fubimbrica. tæ, crasínflulx, nitidxe, in humido ftatu fordide virides. Scutellæ juniores immerlæx, adultiores elevatæ margine dilco concolore cinctx. An var, I, luridi?

Pforoma fquamulosa. rup. laxa.

100, lin. 6. lege: Maxime - lin. 9. lege: rubris. - 28. tolle : ci in cius;

101. polt lin. 6. infere:

Pfora radiofa Hoffm. Pl. Lich. T. 59. F. I. 


\section{$-231 . \cdots$}

102. poft lin. 12 infere :

Ffora miniata Hoffm. Pl. Lich, T. 60, f. I. - lin, 26. adde: Schrad. Krypt, Saml. n. 152.

104. - 33. lege: 188.

105. - 14. - affociari

105. - antepenult. lege: quidem

107. - 25, lege: cafins

108. polt lin. 3. infere :

Lichen pulchellus Wulf, apud Jacqu. Gmel. Sylt. Linn.

109. lin. 6. lege : cæfio. ultim. adde: Schrad. Krypt. Saml, n, 147.

I 10. - I4. adde: Schrad. Krypt. Saml, n. I48.

I1I. - 27. adde: Lichen tiellaris Schrad. Krypt. Saml. n. 149.

I 2. - 28. lege : cinereo-

I13. - 33. - fubimbri-

$114.24 .-$ T. 24. f. $80^{\circ}$.

117. - 29. adde: Schrad. Krypt. Saml. n. I5r.

I 8. - 29. lege: Kaklaf. - lin. 34, lege: centrituga

$12 \mathrm{I} .-10 .-$ reliquiis

I28. - II. adde: Bernh.Schrad.Krypt.Saml,n,I4I.

- - 12. lege: Collema

- - 26. lege: tenax. - lin. ult. lege : 227.

130. - 5. adde: Schrad. Krypt. Saml. n. 139.

131. - 6. - Schrad. Krypt. Saml. n. 140.

- 7. lege : vefpertilio

135. - 18. adde: - Lichen polyanthes Schrad. Krypt, faml, n. I38.

137. - 20. lege: fcutelliferis - lin, 24 lege: Burges $/ 2 i$

- $\quad 26$ \& 27. lege: muricato

139: - 5. adde: Obf. Varietates hujus funt: Lichen marginalis \& $\mathrm{I}_{4}$ crifpatellus, quemad. modum Cl. Bernhardi obfervasle fertur. Cfr. Schrad. Krypt. Saml. n. 137.

- polt lin. 23. infere: 
Secuidum obfervationes $\mathrm{Cl}$. Bernhardii ap. Schrad. in Krype. Saml. 2. p. u. Lichen criIpus, Collema criliatum Hoffm. ( $L$, cri1tatus?) L. tenax ; L. aphaneus, L. glaucelcens, ejusdem Jpeciei varietases faliztantur, $\dot{\sigma}$ hinc noves condita eft Species, ficlicet: Lichen pulpolus - fronde lobata, 10bis imbricatis dentato crenatis, fcutellis in media planta ubique erumpentibus. Bernh Schrad l. c. - 7 afpo latior faltem videser afe chara-

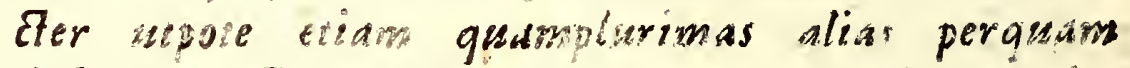

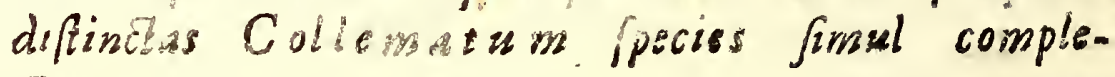
Eens.

140. intra lin, $17 \& 18$ infere:

Verrucaria trapeziformis Schrad. Krypt. Sainl.

$$
\text { n. } 172 .
$$

141. lin. 5. lege: Endocarpon

142. poit lin. 14. adde:

Lichen polyphyllus $W$ ulf. ap. Jacqu, Coll. 144. lin. 27, alde: Schrad. Krypt, faml. n, II 6. - -28. - Hoffm. Pl. Lich. T. 59. f. 2.

145. - I5.' lege: duplici - lin, 2 I, exclude:? 146. - 9. - cribellum

- I 2. adde: Schrad. Krypt. Saml. n. I18.

- - 23. - Schrad. Krypt. Saml. n. I17.

147. - penult. adde: Schrad. Krypt. Saml. n. I 19.

148. - 27. adde: Schrad. Krypt, Saml. n. 120.

157. - 3. lege: Peltigera - lin.20. lege : repando

- - ul. - tomentolus

558. - 30. - f. I. - 3.

159. - 3. adde: - Platisma filix Hoffm. ${ }^{\text {Pl. Lich: }}$

T. 55. f. I. 2 .

- 23. adde: Schrad. Krypt. Saml, n. I22.

I6r. - 20. lege: amplisfimis,

I62. - 2I. adde: Schrad. Krypt. Saml. n. I2I. 564. - 3. lege: Lichen refupinatus $V$ ar. $A$. lap. ponicus Liljebl. Sv. Fl. 
165. lin، 6. adde polt L, flaccidum: $(n, 232$.

- - 19. lege: Icon.

166. - 26. adde: - Lichen chlorophyllus Humb. - Peltigera chiorophylla, margine adicen. denti.crifpo, fuperne læviulcula inferne venofa pallefcenti-albida, fcutellis rutefcentibus, Wilden. Phyt. Fafc. 2. Schrad. Krypt. Saml. n. 123. - Obf. Specimina a $\mathrm{Cl}$. Dickjoni mista ego tantum vidi, qua characterem fubminiftrarunt, \& Ejus fide Wulfenii lynonymon adjeci. Sin vero Species Dickfonio ab ifto Wildenowii \& ulfenii difincta lit judicent autoptz.

167. - I5. lege: 276. - ibd. lege: fub-

168. - antepen. adde: Schrad. Krypt. Saml. n. 145. 169. - antepen, adde: Schrad. Krypt. Saml. B. I 46.

174. - 5. adde: Schrad. Krypt. Saml. n. I44.

- - 23. loco fuicis lege: badiis

175. - 14. lege: Phy/cia - lin. 30. lege: Quercus

176. - 6. adde: Schrad. Krypt. Sami, n. 143.

179. - I5. lege: varietatum

- 32. \& reqq. lege:

LICHEN vulpinzs - cartilagineus virefcenticitrinus erectus compreslus in $x$ quali angulofus ramofistimus attenuatus fubfaftigiaius,

- ult. lege: vulpina.

180. - 13 \& 14. lege : exterior quafi cruftofa, fuper. ficie interdum pulverulenta,

I8I. - 8. loco lept, lege: merid. \& Inf, Mar. auftr. - 30. lege: arbor.

182. - 7. - lineari - lanceolatus

- 29. - diffufus - lin. 27. lege: attenuatus;

183. - 4. - diffufus loco proftratus

184. - 3. - cartilagineus - lin. 30. lege. foliola

- - 30. - cario - virentibus;

- - ult. lege: Scyphophorss

$1850-\frac{-}{P_{5}}-$ fupergine

188. 


\section{$-234 \cdot-$}

188. lin. 20. lege: Hujus

- -30 - nereo-pallidis cylindraceis

191. - 10. - gracilis - lin, 28, lege: marginegre

- 20. - deleatur: Lichen polyceras ibd.

194. $\rightarrow$ 7. deleatur: in

195. - 8. lege: Hoffino

- poit lin. 27. iniere:

Lichen iquamolus irregularis Schrad. Krypto Sanl. n 135 .

197. lin. 4. lege: qua - lin. 7. lege: specifica 198. - 25. adde: Schrad. Krypt Saml. n. I33.

199. - 8. - Sthrad. Krypt. Saml. n. 194.

II. - At Jecurdum Cl. Schrad. Krypr. Samb.

l. c. hujus fpecimina guafi varietates $\mathrm{L}$. cocci. feri potius cenfenda.

201. lin, 21. adde: Schrad. Krypt. Saml. n. I31. 202. - 2I. lege : fylvarum.

203. - 4 adde: Schrad. Krypt. Saml. n. I29. - poft lim. 15 infere:

Lichen racemofus Schrad. Krypt, Saml, no 130. qui :

205 lin. 29. adde: Schrad. Krypt. Saml. n. I28. $210-26$. - Schrad. Krypt. Saml. n. 169. $215-7 .-$ Schrad Krypt. Saml, n. I26.

- 27. lege: neque

215. - 3. - terminalibus

217. - 13. adde: Schrad. Krypt. Saml. n, 12\%. 219. - 19. - Schrad. Krypt. Saml, n. 25. 220 - penult. lege: loculiferum 225. - 26. adde : Schrad. Krypt. Saml, n. I24.

Recenfentur in hoc Opufculo fancita Lichenum Species: 529 ; quas inter Nova: IOI; Svecice indigine 245: xira patriam adhuc tantummodo reperte: 184; \& praterea his omnibus nominatims (ubjuncta Varieta. tes: 202 - Summa Specierum \& Varietatum diftincta. rum: 73 !; Aique Synonymorum collectorum \& ad fpeci. es certiores legitimasque redactorum $; \mathbf{1 3 7 8}$. 


\section{INDEX NOMINUM.}

Synonyma curfivis litteris reddita funt. Numerus paginam indicat. Afterif́ci duo ( $\left.{ }_{*}\right)$ l. tres ( $\left(w_{*}\right)$ numero adjêii indigitant quod bis vel ter idem nomen fub diverfis fpeciebus in eadem pagina occurrat.

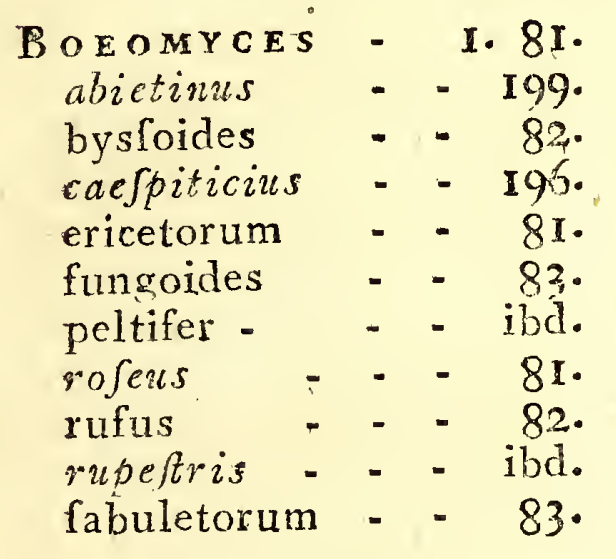

Bysus.

\begin{tabular}{|c|c|}
\hline arraginos $s a$ & 10. \\
\hline antiquitatis & - - \\
\hline aurea & II. \\
\hline botryoides & - \\
\hline candelaris & $-=$ \\
\hline cobaltiginea & 12. \\
\hline cryptäum & \%. \\
\hline fllamento $\int a$ & $=220$. \\
\hline incana & $7 \cdot$ \\
\hline jolithus & Ir. \\
\hline lactea & $7 \cdot$ \\
\hline nigra & 218. \\
\hline petraea & $-227 \cdot 6 \cdot$ \\
\hline rofea - & \\
\hline faxatilis & \\
\hline A I ICIUM & \\
\hline $\begin{array}{l}\text { aciculare } \\
\text { cantherellum }\end{array}$ & $\begin{array}{l}=86 . \\
=\quad 85 .\end{array}$ \\
\hline capitatum & $-\quad-86$. \\
\hline clavellus & $-\quad 83$. \\
\hline $\begin{array}{l}\text { corynellum } \\
\text { helopherum }\end{array}$ & 85. \\
\hline hyperellum & $=85$. \\
\hline
\end{tabular}

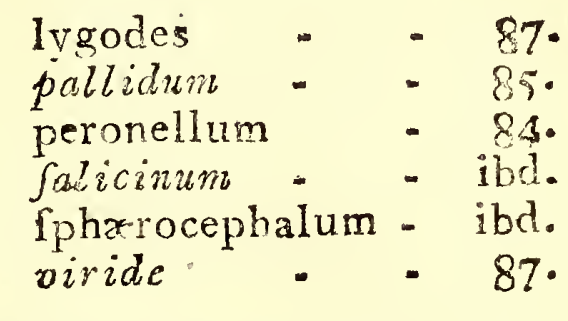

CIADONIA: $3.20 \mathrm{~T}$. aggregata - - 206 . attenuata - - 202. biuncialis. - ibd. botrytes - - 199. ceranoides - $20 \mathrm{r}$. coccinea . - 189. cornucopioides - 195. cornuta - - $\mathbf{1 9 3 .}$ coronata - - 189. deformis - $-\mathrm{ibd}$. denticollis. - 195. digitata - - 189 . dilatata - = I92. elongata - - I9h. exilis - $\quad \mathbf{2} 86$. extensa - - 183. fibula - - ro4. fimbriatc - - I87. foliacea - - 184. fungiformis - 82 . furcata - - 204. furcato - - ibd. furcato fubulata - ibd. furcellata - - ibd. furcellatoradiata 191. $f_{\text {usca a }}$ - I98. 199. gracilis - . I9I. hybrida * - ibd. incan $\Leftrightarrow-186$. 


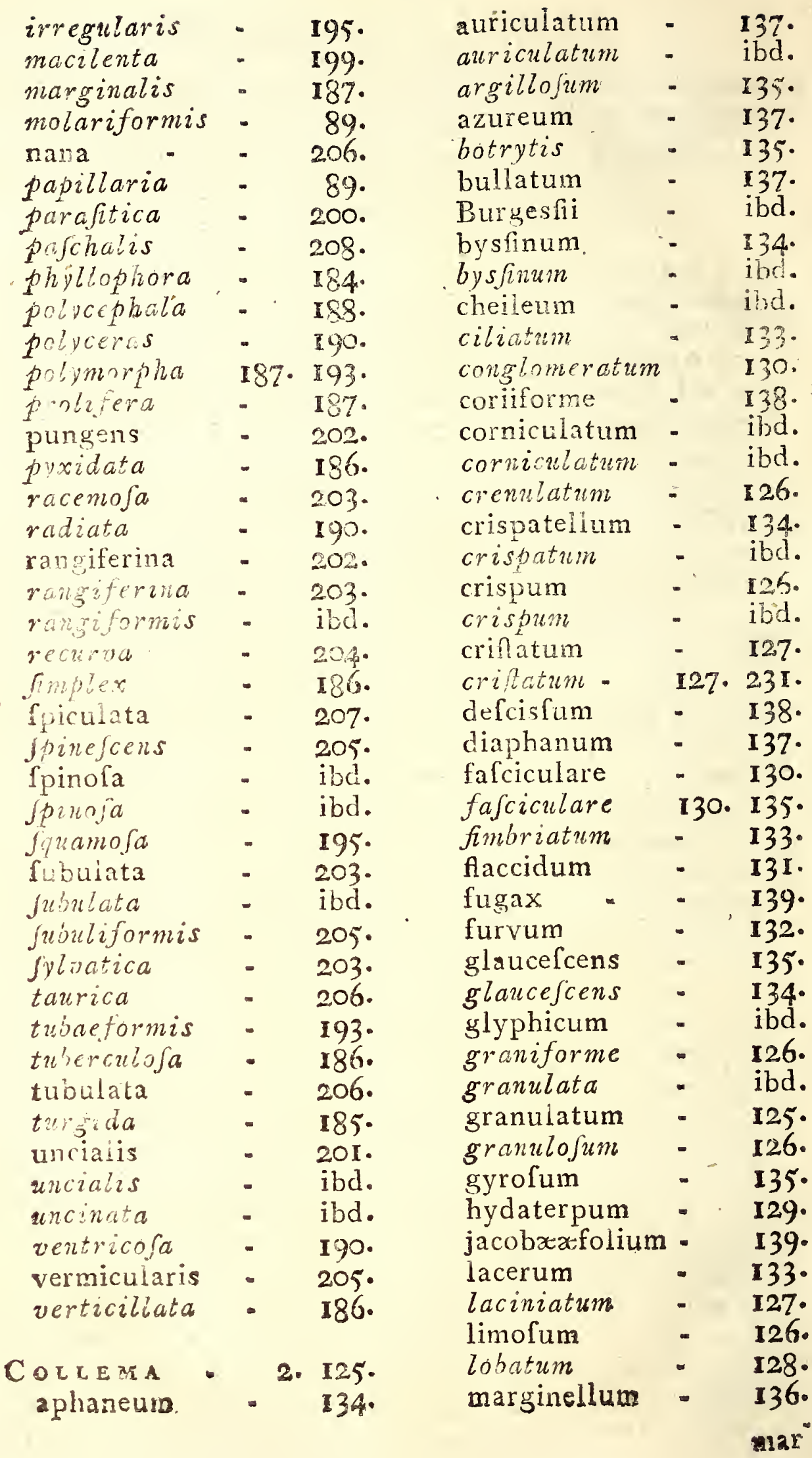




\begin{tabular}{|c|c|c|c|c|c|}
\hline marginale & - & $\times 27^{\circ}$ & lanata & - & $2 \pi 6$ \\
\hline marginale & 134. & 136. & muricata & 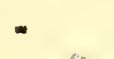 & 21 \\
\hline meitrum & & 130. & muficicola & & \\
\hline m;riococcum & & 127 & ochroleuca & - & 215 \\
\hline nisrefcons & & $13 \pi$ & ntella & $\cdot$ & \\
\hline nignn - & . & 92. & pubefcens & - & 217. \\
\hline objernnmi- & & $13 \hat{4}$ & triftis & * & \\
\hline opurtiobes & & 136. & trifiss- & - & \\
\hline palmatum & & 136. & velutina & - & \\
\hline pannonam & & 218. & & & \\
\hline plicatie & & 129 & IMEOLUS & & \\
\hline plicatrim & - & 136. & furfuracea & - & \\
\hline polycarpum & - & 135 & fepulchralis & - & \\
\hline polycarpon & a & $\mathrm{ibd}$ & & & \\
\hline prafinum & - & 138 & ENDOCARPON- & 2. & \\
\hline hrom & - & $i b d$ & cinercum & - & \\
\hline pulviratum & - & $133^{\circ}$ & olicatum & - & $I 68$ \\
\hline ramulojum & - & $2 \pi 6$ & ocum & - & \\
\hline rivulare & - & 132 & Hedwigii & - & \\
\hline sinum & - & ibd & lac & - & \\
\hline fcotinlim & & 128 & phyllúm & - & \\
\hline fedifolium & - & 13 & tum & - & \\
\hline finuatum & - & 128. & murinum & & 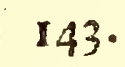 \\
\hline finuatum & 128 & $133^{\circ}$ & $p u f i l l u m$ & - & 14 \\
\hline fubtile - & - & 136 & llatum & - & 14 \\
\hline fubtile - & - & ibd & nbergii & - & 14 \\
\hline fymphoreum & - & 13 & dulum & - & \\
\hline fynalizum & - & ibd. & Weberi & - & 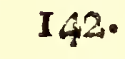 \\
\hline tenax & - & $128^{\circ}$ & & & \\
\hline tenuisfimum & - & 139. & ODIUM - & $3 \cdot$ & $\cdot I$ \\
\hline tenuisfimum & - & 139. & tes & - & \\
\hline tomentofum & - & 132. & ofum & - & 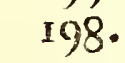 \\
\hline tremelloides & - & & delicatum & - & \\
\hline $\begin{array}{l}\text { tunxforme } \\
\text { ve pertilio }\end{array}$ & - & 132. & fymphycarpum & & \\
\hline
\end{tabular}

Corallotdes.

$\begin{array}{lll}\text { aculeatum } & - & 213 . \\ \text { fragile } & - & 211 . \\ \text { globiferum } & - & 210 . \\ \text { paschale } & - & 208 .\end{array}$

CORNICULARIA.4. 2 I2. aculeata - 214. bicolor - 215 .

HELVELIA Sarcoides - 139.

HYPOXYL ON loculiferutn 220.

IMBR I CARIA - 2. IOZ. aipolia - Ir2. aleurites $\quad-\quad \mathbf{I} \eta$. 2moli- 


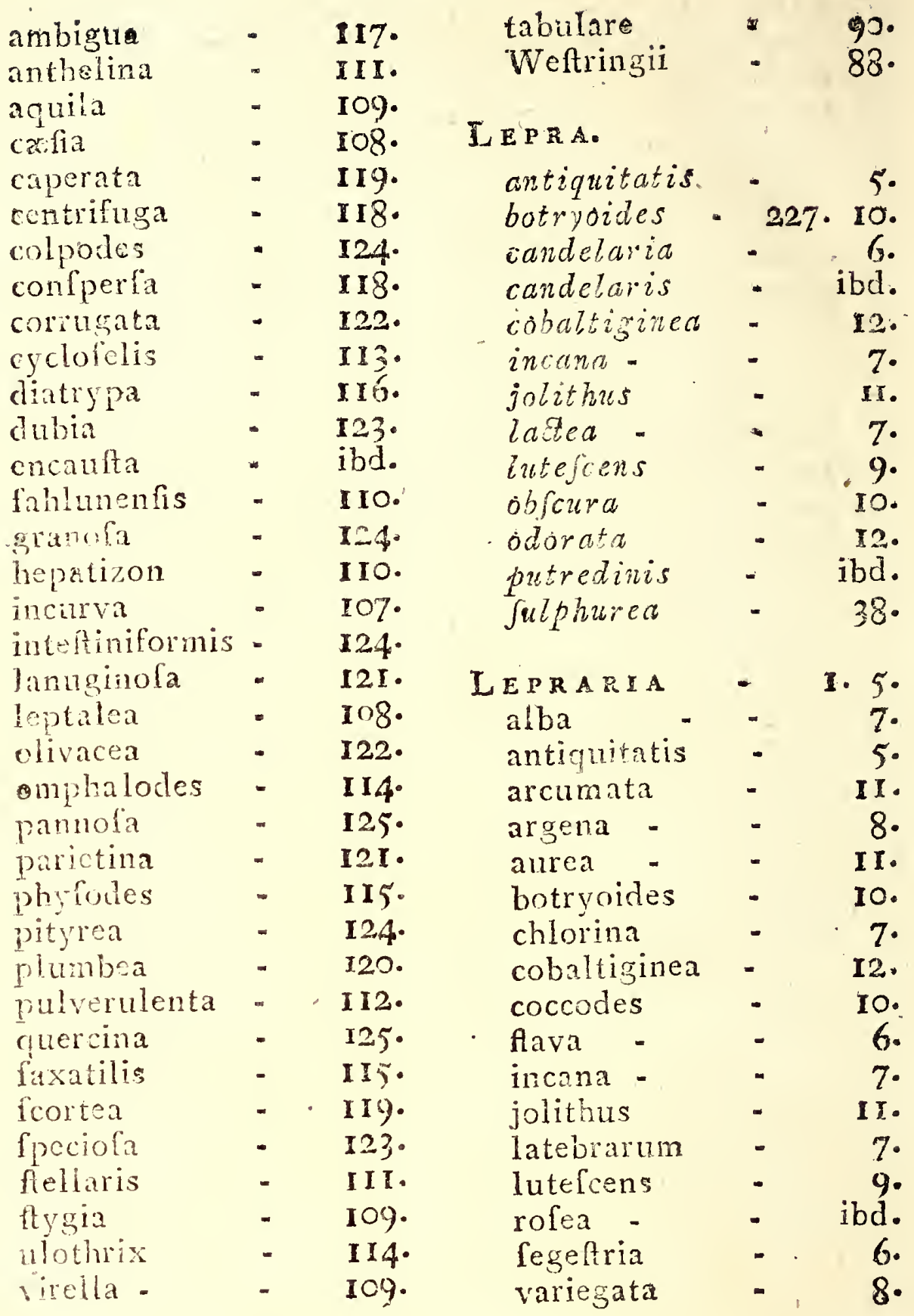

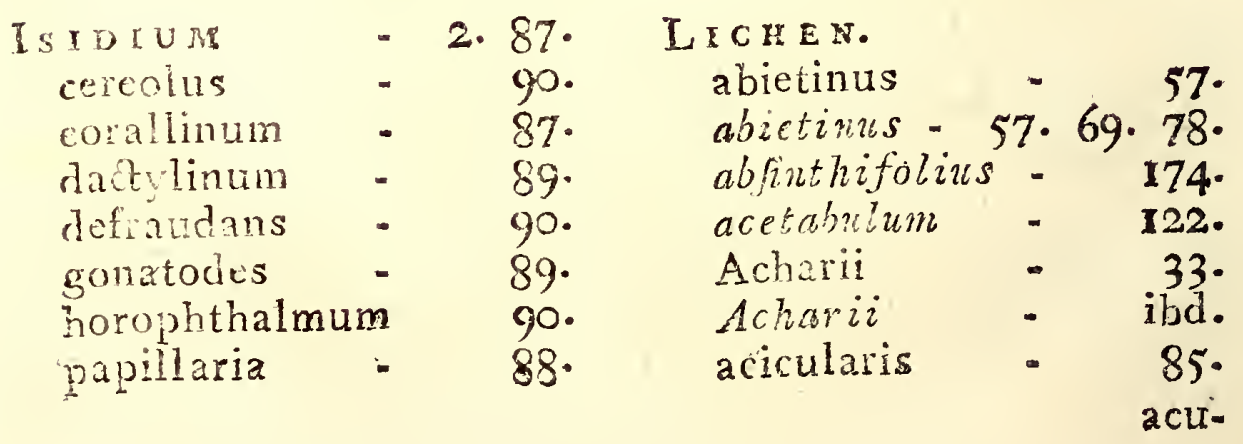




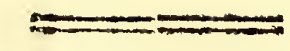

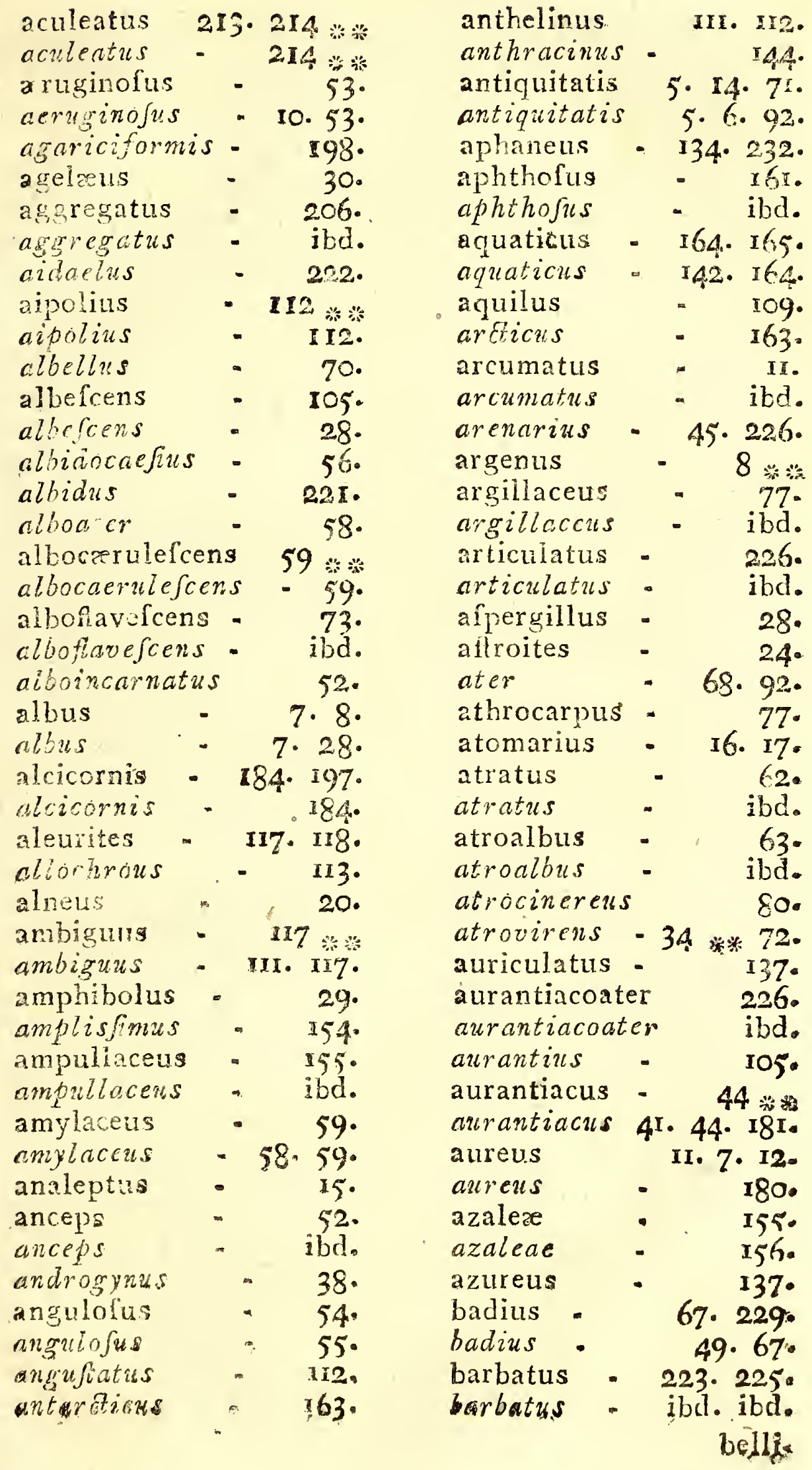




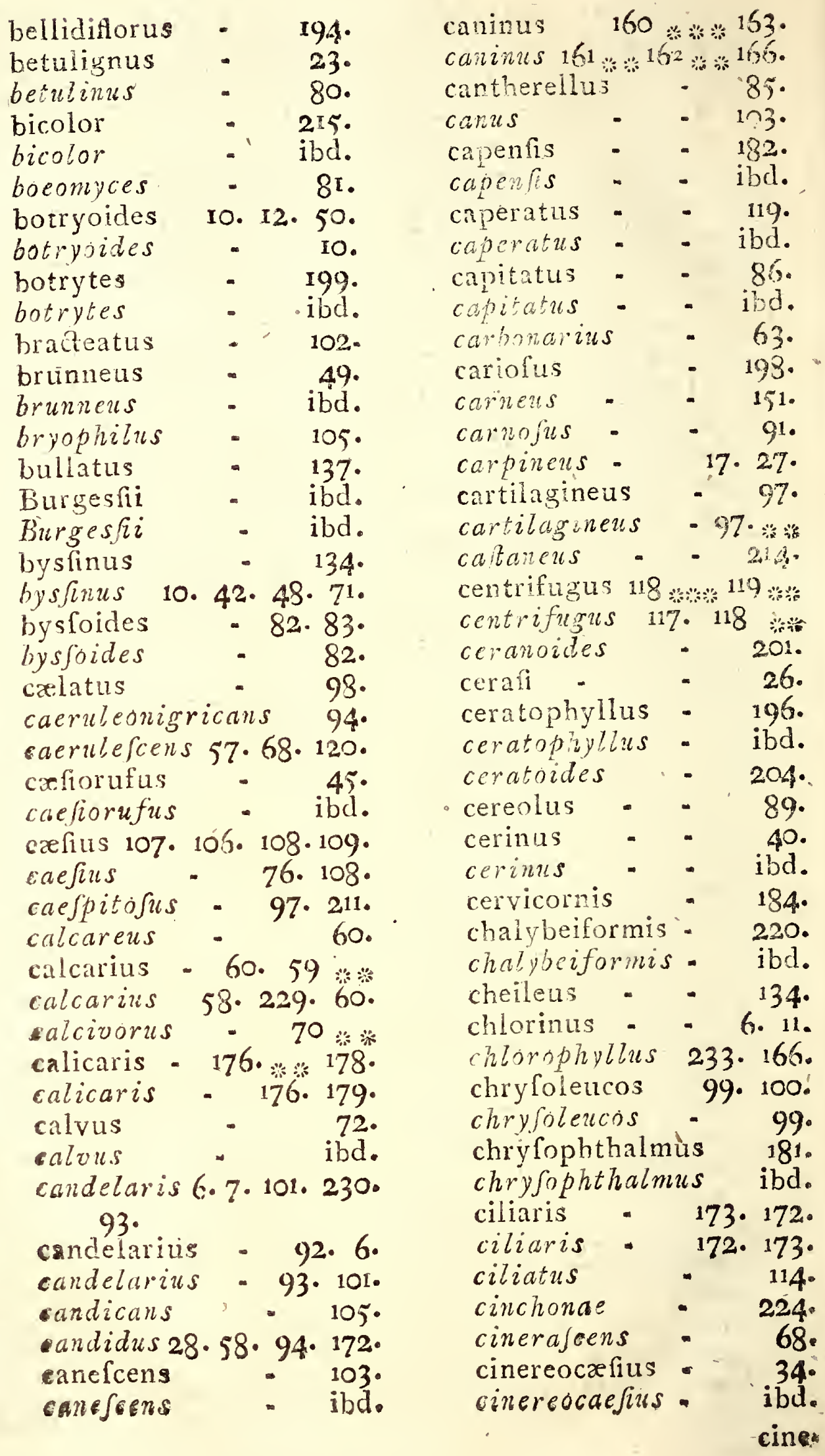




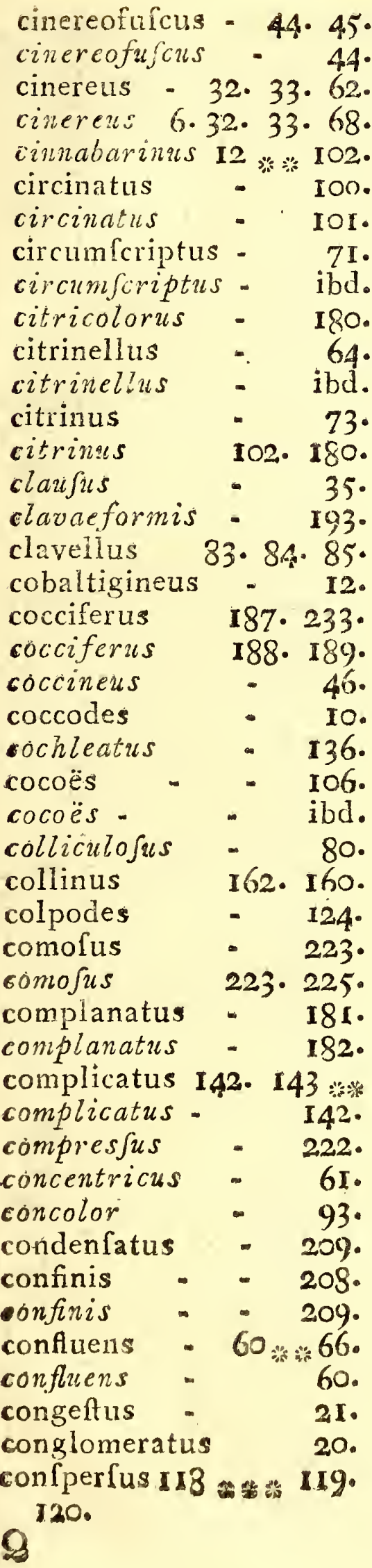

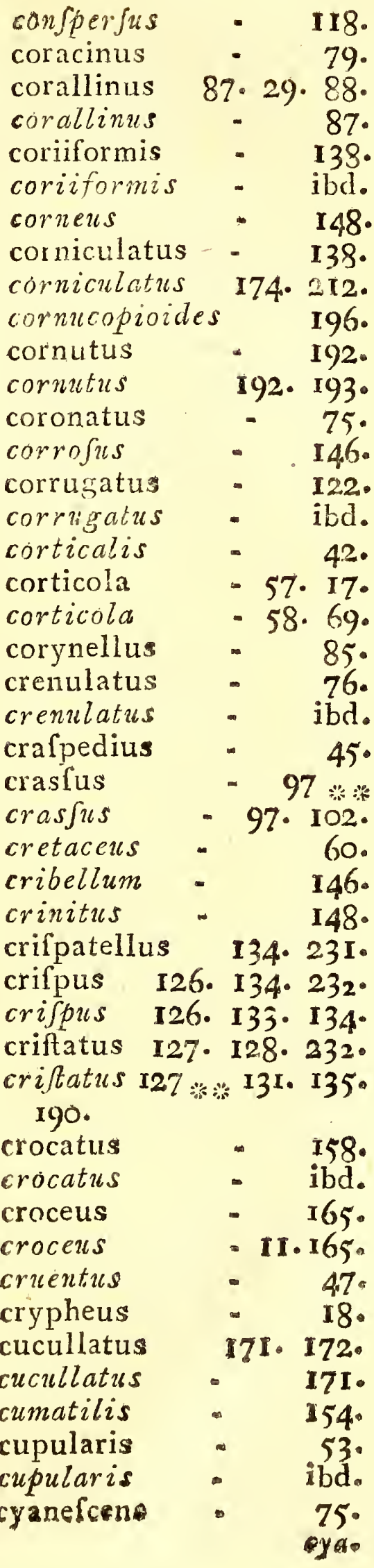




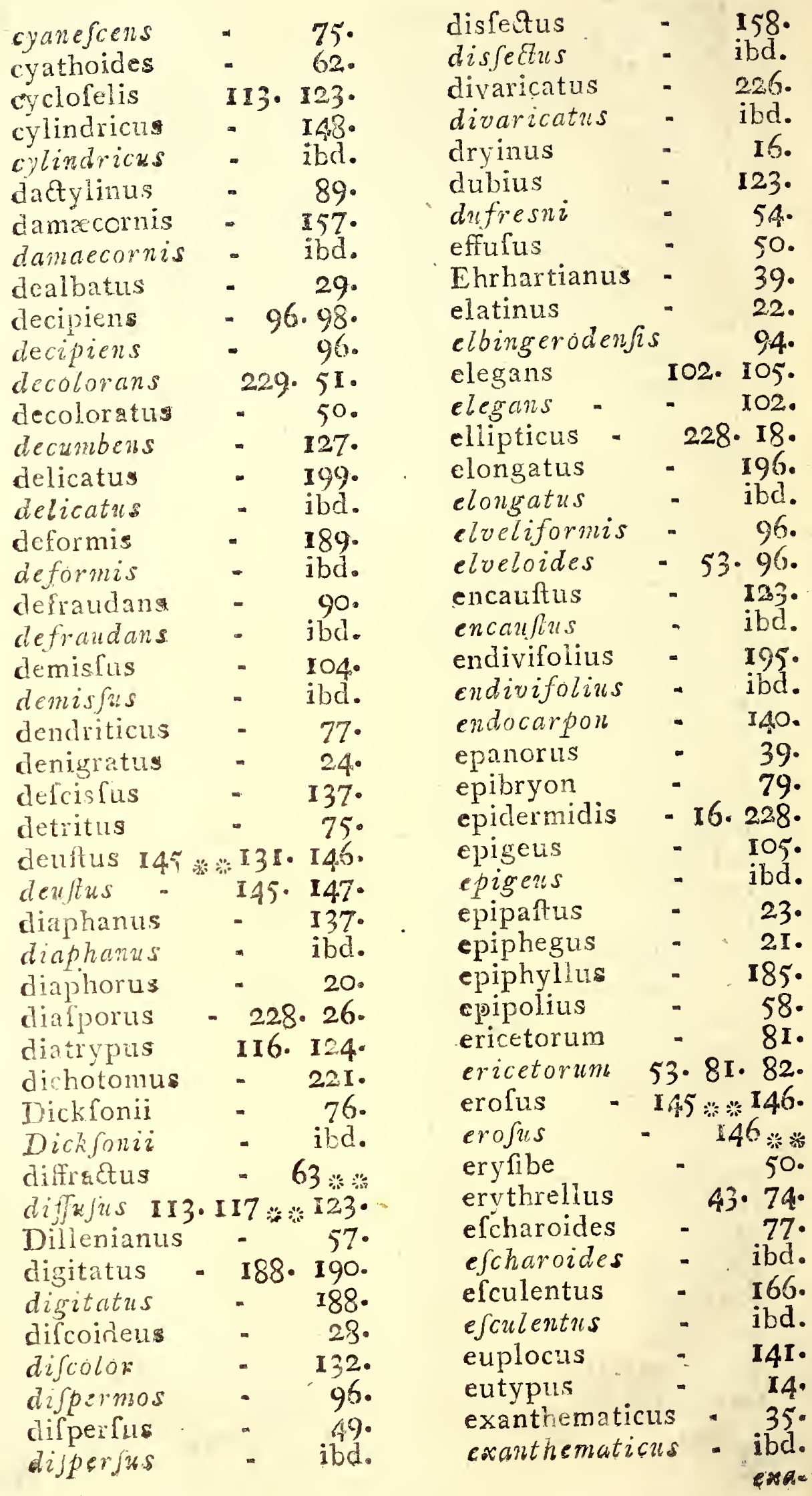




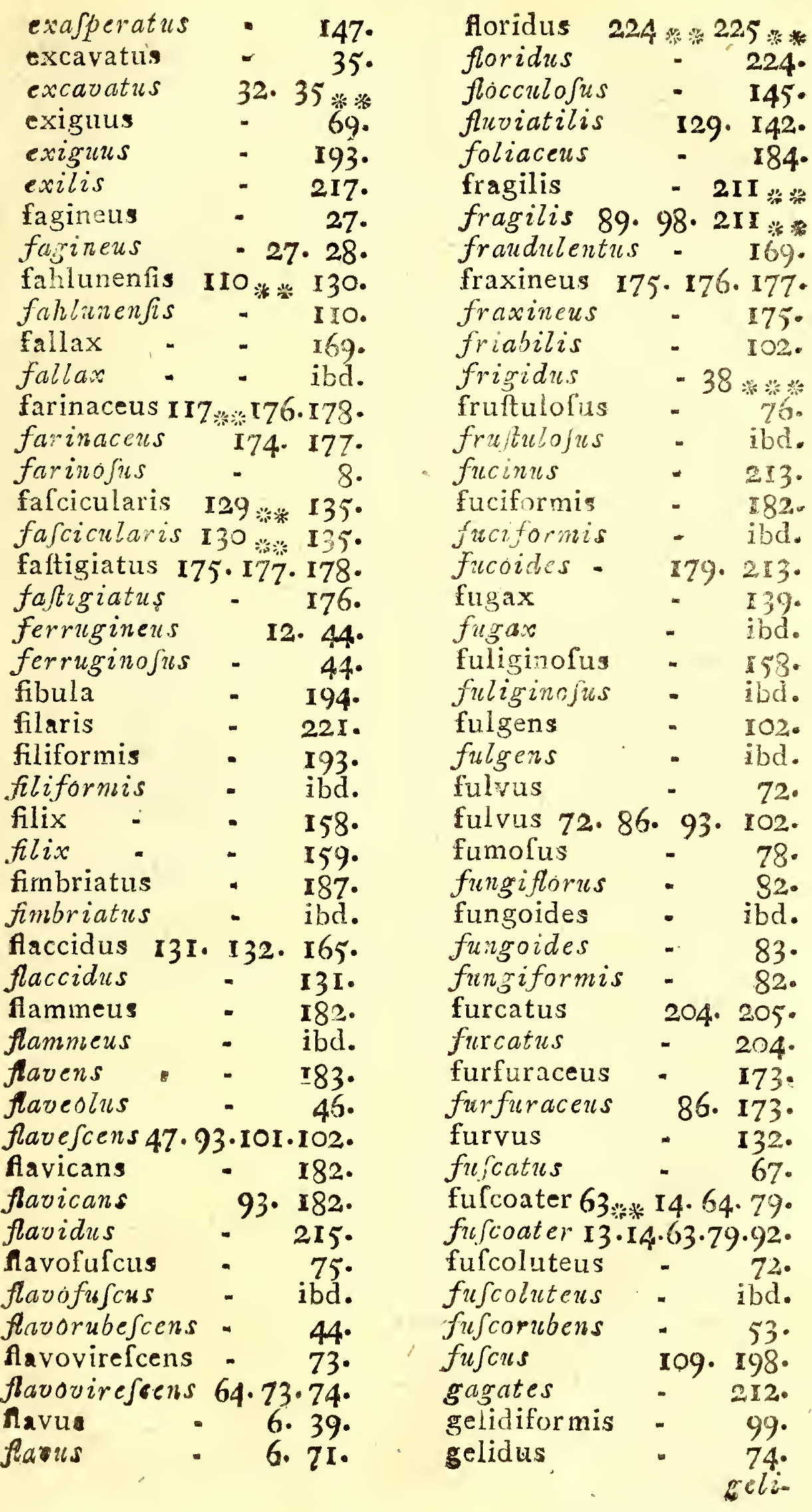




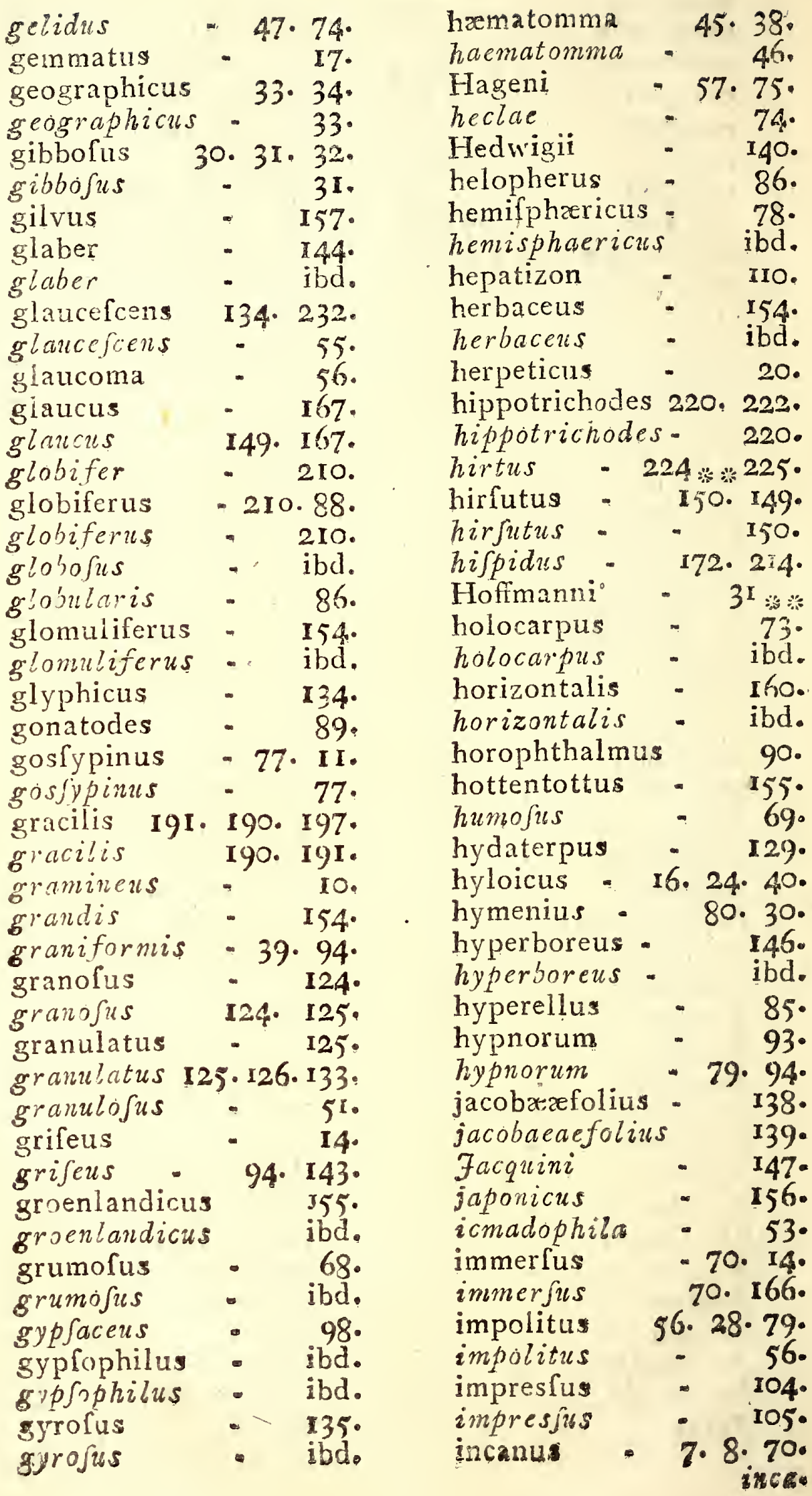




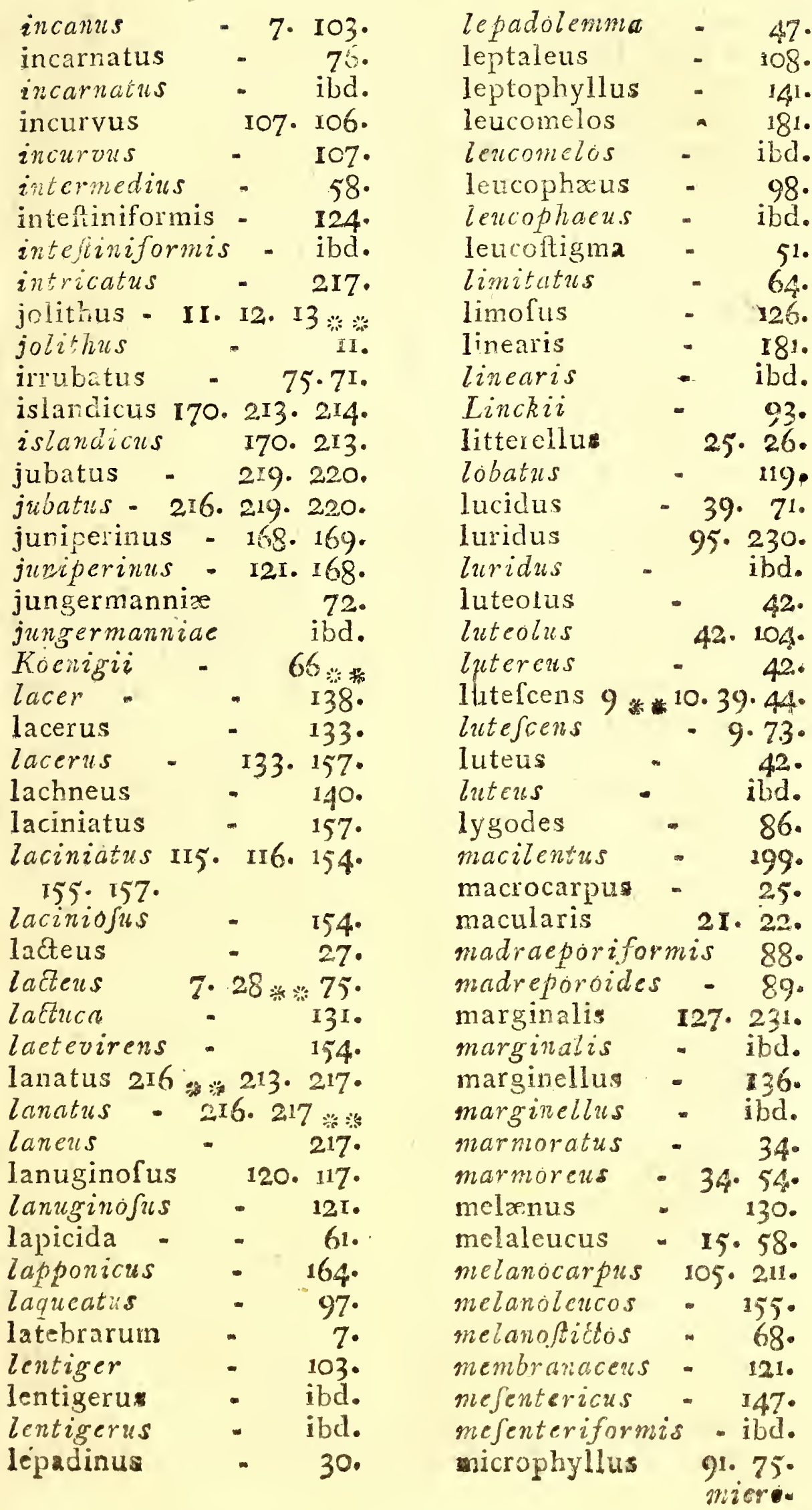




\begin{tabular}{|c|c|c|}
\hline nicrophyllus & $s$ & ochrochlorus - \\
\hline miniatus - & $141 \cdot 142$. & chlorus \\
\hline $\begin{array}{l}\text { iniatus } 10 \text { ? } \\
\text { inutisfimus }\end{array}$ & 02. $141 \cdot 142 \cdot$ & $\begin{array}{l}\text { chroides } \\
\text { chroleucus }\end{array}$ \\
\hline $\begin{array}{l}\text { sinmus } \\
\text { sflmus }\end{array}$ & 40. 76. & ochroleucus.96.104.171.215. \\
\hline ellus & 62 & oculatus \\
\hline $\begin{array}{l}\text { mollis } \\
\text { monocarpus }\end{array}$ & 226 & tellus $213 \cdot 214 \cdot$ 婉 \\
\hline carpus & I96. & odoratus \\
\hline tifidus & 70 & $34 \ldots 66 \approx$ \\
\hline illorus & $\begin{array}{l}-\quad 49 \\
-\quad 49\end{array}$ & $\begin{aligned} 34 * * 00 \\
121 \cdot x 10 \cdot x 23\end{aligned}$ \\
\hline $\begin{array}{l}\text { ultipunctat } \\
\text { ultipunctus. }\end{array}$ & tus & olivaceus \\
\hline $\begin{array}{l}\text { ipunctus. } \\
\text { alis. }\end{array}$ & 123 & $114 \cdot I 15$ \\
\hline $\begin{array}{l}\text { alis } \\
\text { catus }\end{array}$ & -104. & $113 \cdot 114$ \\
\hline 3 & $214 * 2235$ & $\begin{array}{r}138.133 \\
-\quad 138\end{array}$ \\
\hline $\mathrm{m}$ & $-\quad 101.102$ & $\begin{array}{l}\text { opuntioudes } \\
\text { orbicularis }\end{array}$ \\
\hline & - 101. I26 & orbiculatus \\
\hline & $\begin{array}{l}215 \\
216\end{array}$ & 137. \\
\hline & $=69^{21}$ & $\begin{array}{l}38.90 \\
=\quad 36.37 .\end{array}$ \\
\hline 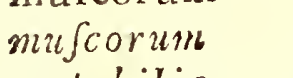 & $\begin{array}{l}=69 \cdot 8 \cdot \\
=69 \cdot 105 .\end{array}$ & $\begin{array}{l}\text { pallefcens } \\
\text { pallescens } 36 \cdot 37 \cdot 40 \cdot 55^{* *}\end{array}$ \\
\hline ilis & - $\quad 154$ & $\begin{array}{l}\text { pallejcens } 36.37 \cdot 40.55 \\
\text { I17. }\end{array}$ \\
\hline rous & 132 & pallidus. 54.55**215 \\
\hline 40 & $\begin{array}{l}127 . \\
206 .\end{array}$ & $\begin{array}{lll}\text { pallidoniger } & - & 8 \\
\text { pallidoniger } & - & \text { ibc }\end{array}$ \\
\hline ger & 92.6 & $\begin{array}{l}\text { pallidoniger } \\
\text { palmatus }\end{array}$ \\
\hline ger & 9 & $\begin{array}{l}\text { paimatus } \\
\text { palmatus }\end{array}$ \\
\hline grefcens & 13 & pannofus \\
\hline cens & 13 & pannofus \\
\hline$n s$ & $\begin{array}{r}68 \\
71\end{array}$ & pantospermus \\
\hline 21 & & papillaria \\
\hline us & $-\quad 18$ & $\begin{array}{l}\text { pafillaria } \\
\text { papy?aceus }\end{array}$ \\
\hline & ${ }^{17 i}$ & parafemus $64 \cdot 65 \cdot 66.6$ \\
\hline $\begin{array}{l}\text { alis - } \\
\text { thus - }\end{array}$ & $168 \cdot 17$ & us \\
\hline othus - & $\begin{array}{r}19 \cdot 17 \cdot 58 \\
212 \cdot 212\end{array}$ & loxus \\
\hline eratus & $\begin{array}{r}212 \cdot 21 \\
-7\end{array}$ & $\begin{array}{l}\text { parallelus } \\
\text { parafiticus }\end{array}$ \\
\hline ratus & - ibc & $\begin{array}{l}\text { parajulcus } \\
\text { parechus }\end{array}$ \\
\hline us & $-\quad 2$ & - $\quad 36.37$ \\
\hline & 109. $114 \cdot$ & parellus \\
\hline tus & $\begin{array}{l}-209 \\
-\quad \text { ibd }\end{array}$ & $\begin{array}{l}\text { parietinus } \\
\text { parietinus }\end{array}$ \\
\hline & -6 & -164 \\
\hline & 32. & $\begin{array}{l}208.200 \\
\text { paseho }\end{array}$ \\
\hline
\end{tabular}




\begin{tabular}{|c|c|c|}
\hline paschalis & 209 & polyceras \\
\hline patellatus & $r 22$. & polydactylus \\
\hline pellitus & $140 * *$ & polydactylus \\
\hline pellitus & 150. & polygonius \\
\hline peltifer & $83 \cdot$ & polygonius \\
\hline peltifer & ibd. & polymorphus \\
\hline peltiphyllos & 96. & tolymorphus \\
\hline $\begin{array}{l}\text { perforatus } 1 \\
\text { perforatus }\end{array}$ & $\begin{array}{c}155 \cdot 153 \cdot 165 . \\
=\quad 155 .\end{array}$ & $\begin{array}{l}\text { x87** I93. } \\
\text { pólyphyllus 144.232.I4 }\end{array}$ \\
\hline pericleus & -78.69. & polyrrhizos 148.140 \\
\hline eriatus & 153. & $150 * *$ \\
\hline $2 u s$ & ibd. & polyschides \\
\hline llus & 84 . & polytropus \\
\hline Perfoonii & 19. & polytropus \\
\hline pertufus - & $17 \cdot 30 * * 88$. & populinus \\
\hline pertufus $x$. & - 32.80 .226$. & populneus \\
\hline petracus & GI. & porphyrias \\
\hline petrofus & r3. & pracindus \\
\hline $\begin{array}{l}\text { pezizoides } \\
\text { phyllophorus }\end{array}$ & $s=\quad 49.96$. & $\begin{array}{l}\text { probolcideus } 147 \cdot 14 \\
\text { probofcideus } 147.148 .15\end{array}$ \\
\hline phyfodes - II & $115 \cdot 1 \times 7 \cdot 124^{\circ}$ & proteiformis \\
\hline physodes & $=I I \sigma * \star$ & pruinatus \\
\hline picinus & I4. & pruinofus \\
\hline pictus & 106. & pruinofus \\
\hline pictus & ibd. & prunaftri \\
\hline pilularis & & prumaltri = \\
\hline pilularis & ibd. & pseudocorallinus .88 \\
\hline pinaftri & 168. & pfora \\
\hline Ar $i$ & ibd. & pubefcen \\
\hline pinicola & $=66.69$. & pubescens \\
\hline pinnatus & 222. & 220. \\
\hline pityreus & 124 & $\begin{array}{l}\text { pulchellus } \\
\text { pulcher }\end{array}$ \\
\hline pis & $\begin{array}{l}-\quad 18 . \\
=\quad 195 .\end{array}$ & $\begin{array}{l}\text { pulcher } \\
\text { pulcher }\end{array}$ \\
\hline plicatilis & $129 * 130$. & pulmonarius \\
\hline plicatilis & 129. & pulmonarius \\
\hline plicatus & 225. & pullus 100.122. 144.14 \\
\hline plicatus & ibd. & pulposus \\
\hline plumbeus & $-\quad x 20$. & pulverulentus - 112 * \\
\hline plambeus & -120.152. & pulverulentus ios.112.134 \\
\hline ris & $-\quad 163$. & punctatus. \\
\hline $\begin{array}{l}\text { pollinarius } \\
\text { pollinarins }\end{array}$ & $\begin{array}{l}178 . \\
\text { ibd. }\end{array}$ & punctiformis \\
\hline $\begin{array}{l}\text { pollinarius } \\
\text { polyanthes }\end{array}$ & $\begin{array}{l}-\quad \text { ibd. } \\
-23 I_{0} 135 .\end{array}$ & $\begin{array}{l}\text { panctiformis } \\
\text { pungens }\end{array}$ \\
\hline pus & $=\quad 135$. & puftulatus \\
\hline polycarpus & $23 \cdot$ & puftulatus \\
\hline
\end{tabular}




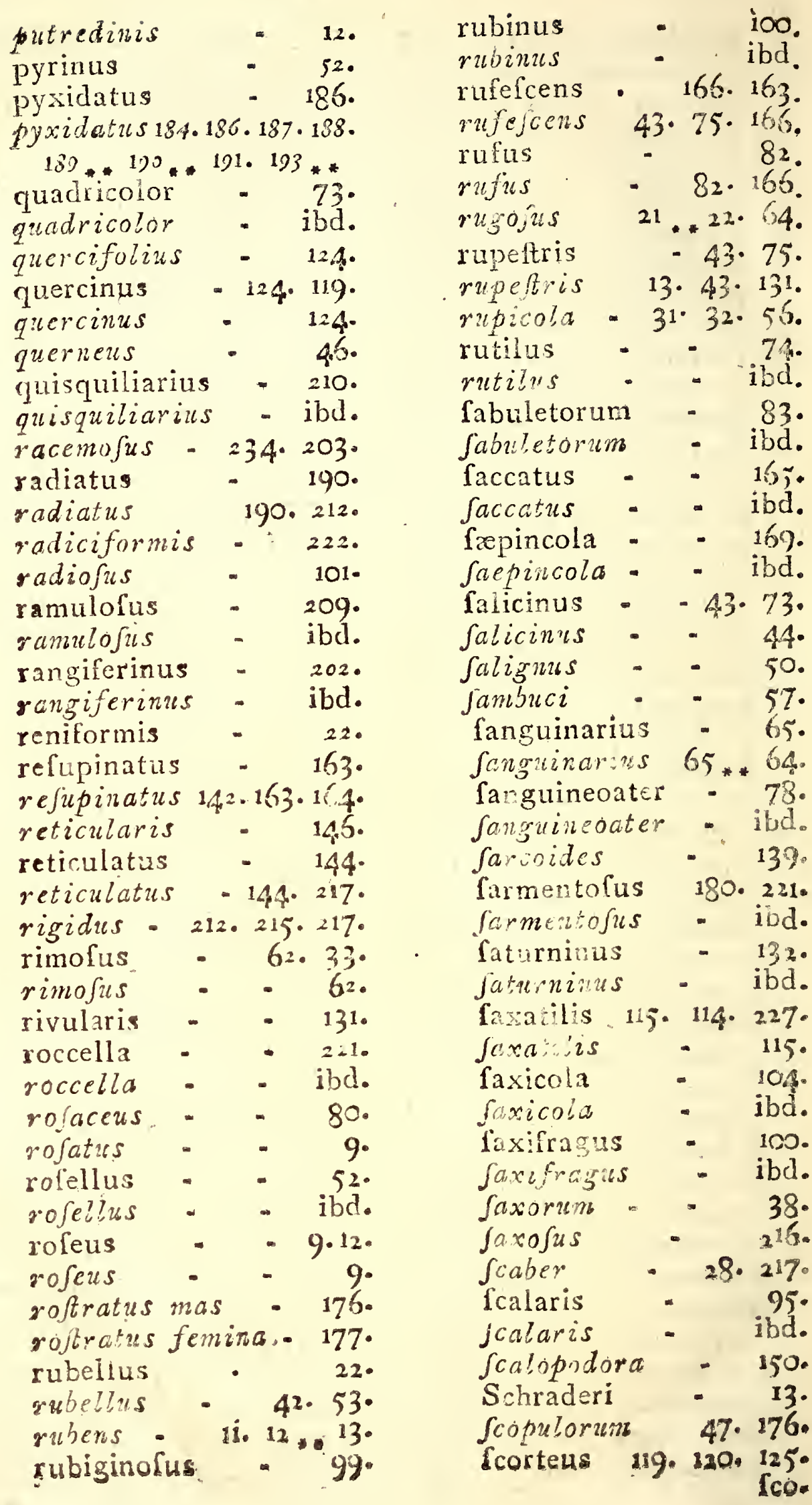




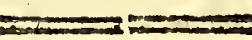

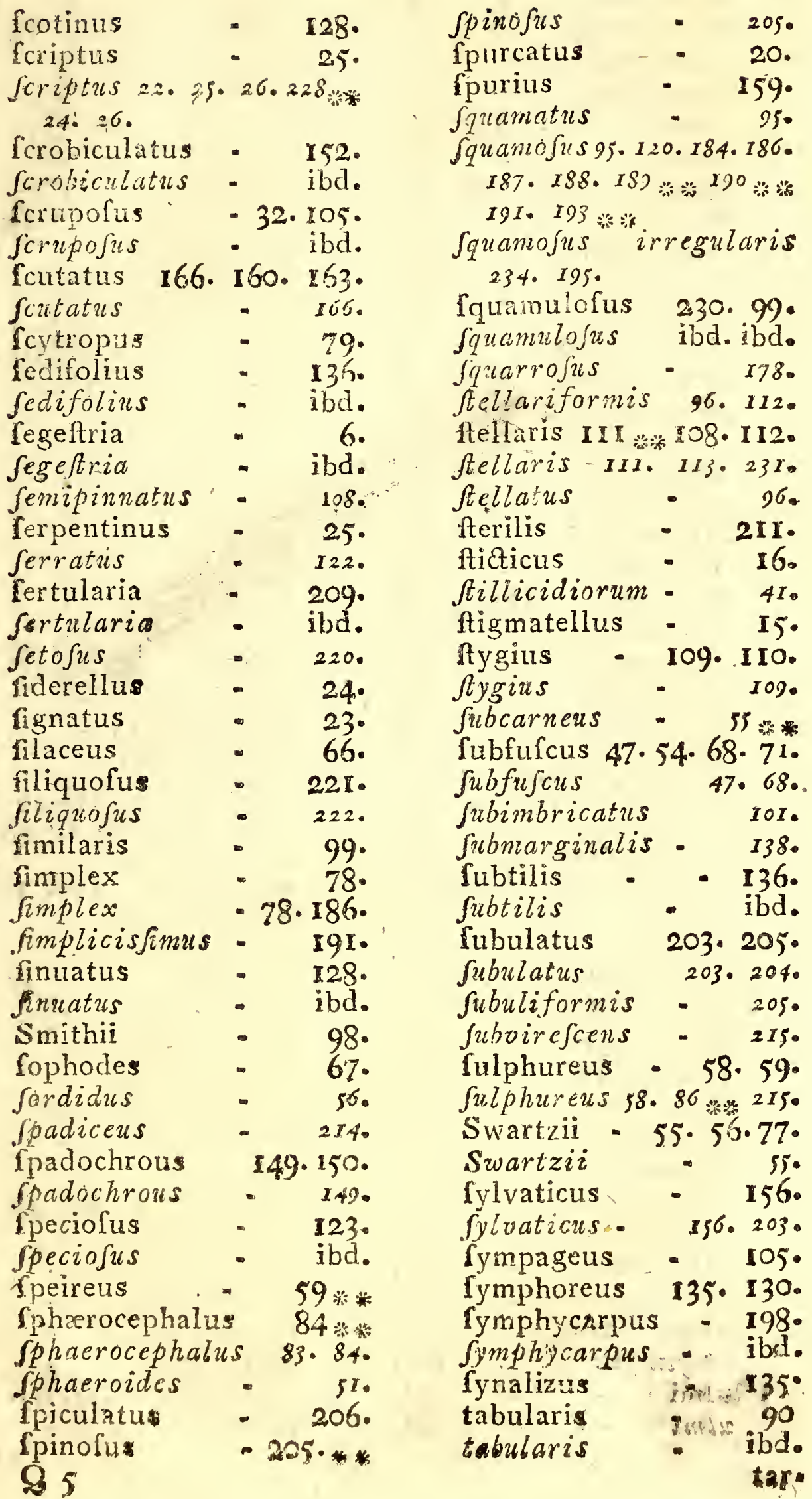




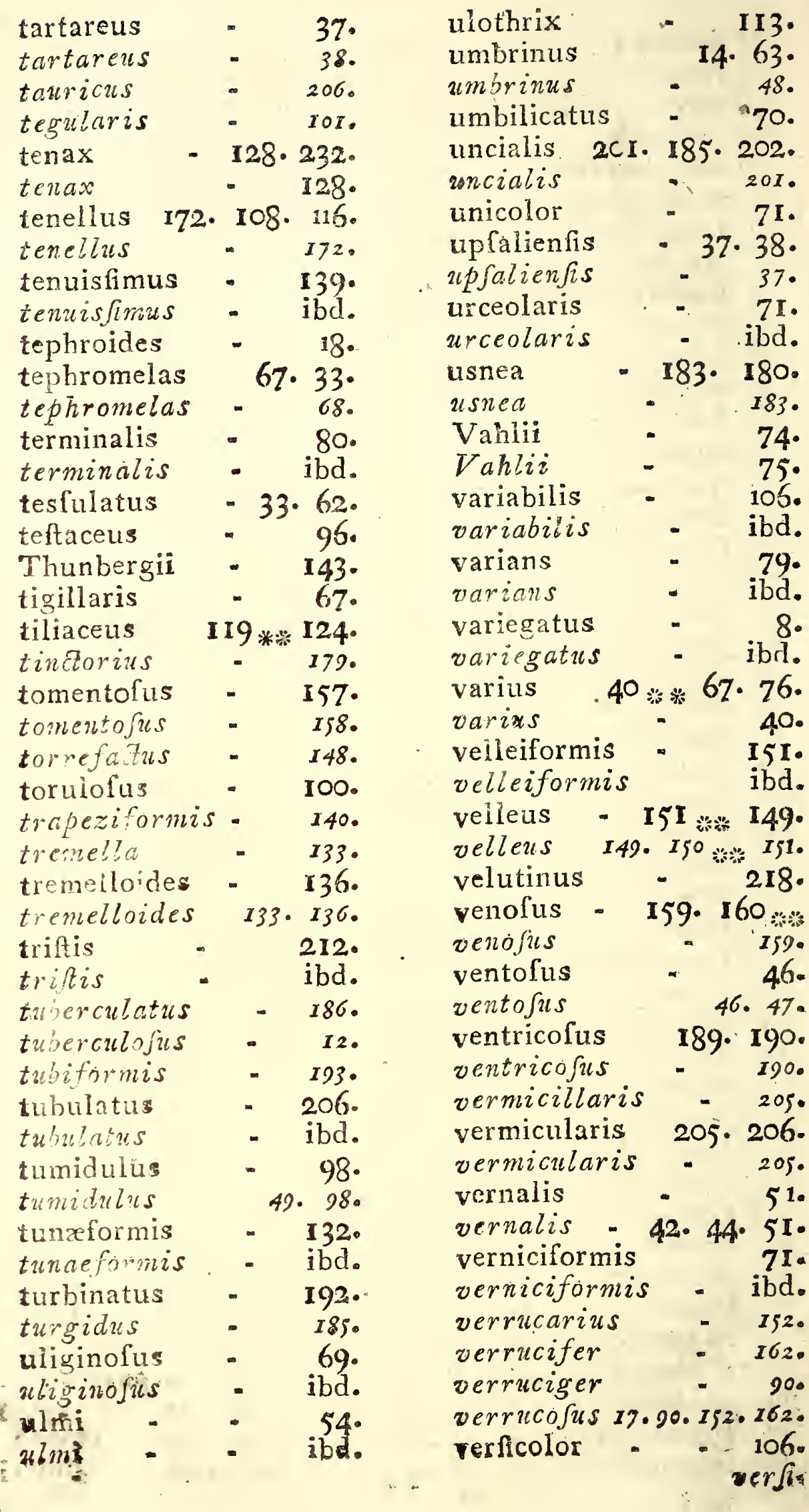




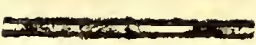

\begin{tabular}{|c|c|c|c|c|c|}
\hline verficolor & - & I06. & calycaris. & - & \\
\hline verticillatus & - & 222. & candelaris & - & $93 \cdot$ \\
\hline veficularis & - & 94. & caperata & - & 320. \\
\hline veficulofus & - & 137. & centrifuga & $=$ & 119. \\
\hline vespertilio & - & 1310 & ciliaris & - & 17 \\
\hline villofus & - & 218 & ciliata & - & $\boldsymbol{I}$ \\
\hline virellus & - & 108. & concolor & - & 9 \\
\hline vitellinus & - & $4 \mathrm{I}$. & crasfiformis & - & \\
\hline vitellinus. & - & ibd. & cucullata & - & \\
\hline viridatus & - & 53. & denudata & : & \\
\hline viridefcens & - & 50. & $d i f f u \int a$ & - & $\boldsymbol{I I}$ \\
\hline viridescens & - & 50.67. & dilacerata & - & \\
\hline viridiater & m & 79. & dubia & - & \\
\hline viridiater & - & ibd. & fahlunenfis & - & \\
\hline viridiflavesce & $n s$ & 71. & fallax & - & \\
\hline vividis & - & 10. 143 . & farinacea & - & \\
\hline viridulus & - & 2.9 & fraxinea & - & \\
\hline viridulus & - & ibd. & fuliginofa & - & 158 \\
\hline volvatus & - & 35. & fulva & - & \\
\hline vulgatus & - & $2 I$. & furfuras & - & 17 \\
\hline vulpinus & ; & 179. & glauca & - & 167 \\
\hline vulpinus & $\therefore$ & 180. & glomulifera & - & 15 \\
\hline vulvella & - & 22. & glomulifera & - & $i b c$ \\
\hline Weberi & - & I42. & groenlandica & - & 155 \\
\hline Weftringii & - & 88. & herbacea & - & 154 \\
\hline Wefringii & - & ibd. & herbacea & 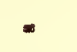 & ibd. \\
\hline Wulfenii & - & 34 . & $\begin{array}{l}\text { hispida } \\
\text { hottentotta }\end{array}$ & - & $\begin{array}{l}172 . \\
155^{\circ}\end{array}$ \\
\hline ICHENOIDE & s. & & incurva & $\infty$ & 107. \\
\hline ciliare & - & 173. & islandica & - & 15 \\
\hline flammeum & $a$ & 182. & juniperina & - & 168. \\
\hline furfuraceum & - & 174. & laqueata & - & 9 \\
\hline hispidum & - & 372. & miniato & - & 102. \\
\hline islandicum & m. & 170. & $\begin{array}{l}\text { muralis } \\
\text { nivalis }\end{array}$ & - & $\begin{array}{l}5040 \\
5>20\end{array}$ \\
\hline$O B A R Y A$ & 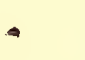 & 3. 152 . & olivacea & - & $x 22$ \\
\hline acetabulum & - & $\quad 122$ & oniphalodes & - & $\boldsymbol{I y}$ \\
\hline aculeata & - & 213. & orbicularis & - & II3 \\
\hline$a d u f t a$ & . & II5. & parietina & - & $\mathrm{I} 2 \mathrm{I}$ \\
\hline aipolia & - & 112. & perforata & - & 15 \\
\hline ambigua & - & 127 & perlata & - & 153 \\
\hline ampullacea & - & $155^{\circ}$ & perlata & - & ibd. \\
\hline anguftata & - & 213. & physodes & - & 116 \\
\hline aquaticat & - & 142. & pinaftri & - & 168 \\
\hline azalex & - & $155^{\circ}$ & polycarpa & - & 93 \\
\hline $\operatorname{cac} \sqrt{i} a$ & $\cdot$ & 108. & populina & - & $\begin{array}{l}\text { I } \\
\text { pro }\end{array}$ \\
\hline
\end{tabular}




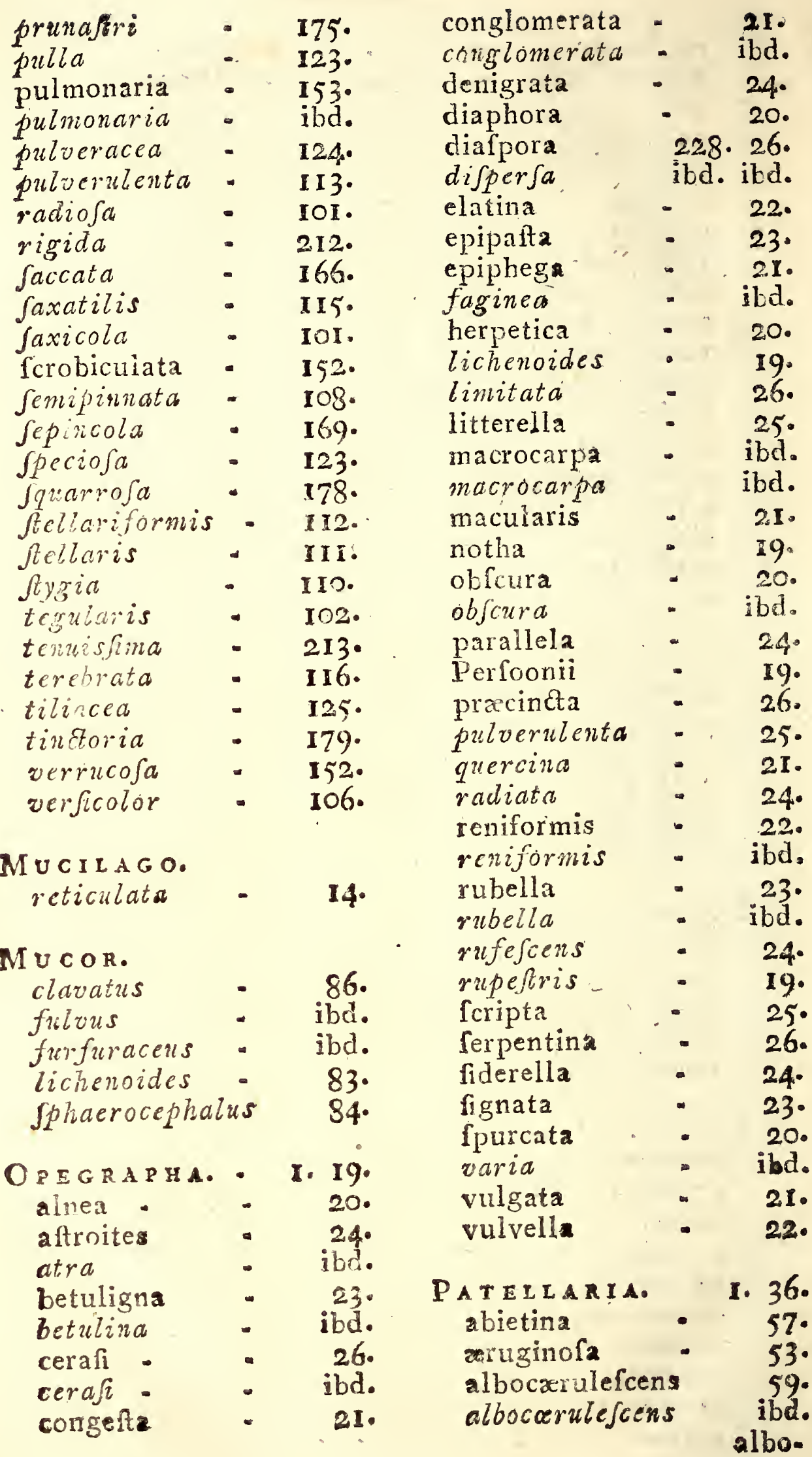




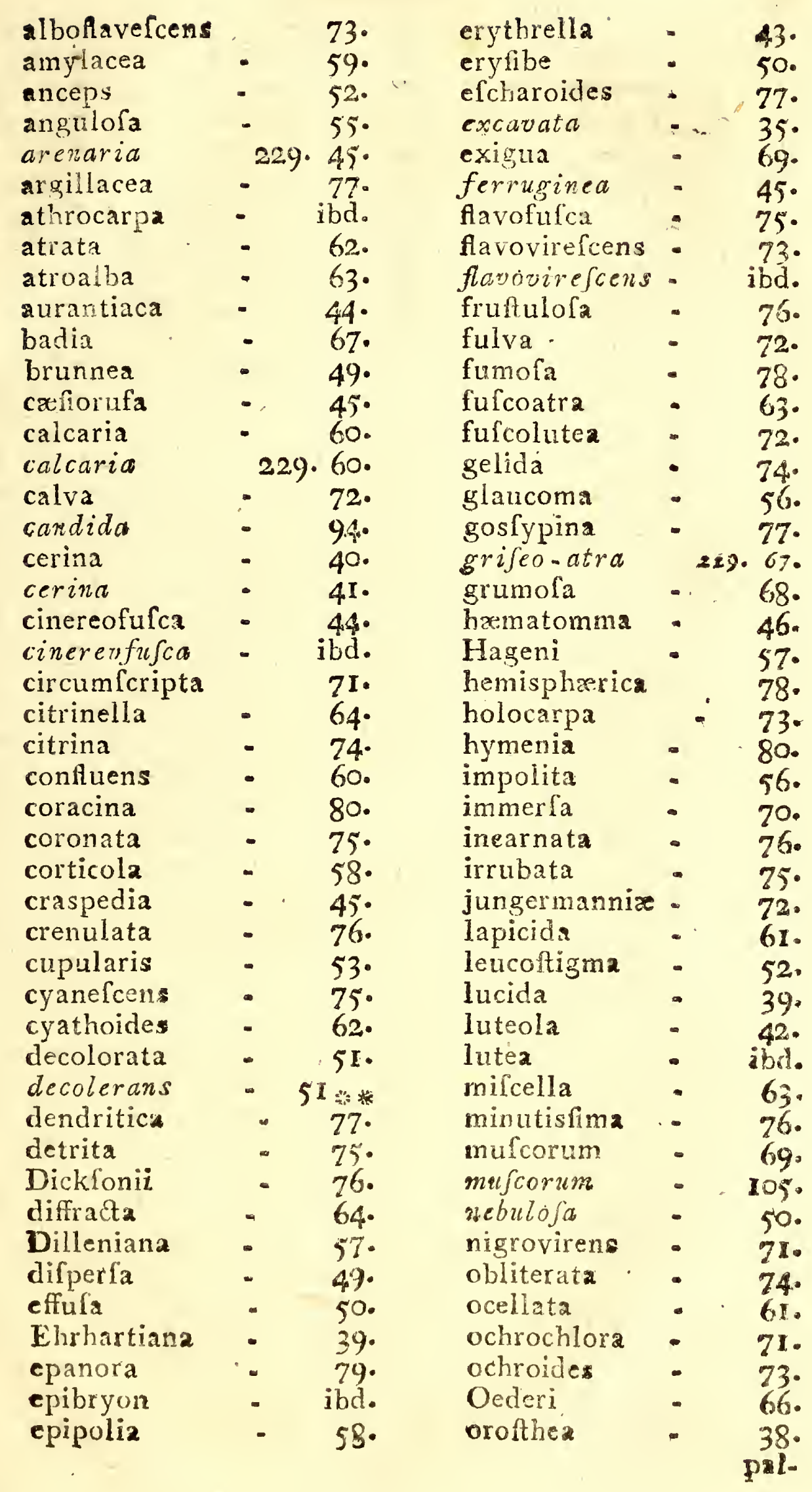




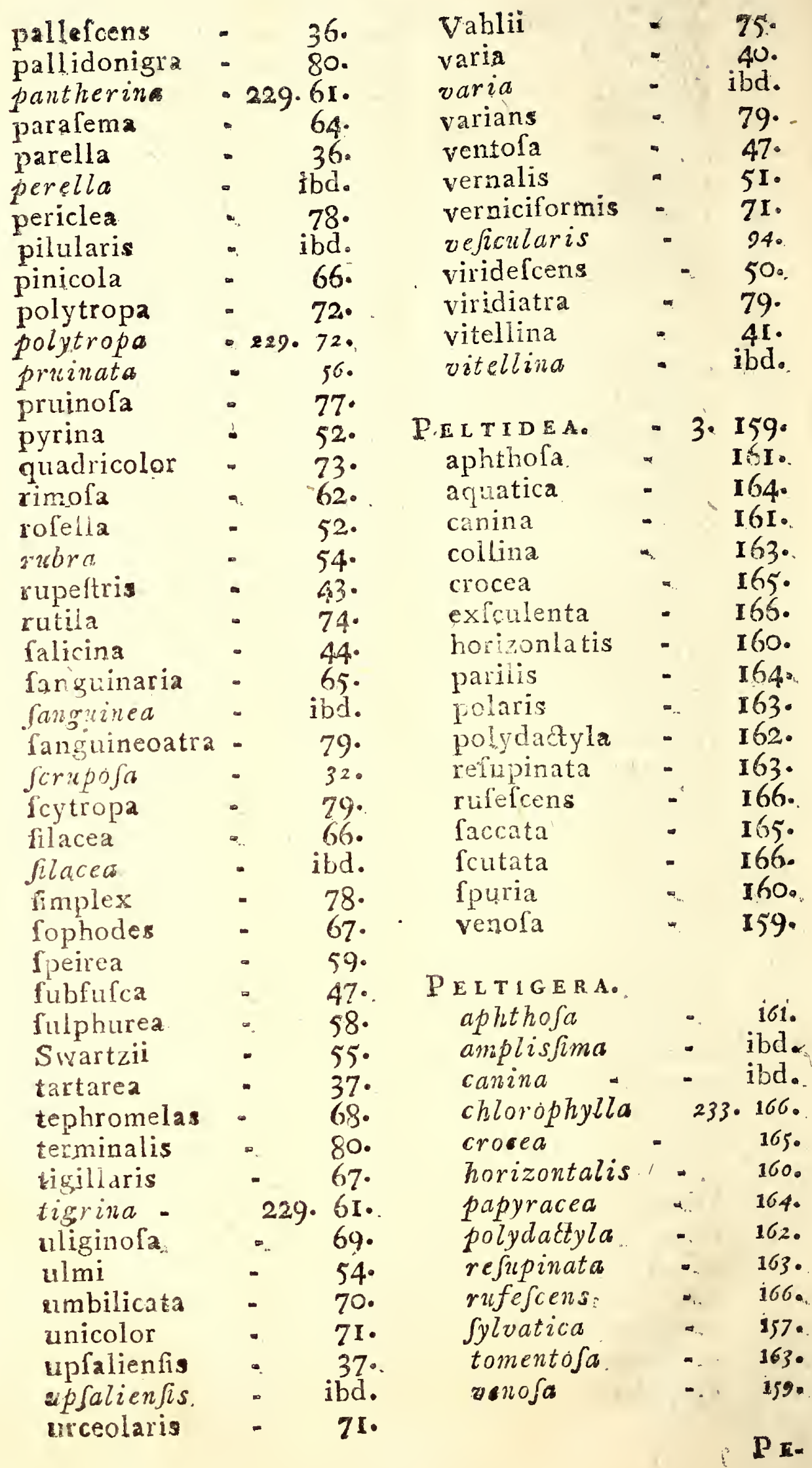


PIZA。

jenenfis

$54^{\circ}$

P IX Y S C 1 A. calicaris

capenfis

ciliaris

chryfophthalma

complanata

cucullata

farinacea

fafligiata

framea

Ravicans

fraxinea

fuciformis

furfuracea

islandia

leucomelos

linearis

nivalis

pollinaria

polymorpha

prunaftri

farmentofa

tenella

usnea

vulpina

P L A COD I M.

albefcens

bracteatum

candelarium.

canefcens.

circinatum

cocoës

demisfum

elegans

epigeum

fulgens

impresfum

lentigerum

murorum

pictum

faxicola

fympageum

\section{170. \\ 176. \\ 182. \\ 173. \\ - $18 \mathrm{I}$. \\ - I82. \\ - $\quad 17 \mathrm{r}$. \\ - $\quad 177$. \\ - $\quad 176$. \\ - 182. \\ - 183. \\ - $\quad 175$. \\ - $\quad 182$. \\ - $\quad 170$. \\ - I8I. \\ - ibd. \\ 179. \\ 174 . \\ 180. \\ 172. \\ $183^{\circ}$ \\ I79.}

- $\quad 173$.

- 171 .

- 178.

2. 100 .

105.

103.

93.

103.

100.

106.

104.

102.

$105^{\circ}$

.

- 105.

105.

IOI.

106.

104. verficolor $=105$.

variabile $*$ ibd.

PLATISMA * 3. 167.

amprellaceum - $\$ 55^{\circ}$

aquaticum - 142.

armotrum - 181.

caperatim - 120.

cornudamae - 157.

crocatum - 158.

denudatum - 181 .

fallax " 170.

faliax - 169.

filix 232. 159 .

fraxineum - $175^{\circ}$.

glaucum - 167.

juniperinum - 168 .

perforatum - $155^{\circ}$

pinaftri - I68.

frepincola - $\quad 169$.

Sepincola - ibd.

Ps ORA.

alabaftrina $\quad$ - $360^{\circ}$

albescens - 105.

bracteata - 103.

brunnea - . 49.

caelata - - 98.

caefia - 108.

candida - - 94.

canefcens - IO3.

carnasa $\quad 91$.

citrina - 102.

coronata $\quad 230.75$.

deaurata $\quad 94$.

decipiens - 96.

dendritica - 77.

epigeja - 105 .

frigida - 38.

gelidiformis - 99.

grifea - 91.

gypsophila - 98.

hypnorum - 94.

lactea - 28.

lentigera $: 103$.

mierophylla - 91. 
miniato

muralis

muscorums

- freata

paradoxa

jezizodes

radiofa

faxicola

fimilaris

Squamota

teftacea

turbinata

upsalienfis

variabilis

vefacularis

P.

crelata

candelaria

cartilaginea

chryfoleuca

crasfa

decipiens

selidiformis

sypjophila

hypnorum

leucophrea

Jurida

microphylla

nigra

paradoxa

rubina

rubiginofa

raxitiraga

fcalaris

fimilaris

Smithii

fquamulofa

teftacea

torulofa

tumictula

veficularis

PULMONAIA.

herbacea

reticulata

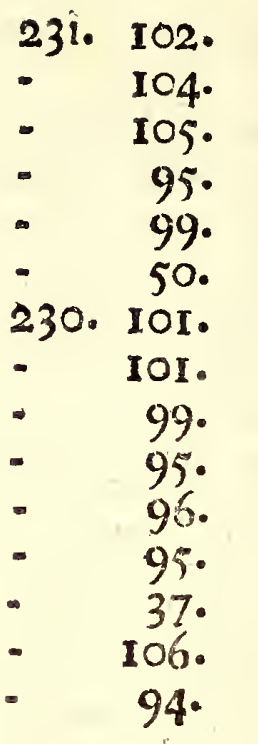

2. 91.

- 98.

- $\quad 92$.

- 97.

- 99.

- 97.

- 96.

- 99.

- 98.

- 93.

- 99.

- 95.

- $9 \mathrm{I}$.

- 92.

- 99.

- 100.

- 99.

- 100.

- 95.

- 99.

- 98.

230.99.

- 96.

- 100.

- 98.

- $94^{\circ}$

RHIZOMOR PHA.

$\begin{array}{lll}\text { capillaris } & - & 222 . \\ \text { cinchonce } & - & \text { ibd. } \\ \text { fragilis } & - & \text { ibd. }\end{array}$

SCYPHOPHORUS. 3. 183 . alcicornis - I84. bellidiforus - 194. ceratophyllus $\quad 196$. cervicornis - 184. cocciferus - 188. cornutus - I92. deformis - 189. digitatus - 188. elongatu - 1960. endivifolius - ibd. epiphyllus - $\mathbf{1 8 5}$. fibula - I94. filiformis . 193. fimbriatus , - $187^{\circ}$ gracilis - I9r. monocarpus - 196. parechus - $185^{\circ}$ pleolepis - 195. pyxidatus = 196. radiatus - $\quad$ r90. turbinatus - I92. ventricofus - I90.

SETARIA. 4. 2 I9. chálybeiformis 220. dichotoma - $22 \mathrm{I}$. filaris - ibd. hippotrichodes 220. juiata - . 219. roceella - 221. filiquofa - 222.

SPHARIA. 58.
levcocephala lichenoides - 64. nitide .

- 18. 


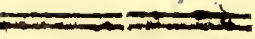

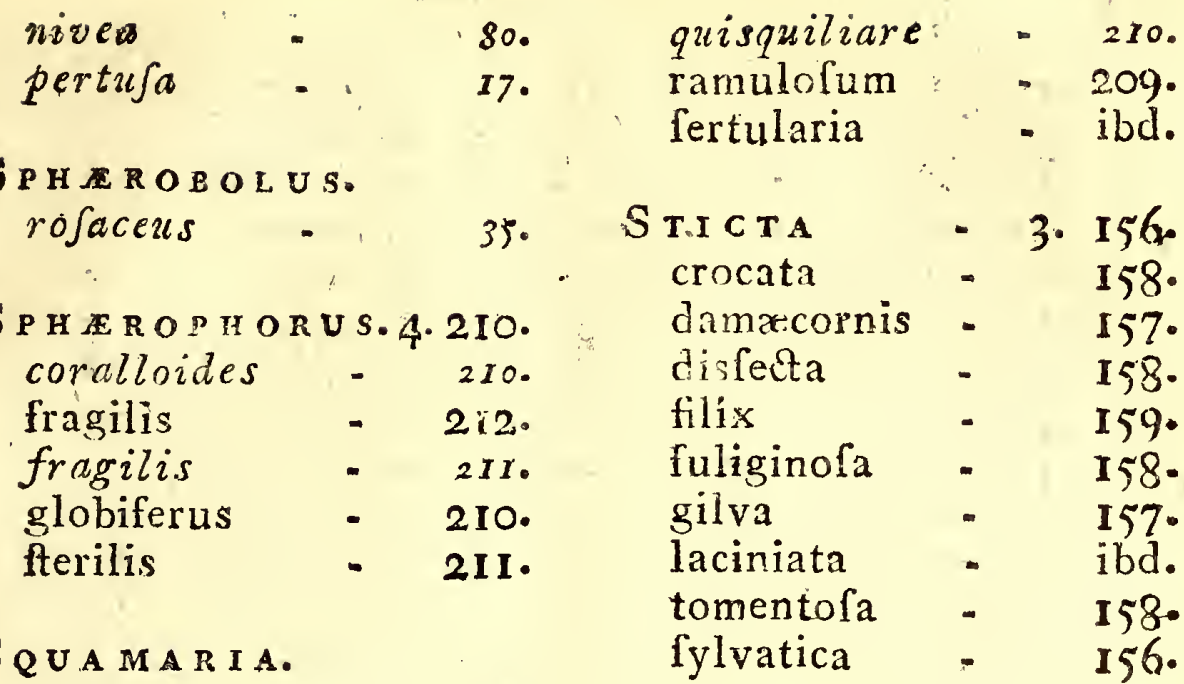

$\begin{array}{lll}\text { ambigua } & - & 117 . \\ \text { centrifuga } & - & 118 . \\ \text { fahlunenfis } & - & 110 . \\ \text { juniperina } & - & 168 . \\ \text { Pinalri } & - & \text { ibd. } \\ \text { pulla } & - & 123 . \\ \text { Dulverulenta } & - & 113 . \\ \text { rubina } & - & 100 . \\ \text { Jygia } & - & 110 .\end{array}$

STEMONITIS.

$\begin{array}{lrr}\text { fulva } & - & 86 . \\ \text { furfuracea } & - & \text { ibd. } \\ \text { lichenoides } & - & 84 . \\ \text { nivea } & - & 85 . \\ \text { Sthaerocephala } & 84 .\end{array}$

STEREOCAULON. $4 \cdot 208$. condenfatum - 209 . condenfatum - ibd. confine - - ibd. confine - . ibd. corallina - 87. corallinoides $9 \pi .92$. corallinum - 87. fragile $\quad . \cdot 2 \pi r$. globiferum $\quad 210$. madreporiforme 89. obtufatum - 20\%. pafchale , 208. paschale - ibd. quisquiliare " 210.
TH正工 PHORA. carnea

ISI.

TREMELLA. botryoides - 10. lichenoides - 133.

TRICHIA. $\begin{array}{llr}\text { flana } & - & 85 . \\ \text { fulva } & - & 86 . \\ \text { furfuracea } & \text { ibd. } \\ \text { lenticularis } & - & 84 . \\ \text { nivea } & - & 85 . \\ \text { Sphaerocephala } & - & 84 .\end{array}$

TUBERCULARIA. $\begin{array}{ll}\text { ericetorum } & \quad 81 . \\ \text { fungiformis } & \quad 82 . \\ \text { rofea } & 227.9 .\end{array}$

U M B I I I C A R I A. 2. I44. anthracina $\quad 144$. cirrosa $=149$. corrugata - 147. crinita - 149 . eylindrica - ibd. deufta $\quad 145$. erofa - IAs. ernfa - ibd. exafperata - 147. flocculosa : $14 i^{\circ}$ gla- 


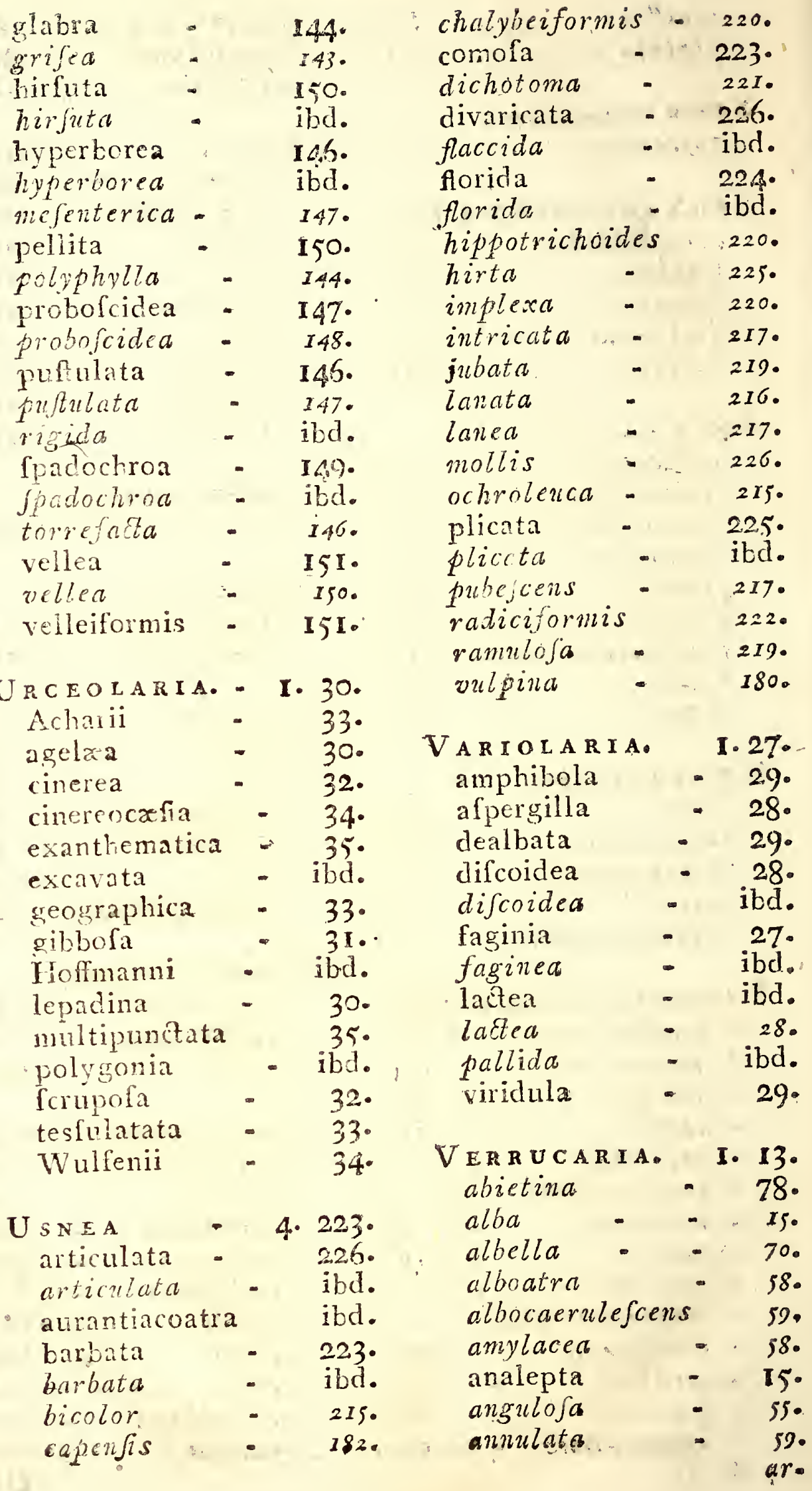




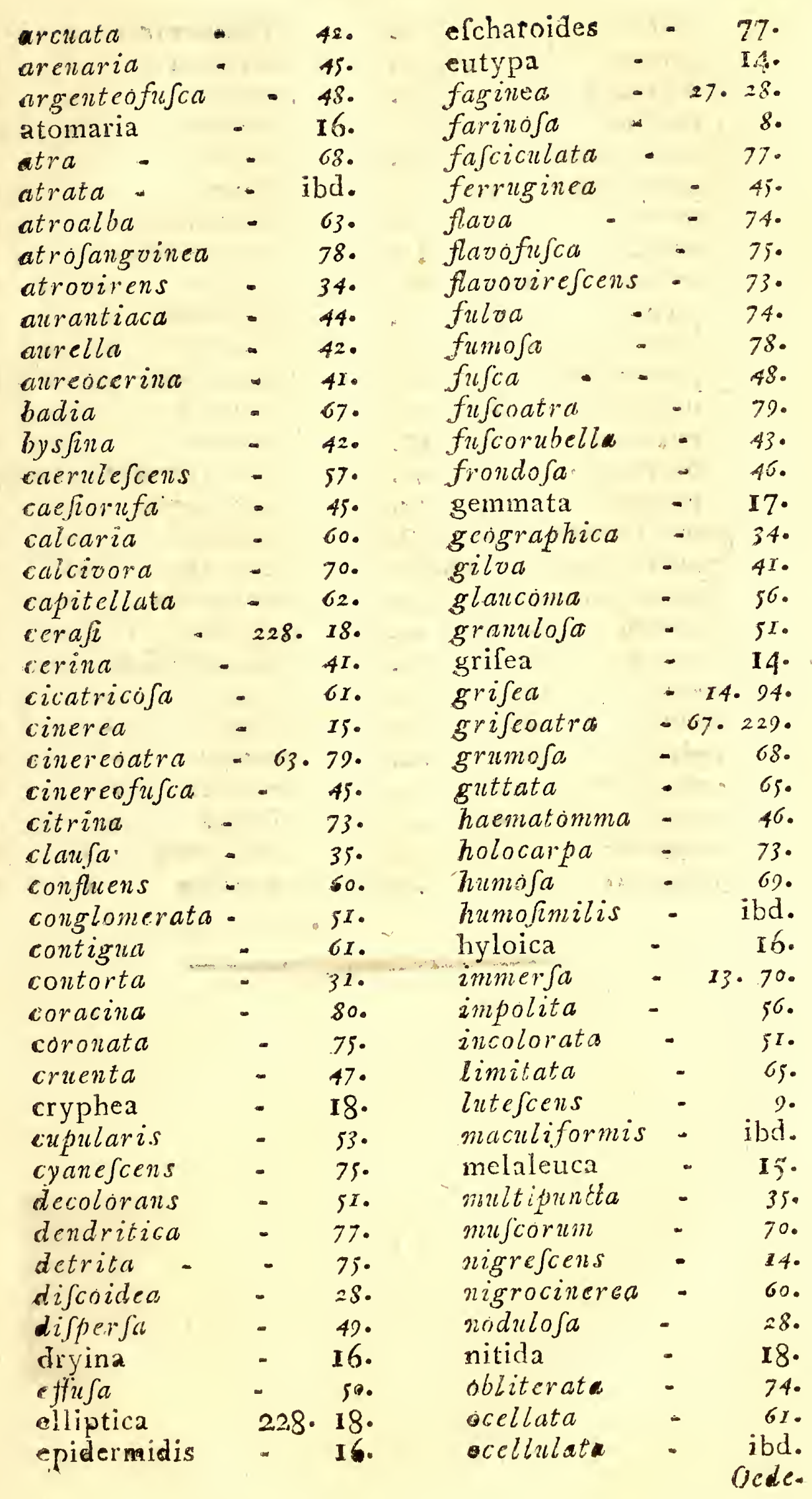




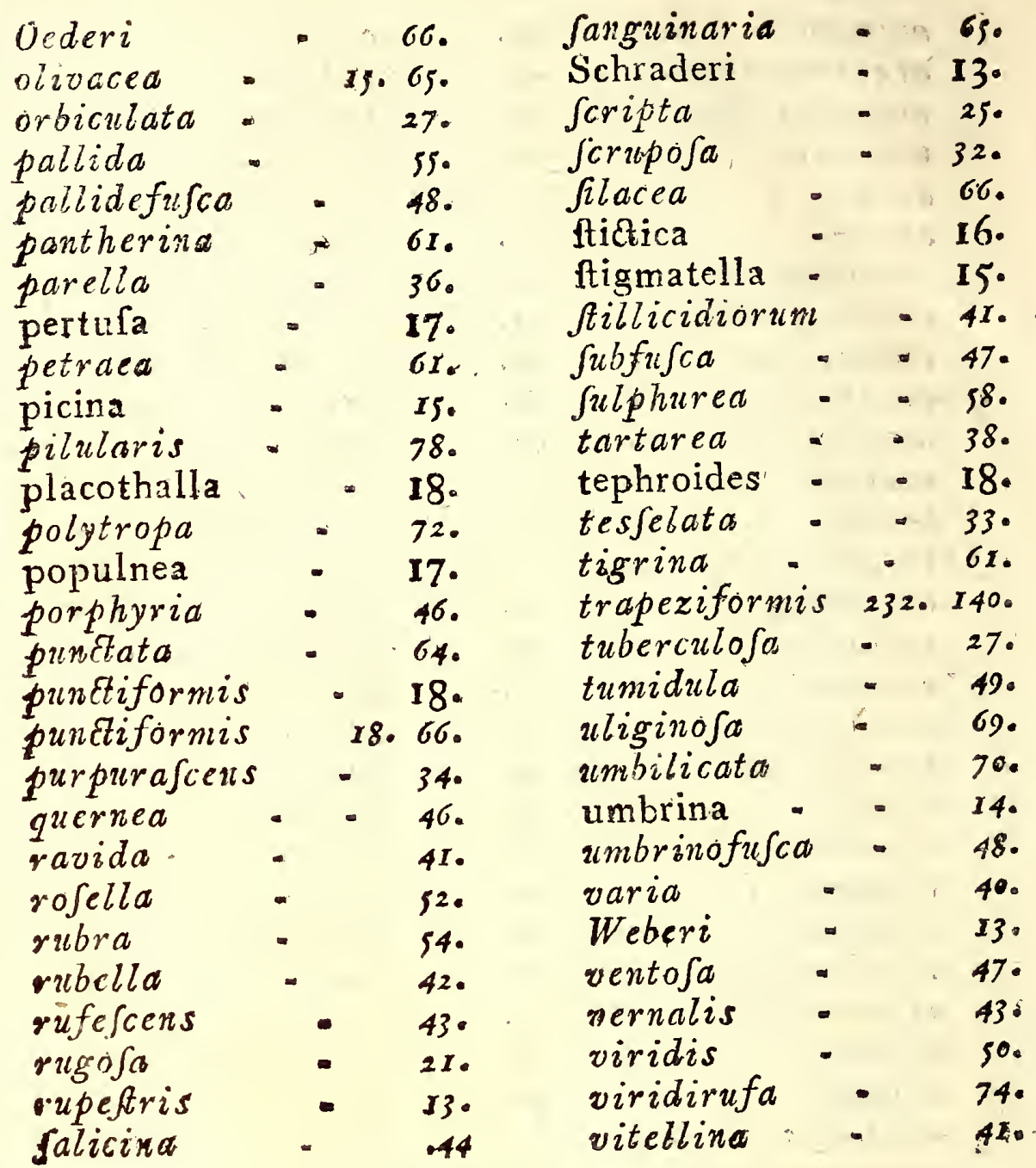


INDEX SVECICUS.

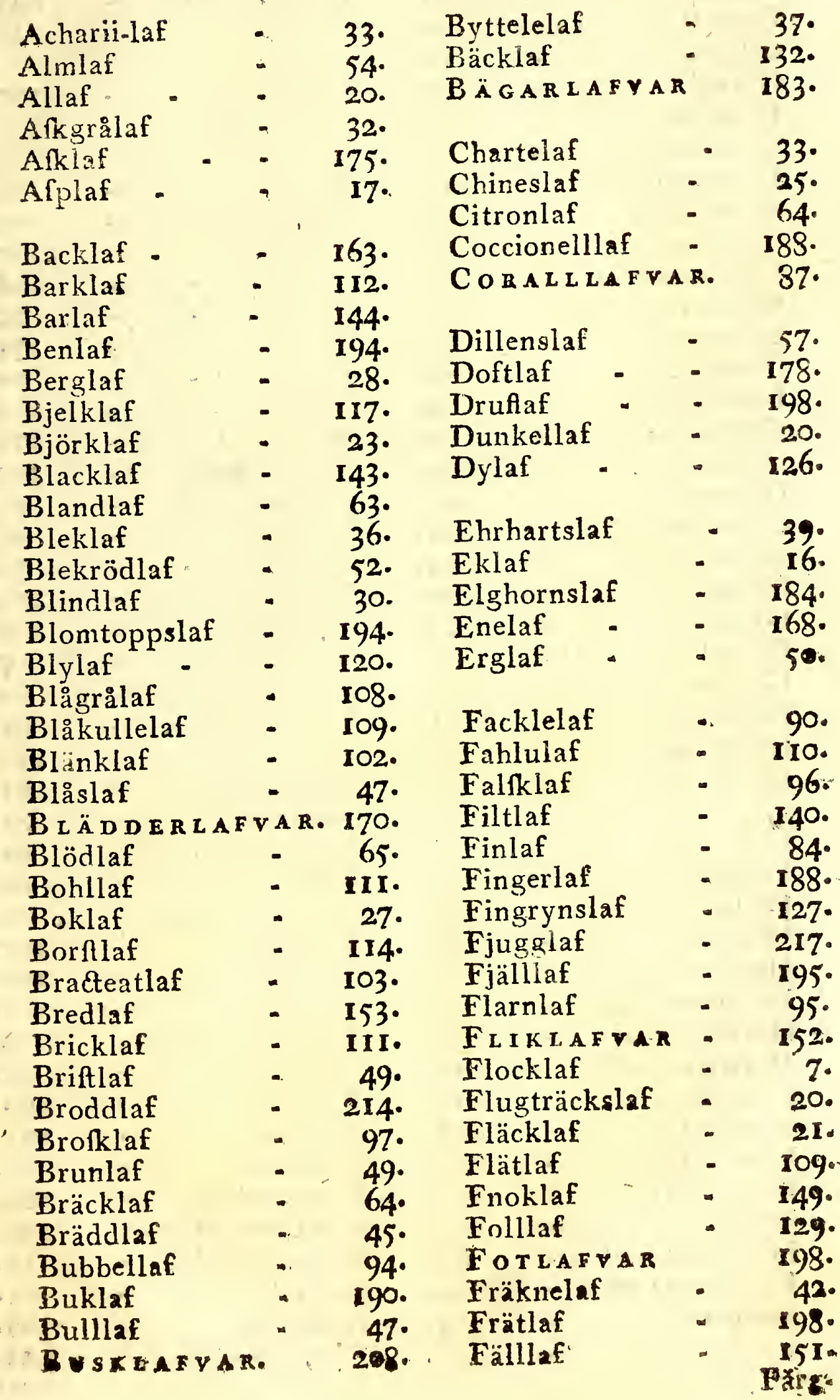




\begin{tabular}{|c|c|c|c|}
\hline Färglaf & $115{ }^{\circ}$ & $\begin{array}{l}\text { Hallaf } \\
\text { Harkantslaf }\end{array}$ & $\begin{array}{r}=\quad 70 . \\
=\quad 173 .\end{array}$ \\
\hline Gaffellaf & 204. & $\mathrm{H} \AA \mathrm{RLAF} V \mathrm{AR}$ & 223. \\
\hline & II 9. & Hällelaf & $38 \cdot$ \\
\hline $\begin{array}{l}\text { Gipslaf } \\
\text { Gjällaf }\end{array}$ & 59. & Isgralaf - & \\
\hline Glänslaf & $6 ?$ & Island’siaf & $=\quad 170$. \\
\hline Gniflaf & $15 \cdot$ & & \\
\hline Granbarkslaf & $57 \cdot$ & Kaklaf & II8. \\
\hline $\mathrm{G}_{\mathrm{r}}$ & 16. & Kaikiaf & 60. \\
\hline Granrotslaf & 188 & Kammlaf & $\therefore \quad 127$. \\
\hline Groddlaf & 60. & Kantiaf & ibd. \\
\hline GROPLAFVAR & 30. & Tardlaf & $84^{\circ}$ \\
\hline Gruslaf & 39. & Kafklaf & $\mathbf{I 7} \mathrm{I}$ \\
\hline Grynlaf & $55^{\circ}$ & Klaslaf & $2 I$. \\
\hline Gro & 68 . & Kleniaf & 216. \\
\hline Grabrunlaf & 44. & Klilaf & 117. \\
\hline Grågrönlaf & Io\%. & KLIBBLAFVA & $1 \div 5$ \\
\hline Gro & 14. & Klippingslaf & 160. \\
\hline dlaf & $45^{\circ}$ & Klipplaf & 56. \\
\hline $\mathrm{Gr}$ & 64. & af & 86. \\
\hline rönlaf & 154. & lubblaf & 176. \\
\hline Gri & 10. & KLit & 210. \\
\hline inlaf & 42. & Klyftlaf & $7 \cdot$ \\
\hline G & 6. & Knaggellaf & 89. \\
\hline $\mathrm{Gu}$ & II. & KNAPREAEV & 83. \\
\hline Gy & $43^{\circ}$ & af & 130. \\
\hline haf & $2 \mathrm{I} \cdot$ & Knotterlaf & $5^{\mathrm{I}}$ \\
\hline ellaf & I69. & $\begin{array}{l}\text { Knölbladslaf } \\
\text { KNóLA Fvar. }\end{array}$ & $\begin{array}{l}185^{\circ} \\
8 \mathrm{I}^{\circ}\end{array}$ \\
\hline slaf & 57. & Kollaf & 62. \\
\hline edwirslaf & $8 \mathrm{I} \cdot$ & KOPPLAFVAR. & $27 \cdot$ \\
\hline $\begin{array}{l}\text { edwigslaf } \\
\text { innlaf }\end{array}$ & 140. & Kornlaf & $-\quad 125^{\circ}$ \\
\hline inlaf & 121. & Kra & $=100$. \\
\hline $\begin{array}{l}\text { jorthornslaf } \\
\text { Ioffmanslaf }\end{array}$ & $184^{\circ}$ & It & 108 \\
\hline $\begin{array}{l}\text { offmanslaf } \\
\text { ornbladslaf }\end{array}$ & $3 \mathrm{I}$. & KRETSLAFVAI & 100. \\
\hline $\begin{array}{l}\text { rnbladslat } \\
\text { R N LAFVA }\end{array}$ & $185^{\circ}$ & $\begin{array}{l}\text { Kritlat } \\
\text { Krittellaf }\end{array}$ & 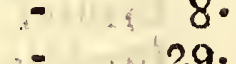 \\
\hline $\begin{array}{l}\text { OR L LAFVA } \\
\text { udlaf }\end{array}$ & 212. & $\begin{array}{l}\text { Nrittentar } \\
\text { Kronlaf }\end{array}$ & $\begin{array}{r}=29 . \\
=\quad 126 .\end{array}$ \\
\hline udla & $\begin{array}{l}119 . \\
161 .\end{array}$ & Krumlaf & $\begin{array}{l}=126 . \\
\therefore \quad 107 .\end{array}$ \\
\hline $\begin{array}{l}\text { lundlat } \\
\text { lvitblalaf }\end{array}$ & $\begin{array}{l}161 \\
59 .\end{array}$ & Kruskantlaf & $68^{\circ}$ \\
\hline vitgrålaf & $=103$. & KRUSLAFVAR. & 167 \\
\hline Hitlaf. & $-7 \cdot$ & Kräftftenslaf & - \\
\hline Hvitprickslaf & - 152 & Kuplaf & 32. \\
\hline $\begin{array}{l}\text { YLSL AFVAR. } \\
\text { alk }\end{array}$ & 210 & Kuttiaf & 209. \\
\hline & & & Le \\
\hline
\end{tabular}




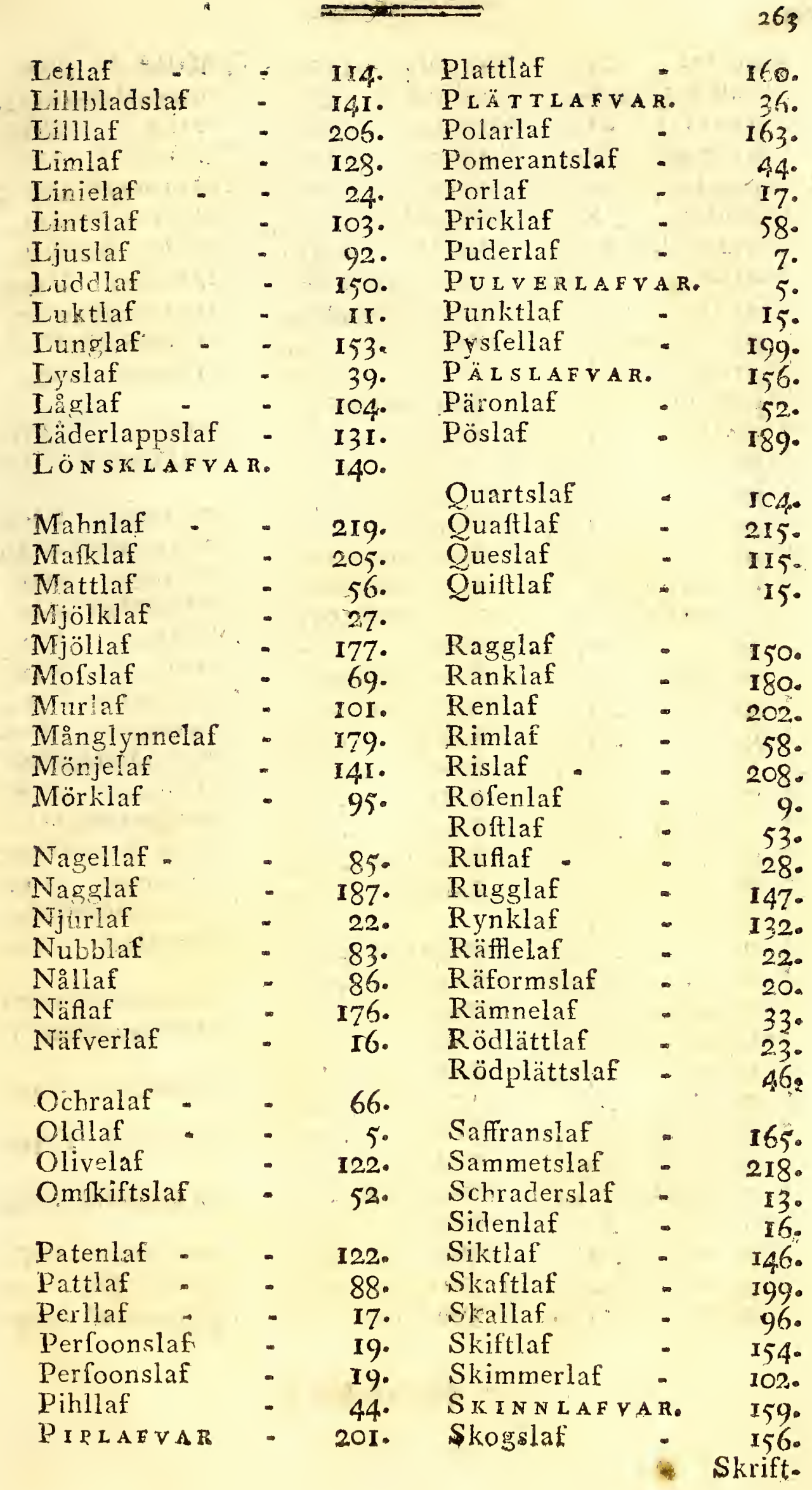




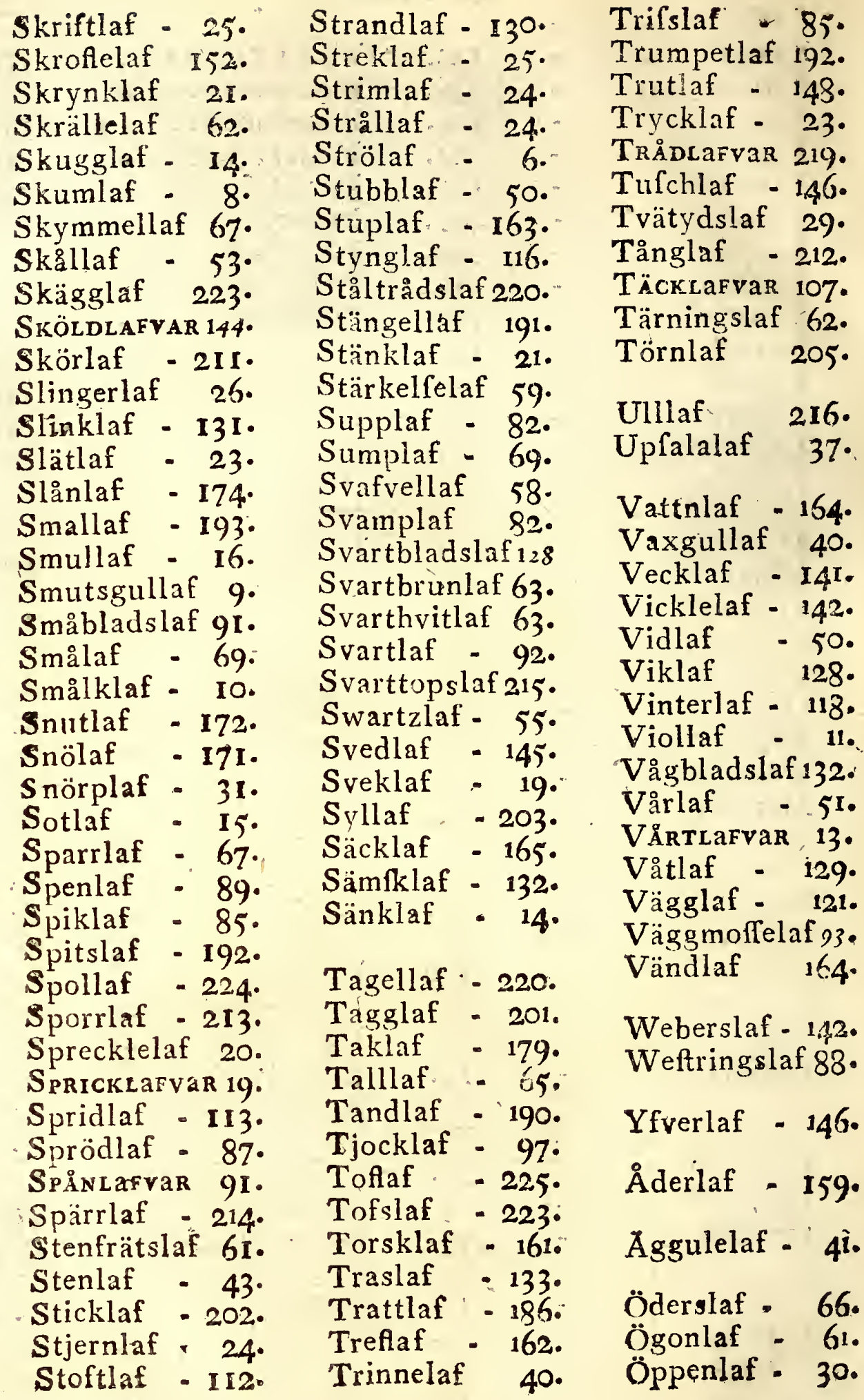


Fig. 1.

Tab.1
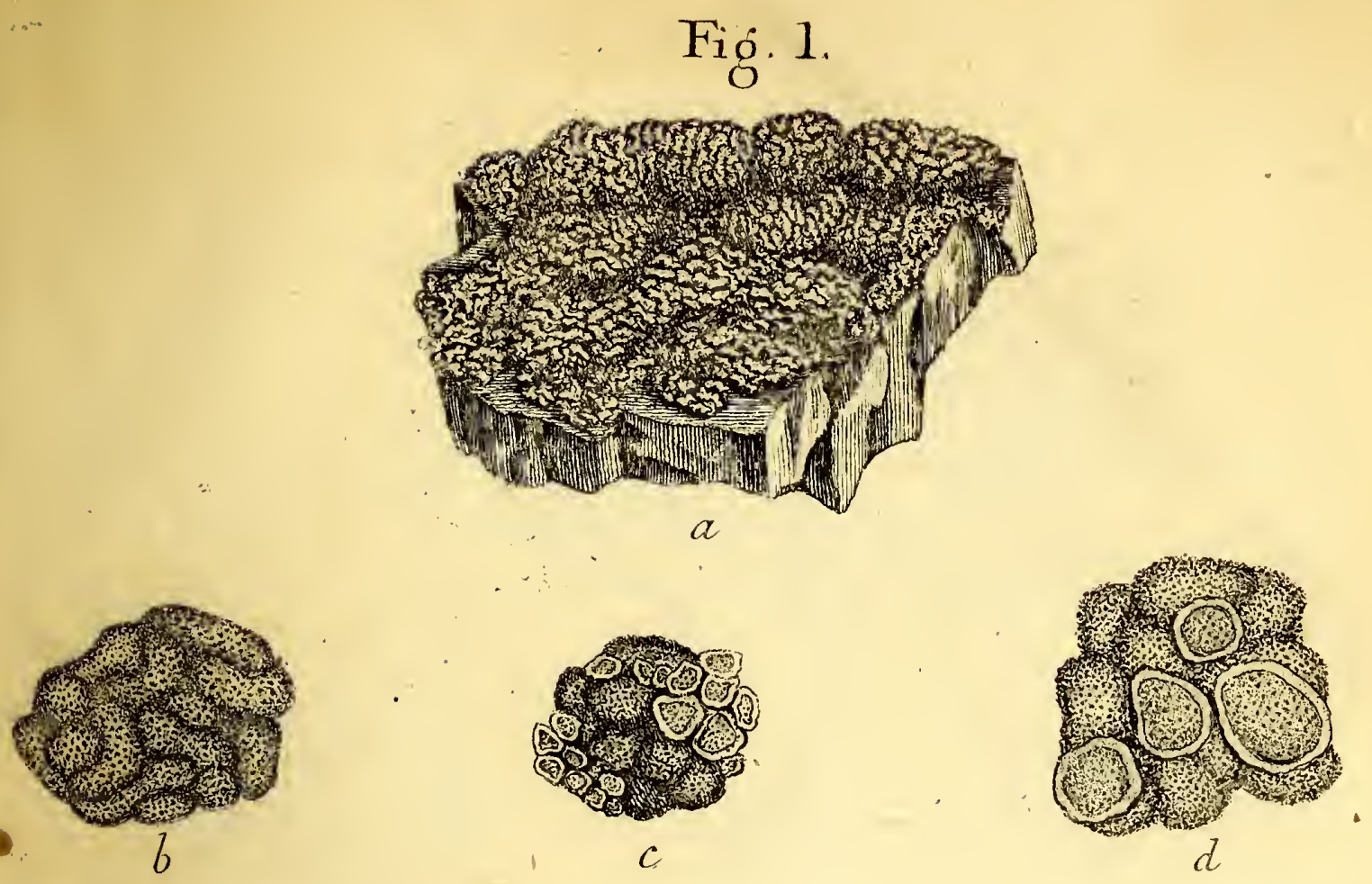

- Sichon Dillenianus.

Fig. 2 .
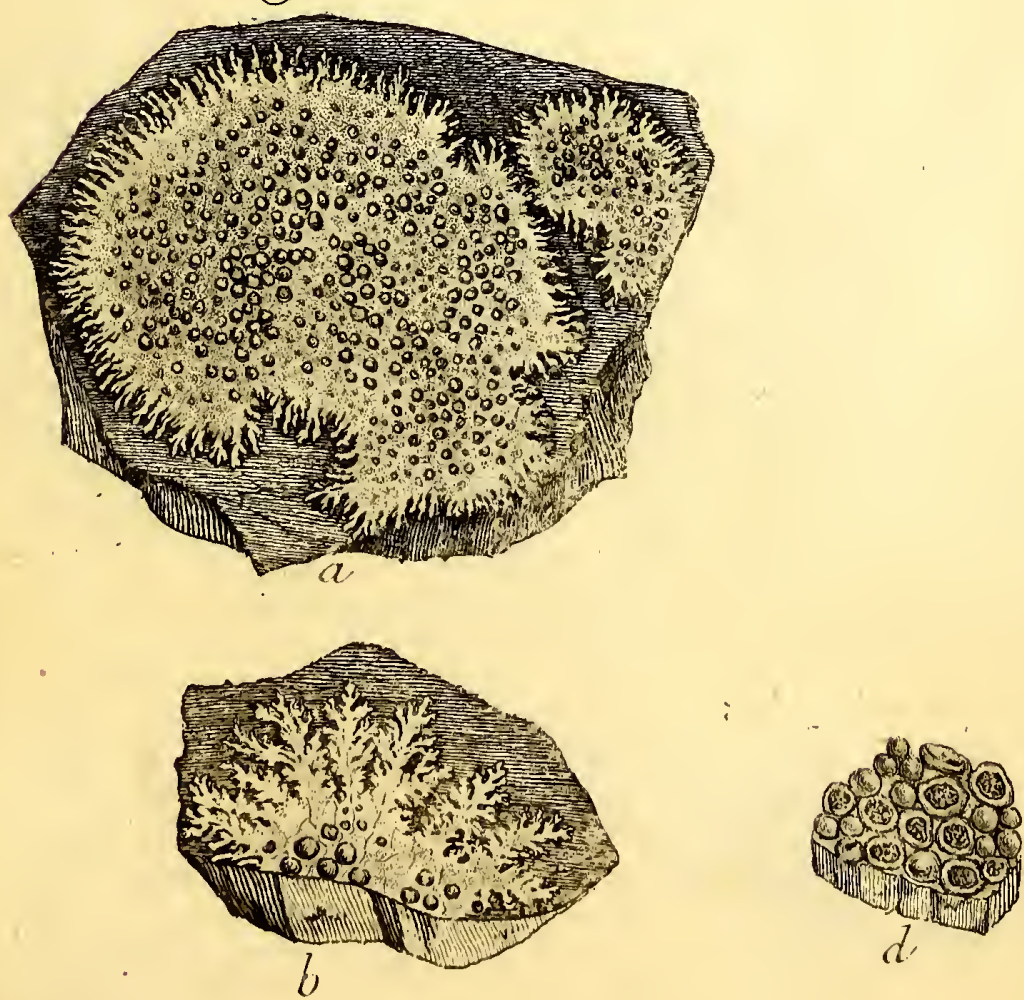

Lichen (1) Promitie. 



ho

$$
22079
$$





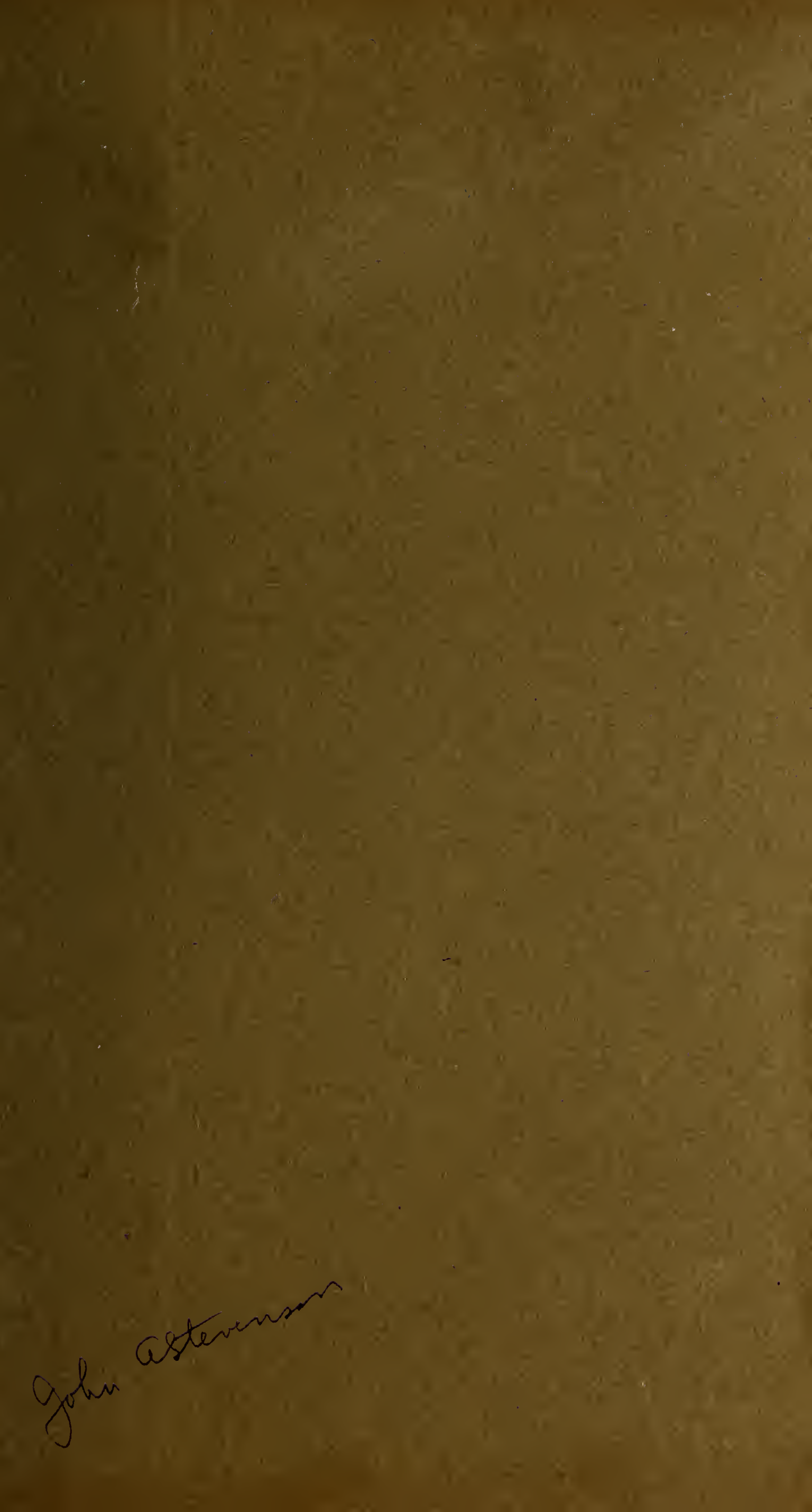




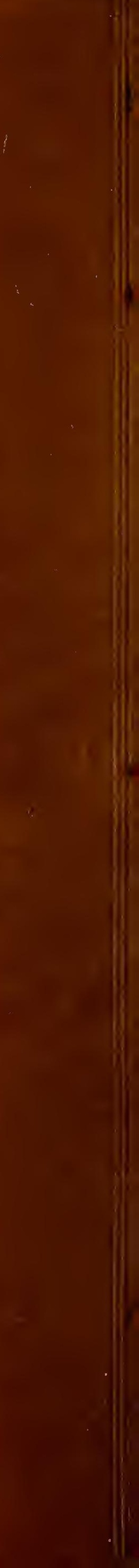

